\title{
Investigating the effects of novel kappa opioid receptor agonists on the dopamine transporter
}

BRIDGET SIMONSON

A thesis

submitted to the Victoria University of Wellington in fulfilment of the requirements for the degree of Doctor of Philosophy 



\begin{abstract}
Classic kappa opioid receptor (KOPr) agonists have shown anti-addictive properties in rat models of addiction (Heidbreder et al. 1998; Schenk et al. 1999; Sun et al. 2010), and this has been shown to be partially through modulation of dopamine and serotonin in the synapse (Thompson et al. 2000; Zhang et al. 2004; Zakharova et al. 2008a). However, they have side effects such as depression and dysphoria and therefore have not been moved into the clinic. The novel KOPr agonist salvinorin A has a completely different structure compared to the classic agonists, and along with its novel analogues has opened up a new family of KOPr agonists which may possess anti-addictive properties and have the potential to have decreased side effects. Salvinorin A has also demonstrated anti-addictive properties (Morani et al. 2009). In this study the novel KOPr agonist salvinorin A and its analogues DS-1-240 and DS-3-216 were investigated, along with the classic agonists U50,488H and U69,593. Their effects on the dopamine transporter (DAT) were measured using isolated rat brain tissue and cell models. The effects of U50,488H and salvinorin A on the serotonin transporter (SERT) was also measured in rat striatum using rotating disk electrode voltammetry, which was established to measure serotonin uptake in our lab during this study.
\end{abstract}

We found that all of the kappa opioid receptor agonists studied in isolated rat brain tissue caused dose dependent increases in uptake of dopamine by the dopamine transporter and a decrease in uptake of serotonin by the serotonin transporter. The effect on the serotonin transporter was observed after a 15 min incubation with the agonists. Salvinorin A had a faster effect on the dopamine transporter than the other compounds investigated, with increases measured at $1 \mathrm{~min}$ rather than $4 \mathrm{~min}$. DAT kinetics showed increases in Vmax for all agonists investigated, and both U69,593 and DS-1-240 also showed increased Km values. This demonstrates an overall increase in function, with the possibility of increased cell surface expression.

Further investigation using cell models also found an increase in uptake of the fluorescent monoamine transporter substrate $\mathrm{ASP}^{+}$by YFP tagged human DAT (YFPhDAT). This effect was seen with all the agonists studied after incubations of less than 5 min and was YFP-hDAT trafficking-independent. The increase in uptake seen may be due to increased active YFP-hDAT found on the cell membrane as $\mathrm{ASP}^{+}$binding studies demonstrated an increase in binding. The acute increase in YFP-hDAT function 
was found to be ERK1/2 dependent for all compounds studied, and was also dependent on intact lipid rafts in the cell membrane. After a 30 min incubation, salvinorin A and U50,488H still caused increased uptake of $\mathrm{ASP}^{+}$by YFP-hDAT, whereas DS-1-240 and DS-3-216 did not. Increases in cell surface expression of YFP-hDATwas seen at this time point with salvinorin A, U69,593, and DS-3-216. Further investigation into this found that the increase in cell surface expression of YFP-hDAT after salvinorin A treatment was ERK1/2 dependent, whereas the increase seen with U69,593 appeared to be ERK1/2 independent.

Overall, this data demonstrates that KOPr rapidly regulates DAT function by a trafficking-independent, ERK1/2-, and lipid raft-dependent mechanism. The classic KOPr agonist U50,488H and salvinorin A also caused a decrease in serotonin uptake by SERT, confirming that the KOPr also regulates SERT. The data from this study provides more information on how these classic and novel KOPr agonists function to regulate DAT and SERT, which may help explain some of the anti-addictive properties displayed by these compounds. 


\section{Acknowledgements}

This has been a crazy undertaking, and I would not have made it through alive or still relatively sane without the following people:

My supervisors Bronwyn Kivell and John Miller, thank you for all your support and ideas (and chocolate, wine, and Christmas cookies)!

My fellow lab mates, especially Kirsty Danielson who has gone through this as well and has provided; a shoulder to cry on, many research ideas, and a bottle of wine when needed, Aashish Morani, Peter Bosch, Ana Holley, Miles Benton, and our volunteers/minions/monkeys who then became our honours/masters students: Pam, Mark, Fraser, and Xiao-Wen for the large amounts of dishes, rubbish, and Western blotting you helped with. You have all provided endless fun and laughter, thank you!

The peeps who left us to move to greener grass (the Alan McDiarmid building), especially Ariane Chan, David O'Sullivan and Ryan Steel.

The technicians; Sushila Pillai for her help with confocal, Lee Botes for being the best Olympus rep ever, Richard Moore for his rats and helping me overcome (slightly) my fear of rodents, and Craig Doney, Neville Higgison, and Cameron Jack for helping our lab equipment to run smoothly, especially helping with RDEV.

My family who put up with me sharing my rants and successes, even though they had no idea about what I was talking about. Thank you for your never-ending love and support.

My friends who kept me slightly normal through this, especially Rach, Sarah, Jen, and Alex.

And of course God, who provided strength for me to keep going when things were tough, and to my church families in Wellington and Wanganui who have been praying since day one.

Funding for this project was provided by: WMRF, HRC, Neurological Foundation, and Victoria University of Wellington. 


\section{Table of Contents}

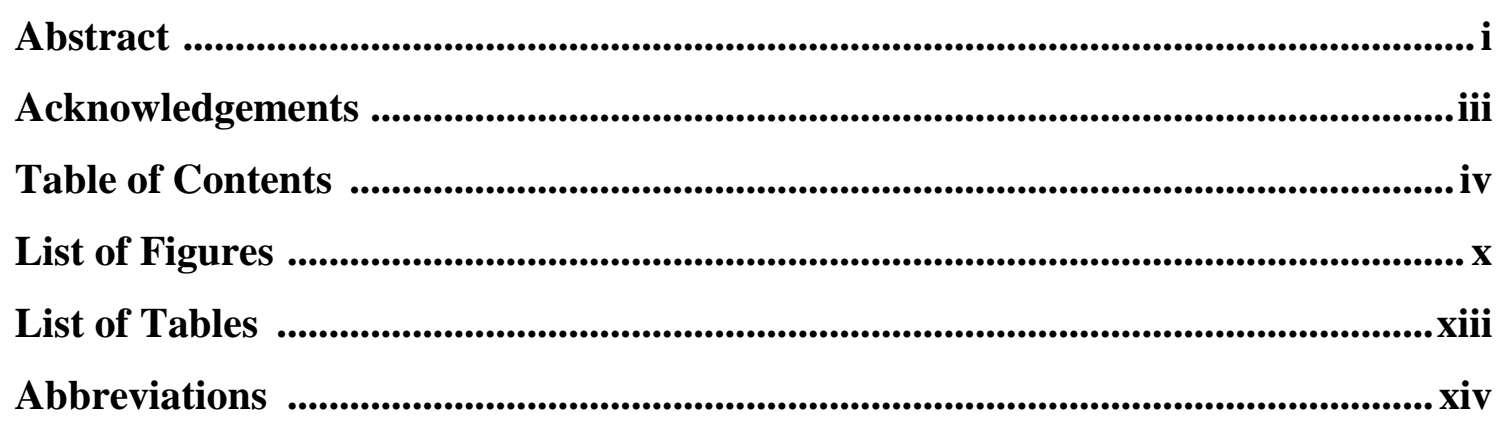

Chapter 1: Introduction.................................................................................................. 1

1.1 Drug addiction ...................................................................................................................... 1

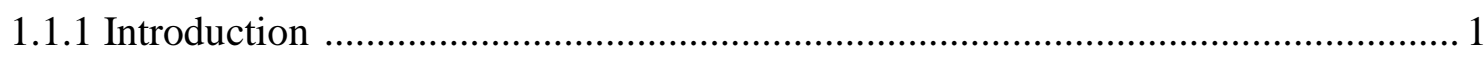

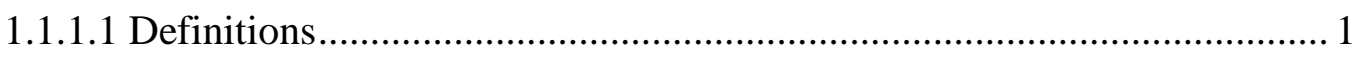

1.1.2 Drug addiction in New Zealand .................................................................. 2

1.1.3 The brain's natural reward pathway.............................................................. 3

1.1.4 Theories of drug addiction ............................................................................. 6

1.1.4.1 Classic opponent-process theory of addiction ………………………...... 6

1.1.4.2 Incentive-sensitisation theory of addiction ........................................... 7

1.1.5 Genetic susceptibilities to drug addiction ………….................................... 8

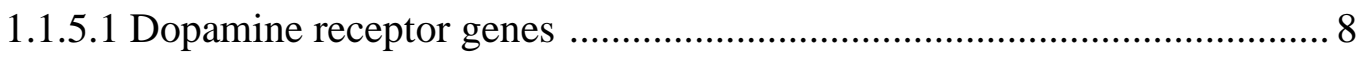

1.1.5.2 Dopamine transporter genes........................................................... 9

1.1.5.3 Serotonin transporter genes.............................................................. 9

1.1.5.4 Monoamine oxidase A genes .............................................................. 10

1.1.5.5 Catechol-O-methyl transferase genes ................................................... 10

1.1.6 Current therapeutics ............................................................................... 10

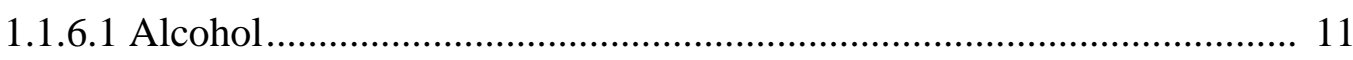

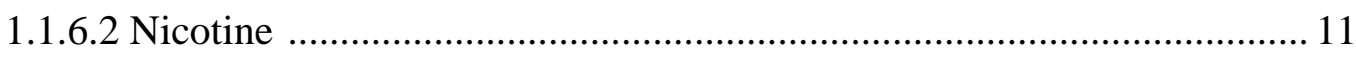

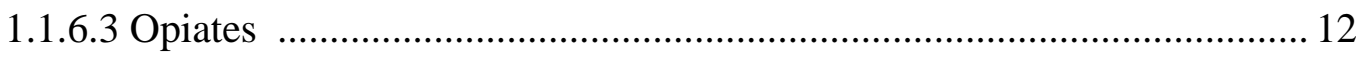

1.1.6.4 Amphetamines/Cocaine ................................................................... 13

1.2 Dopamine and the dopamine transporter................................................................... 14

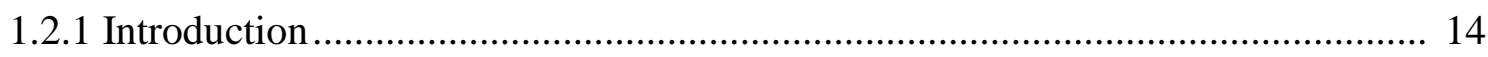

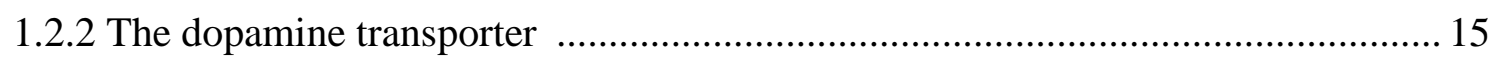

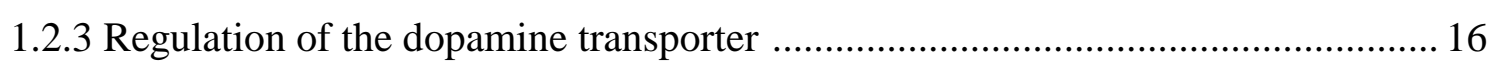

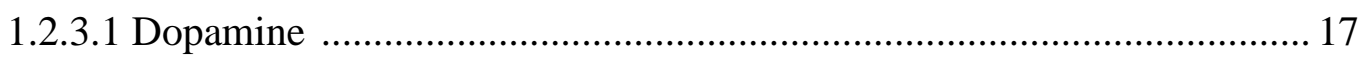




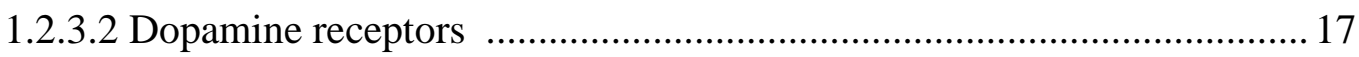

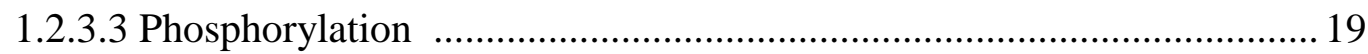

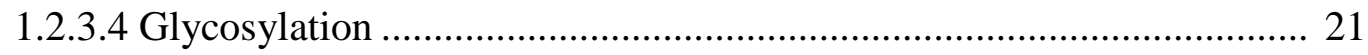

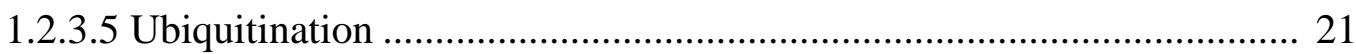

1.2.3.6 Drugs of abuse and dopamine signalling .......................................... 22

1.3 The Serotonin Transporter .................................................................................................. 30

1.4 The Kappa Opioid Receptor ...................................................................................... 31

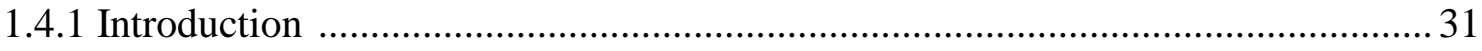

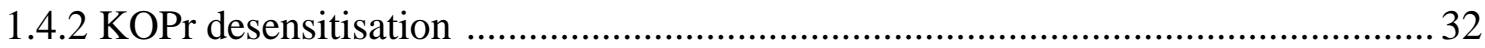

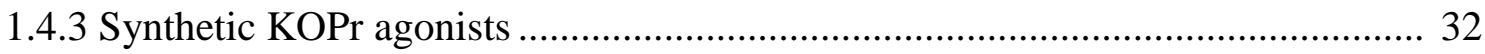

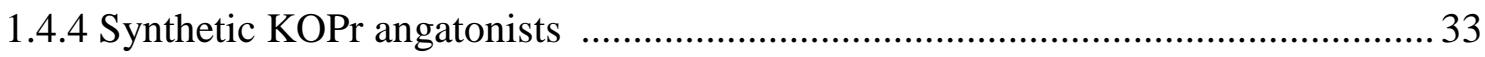

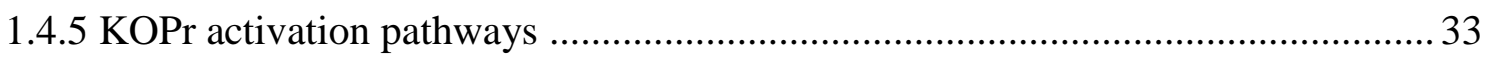

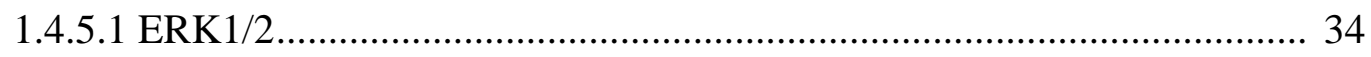

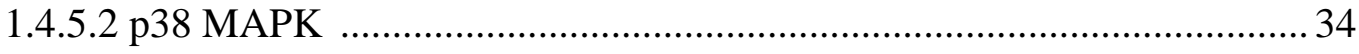

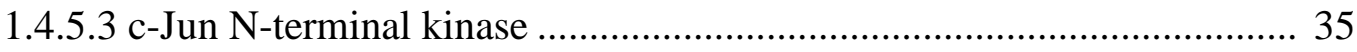

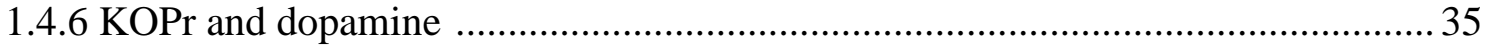

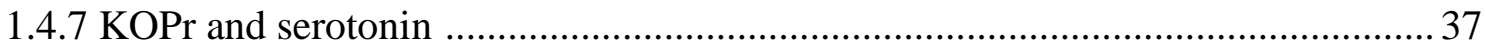

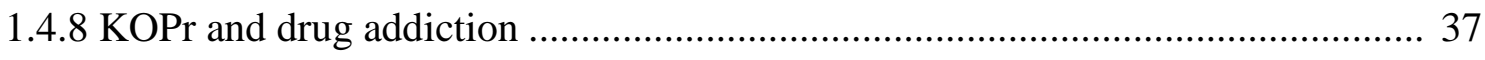

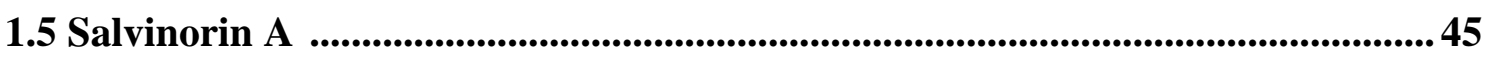

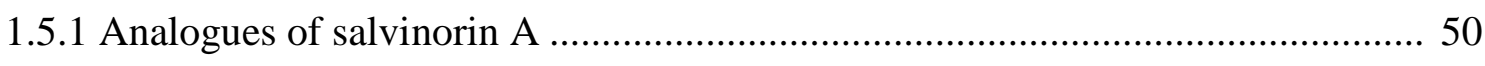

1.6 Aims and Objectives

Chapter 2: General methods ........................................................................... 53

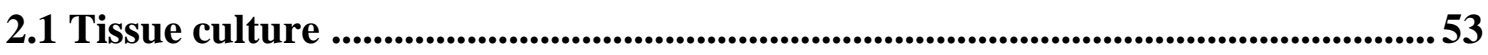

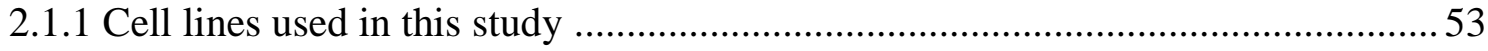

2.1.1.1 Human embryonic kidney cells (HEK-293) ......................................... 53

2.1.1.2 Green monkey kidney cells (COS7) ….............................................. 53

2.1.1.3 Mouse neuroblastoma cells (N2A) ................................................ 54

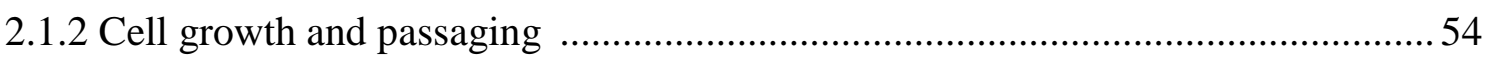

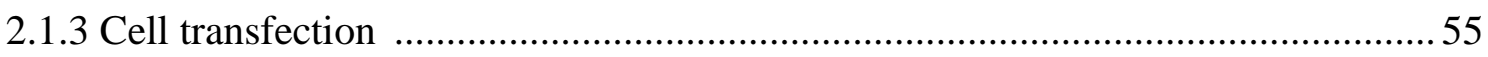

2.2 Plasmid Preparation ......................................................................................................... 55

2.2.1 Transformation of MAX Efficiency ${ }^{\circledR}$ DH5 $\alpha^{\mathrm{TM}}$ Competent Cells ........................ 55

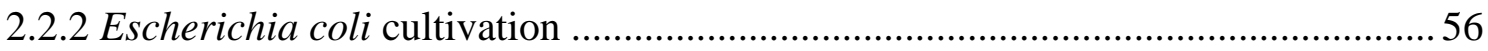

2.2.3 Plasmid purification using the Qiagen HiSpeed Maxi Kit ..................................56

2.3 Cell surface biotinylation ......................................................................5 58 
2.4 SDS polyacrlyamide gel electrophoresis and Western blotting ............................ 59

2.5 trans-4-[4-(dimethylamino)styryl]-1-methylpyridinium $\left(\mathrm{ASP}^{+}\right)$uptake ......... 62

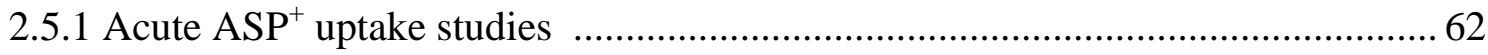

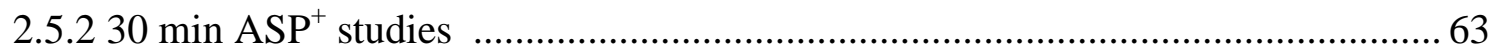

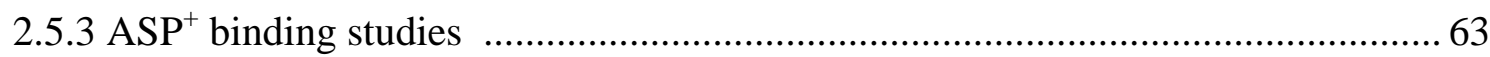

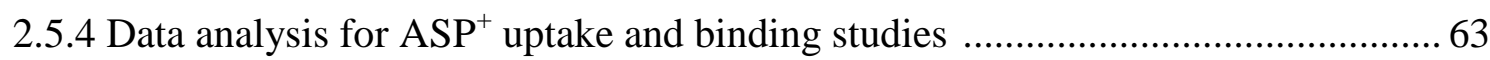

2.6 Total internal reflection fluorescence microscopy (TIRFM) ........................... 64

\section{Chapter 3: Effect of KOPr agonists on the function of DAT and} SERT in minced brain tissue from the rat .........................66

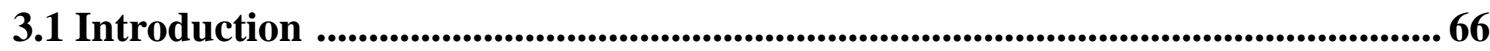

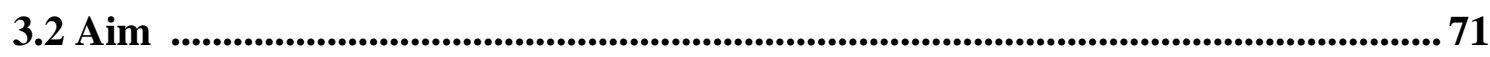

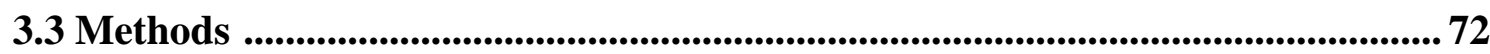

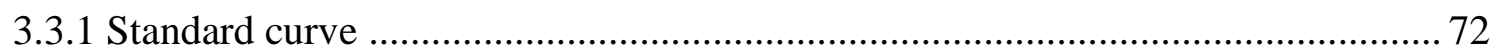

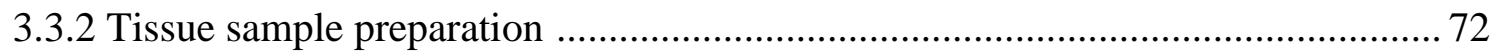

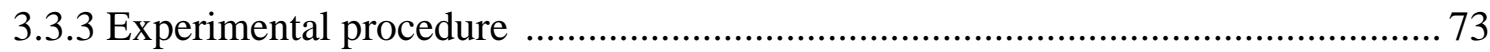

3.3.4 RDEV with GFP-hSERT transfected HEK-293 cells ......................................... 74

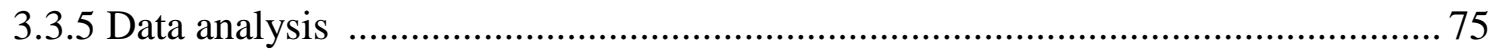

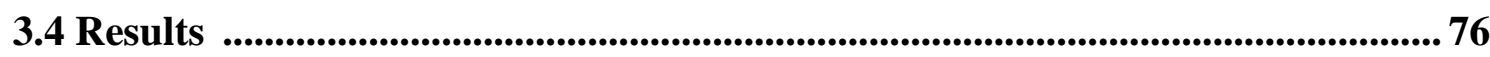

3.4.1 Effects of KOPr agonists on DAT function in brain tissue samples

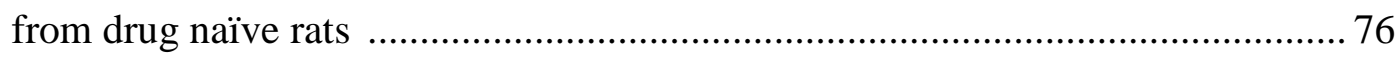

3.4.2 The effect of KOPr agonists on DAT function is reversed by the KOPr

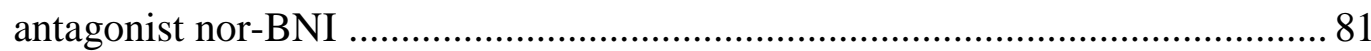

3.4.3 Establishment of RDEV for measurement of SERT in cells transfected with

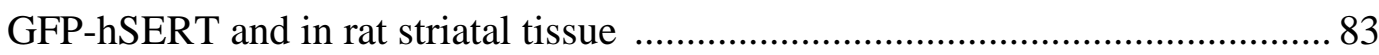

3.4.3.1 Transfected cell model .................................................................... 83

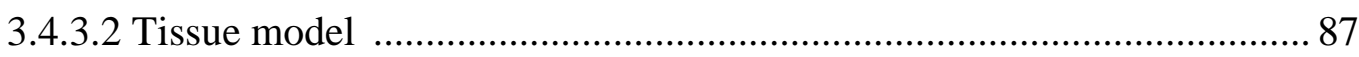

3.4.4 The effect of KOPr agonists on SERT function in the striatum .......................... 92

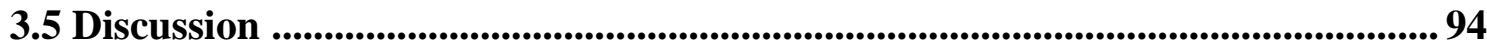

3.5.1 The effect of acute DA additions in the striatum, NAc and medial PFC ............. 95

3.5.2 The kinetics of DAT after addition of KOPr agonists in rat NAc tissue .............. 96

3.5.3 The effect of KOPr agonists on DAT function is nor-BNI reversible in

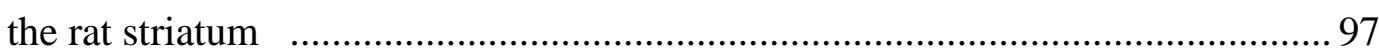

3.5.4 Establishing RDEV to measure SERT function in rat striatum and cells 


\section{Chapter 4: Effects of KOPr agonists on the function and} expression of DAT in a cell system

4.1 Introduction 105

4.1.1 4-(4-(dimethylamino)-styrl)- $N$-methylpyridinium $\left(\mathrm{ASP}^{+}\right)$uptake 105

4.1.2 Total internal reflection fluorescence microscopy (TIRFM) 107

4.1.3 Cell surface biotinylation 108

4.2 Aim 110

4.3 Methods 111

4.3.1 Confocal microscopy 111

4.3.2 Cell surface biotinylation 111

4.3.3 Total internal reflection fluorescence microscopy 111

4.3.4 Cell staining to visualise myc-rKOPr 111

4.4 Results

4.4.1 Initial considerations

4.4.1.1 Co-expression of YFP-hDAT and myc-rKOPr in HEK-293, N2A, and COS7 cells

4.4.1.2 $\mathrm{ASP}^{+}$uptake method validation 112

4.4.2 Acute changes in the function of DAT due to treatment with

KOPr agonists

4.3.2.1 The effect of KOPr agonists in different cell lines 120

4.4.3 The effect of a 30 min incubation with $\mathrm{KOPr}$ agonists on $\mathrm{ASP}^{+}$uptake 123

4.4.4 Acute cell surface expression studies

4.4.4.1 $\mathrm{ASP}^{+}$binding studies

4.4.4.2 Cell surface biotinylation studies

4.4.4.3 Total internal reflection fluorescence microscopy

4.4.5 Chronic effects of KOPr agonists on cell surface expression of YFP-hDAT

4.5 Discussion

4.5.1 Preliminary experiments 136

4.5.2 Functional changes 


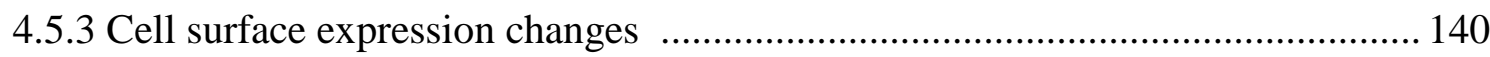

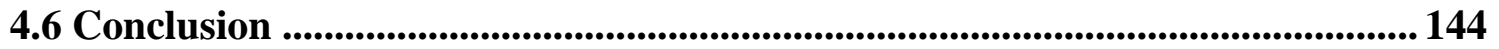

Chapter 5: Involvement of the ERK1/2 signalling pathway and lipid rafts on the modulation of DAT by KOPr ................ 145

5.1 Introduction .................................................................................................................. 145

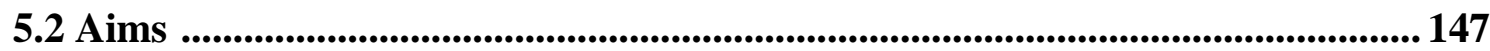

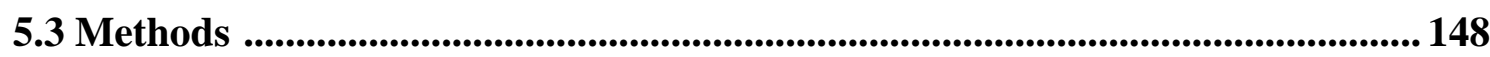

5.3.1 Western blotting for total protein ................................................................... 148

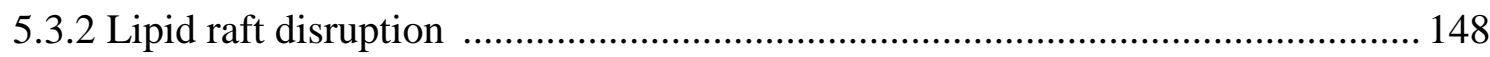

5.3.3 Fluorescence recovery after photo-bleaching (FRAP) ..................................... 148

5.3.3.1 Experimental protocol .................................................................... 148

5.3.3.2 Data analysis ................................................................................. 148

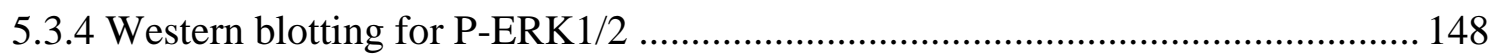

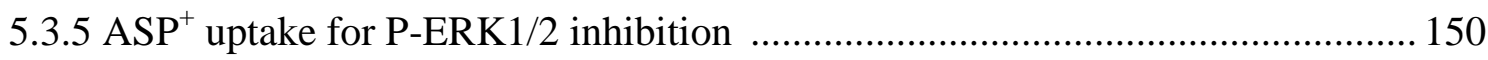

5.3.6 Biotinylation and TIRFM for P-ERK1/2 inhibition .......................................... 150

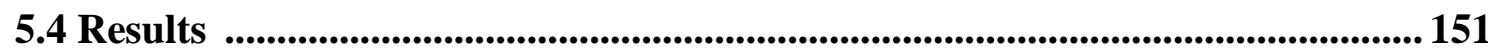

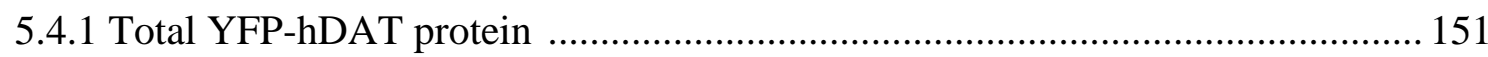

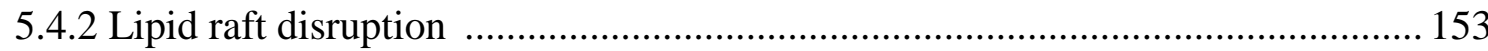

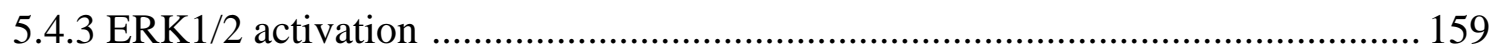

5.4.4 ERK1/2 activation and cell surface expression of YFP-hDAT ……………....... 170

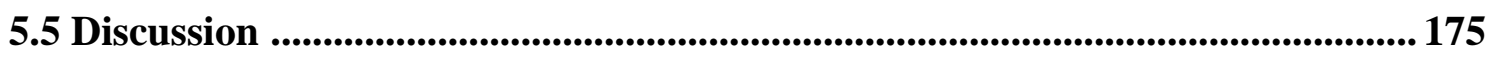

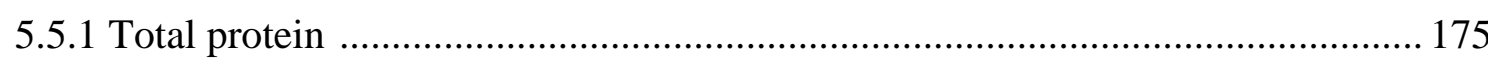

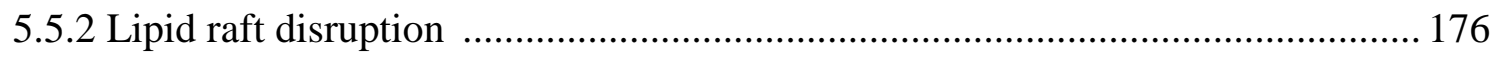

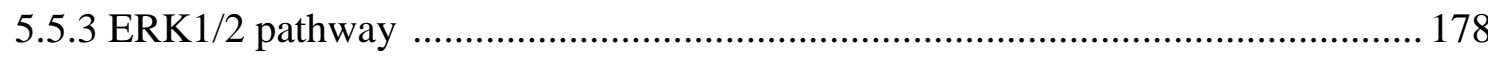

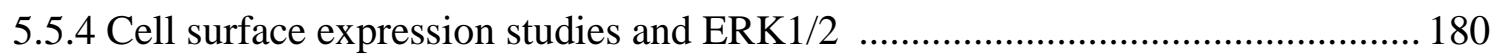

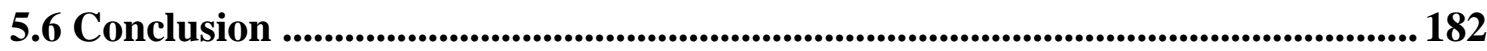

Chapter 6: Final Discussion ..............................................................183

6.1 Significance and key findings ....................................................................................... 183

6.1.1 Significance ………………………………………………………………... 183

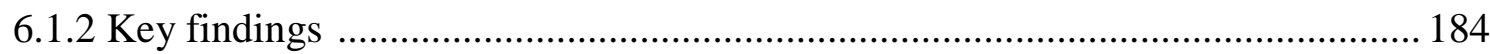

6.1.2.1 KOPr modulates DAT and SERT in isolated rat brain tissue ............. 184

6.1.2.2 KOPr modulates DAT in cells co-transfected with YFP-hDAT 
and myc-rKOPr

6.1.2.3 KOPr modulation of the cell surface expression of DAT 186

6.1.2.4 KOPr modulates DAT through ERK1/2, is dependent on lipid rafts and does not lead to increased total DAT protein

6.2 Future Directions 190

6.3 Overall significance 192

Appendix 1: Solutions 193

Appendix 2: Chapter 4 198

A2.1 $\mathrm{ASP}^{+}$uptake: Time of maximal effect 198

A2.2 Transfection optimisation of $\mathrm{N} 2 \mathrm{~A}$ and $\mathrm{COS7}$ cells 198

A2.3 Setting up TIRFM .201

Appendix 3: Chapter 5 203

A3.1 ERK1/2 optimisation .203

A.3.1 Serum starvation time 203

A3.2.2 Incubation time with P-ERK1/2 antibody 203

A3.2.3 Protein amount 205

References 206 


\section{List of Figures}

Figure 1.1: The brain's natural reward pathway ....................................................... 5

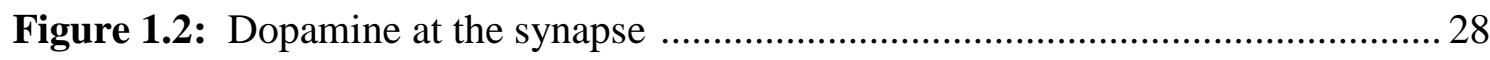

Figure 1.3: Important glycosylation, phosphorylation, ubiquitination and binding sites for DA and cocaine on the rat and human DAT .................................... 29

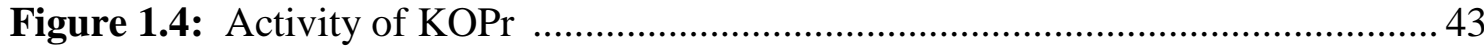

Figure 1.5: Structures of the classic KOPr agonists U50,488H (A) and U69593

(B) and antagonists nor-BNI (C), JDTic (D), and GNTI (E) 44

Figure 1.6: Structures of salvinorin A (A) and its analogues DS-1-240 (B) and DS-3-216 (C) 44

Figure 3.1: Effect of KOPr agonists on DA uptake in the striatum, nucleus accumbens, and medial prefrontal cortex .78

Figure 3.2: The effect of KOPr agonists on DAT kinetics in the nucleus accumbens

Figure 3.3: The effect of $100 \mathrm{nM}$ salvinorin $\mathrm{A}$ on uptake of $2 \mu \mathrm{M}$ DA

in the rat nucleus accumbens 80

Figure 3.4: Nor-BNI inhibition of the effects of KOPr agonists on DAT function in the rat striatum 82

Figure 3.5: Uptake of 5-HT in non-transfected and GFP-hSERT transfected HEK-293 cells 84

Figure 3.6: Ion and temperature dependence of 5-HT uptake in HEK-293 cells transfected with GFP-hSERT 85

Figure 3.7: The effect of anisomycin on SERT function in cells transiently transfected with GFP-hSERT …......................................................... 86

Figure 3.8: Inhibition of 5-HT uptake of DAT by GBR12909 .................................. 89

Figure 3.9: Ion and temperature dependence of 5-HT uptake in rat striatal tissue ...... 89

Figure 3.10: The effect of anisomycin on SERT function in rat striatal tissue. ........... 90

Figure 3.11: The effect of the SERT inhibitor fluoxetine on 5-HT uptake ................. 90

Figure 3.12: Uptake of 5-HT by SERT in the rat striatum and in frozen tissue

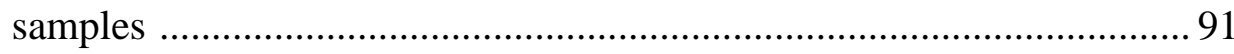

Figure 3.13: The effect of U50,488H on SERT function at 4, 10, and $15 \mathrm{~min}$........... 93

Figure 3.14: KOPr agonists decrease 5-HT uptake in the rat striatum ....................... 93

Figure 4.1: Binding and uptake of $\mathrm{ASP}^{+}$to YFP-hDAT HEK-293 cells .................... 106

Figure 4.2: Comparison of DIC (A), confocal (B), epi-fluorscence (C) and TIRF (D) microscopy 
Figure 4.3: Co-expression of YFP-hDAT and myc-rKOPr in cell lines used in this study

Figure 4.4: $\mathrm{ASP}^{+}$uptake and YFP intensity have a positive correlation in HEK-293 cells

Figure 4.5: Time course of $\mathrm{ASP}^{+}$uptake by HEK-293 cells transiently transfected with YFP-hDAT and myc-rKOPr

Figure 4.6: Changes in $\mathrm{ASP}^{+}$uptake after KOPr agonist addition is concentration- dependent and inhibited by nor-BNI and PTX 118

Figure 4.7: nor-BNI and PTX have no effect on uptake of $\mathrm{ASP}^{+}$on their own 119

Figure 4.8: Addition of $10 \mu \mathrm{M}$ of KOPr agonists to COS7 cells causes an increase in $\mathrm{ASP}^{+}$uptake that is reversible by nor-BNI and PTX

Figure 4.9: Addition of $10 \mu \mathrm{M}$ of KOPr agonists to N2A cells causes an increase in $\mathrm{ASP}^{+}$uptake that is reversible by nor-BNI and PTX

Figure 4.10: Long-term effects of KOPr agonists on $\mathrm{ASP}^{+}$uptake in HEK-293 cells

Figure 4.11: KOPr agonists cause no changes in binding of $\mathrm{ASP}^{+}$to YFP-hDAT

Figure 4.12: KOPr agonists do not alter cell surface expression of YFP-hDAT at short time points

Figure 4.13: KOPr agonists do not alter cell surface expression of YFP-hDAT at short time points as measured by TIRFM

Figure 4.14: A $30 \mathrm{~min}$ incubation with $\mathrm{U} 50,488 \mathrm{H}$ does not change cell surface expression of YFP-hDAT

Figure 4.15: A $30 \mathrm{~min}$ incubation with salvinorin A led to a significant increase in cell surface expression of YFP-hDAT

Figure 4.16: A 30 min incubation with U69,593 led to a significant increase in cell surface expression of YFP-hDAT

Figure 4.17: A 30 min incubation with DS-1-240 does not change cell surface expression of YFP-hDAT

Figure 4.18: A 30 min incubation with DS-3-216 led to a significant increase in cell surface expression of YFP-hDAT

Figure 5.1: Treatment for $30 \mathrm{~min}$ with KOPr agonists has no effect on total YFP-hDAT protein in HEK-293 cells

Figure 5.2: Effect of $\mathrm{M} \beta \mathrm{C}$ in non-transfected and transfected cells on $\mathrm{ASP}^{+}$ uptake 
Figure 5.3: FRAP in HEK-293 cells expressing YFP-hDAT and myc-rKOPr with and without $10 \mathrm{mM} \mathrm{M} \beta \mathrm{C}$

Figure 5.4: Lipid raft disruption attenuates the KOPr agonist-induced increase in DAT function

Figure 5.5: Effect of the MEK1/2 inhibitor U0126 on untreated HEK-293

cells 161

Figure 5.6: U50,488H modulates DAT function through an ERK1/2 dependent pathway 162,163

Figure 5.7: Salvinorin A modulates DAT function through an ERK1/2 dependent pathway 164,165

Figure 5.8: DS-1-240 modulates DAT function through an ERK1/2 dependent pathway 166,167

Figure 5.9: DS-3-216 modulates DAT function through an ERK1/2 dependent pathway 168,169

Figure 5.10: The effect of U0126 on the cell surface expression of YFP-hDAT in vehicle-treated HEK-293 cells 172

Figure 5.11: U0126 does not inhibit the increase in cell surface expression induced by $\mathrm{U} 69,593$ 173

Figure 5.12: U0126 inhibits the salvinorin A-induced increase in cell surface expression 174

Figure A2.1: Time of maximal effect of KOPr agonists on $\mathrm{ASP}^{+}$uptake 199

Figure A2.2: N2A and COS7 cells transfected with YFP-hDAT and myc-rKOPr with three different protocols 200

Figure A2.3: DA causes increased YFP-hDAT cell surface expression 202

Figure A3.1: Comparison between basal P-ERK1/2 levels after serum starvation for $30 \mathrm{~min}$ (left band) and $4 \mathrm{~h}$ (right band) 203

Figure A3.2: Comparison between a $1 \mathrm{~h}$ incubation with P-ERK1/2 antibody and a $20 \mathrm{~h}$ incubation 204

Figure A3.3: Comparison between $25 \mu \mathrm{g}$ of protein lysate and $50 \mu \mathrm{g}$ of protein lysate 205 


\section{List of Tables}

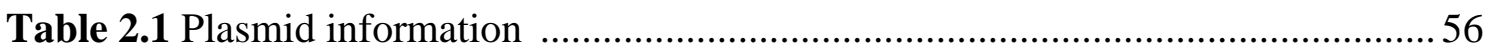

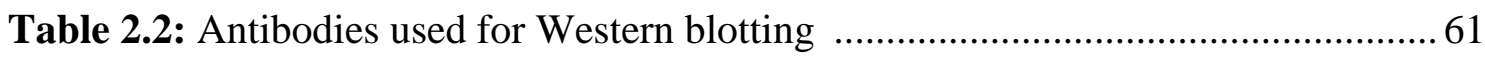

Table 3.1: Uptake kinetics of DAT in the nucleus accumbens …............................... 80

Table 3.2: Kinetic data from RDEV for control and anisomycin treated cells ............ 86

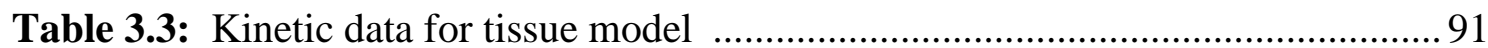

Table 4.1: Time taken for compounds to have their maximal effect on $\mathrm{ASP}^{+}$

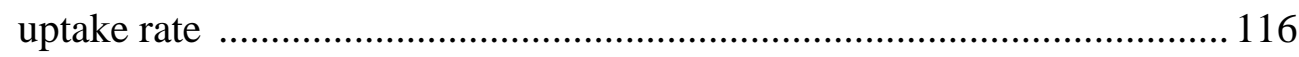

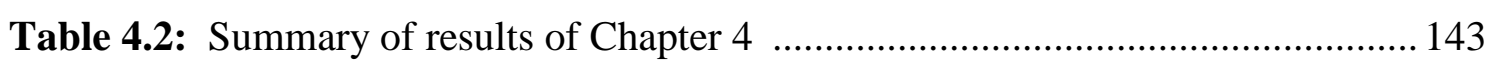

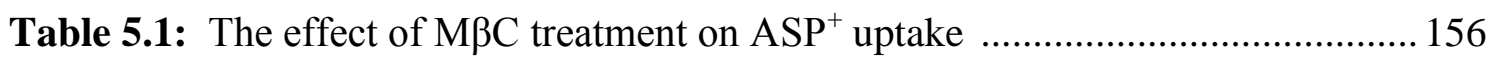

Table 6.1: Summary of key findings in this study .................................................. 189 


\section{Abbreviations}

5-HT

AFU

$\mathrm{ASP}^{+}$

CaMKII

cAMP

$\mathrm{CB}_{1}$

$\mathrm{CB}_{2}$

$\mathrm{CHO}$

COMT

COS7

CRF

DA

DAG

DAT

DMEM

DMSO

DOB

DOPr

DRD2

DRD2L

DRD2S

DRD3

DRD4

ERK1/2

FDA

FCS

FRAP

FSCV

GABA

GFP-hSERT

GNTI

GPCR
Serotonin (5-Hydroxytryptophan)

Arbitrary fluorescent unit

4-(4-(dimethylamino)-styrl)- $N$-methylpyridinium

$\mathrm{Ca}^{2+} /$ calmodulin-dependent kinase II

Cyclic adenosine monophosphate

Cannabinoid receptor 1

Cannabinoid receptor 2

Chinese hamster ovary cells

Catechol-O-methyl transferase

Green monkey kidney cells

Corticotrophin-releasing factor

Dopamine

Diacylglycerol

Dopamine transporter

Dulbecco's Modified Eagle's Medium

Dimethyl sulfoxide

4-Bromo-2,5-dimethoxy-phenylisopropylamine

Delta opioid receptor

Dopamine receptor D2

Dopamine receptor D2 long

Dopamine receptor D2 short

Dopamine receptor D3

Dopamine receptor D4

Extracellular-signal regulated kinase 1/2

Food and drug association

Fetal calf serum

Fluorescence recovery after photobleaching

Fast scan cyclic voltammetry

$\gamma$-Aminohydroxybutyric acid

Green fluorescent protein tagged-human serotonin transporter

5'-Guanidinonaltrindole

$\mathrm{G}$ protein coupled receptor 


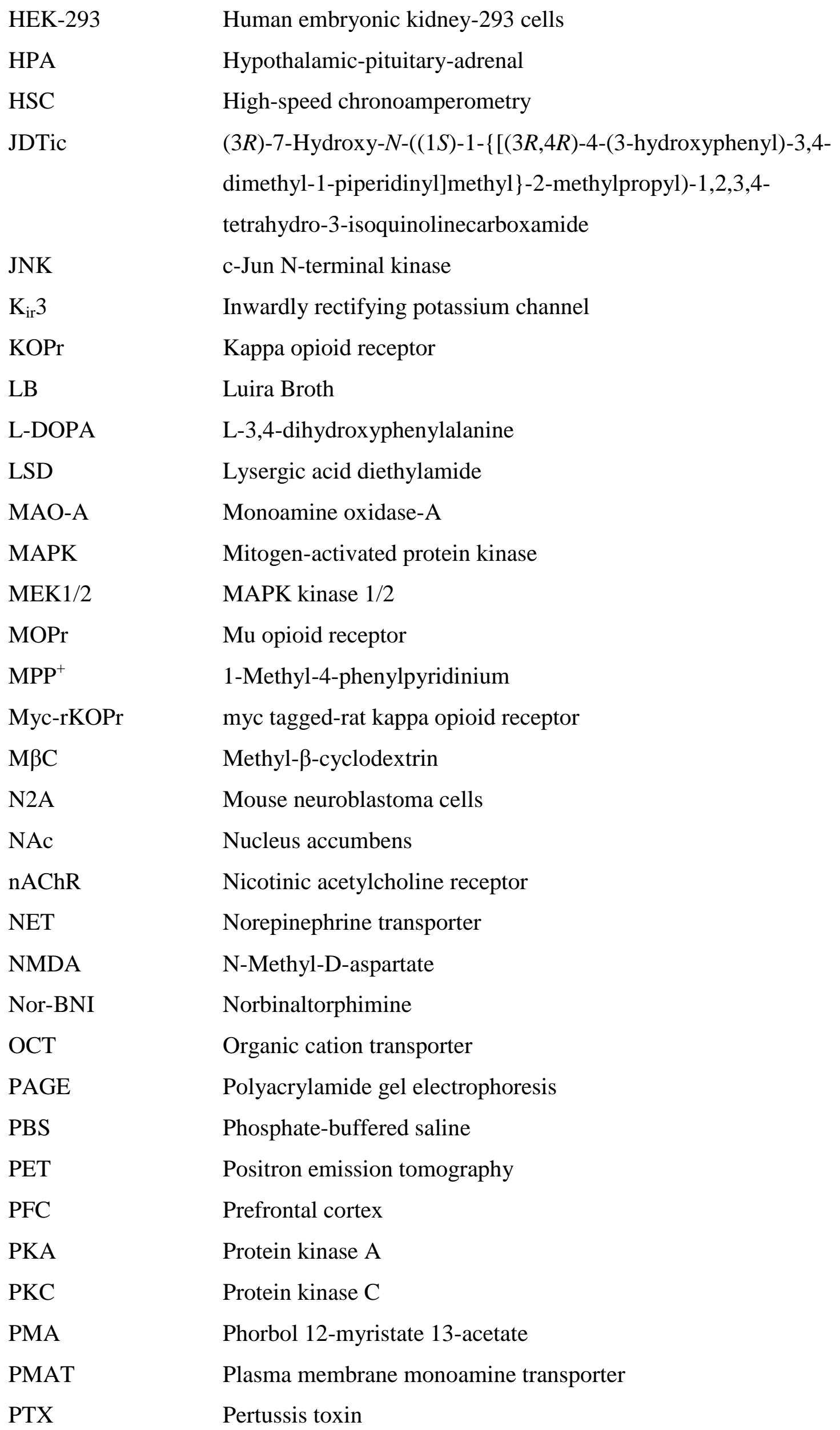


PVDF

RDEV

RFLP

ROI

RPMI

SDS

SERT

SN

SNP

SPECT

SSRI

TBS

TIRFM

U0126

$\mathrm{U} 50,488 \mathrm{H}$

$\mathrm{U} 69,593$

VMAT

VNTR

VTA

YFP-hDAT
Polyvinylidine difluoride

Rotating disk electrode voltammetry

Restriction fragment length polymorphism

Region of interest

Roswell Park Memorial Institute

Sodium dodecyl sulphate

Serotonin transporter

Substantia nigra

Single nucleotide polymorphism

Single-photon emission computed tomography

Selective serotonin reuptake inhibitors

Tris-buffered saline

Total internal reflection fluorescence microscopy

1,4-Diamino-2,3-dicyano-1,4-bis (o-

aminophenylmercapto)butadiene

(Trans-( \pm )-3,4-dichloro-N-methyl-N-(2-[1-pyrrolidinyl]-

cyclohexyl) benzeneacetamide methanesulfonate

(5 $\alpha, 7 \alpha, 8 \beta)-(+)-N-m e t h y l-N-(7-(1-p y r r o l i d i n l y)-1-$

oxaspiro(4,5)dec-8-yl)benzeneacetamide

Vesicular monoamine transporters

Variable number tandem repeat

Ventral tegmental area

Yellow fluorescent protein tagged-human dopamine transporter 


\section{Chapter 1: Introduction}

Drug abuse and addiction are major social and economic burdens world-wide. Currently there are limited therapeutics available to combat drug addiction, with none available to treat psychostimulant abuse. The dopamine transporter (DAT) is involved in the process of addiction and the kappa opioid receptor (KOPr) has been shown to modulate DAT function. Therefore the design of novel KOPr agonists may lead to potential anti-addiction therapies through modulation of DAT. This study investigates the unique KOPr agonist salvinorin A and novel analogues based on salvinorin A's structure.

\subsection{Drug addiction}

\subsubsection{Introduction}

Drug addiction, also called substance dependence, is a chronic relapsing brain disorder defined as the compulsion to seek and take drugs even when the adverse consequences are known to the individual (Koob and Kreek 2007). In New Zealand, it is estimated that $30 \%$ of the population abuse drugs such as nicotine, alcohol, psychostimulants and opioids (M.O.H. 2007) which in turn leads to large economic and social costs. Most drugs of abuse currently have no successful long-term therapies, with studies showing that $80-90 \%$ of human addicts relapse back to drug taking behaviour, even after long periods of abstinence and with the aid of currently available pharmacotherapies (Reviewed in Shippenberg et al. 2007). Available therapeutics for drugs of abuse such as nicotine have very low success rates, and others such as methadone, used for opiate addiction, are highly addictive themselves. Currently there are no pharmacotherapies available for psychostimulants such as cocaine, amphetamine, and methamphetamines. There is therefore a need for development of better pharmacotherapies that have fewer side effects and greater long term success rates.

\subsubsection{Definitions}

Drug abuse and drug addiction are defined as two separate disorders, with drug abuse the less debilitating of the two. For a patient to be diagnosed with drug abuse syndrome they need to display repeated and clinically significant adverse consequences related to frequent substance use. These consequences include: failing to fulfil major roles or obligations due to drug use; use of drugs in physically hazardous situations; legal 
problems due to drug use; and continued use of a drug despite social or interpersonal problems (A.P.A. 2000). Drug abuse is often seen as a stepping stone to drug addiction.

For a patient to be diagnosed with drug addiction, they need to exhibit at least three of the following symptoms: symptoms of tolerance; symptoms of withdrawal; the use of the substance for longer time periods or in larger amounts then intended; persistent desire, or inability to decrease or control use; the spending of larger periods of time in efforts to obtain the substance; a reduction in social, occupational, or recreational activities because of drug use; and continued use of the substance with the knowledge of health, social or economic problems (A.P.A. 2000).

Sensitisation to a drug of abuse often occurs during early intermittent drug taking and is the process in which there is an increase in the expected effect of a drug after repeated use, or hypersensitivity to the effect of the drug. This is often seen as locomotor sensitisation with psychostimulants such as cocaine in laboratory rats (Shippenberg and Rea 1997). Sensitisation is one of the factors involved in drug cravings and relapse. Tolerance occurs after long term drug use, and is characterised by a blunted effect of the drug that leads to increased doses used.

Cravings and withdrawal are interlinked. Craving is a psychological dependence, i.e. an intense desire to re-experience the effects of a drug. Withdrawal is a group of physiological and psychological factors that occur immediately after attenuation of drug taking. Craving and withdrawal are also cue-induced and play large roles in relapse.

\subsubsection{Drug addiction in New Zealand}

The drugs used and abused in New Zealand can be quite unique from those abused in other parts of the world. This is thought to be because of the isolation of New Zealand and the country's strict border control, which makes it harder to get drugs such as cocaine and opiates into the country (Braye et al. 2007). There have been a number of surveys carried out over the past 10 years highlighting the fact that New Zealand has a high prevalence of alcohol, tobacco, cannabis and amphetamine use (M.O.H. 2007). A mental health survey carried out in 2003 included a section on substance use disorders, in which the authors looked at both abuse and dependence of substances as defined by the American Psychiatric Association. Of the 55,000 people surveyed, 12.7\% had experienced a substance use disorder in their lifetime, with young male Maori the most 
common demographic. These statistics did not include tobacco use (20\% of people surveyed smoked) or alcohol abuse (2.6\% abuse and 1.3\% dependence). The survey also highlighted the high co-morbidity of substance use disorders with alcohol abuse and dependence, and mental health issues (Wells et al. 2007).

In 2003 a New Zealand Ministry of Health survey found the top five most commonly used drugs in New Zealand were alcohol, tobacco, cannabis, amphetamines, and ecstasy (MDMA) (M.O.H. 2007). An earlier survey carried out to investigate the use of amphetamine-type stimulants in New Zealand found that 1 in 10 people surveyed over the age of 18 had tried amphetamine-type stimulants, with one third of these being frequent users (Wilkins et al. 2004). Methamphetamine, also known as 'P', is a more recent amphetamine-type stimulant abused in New Zealand and appears to be growing in popularity. This drug has greater appeal to young professionals, with users predominantly being young male Europeans (Wilkins et al. 2006). There is also a population of intravenous opiate users in New Zealand, with estimates of about 10,000 in 2008. Of these, there were 4,608 who were on a methadone program (Deering et al. 2008). Another study published in 2006 found that $76.6 \%$ of 27 year olds had used cannabis in their lifetime, with $12.5 \%$ meeting the criteria for cannabis dependence. A further 3.6\% were also dependent on other illegal substances (Boden et al. 2006).

Drug use often leads to criminal behaviour, and a study carried out by the New Zealand police in 2005, in which they tested arrestees for illegal drugs in their system, found that of the arrestees that provided usable urine samples, $71 \%$ were positive for illegal drugs. Of those, $48 \%$ reported using drugs at the time of their arrest, $36 \%$ reported use of alcohol, $18 \%$ reported cannabis use, and $6 \%$ reported methamphetamine use (Hales and Manser 2007).

These surveys highlight that drug abuse and dependence are major issues in New Zealand, with yearly costs estimated to be $\$ 16.5$ billion (Slack et al. 2009). Therefore, there is a need for further research into drug addiction and potential therapeutics to decrease drug use.

\subsubsection{The brain's natural reward pathway}

The brain's natural reward pathway is activated during rewarding events such as eating, drinking, and sex (Adinoff 2004). Drugs of abuse act on this pathway, overwhelming 
the system and leading to dysregulation of natural reward systems. This pathway is known as the mesocorticolimbic pathway. Dopaminergic neurons make up the core of this pathway, with inhibitory and excitatory systems, and other monoamine systems feeding into it. Dopaminergic neurons have cell bodies that are found in the ventral tegmental area (VTA) that project forward to the nucleus accumbens (NAc) and prefrontal cortex (PFC) via the amygdala (Figure 1.1). The NAc is the brain's reward centre, and the PFC is involved in 'executive functions' such as distinguishing between good and bad, the consequences of actions, setting goals, and predicting outcomes (Robinson and Berridge 2000). The amygdala is involved in assigning reward values to stimuli and in memory consolidation for emotionally arousing events (Robinson and Berridge 2000). Dopaminergic neurons with cell bodies present in the substantia nigra project forward to the striatum, a brain region involved in motor control and habit learning (Robinson and Berridge 2000). This pathway is known as the nigrostriatal dopamine pathway. Drugs of abuse hijack these pathways in the brain, leading to increased reinforcement compared to natural reinforcers. A microdialysis study carried out by Hernandex and Hoebel (1988) demonstrates this increase in reinforcement. Microdialysis was used to measure extracellular dopamine (DA) levels in freely moving rats that were able to push a lever for food rewards, to receive electrical self-stimulation or to receive amphetamine or cocaine infusions. Food reward increased extracellular DA concentrations by 37\%, which was found to be the same for self-stimulation with or without a food reward. Amphetamine and cocaine both caused large-scale increases in extracellular DA in the NAc, 5-fold higher than natural levels, clearly demonstrating the increased reinforcing nature of drugs of abuse compared to normal rewards.

Dopamine is also involved in the tuberoinfundibular pathway that consists of dopaminergic neurons that project from the hypothalamus to the pituitary gland. This pathway regulates secretion of prolactin from the anterior pituitary gland and is involved in hormonal regulation and sensory processes (Steyn et al. 2007; Lyons et al. 2010). 


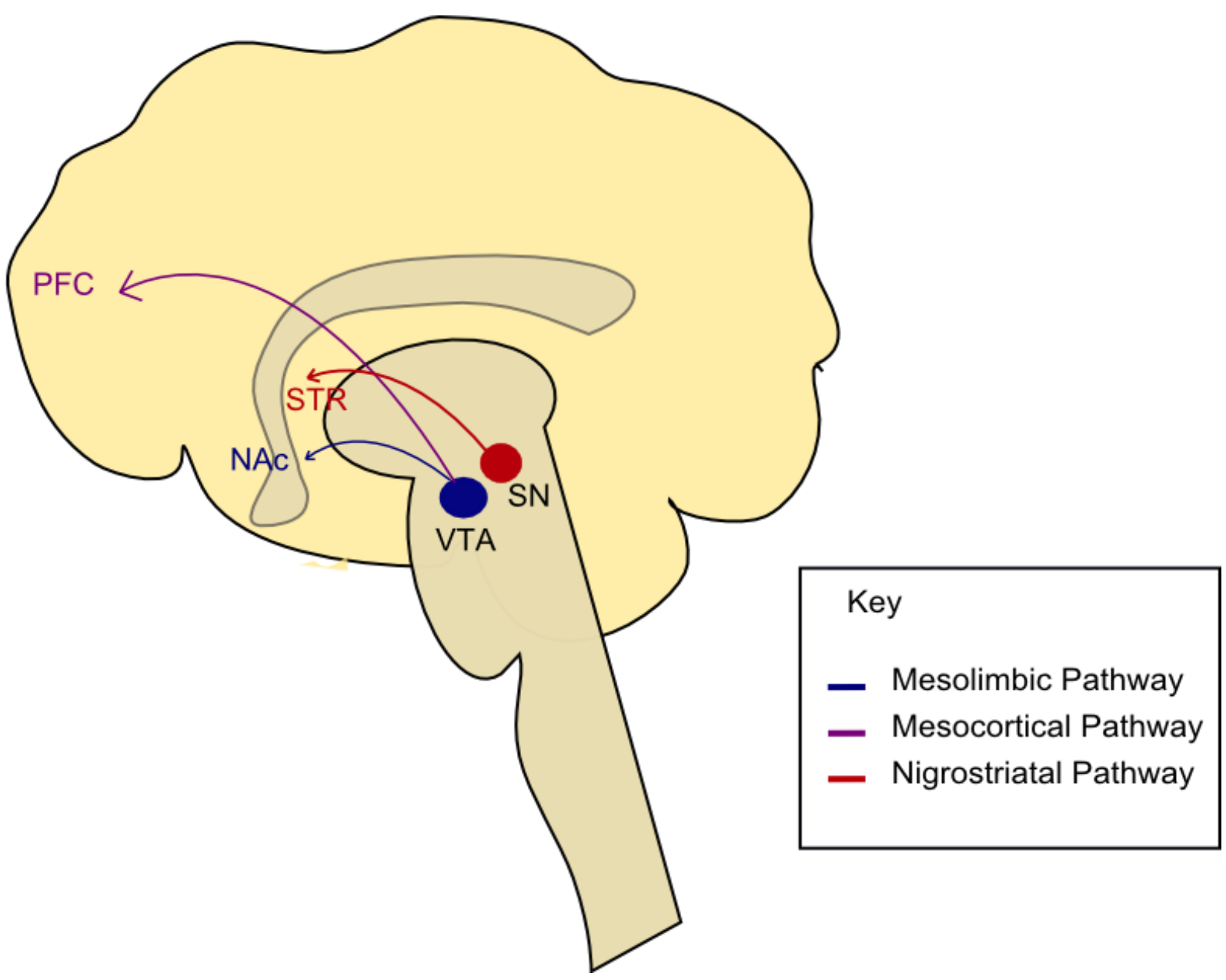

Figure 1.1: The brain's natural reward pathway. Dopaminergic neurons projecting from the ventral tegmental area (VTA) innervate the nucleus accumbens (NAc) and make up the mesolimbic pathway. Dopaminergic neurons projecting from the VTA also innervate the prefrontal cortex (PFC), making up the mesocortical pathway. Dopaminergic neurons with cell bodies in the substantia nigra (SN) project to the striatum (STR) and make up the nigrostriatal pathway. These dopaminergic pathways are all involved in natural reward and addiction. 


\subsubsection{Theories of drug addiction}

There are many theories of drug addiction, with no model on its own completely able to explain the complexities of the disorder. Outlined below are two of the more widely accepted models of addiction: the opponent-process theory and the incentivesensitisation theory. These theories of addiction are continually being remodelled as more information about drug addiction becomes available.

\subsubsection{Classic opponent-process theory of addiction}

This theory was first proposed by Solomon and Corbit in 1974 (Solomon and Corbit 1974). They postulated that there is a slow opposing process that counteracts the initial hedonic effects of a drug or its stimulus. This opposing effect increases over time, masking the hedonic effects of the drug or its stimulus. Initial drug taking causes a large hedonic effect with only a small opposing negative effect. As drug use increases, tolerance builds up, causing a decreased hedonic effect, and the negative effect increases as withdrawal and craving develop, changing the motivation of drug use from pleasure to prevention of dysphoric effects (Solomon and Corbit 1974).

The model has been extended over time as understanding has increased about the processes involved in addiction. The most recent version is Koob's theory of addiction, that includes homeostatic dysregulation (Koob and Le Moal 1997). The hypothesis in this model is that drug addiction involves both the neural reinforcement of the drug and the neuroadaptation of the reward and stress systems (Koob 2008). Together this leads to changes in motivational systems that cause a shift in the brain from homeostasis to allostasis, leading to addiction (Koob and Le Moal 2001). Allostasis in this case is defined as the process of maintaining stable reward function by changing the brain's reward mechanisms, effectively resetting the system parameters to a new set-point to match the demand on the system (Koob and Le Moal 2001).

Koob's model suggests there are three stages from initial drug use to drug addiction. These stages form a "spiralling distress" cycle in which factors such as the DA system and the stress system play a role (Koob and Le Moal 1997). The first stage of this model is preoccupation/anticipation, in which there is a preoccupation with obtaining the drug and a persistent desire to use it. This involves conditioning of the positive and negative reinforcement of the drug that plays a role of sensitisation. The second component is the binge/intoxication stage, in which drug use is increased to a point 
whereby the drug is taken more often and in greater amounts than intended. This leads to the drug becoming a positive reinforcer, and it continues to be sought out because of the rewarding effects. After this stage it is hypothesized that there is a down-regulation of the reward pathway and an up-regulation of stress pathways within the brain. The final component is the withdrawal/negative effect and the formation of tolerance. Withdrawal and drug use begins to affect social and occupational activities, and the drug becomes a negative reinforcer and is taken to alleviate these negative effects. The hypothalamic-pituitary-adrenal (HPA) brain stress system, and the extra hypothalamic corticotrophin-releasing factor (CRF) brain stress system are involved in this step, leading to continued drug seeking, and often leading to relapse after long periods of abstinence (Koob and Kreek 2007). This model explains the process of relapse by postulating that after long periods of abstinence, the brain returns to a somewhat 'normal' homeostatic state, but that there is residual activation of the stress systems and underactivation of the reward system. This leads to vulnerability to stress and drug related cues that can lead to relapse (Koob and Le Moal 2001)

\subsubsection{Incentive-sensitisation theory of addiction}

The incentive sensitisation model of addiction is seen as the more contemporary view of addiction. This model separates 'wanting' and 'liking' and states that drug addiction occurs due to drug 'wanting', which can often be subconscious, rather than drug 'liking' or hedonic effects (Robinson and Berridge 1993). This model is based around four main points. The first is that potentially addictive drugs all share the ability to cause neuroadaptations in the brain in the form of persistent hypersensitivity (sensitisation) (Robinson and Berridge 2001). This occurs during intermittent drug use but does not occur in every individual, only in those who are more susceptible to drug addiction (Berridge and Robinson 1995). The second point is that the brain regions affected by sensitisation are those involved in reward and mediate drug 'wanting' or incentivesensitisation. Drug wanting is unconscious and is often paired with a drug related cue (Robinson and Berridge 2001). The third point is that repeated drug use sensitises the neural substrates involved in drug 'wanting' but not 'liking', and there is an increasing dissociation between 'wanting' and 'liking' (Berridge and Robinson 1995). Finally, this effect is not conscious, and often the individual is unaware of cravings for the drug (Berridge and Robinson 1995). This theory suggests that 'liking' or hedonic effects of drugs are not sensitised in addiction and are not mediated by the dopaminergic system, but that 'wanting' or incentive is sensitised by drug use and is mediated by the 
dopaminergic system (Robinson and Berridge 2001). This prolonged neural sensitisation leaves former addicts at risk of relapse if they are in situations where drug cues are present or if they do use the drug again, and in relapse their responses will be even greater than before (Robinson and Berridge 2000).

As DA and the brain's natural reward pathway play a central role in drug addiction, it is important to further understand the mechanisms of how drugs of abuse cause their effects and how they regulate DA levels in the synapse. While none of the theories of drug addiction can fully describe how all drugs of abuse function in the brain, it is a good starting point for understanding the different ways in which drugs can modulate brain pathways. It is also important to understand how pharmacotherapies for drug abuse work through these pathways so that future pharmacotherapies can be developed that have higher success rates and can be used for drugs which currently have no therapeutics available.

\subsubsection{Genetic susceptibilities to drug addiction}

As mentioned above, some individuals are more susceptible to drug addiction than others. Genetic variabilities are thought to be involved in increased vulnerability to drug addiction. Outlined below are some genetic variations that have shown links with increased susceptibility to general addictive behaviour. For a detailed review see Khokhar et al. (Khokhar et al. 2010).

\subsubsection{Dopamine receptor genes}

An important neurotransmitter in the brain that plays a major role in drug addiction is DA. Links between DA receptor genes and susceptibility to drug taking have been found. The gene for the D2 dopamine receptor (DRD2) has been linked to addiction to many different drugs. The DRD2 restriction fragment length polymorphism (RFLP) Taq1A has been shown to cause a decrease in DRD2 density in the brain of healthy volunteers (Pohjalainen et al. 1998; Jonsson et al. 1999), although this response has also been disputed (Laruelle et al. 1998). This polymorphism is a C>T substitution $10 \mathrm{~kb}$ from the 3' end of the DRD2 gene that has been found in the coding region of the novel serine/threonine kinase ankyrin repeat domain protein 1 (Neville et al. 2004). The polymorphism causes an amino acid substitution in the $11^{\text {th }}$ ankyrin repeat, which is thought to alter the function of the protein (Neville et al. 2004). This polymorphism has been associated with susceptibility to abuse of a wide range of different substances, 
although again there are conflicting reports. The Taq1A polymorphism has been associated with alcoholism (Noble et al. 2000), tobacco use (Huang et al. 2009a), psychostimulant abuse and polydrug use (Persico et al. 1996), and heroin use (Lawford et al. 2000). In heroin use, the polymorphism was also linked with decreased effectiveness of methadone treatment (Lawford et al. 2000).

Another DRD2 polymorphism, Taq1B, is a single nucleotide polymorphism (SNP) found $913 \mathrm{bp}$ upstream of exon 2 (Blum et al. 1993). The B1 allele has been associated with tobacco use (Spitz et al. 1998), psychostimulant polysubstance abuse (Persico et al. 1996), alcohol abuse (Blum et al. 1993), and cocaine dependency (Noble et al. 1993).

The dopamine receptor 3 (DRD3) functional polymorphism rs6280 substitutes serine residue 9 to a glycine and has been linked to increased nicotine dependence in European Americans (Huang et al. 2008). The dopamine receptor 4 (DRD4) L allele of the $48 \mathrm{bp}$ variable number tandem repeat (VNTR) polymorphism is linked to smoking in AfricanAmericans and has been associated with decreased cessation therapy success (Shields et al. 1998).

\subsubsection{Dopamine transporter genes}

The dopamine transporter (DAT) plays an important role in modulating DA levels within the reward pathway. The gene for DAT, SLC6A3, is found on chromosome 5 p15 and is about $64 \mathrm{kbp}$ long. Many genetic studies have been carried out to investigate if there are associations between polymorphisms of this gene and susceptibility to addiction. The 3 allele of a $30 \mathrm{bp} \mathrm{VNTR} \mathrm{in} \mathrm{intron} 8$ of SLC6A3 has been associated with cocaine abuse in a Brazilian population (Guindalini et al. 2006). Another polymorphism, a G to A substitution in the 3' untranslated region of SLC6A3 has been associated with a susceptibility to an early onset of smoking (Ling et al. 2004) and a 9 allele of the 3' VNTR of 40 bp polymorphism, which leads to decreased DAT expression, is linked with protection from smoking dependence (Lerman et al. 1999).

\subsubsection{Serotonin transporter genes}

The serotonin transporter (SERT) gene SLC64A is found on chromosome 17q11, and due to the SERT proteins involvement in mood disorders, the gene has been investigated for links to drug addiction susceptibility (Ramamoorthy et al. 1993). The short allele of SLC64A linked promoter region leads to decreased expression of the 
gene and decreased serotonin (5-HT) uptake and has been associated with alcohol dependence (Feinn et al. 2005) and heroin dependence (Gerra et al. 2004).

\subsubsection{Monoamine oxidase A genes}

Monoamine oxidase A (MAO-A) is an enzyme that functions to degrade monoamine neurotransmitters, specifically norepinephrine, epinephrine, 5-HT, and DA (Bach et al. 1988). The MAO-A gene is a sex-linked gene with a $30 \mathrm{bp}$ repeat sequence that is repeated 3, 3.5, 4, or 5 times $1.2 \mathrm{~kb}$ upstream of the coding sequence (Sabol et al. 1998). This polymorphism has been shown to be associated with smoking in male Chinese, with the 3 repeat being more common with smoking individuals then non-smoking (Jin et al. 2006). Other polymorphisms have also been associated with alcohol abuse in Chinese (Hsu et al. 1996) and Europeans (Vanyukov et al. 1995).

\subsubsection{Catechol-O-methyl transferase genes}

Catechol-O-methyl transferase (COMT) is an enzyme involved in degradation of catecholamines such as DA, norepinephrine, and 5-HT. A polymorphism in this gene at codon 158 codes for either a valine, which produces a high activity COMT enzyme, or a methionine, which produces a lower activity COMT (Vandenbergh et al. 1997). The high activity COMT is associated with susceptibility to polysubstance abuse (Vandenbergh et al. 1997), heroin abuse (Horowitz et al. 2000), and nicotine dependence (Beuten et al. 2006). Interestingly the lower activity COMT is associated with alcohol abuse (Tiihonen et al. 1999).

\subsubsection{Current therapeutics}

There are a number of different pharmacotherapies available for different drug addictions, particularly alcohol, nicotine, and opiates. These therapies in general have a relatively low long-term success rate. Currently there are no food and drug administration (FDA) approved pharmacotherapies to treat psychostimulant abuse, highlighting the need for an understanding of how these drugs function so that new treatments can be developed.

There are two different types of pharmacotherapies that can be developed to prevent drug use: therapies to attenuate drug taking and therapies to prevent relapse. Pharmacotherapies to attenuate drug seeking usually either block or imitate the effects of the drug of abuse, leading to a decrease in drug taking. Currently most available 
pharmacotherapies for drug addiction work in this manner. Two of the major factors that lead to drug relapse are stress and depression. Therefore, development of pharmacotherapies that can be taken long-term after initial drug taking has ceased may help to decrease the high rates of relapse to drug taking after long periods of abstinence. While there are currently no FDA approved anti-relapse drugs available, there have been promising clinical trials carried out in animal models (Reviewed in YahyaviFirouz-Abadi and See 2009)

\subsubsection{Alcohol}

There are several pharmacotherapies available for alcohol addiction. Nalmefene and naltrexone are opioid antagonists that block the 'high' gained from alcohol use. A meta-analysis study carried out investigating the success of naltrexone concluded that naltrexone is a successful short-term therapy for alcohol abuse, but not for long-term treatment (Srisurapanont and Jarusuraisin 2005). Acamprosate calcium (Campral) is a pharmacotherapy that is used to reduce the incidence of relapse on already abstinent alcoholics. Its mode of action is not known, but it appears to interact with N-methyl-Daspartate (NMDA) glutamate receptors in the brain and has been shown to have protective effects against glutamate toxicity in alcohol-exposed neural cultures (al Qatari et al. 2001). Campral does not affect the pharmacokinetics of alcohol in any way and therefore is not a cessation therapy as such, but is more of an 'anti-craving' therapy (Brasser et al. 2004). Disulfiram (Antabuse) is a drug that makes the patient sick if they drink alcohol. It also causes headaches and facial flushing and is used as a deterrent to alcohol drinking. As it is often reliant on the patient to administer it themselves it is therefore not always very successful, but it can be useful in alcoholism if supervised administration is available (Fuller 2004).

\subsubsection{Nicotine}

Nicotine replacement therapy is the most common form of smoking cessation therapy and comes in a number of different forms: nicotine patches, gums, lozenges, and nasal sprays. This therapy slowly releases nicotine into the body in an attempt to curb cravings. The main issue with this therapy is that nicotine is not thought to be the only addictive component in cigarettes, and therefore this therapy has low success rates of 7 $10 \%$ after a 1 year period (Balfour et al. 2000). Bupropion is a non-nicotine pharmacotherapeutic for smoking cessation and has a $14.6 \%$ success rate after 1 year (Jorenby et al. 2006). Buproprion is an atypical anti-depressant that is a non-selective 
DAT and norepinephrine transporter (NET) inhibitor and a partial nicotinic acetylcholine receptor (nAChR) inhibitor. It is unclear as to how bupropion helps patients quit smoking, but it is thought that the inhibition of DAT and NET help to alleviate withdrawal symptoms, and the inhibition of nAChR leads to decreased rewarding effects of cigarette smoking (Carroll et al. 2010). Varenicline is another non-nicotine smoking cessation therapy that has a success rate of $23 \%$ after 1 year (Jorenby et al. 2006). Varenicline is a partial nicotinic agonist and binds to the $\alpha 4 \beta 2$ nAChR to prevent nicotine from binding, but it still causes release of DA in the NAc, although to a lesser degree than nicotine. The increase in DA in the NAc is believed to cause a decrease in cravings by counteracting the decreased DA levels found during abstinence from nicotine (Coe et al. 2005).

\subsubsection{Opiates}

Opiate addiction is usually treated by a replacement or maintenance pharmacotherapy. This treatment involves replacement of the opiate being abused with another opiate that has a longer half-life and decreased abuse potential. As these therapies are also addictive, many patients continue taking these therapies rather than ceasing from all opiate use. The most commonly used replacement therapeutic is methadone. Methadone acts in a similar way to abused opiates and is addictive, but it has a longer half-life, which reduces cravings. It is administered either daily or every second day so that its use can be monitored and controlled (Corkery et al. 2004). This monitoring also helps to decrease the amount of methadone sold on the 'black market' to opiate abusers, decreasing the number of methadone-related deaths (Corkery et al. 2004). The use of methadone allows patients to integrate into normal society, increases the success of getting jobs and allows them to socialise more normally. Naltrexone can also be used to treat opiate addiction. Naltrexone is an opioid receptor antagonist and works by blocking the euphoric effects of the abused opiate. As with methadone, naltrexone treatment requires the patient to take supervised oral medication every day, which often leads to large drop-out rates in treatment programs (Hulse et al. 2009). Recently progress has been made on the use of a long-term, sustained release, naltrexone implant that increases success rates of opiate cessation as patients do not need to take supervised oral natrexone daily (Hulse et al. 2009). Buprenorphine is a more recently approved drug for opiate maintenance therapy that was first synthesised for use in pain relief. From the 1970's it has also been used as a treatment for opiate addiction and is successful in decreasing opiate use (Soeffing et al. 2009; Wesson and Smith 2010). As 
it is a partial mu-opioid receptor (MOPr) agonist and kappa-opioid receptor (KOPr) antagonist, it is thought to be safer to use than methadone, as it has similar efficacy and success and has less potential for overdose. Buprenorphine binds to the receptors to prevent opiates from binding and activates the receptors to a lesser extent then heroin or morphine (Wesson and Smith 2010).

\subsubsection{Amphetamines/Cocaine}

There is currently no FDA approved therapeutic available for cocaine, amphetamine, and methamphetamine addiction, although there are many studies investigating potential compounds. A large amount of research has gone into investigating the effects of DAT, NET, and SERT agonists for treatment of cocaine, amphetamine, and methamphetamine addiction. Positive results have been seen in animal studies and laboratory-based human studies, but there has been little success in clinical trials. Methylphenidate (Ritalin), modafinil (treatment for narcolepsy), and bupropion have been investigated for their effect on cocaine, methamphetamine, and amphetamine addiction in clinical trials with mixed results. Methylphenidate has shown promise as a potential therapeutic for amphetamine (Tiihonen et al. 2007), but had no effect on cocaine users. Modafinil has shown promise as a pharmacotherapeutic for drug abuse by decreasing cravings for cocaine and increasing the length of abstinence (Dackis et al. 2005; Dackis and O'Brien 2006). It also reduces methamphetamine use in patients who are only abusing methamphetamine and are undertaking counselling (Shearer et al. 2009). In 2004, a case study reported that self-administration of bupropion reduced cravings for amphetamine after seven days use, suggesting this drug may have promise as a therapeutic for amphetamine abuse (Tardieu et al. 2004). The overall data from these studies show promise for the successful development of a therapeutic that is able to treat cocaine, methamphetamine, and amphetamine addicts.

In summary, this section demonstrates that current available pharmacotherapies for treatment of drug addiction have low success rates and in the case of opiates are addictive themselves. There are also no current FDA approved therapeutics for amphetamine, cocaine, and methamphetamine abuse. Therefore, there is a need for investigation and trialling of new compounds with potential anti-addictive effects so that success rates for patients withdrawing from drug use can be increased. 


\subsection{Dopamine and the dopamine transporter}

\subsubsection{Introduction}

DA is a neurotransmitter that is found primarily in the brain and is involved in reward, motivation, motor function, decision making, compulsion, and stress (Mortensen and Amara 2003). DA is unable to cross the blood-brain barrier and is therefore synthesised within the brain from tyrosine by tyrosine hydroxylase to the dopamine precursor L-3,4dihydroxyphenylalanine (L-DOPA) that is then decarboxylated by DOPA carboxylase to DA (Flatmark 2000). DA is then packaged into vesicles for release into the synapse. Depolarisation of a DA neuron causes an increase in intracellular $\mathrm{Ca}^{2+}$, leading to release of DA by exocytosis. The DA then binds to postsynaptic receptors to continue the signal or to presynaptic receptors to regulate its release. DA is released in brain regions such as the NAc, PFC, striatum, and amygdala (Adinoff 2004).

The major regulator of DA concentration in the synapse is DAT. DAT is found perisynaptically on the presynaptic neuron and acts by taking DA back up into the synapse, where it is either recycled for further release, or degraded by MAO or COMT (Guo et al. 2007). The DA receptors, DRD1-5, also play a role in the regulation of DA concentration, in part by regulating DAT. These receptors are G protein-coupled receptors (GPCR) found on the pre- and post-synaptic membranes. The receptors are divided into two types, the DRD1-like and DRD2-like, with DRD1 and DRD5 receptors belonging to the DRD1-like group and DRD2, DRD3, and DRD4 belonging to the DRD2-like group. Both DRD2 and DRD3 act as DA autoreceptors on the presynaptic neuron, and via a feedback mechanism cause an increase in DAT function, and also a decrease in DA release. Dysregulation of the amount of DA found in the synapse leads to disorders such as Parkinson's disease and schizophrenia (Mehler-Wex et al. 2006).

All drugs of abuse either directly or indirectly cause increases in DA in the synapse, either through direct interactions with DAT or by interactions with inhibitory neurons that communicate with dopaminergic neurons. This leads to prolonged activation of pre- and post-synaptic receptors that increase the strength of the signalling pathways. After repeated drug taking, the dopaminergic pathway is desensitised, leading to a state of allostasis, as described in section 1.1.4. 


\subsubsection{The dopamine transporter}

The dopamine transporter is a $\mathrm{Na}^{+} / \mathrm{Cl}^{-}$symporter protein from the gene family SLC6. Other transporters belonging to this family include SERT, NET, and transporters for $\gamma$ aminohydroxybutyric acid (GABA), glycine, amino acids, creatine, and organic osmolytes (Howell and Kimmel 2008). The members of this family are characterised structurally by their 12 transmembrane domains, large extracellular loop 2 that contains glycosylation sites, and intracellular $\mathrm{C}$ and $\mathrm{N}$ termini (Figure 1.3). The structure of the $\mathrm{Na}^{+} / \mathrm{Cl}^{-}$symporter protein family has been estimated using the bacterial homologue leucine transporter LeuT $_{\text {Aa }}$ from the bacteria Aquifex aoelicus (Yamashita et al. 2005). While this bacterial transporter has a low sequence homology to mammalian transporters, it shows high protein sequence homology, and the binding site of LeuT $\mathrm{T}_{\mathrm{Aa}}$ shows high sequence homology with DAT. Experiments using LeuT $\mathrm{T}_{\mathrm{Aa}}$ have been carried out to identify the binding site of leucine and the $2 \mathrm{Na}^{+}$ions that are cotransported, and models of how transport occurs have also been theorised for both LeuT $_{\text {Aa }}$ and the catecholamine transporters (Yamashita et al. 2005; Beuming et al. 2006; Wang and Lewis 2009). Based on the LeuT $\mathrm{Aa}_{\mathrm{Aa}}$ structure, the binding position of the substrate and $\mathrm{Cl}^{-}$(Forrest et al. 2007), the selectivity of the $\mathrm{Na}^{+}$ions (Yamashita et al. 2005) and the transport and release of $\mathrm{Na}^{+}$and substrates inside the cell of monoamine transporters have been modelled (Yamashita et al. 2005; Shi et al. 2008b).

DAT is found selectively expressed in neurons located in the VTA and substantia nigra (Ciliax et al. 1995), and DAT protein is found in very high amounts in the dorsal striatum and NAc where it is involved in reward (Boja and Kuhar 1989; Scheffel et al. 1991). A study carried out looking at dopaminergic neurons that innervate the NAc found that DAT protein is expressed on the axons, dendrites or cell body of the cells, but not in the synapse (Nirenberg et al. 1997).

During tonic DA firing periods, DAT can also act as an ion channel, which causes an increase in neuronal depolarisation leading to an increase in exocytotic DA release. During phasic firing, this role is over-ridden by the presynaptic DRD2 receptors that decrease DA release (Sonders et al. 1997; Elliot and Beveridge 2005).

The rat (Kilty et al. 1991; Shimada et al. 1991) and human DAT (Giros et al. 1992) genes were first cloned and expressed in cell systems in the early 1990's. This allowed more detailed research to take place on the structure, mechanism, and pharmacology of 
DAT, as cell lines that do not usually express DAT could be transfected and easily manipulated. Interestingly, DAT appears to function differently in cell models compared to rat tissue. This could be due either to differences in the human DAT and rat DAT (Giros et al. 1992) or could be because of differences in the native and nonnative expression systems (McElvain and Schenk 1992; Povlock and Schenk 1997; Chen et al. 1999b; Earles and Schenk 1999b). Studies using human embryonic kidney (HEK-293) cells transfected with the human DAT showed that $2 \mathrm{Na}^{+}$ions bound to DAT, followed by DA, then $1 \mathrm{Cl}^{-}$ion before transport occurs (Chen et al. 1999b; Earles and Schenk 1999b). Other studies using isolated rat striatal (McElvain and Schenk 1992) and nucleus accumbens (Povlock and Schenk 1997) tissue found that binding of DAT substrates was partially random, with $2 \mathrm{Na}^{+}$or $1 \mathrm{DA}$ binding, followed by $\mathrm{Cl}^{-}$, before DA transport occurred.

All drugs of abuse either directly or indirectly lead to an increase in DA in the synapse. Cocaine acts by directly binding to DAT, preventing uptake of DA into the presynaptic neuron (Figure 1.2). This leads to accumulation of DA in the synapse, causing increased activation of the DA receptors. Amphetamine-like drugs such as amphetamine and methamphetamine act as substrates for DAT and are taken up into the cell where they are transported by vesicular monoamine transporters (VMAT) into vesicles. Here they cause transport of DA out of vesicles by VMAT-2 and then reverse transport of cytosolic DA through DAT, leading to increases in DA in the synapse. Other drugs of abuse such as nicotine, alcohol, and morphine work in a more indirect fashion. In the NAc, nicotine binds to nAChRs found in dopaminergic cell bodies, and this leads to release of DA into the synapse. Opioids bind to opioid receptors which in turn inhibit GABA neurons that usually act to inhibit the dopaminergic neurons. This leads to dis-inhibition of dopaminergic neurons and release of DA into the synapse. Alcohol is also thought to act through a similar pathway as the opioids and has been shown to cause release of opioid peptides.

\subsubsection{Regulation of the dopamine transporter}

Regulation of DAT occurs through many different pathways, and most regulation has been linked to changes in cell surface expression of DAT. Constitutive DAT exocytosis and endocytosis occur, and this is thought to be mediated through proteins including Rab proteins, which are involved in targeting proteins to recycling and degradation 
pathways (Furman et al. 2009b). Some of the major regulators of DAT function are outlined below.

\subsubsection{Dopamine}

DA regulates DAT cell surface expression, with short-term exposure leading to an increase in DAT cell surface expression, and repeated and long-term exposures causing a decrease in cell surface expression. In HEK-293 cells, $10 \mu \mathrm{M}$ DA results in an immediate increase in DAT cell surface expression, with the maximal effect seen at 1 min using total internal reflection fluorescence microscopy (TIRFM) and cell surface biotinylation (Furman et al. 2009a). Longer incubations of between 40 and 60 min of single exposures to 10-100 $\mu \mathrm{M}$ DA, showed a decrease in DAT cell surface expression in both cell systems and in rat striatal synaptosomes (Saunders et al. 2000; Chi and Reith 2003). Short repeated applications of DA to Xenopus oocytes also showed decreases in [3H]-DA uptake and DAT cell surface expression (Gulley et al. 2002). In vivo application of DA into the rat striatum also caused a large decrease in DA uptake, as measured by high speed chronoamperometry, suggesting decreased DAT cell surface expression (Gulley et al. 2002). Interestingly, this effect was not seen in the NAc. These short- and long-term effects were both mediated by protein kinase C (PKC) dependent pathways (Gulley et al. 2002; Furman et al. 2009a). This decrease in cell surface expression of DAT is thought to be protective, since high concentrations of DA within the cell would be toxic and lead to cell death.

\subsubsection{Dopamine receptors}

DRD2 and DRD3 are pertussis toxin (PTX) sensitive GPCRs found on dopaminergic neurons (Dal Toso et al. 1989; Diaz et al. 2000). These receptors both belong to the DRD2-like DA receptor family and have both been shown to modulate DA levels in the synapse by decreasing DA synthesis and release (Tang et al. 1994; O'Hara et al. 1996) and modulating DAT function and cell surface expression (Bolan et al. 2007; Zapata et al. 2007). This forms a feedback mechanism, allowing DA to regulate its own extracellular levels.

DRD2 has been found both pre-synaptically and post-synaptically on dopaminergic neurons (Lindgren et al. 2003). The receptor is found in two isoforms, long (DRD2L) and short (DRD2S), which differ in length by alternative mRNA splicing of a 29 amino acid segment in the third intracellular loop (Dal Toso et al. 1989). Activation of DRD2 
by the DRD2/D3 agonist quinpirole leads to increased DAT function in rat striatal tissue (Meiergerd et al. 1993) and in HEK-293 cells co-transfected with DAT and DRD2 (Bolan et al. 2007). This increase in function was found to occur through an extracellular-signal regulated kinase 1/2 (ERK1/2) and Akt pathway, and was ERK1/2 dependent. The increase in function was mirrored by rapid increases in cell surface expression, and these changes were hypothesised to be due to a direct protein-protein interaction between DAT and DRD2 (Bolan et al. 2007). This was confirmed in another study that used co-transfected HEK-293 cells and rat striatal tissue to show that DRD2 and DAT were directly interacting through a protein-protein interaction, which caused recruitment of DAT to the plasma membrane, leading to increased DA uptake (Lee et al. 2007).

The DRD3 receptor is more restricted in its distribution than DRD2, but has also been identified as a DA autoreceptor (Levesque et al. 1992). Studies using fast-scan cyclic voltammetry and DRD2 and DRD3 agonists in the mouse have shown that DRD3 receptors have a hierarchical activity in the NAc shell, followed by less activity in the core and the lowest activity in the striatum. In contrast, DRD2 receptors were found to have a high activity in all three brain regions (Maina and Matthews 2010). Knockout mouse studies have shown that DRD3 regulates extracellular DA levels, but the mechanisms underlying this effect were not elucidated (Zapata et al. 2001; Joseph et al. 2002). Further studies found that activation of DRD3 with the DRD3 agonist (+)PD128907 led to decreases in extracellular DA in mouse NAc and an increase in DAT function in rat NAc tissue (Zapata and Shippenberg 2002). Antagonism of DRD3 caused a decrease in DAT function in rat NAc tissue (Zapata and Shippenberg 2002) confirming that this affect was mediated by DRD3. A further study was conducted in HEK-293 cells co-expressing DRD3 and DAT (Zapata et al. 2007). Short-term addition of the DRD2/3 agonist quinpirole caused an increase in DAT function that was mediated through ERK1/2 and PI3K phosphorylation pathways and led to increases in DAT cell surface expression (Zapata et al. 2007). Long-term incubation with quinpirole led to the opposite effect, with a decrease in DAT function and cell surface expression (Zapata et al. 2007).

Overall, these studies demonstrate the close regulation that DA in the synapse has on DA autoreceptors and DAT function, and how important the regulation of DA in the synapse is for brain homeostasis. 


\subsubsection{Phosphorylation}

DAT function and cell surface expression has been shown to be altered by its phosphorylation states. DAT has several ser/thr residues present in the $\mathrm{N}$-terminal tail that are involved in DAT phosphorylation and regulation (Figure 1.3). These ser/thr residues can be phosphorylated by a number of different kinases as follows.

PKC is activated endogenously by diacylglycerol (DAG) and can be pharmacologically activated by phorbol esters such as phorbol 12-myristate 13-acetate (PMA). Activation of PKC leads to a decreased DAT Vmax, without altering $\mathrm{Km}$, due to internalisation of DAT in cell models and rat striatal synaptosomes (Copeland et al. 1996; Vaughan et al. 1997; Pristupa et al. 1998). A study carried out in LLC-PK ${ }_{1}$ cells found that after $4 \mathrm{~min}$ incubation with PMA, there was an increase in phosphorylation of DAT and a decrease in Vmax (Huff et al. 1997); however, other studies have shown that DAT is still internalised after PMA treatment when DAT ser/thr phosphorylation sites have been mutated (Granas et al. 2003). Therefore, it is uncertain whether internalisation of DAT by PKC occurs by direct phosphorylation or if it is due to phosphorylation of other proteins that complex with DAT. PKC-induced DAT endocytosis has been shown to be dynamin dependent (Daniels and Amara 1999). It is unknown whether the internalised DAT is recycled or degraded, with reports showing evidence for both mechanisms (Daniels and Amara 1999; Melikian and Buckley 1999). Differences in these reports may be due to the different cell models used and their differences in basal cell surface expression of DAT.

Protein Kinase A (PKA) is activated by cyclic adenosine monophosphate (cAMP) in the cell. This is determined by adenylyl cyclase, which is regulated by receptors such as the DRD2. PKA activation increases DAT Vmax but not $\mathrm{Km}$ in rat stratial tissue, with increases seen from between 1 and 15 min (Batchelor and Schenk 1998). An increase in DAT cell surface expression after PKA activation was also seen in a cell model (Pristupa et al. 1998). Other studies have not seen changes in DAT expression with PKA activation, but this may be due to the time points they investigated being too long and therefore causing the effect to be missed (Copeland et al. 1996; Pristupa et al. 1998). In rat striatal synaptosomes, addition of 8-bromo-cAMP caused an increase in DAT function, and inhibition of PKA attenuated this effect, indicating that in this system PKA activation leads to increased DAT function (Page et al. 2004). 
There are four major ser/thr protein phosphatases (PPs): PP1, PP2A, PP2B and PP2C. These phosphatases can be separately studied as they are inhibited by specific compounds. PP1 and PP2A are strongly inhibited by okadaic acid, microscystin inhibits PP1 and PP2A, calcyculin A inhibits PP2A twice as strongly as PP1 and cyclosporin A selectively inhibits PP2B (Vaughan et al. 1997). In rat straital synaptosomes, okadaic acid and calcyculin both increase phosphorylation of DAT and reduce DAT function; whereas, cyclosporin A and microcystin have no effect (Vaughan et al. 1997). This suggests that PP2B is involved in DAT phosphorylation and downregulation in synaptosomes, and the authors postulated that cylcosporin A has no effect due to its poor membrane solubility.

There is also some evidence that protein tyrosine kinases modulate DAT function and cell surface expression (Hoover et al. 2007). Inhibition of tyrosine kinases by genistein and tyrphostin 23 in rat striatal synaptosomes, primary rat mesencephalic cultures, and Xenopus oocytes leads to rapid decreases in Vmax but not $\mathrm{Km}$ of DAT and causes a decrease in cell surface expression of DAT (Doolen and Zahniser 2001; Hoover et al. 2007). In primary rat mesencephalic cultures the addition of brain-derived neurotrophic factor also leads to an increase in DAT function (Hoover et al. 2007).

$\mathrm{Ca}^{2+} /$ calmodulin-dependent kinase II (CamKII) also appears to modulate the function of DAT (Page et al. 2004). In rat striatal synaptosomes, the addition of a CamKII inhibitor, KN62, inhibited the increase in transporter function caused by 8-bromocAMP (Page et al. 2004). The CamKII inhibitor alone did not cause changes in DAT function, indicating that this process occurs through a cAMP dependent pathway.

Mitogen-activated protein kinases (MAPKs) are ser/thr kinases that are involved in the regulation cell growth and differentiation (Moron et al. 2003) and are found throughout the central nervous system. There are at least 12 different MAPKs, including ERK1/2, c-Jun N-terminal kinase (JNK1-3) and p38 $(\alpha \beta \gamma \delta)$ stress kinases (Bruchas and Chavkin 2010). MAPKs are activated through several mechanisms, including GPCRs, calcium and growth factors, and they can be activated by PKC-dependent or independent mechanisms. The inhibition of MAPK in EM4 cells and rat straital synaptosomes caused a concentration and time dependent down-regulation of DA uptake that was found to be due to a decrease in cell surface expression of DAT (Moron et al. 2003). 
This effect was seen with ERK1/2 inhibitors, but not with p38MAPK inhibitors, indicating that p38 MAPK is not involved in DAT regulation.

To conclude, activation of many different phosphorylation pathways leads to modulation of DAT function, mostly caused by increased or decreased cell surface expression of DAT. When activated, PKC and PP2B cause down-regulation of DAT; whereas, activation of PKA, tyrosine kinases, CaMKII and the ERK1/2 pathway all lead to increased DAT function and cell surface expression. This highlights the tight regulation that DAT is under in the brain, and indicates that many different receptors and pathways have the ability to modulate its function.

\subsubsection{Glycosylation}

DAT proteins are post-translationally glycosylated on asparagines found on its extracellular loop 2. Rat and human glycosylation sites are different (Figure 1.3) with four glycosylation sites for rat and three for human (Patel et al. 1993). There have been several studies carried out to investigate if $N$-glycosylation of DAT is necessary for cell surface expression and function (Torres et al. 2003; Li et al. 2004b). Two studies mutated the three glycosylation sites on human DAT and expressed the single mutants, double mutants and triple mutants in HEK-293 cells. While their results differed, both groups showed that glycosylation was important for DAT function, but not crucial for insertion into the cell membrane. Removal of $N$-glycosylation sites; however, led to a decrease in cell surface expression of DAT. Reasons for this may be that nonglycosylated DAT is vulnerable to degradation or less stably expressed on the cell surface (Li et al. 2004b).

\subsubsection{Ubiquitination}

Ubiquitination of DAT has also been shown to cause internalisation of DAT. This is mediated by PKC activation (Miranda et al. 2005) and has been shown to involve Nedd4.2, an E3 ubiquitin ligase and the ubiquitin-conjugating enzymes ube2d2/3 and ube213 e2 (Vina-Vilaseca and Sorkin 2010). Studies have found that DAT is polyubiquitinated on the amino terminal end on Lys 19, 27, and 35, leading to internalisation of DAT (Figure 1.3) (Miranda et al. 2007). Ubiquitination of either one or two of these lysines does not cause internalisation as shown by DAT mutant studies (Miranda et al. 2007). Ubiqutination of DAT forms a DAT which is $26 \mathrm{kDA}$ larger then mature DAT, suggesting 4 ubiquitination sites (Miranda et al. 2005; Vina-Vilaseca and Sorkin 2010). 
Ubiquitination of DAT has been shown to be important for sorting of internalised DAT proteins to recycling endosomes and lysosomes (Miranda et al. 2007).

\subsubsection{Drugs of abuse and dopamine signalling}

All drugs of abuse directly or indirectly lead to changes in DA signalling in the brain. The effects of amphetamine, cocaine, nicotine, alcohol, and opiates on DAT function and expression are outlined below.

\section{Amphetamine}

Amphetamine is a substrate for DAT and shows a biphasic effect on DAT, with the immediate effects being a rapid, short-lived increase in DAT cell surface expression of 1 to 2 min (Furman et al. 2009a). Repeated or long-term exposure leads to a decrease in function and cell surface expression (Gulley et al. 2002; Richards and Zahniser 2009). This down-regulation is dependent on the PKC pathway (Gulley et al. 2002) In vitro experiments carried out in Xenopus oocytes were carried out in which repeated short exposures to amphetamine led to decreased DAT function, and this decrease was inhibited by a PKC inhibitor (Gulley et al. 2002). A decrease in function was also observed using in vivo electrochemistry in the rat dorsal striatum after repeated administration of amphetamine, but was not seen in the NAc (Gulley et al. 2002). A later study investigated the apparent difference between the NAc and striatum with regard to the effects of amphetamine in synaptosomes prepared from these brain regions. In the striatum, a decrease in Vmax was found with no change in $\mathrm{Km}$ in synaptosomes treated with $20 \mu \mathrm{M}$ amphetamine. No effect was seen in the NAc. In this same study, synaptosomes prepared from rats exposed to amphetamine $(2 \mathrm{mg} / \mathrm{kg}) 45$ min before euthanasia showed decreases in Vmax in both brain regions. These changes were PKC dependent, but there were no changes in cell surface expression of DAT when the cells were exposed to amphetamine for less than $1 \mathrm{~h}$ (Richards and Zahniser 2009). Experiments carried out in HEK-293 cells expressing FLAG-hDAT found that internalisation was also dynamin dependent, suggesting that it occurred via clatherincoated pit mediated endocytosis (Saunders et al. 2000).

Amphetamine $(10 \mu \mathrm{M})$ applied to EM4 cells expressing YFP-hDAT also caused an immediate reverse transport of DA through DAT, increasing the synaptic concentration of DA. This is thought to occur in two main ways: a slow, transport-like mechanism, and a fast, channel-like mechanism (Kahlig et al. 2005). In the slow transport-like 
mechanism, amphetamine is a substrate for DAT and is taken up, increasing the number of 'inward' facing transporters and leading to increased intracellular DA reverse transport. In the channel-like mechanism, DAT has channel-like bursts of DA release. This process has been shown to be dependent on phosphorylation at the N-terminus of DAT, specifically at ser7 and ser12 which appear to be phosphorylated by PKC and MAPK kinase 1/2 (MEK1/2) (Khoshbouei et al. 2004). In rat striatal slices, PKC $\beta$ was shown to physically interact with DAT and was necessary for amphetamine-induced efflux (Johnson et al. 2005). Reverse transport has also been shown to be CamKIIa dependent, with CAMKIIa binding directly to DAT in dopaminergic neurons and in EM4 cells expressing DAT (Fog et al. 2006). The SNARE protein syntaxin 1A also binds DAT, leading to CAMKII-dependent amphetamine-induced efflux (Binda et al. 2008).

\section{Cocaine}

Cocaine acts as a DAT inhibitor, by binding to DAT and preventing DA uptake. Cocaine interacts with similar amino acids on DAT as DA but does not bind in the exact same place (Huang et al. 2009b) (Figure 1.3). There are conflicting data on whether cocaine inhibits DA uptake in a competitive (Wu et al. 2001), uncompetitive (McElvain and Schenk 1992), or non-competitive (Povlock and Schenk 1997) mechanism, and these differences have been proposed to be dependent on the experimental technique used (Povlock and Schenk 1997). There have also been conflicting data on how cocaine regulates DAT, i.e. whether there are increases or decreases in function and the nature of the effect on cell surface expression. This is thought to be because of the different treatment schemes and models used to investigate cocaine's effects, and the different brain regions investigated. A study was carried out in Rhesus monkeys given either low $(0.03 \mathrm{mg} / \mathrm{kg} /$ injection $)$ or high dose $(0.3 \mathrm{mg} / \mathrm{kg} /$ injection $)$ cocaine for 5 days or 3.3 months (Letchworth et al. 2001). Some monkeys were also self-administering cocaine ( $0.03 \mathrm{mg} / \mathrm{kg} / \mathrm{injection})$ for 1.5 years. The results showed that after the initial 5-day period, there was decreased $\left[{ }^{3} \mathrm{H}\right] \mathrm{WIN} 35,428$ binding in the striatal regions of the brain, but after 3.3 months and 1.5 years there were increases in $\left[{ }^{3} \mathrm{H}\right] \mathrm{WIN} 35,428$ binding in these brain regions. This suggests a decrease in DAT during short treatments, with longterm treatments causing increased DAT protein. A study carried out in post-mortem human cocaine users confirmed the increase in binding after long-term cocaine use, with an increase in $\left[{ }^{3} \mathrm{H}\right]$ WIN35,428 binding to DAT and an increase in DAT function in the striatum (Mash et al. 2002). This effect was not seen in patients that had excited 
cocaine delirium and who had paranoia and agitation before being admitted to hospital. This increase was proposed to be a feedback mechanism from long-term cocaine use, with an increase in cell surface DAT to counteract the increase in DA due to cocaine use, decreasing the rewarding effect and leading to increased drug use (Mash et al. 2002). In cocaine-dependent subjects, single-photon emission computed tomography (SPECT) studies have also shown an increase in DAT density in the caudate putamen region (Crits-Christoph et al. 2008).

Cell model studies using cells transfected with human DAT show that cocaine causes an increase in DAT cell surface expression after acute (10 $\mu \mathrm{M}, 10 \mathrm{~min}$; (Daws et al. 2002) and chronic (1 $\mu \mathrm{M}, 24 \mathrm{~h}$, (Zhang et al. 1998); $1 \mu \mathrm{M} 12 \mathrm{~h}$, (Little et al. 2002)) treatment, and an increase in DA uptake (Zhang et al. 1998; Daws et al. 2002; Little et al. 2002). This effect appeared to be cell specific as these changes were seen with EM4 cells and N2A cells, but not COS7 cells (Zhang et al. 1998). Experiments carried out after early abstinence in rats also show increased DAT function (Thompson et al. 2000), but there are conflicting data as some reports show down-regulation or no change in uptake during early abstinence (Chefer and Shippenberg 2002). These differences are thought to be due to experimental design, such as the amount of cocaine given/selfadministered, the length of exposure time to cocaine, and differences in withdrawal times. There is also evidence that cocaine causes release of DA from reserve pools of DA-containing synaptic vesicles in a synapsin dependent manner (Venton et al. 2006). This would further add to the DA in the synapse, increasing the reward signal.

\section{Nicotine}

Nicotine indirectly acts on the DA system through nAChRs. There are much conflicting data on how nicotine modulates the dopaminergic system with reports suggesting up- and down-regulation of DAT. Acute nicotine administration of 0.8 $\mathrm{mg} / \mathrm{kg}$ causes an increase in $\left[{ }^{3} \mathrm{H}\right]$-DA uptake and cell surface expression of DAT in the PFC but not the striatum, with a lower dose $(0.3 \mathrm{mg} / \mathrm{kg}$ ) having no effect (Zhu et al. 2009). Another study found that after 5, 10, and 40 min there was an increase in $\left[{ }^{3} \mathrm{H}\right]$ DA uptake in the striatum with acute $0.32 \mathrm{mg} / \mathrm{kg}$ injections of nicotine and no change in cell surface expression (Middleton et al. 2007). Furthermore, in vivo voltammetry measurements showed increased DA clearance in the striatum and medial PFC of rats acutely treated with nicotine for 10 to $15 \mathrm{~min}$ (Middleton et al. 2004). In contrast, SPECT studies in male smokers found that there was a decrease in DAT availability in 
the striatum, with no changes in DRD2 or DRD3 receptors (Yang et al. 2008). Chronic exposure to cigarette smoke and nicotine also increases DAT mRNA in the rat midbrain areas: the VTA and substantia nigra ( $\mathrm{Li}$ et al. 2004c). This was also observed in the human neuroblastoma cell line, SK-N-SH, transfected with human DAT in which nicotine caused an increase in transcription of human DAT, and this effect was neuronal cell line specific (Ohyama et al. 2010). There are differences between studies carried out in brains of animals treated with nicotine and in cell systems, which suggest whole brain circuitry, or other unknown factors found within the brain cells, are required for these effects to be properly represented in a cell model.

\section{Alcohol}

Alcohol also indirectly acts on the dopaminergic system through inhibition of NMDA receptors and activation of GABA $\alpha$ receptors, although there is evidence that alcohol can directly stimulate dopaminergic neurons in the VTA causing DA release (Brodie et al. 1999). Inconsistent results have been gained about the effect of alcohol on DAT, with animal studies suggesting that acute treatment with alcohol leads to increases (Wang et al. 1997), decreases (Lin and Chai 1995; Robinson et al. 2005) or no change (Yim and Gonzales 2000; Budygin et al. 2001) in DAT function in the striatum and NAc. These differences in results may be due to the varied procedures carried out, such as microdialysis, fast scan cyclic voltammetry, in vivo electrochemical detection, rotating disk electrode voltammetry and use of awake and anesthetised rats. However the majority of acute studies suggest that ethanol increases extracellular DA in the NAc and striatum due to increased release (Yim et al. 1998; Yim and Gonzales 2000; Budygin et al. 2001; Robinson et al. 2005). Human alcoholic studies have shown that there is a decrease in DRD2 receptors in current alcoholics but not a decrease in DAT (Volkow et al. 1996). Using SPECT studies, alcoholics admitted for detoxification showed decreased DAT, but after 4 weeks of abstinence a significant increase in DAT was seen compared to healthy controls (Laine et al. 1999). This implies that changes in the dopaminergic system vary depending on the length of time since consumption of alcohol. Chronic animal studies have also shown decreases in DRD2 receptors and DAT in the NAc of rats exposed to $10 \%$ ethanol as their only water source for 30 days (Yoshimoto et al. 2000). In rats fed a liquid diet for 1 year in which $36 \%$ of their source of calories was alcohol, there was a significant increase in DAT in the NAc and striatum and a decrease in tissue DA levels (Rothblat et al. 2001). These studies differ in the 
length of time the animals were exposed to alcohol, and this may explain the differences in data.

Data collected from cell models show more consistency. Most studies have found that alcohol leads to an increase in DAT function, and this is due to increased cell surface expression of DAT (Mayfield et al. 2001; Maiya et al. 2002). The differences between cell and animal studies are most probably due to the fact that alcohol targets more than one pathway in the brain, and many of its effects are due to interactions between brain regions. As cell systems are more simplistic they are often unable to replicate the effects seen in animal studies where intra-regional interactions may play a role.

\section{Opioids}

Opioids also indirectly affect DA in the mesolimbic dopaminergic pathway. They act on the inhibitory GABA neurons, dis-inhibiting their effect on dopaminergic neurons and leading to increases in synaptic DA in the NAc (Di Chiara and Imperato 1988a; Klitenick et al. 1992).

\section{Morphine}

Chronic, but not acute, morphine administration leads to decreased DAT in the NAc of rats (Simantov 1993). DAT binding was measured by SPECT in Rhesus monkeys treated with increasing morphine doses $(0.5,1.6,5.0,8.0,8.0$, and $8.0 \mathrm{mg} / \mathrm{kg}$ for each successive day) twice daily for six days. After the initial morphine injection, there was a sharp decrease in DAT binding in the striatum that slowly increased over five days following morphine administration (Xiao et al. 2006). After the five days, DAT binding in the striatum was still lower then controls, suggesting that there was a semi-permanent change in DAT expression after morphine treatment. A study carried out by Gudehithlu and Bhargava (1996) in morphine tolerant (rats who had six $75 \mathrm{mg}$ morphine pellets implanted for 7 days) and withdrawn rats (rats treated with six $75 \mathrm{mg}$ morphine pellets for 7 days, which were removed $16 \mathrm{~h}$ before euthanasia), it found that there was increased DAT in the hypothalamus in both the tolerant and withdrawn rats. In tolerant rats a decrease of DAT was seen in the striatum and spinal cord. A decrease in hippocampal DAT was also seen following morphine withdrawal. The authors speculated that these differences might contribute to abstinence syndrome (Gudehithlu and Bhargava 1996). 


\section{Heroin}

In positron emission tomography (PET) studies carried out on human heroin users following long term abstinence without pharmacotherapy (drug use for at least 3 years, free of illicit drugs for 6 months) or following treatment with methadone, a significant decrease in DAT function was seen in the bilateral caudate compared to drug naïve controls. The patients on methadone also had a significantly lower DAT function in the putamen compared to controls and abstinent heroin users (Shi et al. 2008a). In postmortem human studies, chronic heroin use caused decreased mRNA expression in the paranigral neurons and VTA and a decrease in DAT protein in the NAc (Kish et al. 2001; Horvath et al. 2007). These studies demonstrate that after chronic heroin use there is a significant decrease in DAT within the brain, similar to the effects of morphine, and that this effect is still seen after 6 months.

Overall these studies indicate that drugs of abuse lead to changes in the dopaminergic system, with most drugs of abuse causing an acute increase in DAT function or expression in response to the increased DA available in the synapse. Over a long period of drug taking or exposure, however, there appears to be a general decrease in DAT in the striatum and NAc that may continue after drug taking has ceased. This decrease in DAT may contribute to an individual's susceptibility to relapse to drug taking due to the muted dopaminergic transmission in the reward pathway. 


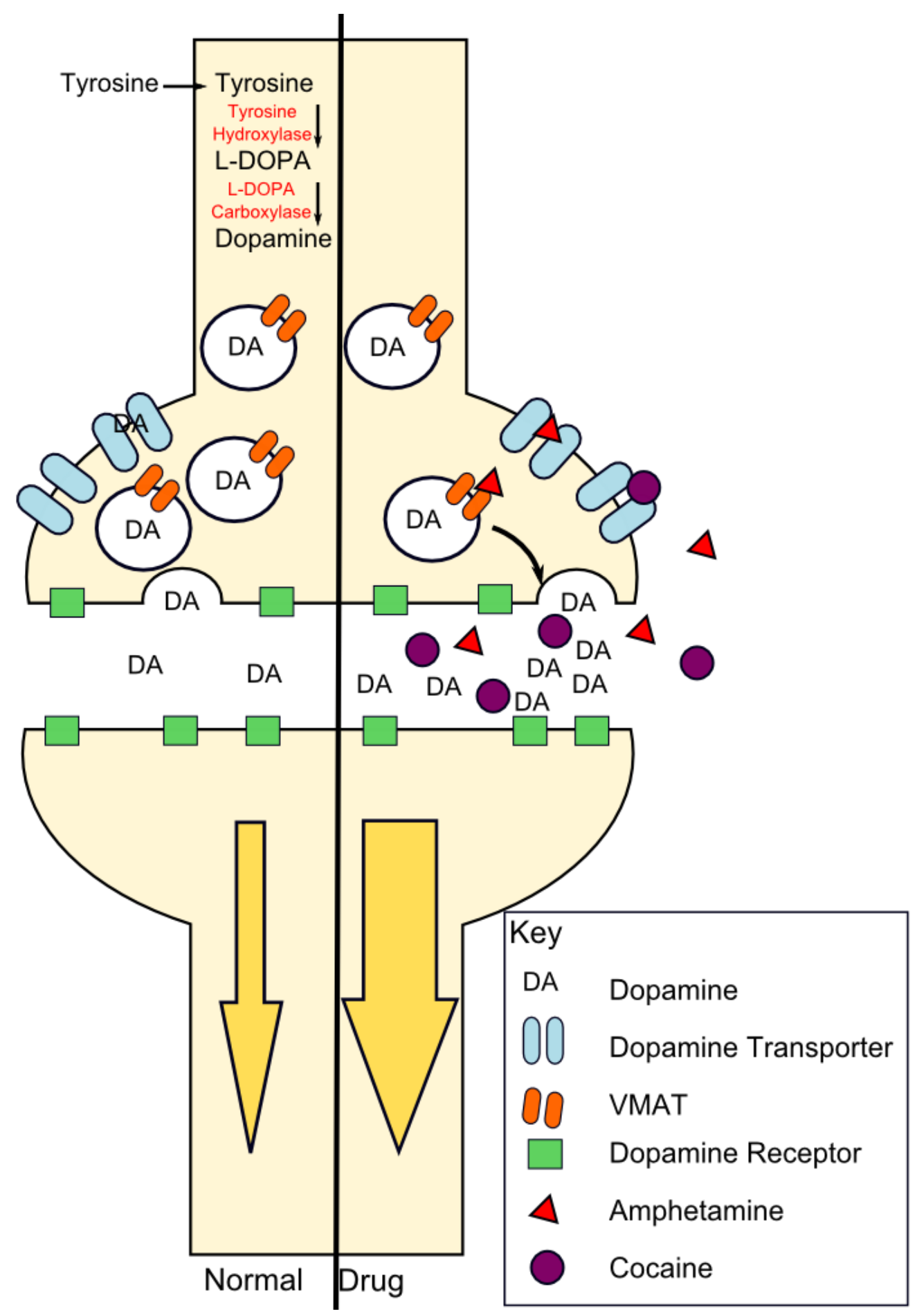

Figure 1.2: Dopamine at the synapse. DA is synthesised in the cell body from tyrosine where it is packaged into vesicles for release at the synapse. At depolarisation of the neuron, an increase in $\mathrm{Ca}^{+2}$ occurs, and dopamine is released by exocytosis into the synapse. In the synapse, DA binds to post-synaptic receptors which cause depolarisation in the post-synaptic neuron continuing on the signal. DAT are found perisynaptically and act to take DA back into the pre-synaptic neuron, attenuating the signalling. Cocaine binds to DAT and blocks its function, leading to an increase in DA in the synapse causing increased signalling. Amphetamine acts as a substrate of DAT and is taken up into the cell where it interacts with VMAT, causing large-scale release of DA into the synapse by reverse transport and exocytosis. This also causes an increase in DA in the synapse leading to increased signalling. 


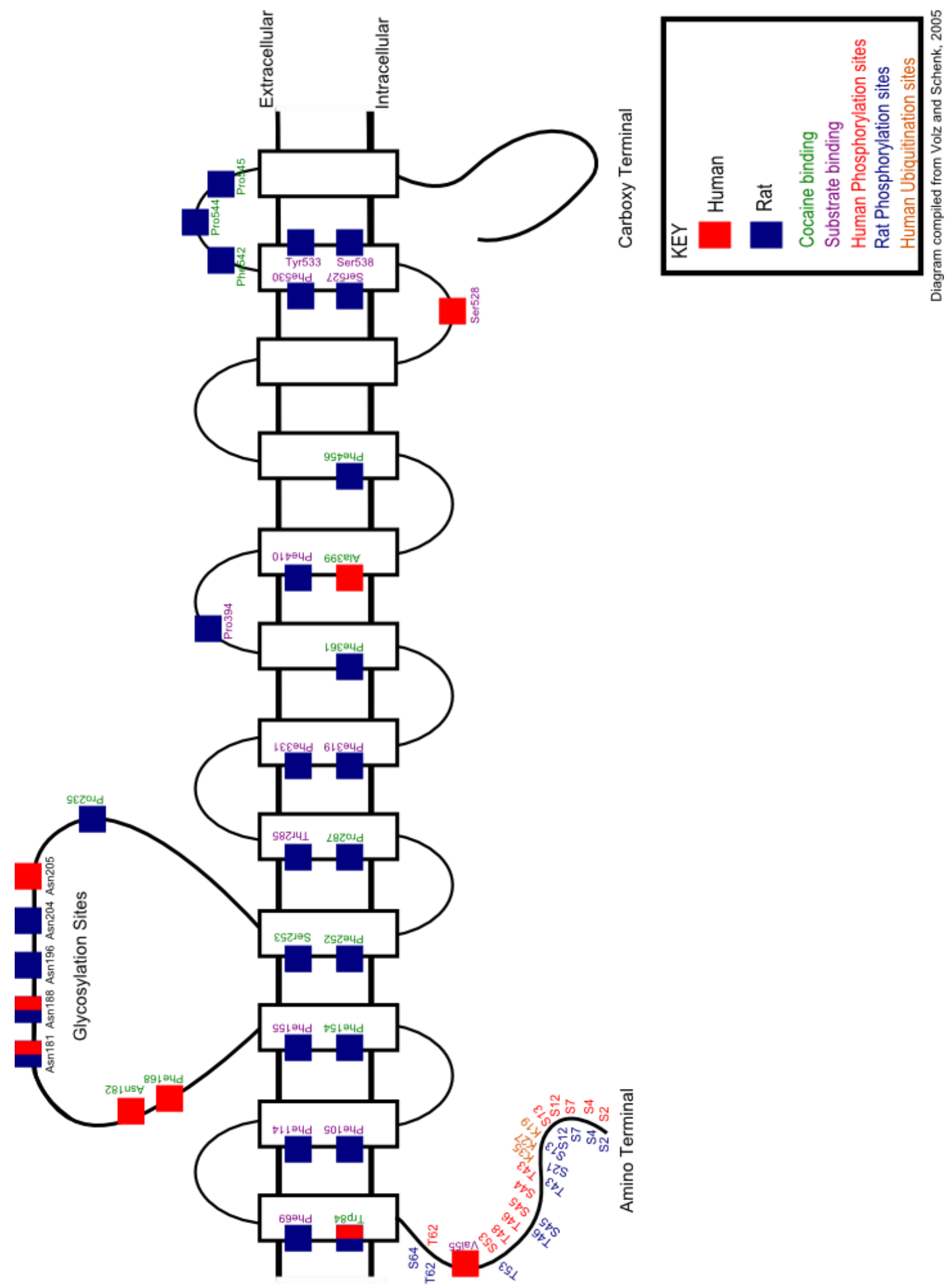

Figure 1.3: Important glycosylation, phosphorylation, ubiquitination and binding sites for DA and cocaine on the rat and human DAT. Ala - alanine, Asn asparagine Phe - phenylalanine, Pro - proline, S- serine, T, Thr - threonine, Val valine. Compiled from Volz and Schenk, 2005 (Volz and Schenk 2005) 


\subsection{The Serotonin Transporter}

Serotonin (5-HT) is a major brain neurotransmitter involved in many pathways, including mood, memory, reward, and sleep (Murphy et al. 2008). The release of 5-HT into synapses leads to the activation of pre- and post-synaptic receptors, and attenuation of this signalling is achieved through reuptake of 5-HT by SERT. SERT belongs to the $\mathrm{Na}^{+} / \mathrm{Cl}^{-}$dependent transporter gene family SLC6, along with the transporters for other neurotransmitters such as DA, norepinephrine, and amino acids such as glycine and GABA (Hoffman et al. 1998). In the brain SERT is a target for drugs of abuse such as MDMA and cocaine, which cause an acute large-scale increase of 5-HT in the synapse. This increase results in amplification of 5-HT signalling and activation of the brain's reward pathways (Muller et al. 2003). SERT has been implicated in a number of disorders including depression (Goldman et al. 2010), anxiety (Laucht et al. 2009), and substance abuse (Ramamoorthy et al. 1993), and is a pharmacological target for therapeutics such as the SSRIs (Jones and Blackburn 2002; Murphy et al. 2008). Due to the behavioural significance of 5-HT tone in the brain, SERT activity is central to the understanding of many psychological disorders, and is a target for the development of novel therapeutics.

SERT is found on the peri- and extra-synaptic regions as well as the axon and axon varicosities of serotinergic neurons in the striatum, NAc, hippocampus, substantia nigra, and PFC (Pickel and Chan 1996; Sur et al. 1996; Miner et al. 2000). Uptake of 5-HT by SERT is dependent on the co-transport of $\mathrm{Na}^{+}$and $\mathrm{Cl}^{-}$ions in a 1:1:1 ratio (Ramamoorthy et al. 1993; Chang and Lam 1998). It has been proposed that $\mathrm{Cl}^{-}$is crucial for 5-HT binding to SERT, and that $\mathrm{Na}^{+}$is necessary for 5-HT transport to occur (Chang and Lam 1998). When $\mathrm{Na}^{+}$is replaced with ions such as choline (Blakely et al. 1991), lithium (Gu et al. 1994; Chang and Lam 1998), or potassium (Chang and Lam 1998), uptake of 5-HT is significantly decreased, and when $\mathrm{Cl}^{-}$is replaced with acetate (Lingjaerde 1969), isothionate (Gu et al. 1994; Chang and Lam 1998; Oz et al. 2010b), and fluoride (Chang and Lam 1998), uptake is also decreased, highlighting the importance of the co-transport of these ions with 5-HT. 


\subsection{The Kappa Opioid Receptor}

\subsubsection{Introduction}

The KOPr is a PTX-sensitive (Avidorreiss et al. 1995), 7-transmembrane GPCR that exerts its effects in the brain and in the intestines. There are currently three known subtypes of KOPr: KOPr-1, KOPr-2, and KOPr-3, with KOPr-1 the only subtype currently cloned (Horan et al. 1993; Yasuda et al. 1993; Heyliger et al. 1999). Binding of KOPr agonists, such as the endogenous peptide dynorphin, lead to a conformational change and activation of $\mathrm{G}$ protein trimers as described in Figure 1.4. This leads to a cascade of events resulting in decreased neuron excitability. KOPr activation leads to

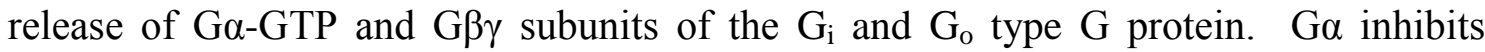
adenylyl cyclase, leading to decreased cAMP and decreased PKC activation (Avidorreiss et al. 1995). Continuous treatment of cells with a KOPr agonist leads to an immediate decrease in adenylyl cyclase activity, but when the agonist is removed, cAMP 'overshoot' occurs. This may contribute to tolerance to $\mathrm{KOPr}$ agonists (Avidorreiss et al. 1995). The released G $\beta \gamma$ binds directly to the G-protein-gated, inwardly rectifying potassium channel $\left(\mathrm{K}_{\mathrm{ir}} 3\right)$. Binding of $\mathrm{G} \beta \gamma$ activates the channel, leading to increased intracellular $\mathrm{K}^{+}$and cell hyperpolarisation (Clayton et al. 2009). Once the $\mathrm{G} \alpha$ subunit of the G protein is hydrolysed back to GDP, G $\beta \gamma$ dissociates from the channel and rebinds $\mathrm{G} \alpha$, deactivating the channel. It has been found that KOPr activation regulates $\mathrm{K}_{\mathrm{ir}} 3.1$ by phosphorylation of tyrosine 12 of the channel (Clayton et al. 2009). The $\mathrm{G} \beta \gamma$ subunit also causes decreased $\mathrm{Ca}^{2+}$ currents through direct binding to N, L and P/Q type $\mathrm{Ca}^{2+}$ channels (Rusin et al. 1997). This pathway is thought to cause the analgesic effects of KOPr agonists, as it leads to a decrease in $\mathrm{Ca}^{2+}$ dependent release of neurotransmitters. Activation of the KOPr by agonists leads to a multitude of effects, including analgesia, immunomodulation, hypothermia, dysphoria, anhedonia, and water diuresis (Liu-Chen 2004). Inhibition of the KOPr by antagonists causes antidepressant and anti-anxiety behaviours (Carlezon et al. 2009).

Dynorphin is the endogenous agonist for the KOPr. It is a highly basic peptide that is cleaved from prodynorphin by proprotein convertase 2 (Marinova et al. 2005). Prodynorphin can be cleaved into several types of active dynorphin: big dynorphin, dynorphin $\mathrm{A}, \mathrm{B}$, or $\alpha / \beta$-neo-endorphin. Dynorphin $\mathrm{A}$ is the most active form and preferentially activates KOPr, followed by MOPr and the delta opioid receptor (DOPr) (Merg et al. 2006). Dynorphin is found throughout the brain, in the hypothalamus, medulla, pons, spinal cord and midbrain of rats (Goldstein and Ghazarossian 1980) and 
is involved in many processes such as neuroendocrine regulation, pain regulation, motor activity, cardiovascular function, respiration, temperature regulation, feeding behaviour, and stress (Koob 2008). Activation of KOPr by dynorphin leads to depressive behaviours, anxiety, and increased drug seeking behaviours (Liu-Chen 2004).

\subsubsection{KOPr desensitisation}

Chronic KOPr agonism by the endogenous agonist dynorphin, or synthetic agonists such as $\mathrm{U} 50,488 \mathrm{H}$ or $\mathrm{U} 69,593$, leads to receptor desensitisation. The $\mathrm{G}$ protein is released from KOPr, and KOPr is phosphorylated by a GPCR kinase GRK3 (McLaughlin et al. 2003b), leading to the recruitment of non-visual arrestins. This causes a decrease between receptor and $\mathrm{G}$ protein coupling, leading to receptor desensitisation. KOPr is endocytosed by a clatherin and dynamin dependent pathway where it is then either dephosphorylated and recycled, or targeted to the lysosomal and proteasomal pathways for degradation (Chen et al. 2006). Human KOPr is phosphorylated on ser358; whereas, rat KOPr is phosphorylated on ser369. Rats and humans give different responses to KOPr agonists (Li et al. 2002). Rat and human KOPr expressed in Chinese hamster ovary $(\mathrm{CHO})$ cells are differently regulated by the KOPr agonist U50,488H, with phosphorylation and desensitisation of human KOPr but not rat $\mathrm{KOPr}$ (Li et al. 2002). This study also found that the N-terminal 7 residues of dynorphin A (1-17) were crucial for desensitisation. Another study using rat KOPr expressed in $\mathrm{CHO}$ cells found that peptide ligands of KOPr induced internalisation of rat KOPr, but nonpeptide ligands did not, even though both activated MAPK pathways (Jordan et al. 2000). There have been a number of studies demonstrating downregulation of rat KOPr with nonpeptide ligands (McLaughlin et al. 2003b; Bruchas et al. 2006; Clayton et al. 2009), and these differences are thought to be due to differences in the cell systems used.

\subsubsection{Synthetic KOPr agonists}

There are two main synthetic arylacetamide KOPr agonists: (trans- $( \pm)-3,4$-dichloro-Nmethyl-N-(2-[1-pyrrolidinyl]-cyclohexyl) benzeneacetamide methanesulfonate

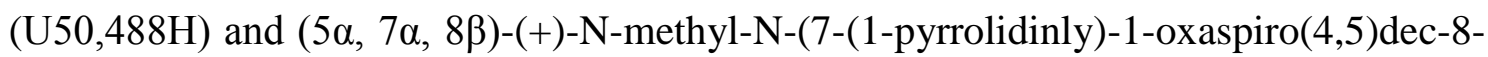
yl)benzeneacetamide (U69,593). U50,488H was first synthesized in the 1970's before KOPr had been discovered, and was recognised to be a specific KOPr agonist in the 1980's (Von Voigtlander and Lewis 1982). U69,593 was synthesised based on the structure of U50,488H (Figure 1.5) (La Regina et al. 1988). Both are potent KOPr 
agonists, with $\mathrm{U} 50,488 \mathrm{H}$ and $\mathrm{U} 69,593$ having $\mathrm{Ki}$ values of $0.70 \pm 0.09 \mathrm{nM}$ and 0.89 $\mathrm{nM}$, respectively when inhibiting $\left[{ }^{3} \mathrm{H}\right] \mathrm{U} 69,593$ binding in monkey brain membranes (Emmerson et al. 1994).

\subsubsection{Synthetic KOPr antagonists}

There are three common KOPr-1 specific antagonists: nor-binaltorphimine (nor-BNI) (Portoghese et al. 1987), 5'-guanidinonaltrindole (GNTI) (Jones and Portoghese 2000), and (3R)-7-hydroxy- $N-((1 S)-1-\{[(3 R, 4 R)-4-(3-h y d r o x y p h e n y l)-3,4-d i m e t h y l-1-$ piperidinyl]methyl\}-2-methylpropyl)-1,2,3,4-tetrahydro-3-isoquinolinecarboxamide (JDTic) (Thomas et al. 2001). nor-BNI, JDTic and GNTI are selective KOPr antagonists with Ki binding values to the KOPr of $1.09 \pm 0.14 \mathrm{nM}, 0.32 \pm 0.05 \mathrm{nM}$, and $0.18 \pm 0.1 \mathrm{nM}$, respectively with 60-, 10-, and 200-fold decreased binding to MOPr, and 80-, 1000-, and 380-fold decreased binding at DOPr (Jones and Portoghese 2000; Carroll et al. 2004). All three antagonists exhibit long-lasting effects on the KOPr, with nor-BNI exhibiting antagonistic effects for up to 28 days (Horan et al. 1992) and JDTic and GNTI for up to 14 days (Negus et al. 2002; Carroll et al. 2004).

Treatment of cultured cells with KOPr antagonists also causes up-regulation of transfected rat KOPr and human KOPr due to post-transcriptional modification (Chen et al. 2006; Wannemacher et al. 2007). The treatment of animals with antagonists such as nor-BNI has been shown to have anti-depressant and anti-stress effects (Mague et al. 2003; McLaughlin et al. 2003a; Knoll et al. 2007). Therefore, this pathway could be targeted to create anti-depressants rather than targeting the 5-HT pathway as with most other current therapies. A recent meta-analysis study carried out by Kirsch et al (2008) highlighted the importance of further research into the action of anti-depressants. The study concluded that the selective serotonin reuptake inhibitors (SSRI's) that are currently used as anti-depressants have a lesser effect than first thought (Kirsch et al. 2008). The additions of new anti-depressants that target different pathways are therefore important in increasing the recovery statistics for depression.

\subsubsection{KOPr activation pathways}

Activation of the KOPr by agonists leads to the activation of a number of MAPK cell signalling pathways. The known pathways are summarised below. 


\subsubsection{ERK1/2}

ERK $1 / 2$ is the most studied MAPK activated by KOPr. ERK1/2 is phosphorylated by MEK1/2 (Assenio et al. 2006), and its activation has been shown to be $\mathrm{P} 13 \mathrm{~K}, \mathrm{PKC} \zeta$ and

$\mathrm{Ca}^{2+}$ dependent (Fukuda et al. 1996; Belcheva et al. 2005). In mice, repeated forced swim stress led to activation of ERK1/2 which was GRK3-independent and was not seen in KOPr knockout mice, or when KOPr was inhibited by the KOPr antagonist norBNI (Bruchas et al. 2008). Forced swim stress is often used to induce depressive-like behaviour and can lead to relapse of drug seeking behaviours in laboratory animals. Their study demonstrated the involvement of KOPr and ERK1/2 in these behaviours. In rat primary astrocytes, activation of KOPr by the classic agonist U69,593 and the novel agonist MOM-SalB (DS-3-216) led to a G $\beta \gamma$ dependent biphasic activation of ERK1/2. The early phase was seen 5-15 min after agonist addition and was $\beta$-arrestin independent; whereas, the late phase was seen after $2 \mathrm{~h}$ and was $\beta$-arrestin dependent. As seen in other studies, this activation caused proliferation of these cells (Belcheva et al. 2005; McLennan et al. 2008). However, in immortalised rat astrocytes, U69593 still had these effects but MOMSalB (DS-3-216) only induced early phase activation of ERK1/2, suggesting that a different mechanism occurs in immortalised cells with this novel agonist. Activation of ERK1/2 by KOPr agonism has also been shown to encourage mouse embryonic stem cells to undergo limited proliferation and to differentiate into neural progenitor cells (Kim et al. 2006). Treatment of these neural progenitor cells with U69,593 led to a decrease in their proliferation. A follow up study investigated the effects of $\mathrm{KOPr}$ agonism on neural progenitor cell differentiation and found that KOPr inhibits neurogenesis and astrogenesis, but stimulates olidgodendrogenesis, and that this is regulated by both ERK1/2 and p38 MAPK (Hahn et al. 2010).

\subsubsection{2 p38 MAPK}

p38 MAPK is involved in stress and has been shown to control aversion in rat models (Bruchas et al. 2007a). p38 MAPK activation in transfected cell models, primary mouse brain cultures, and mice is GRK3 and arrestin dependent (Bruchas et al. 2006; Clayton et al. 2009). It appears that phosphorylation of KOPr by GRK3 after agonism, and then the recruitment of arrestin is necessary before phosphorylation of p38 MAPK occurs (Bruchas et al. 2006). In mice that have undergone repeated swim stress, there were increases in activation of both KOPr and p38 MAPK, and the activation of p38 MAPK was not observed in KOPr knockout mice. Furthermore the conditioned place 
aversion caused by U50,488H was blocked when p38 MAPK was inhibited (Bruchas et al. 2007a), suggesting a direct role of P38 MAPK in this aversion. In mice that had partial sciatic nerve ligation, which serves as a model for physiological stress, there was an increase in the potassium channel $\mathrm{K}_{\mathrm{ir}} 3.1$ phosphorylation that was not seen in KOPr or GRK3 knockout mice. In a transfected cell system an increase in $\mathrm{K}_{\mathrm{ir}} 3.1$ phosphorylation was also observed after U50,488H treatment, and this was p38 MAPK and src kinase dependent. This suggests that KOPr agonism leads to p38 MAPK activation which then activates src kinases, which phosphorylate $K_{\mathrm{ir}} 3.1$ leading to receptor desensitisation (Clayton et al. 2009). KOPr activation of p38 MAPK in the dorsal raphe nuclei also causes conditioned place aversion in mice (Land et al. 2009), further strengthening the role of p38 MAPK as a modulator of stress and aversion.

\subsubsection{3 c-Jun N-terminal kinase}

The c-Jun N-terminal kinase (JNK) pathway has been implicated in stress, pain, apoptosis, and inflammatory responses (Leppa and Bohmann 1999). There has been little research conducted on JNK and KOPr, but what has been conducted shows that KOPr activation leads to JNK activation. The KOPr antagonists nor-BNI and JDTic both have a very long in vivo action, and in HEK-293 cells stimulate JNK phosphorylation, interestingly through a PTX insensitive manner (Bruchas et al. 2007b). nor-BNI also activates JNK in the mouse striatum and spinal cord, and this effect is not seen in KOPr knockout mice (Bruchas et al. 2007b). In COS7 cells co-transfected with JNK and KOPr, activation of KOPr with $\mathrm{U} 50,488 \mathrm{H}$ leads to an increase in JNK phosphorylation that is independent of P13K (Kam et al. 2004b). A further study proposed that KOPr activation of JNK occurred through a $\mathrm{G}_{\mathrm{i} / \mathrm{o}} \mathrm{G}_{\beta \gamma}$, src tyrosine kinase, focal adhesion kinase and Rac, Cdc42 and son-of-sevenless (sos) dependent pathway (Kam et al. 2004a). KOPr antagonists such as nor-BNI are thought to act by disrupting the KOPr signaling pathway, possibly by activation of the JNK phosphorylation pathway (Bruchas et al. 2007b).

\subsubsection{KOPr and dopamine}

It has been known for over 20 years that KOPr has an inhibitory role on dopaminergic neurons in the rat brain (Di Chiara and Imperato 1988b; Mananares et al. 1991). These initial studies found that KOPr agonists cause a decreased release of DA in the NAc and dorsal caudate in freely moving rats and decreased motor activity (Di Chiara and Imperato 1988b; Kunihara et al. 1993), and that KOPr agonism inhibits stimulated 
dopaminergic neurons in the nigrostriatal, mesolimic, tuberoinfundibular, and tuberohypophysial systems (Mananares et al. 1991). These effects were reversible by KOPr antagonists, demonstrating that they were KOPr mediated. The effects of KOPr on DA are thought to be in part due to an interaction with DRD1 and DRD2 receptors. The KOPr agonist spiradoline was substituted by rats with the DRD2 receptor angatonist sulpiride in drug discrimination tests, suggesting that KOPr effects are modulated through inhibition of DRD2 receptors (Ohno et al. 1992). The inhibition of DA release by presynaptic KOPr has also been shown in rabbit caudate nucleus slices. This effect was shown to be PTX sensitive. While DRD2 receptors were also found to decrease DA release, there was no direct link found for a specific interaction between the action of KOPr and DRD2 receptors (Jackisch et al. 1994). KOPr also regulates DRD1 receptors in the ventral striatum in rats depleted of DA (Steiner and Gerfen 1996). Studies carried out using fast-scan voltammetry have also shown U50,488H inhibition of DA overflow in rat neostriatal slices. After several doses of U50,488H, this effect was decreased, suggesting receptor desensitisation (Schlosser et al. 1995).

Repeated exposure to $\mathrm{U} 69,593$ has the opposite effects to acute exposure. In vivo microdialysis studies in the rat NAc (Fuentealba et al. 2006) and medial PFC (Fuentealba et al. 2010) found that treatment with U69,593 once a day for four days led to no change in basal DA levels, but an increase in $\mathrm{K}^{+}$-stimulated DA levels compared to control rats. The DRD2 receptor agonist quinpirole decreased both basal and $\mathrm{K}^{+}$evoked DA levels in control rats but had no effect in U69,593-treated rats (Fuentealba et al. 2006). This suggests that repeated exposure to KOPr agonists leads to changes in the dopaminergic neuron response to stimulation in the NAc.

Other studies investigating the link between DRD2/3 receptors and KOPr have found that chronic administration of U69,593 along with a postsynaptic dose of quinpirole leads to increase in sensitisation to quinpirole (Perreault et al. 2006). When presynaptic doses of quinpirole were given alongside U69,593 a switch from decreased locomotor to increased locomotor responses were observed. A follow up study looked at the effect of these treatments on gene expression of DRD1, DRD2, DAT and dynorphin after 10 injections of U69,593 followed by quinpirole (Perreault et al. 2006). After 10 injections there was decreased DRD2 mRNA in the substantia nigra and increased dynorphin mRNA in the striatum and NAc core (Perreault et al. 2007). These authors concluded that repeated treatment caused an increase in locomotor activity as seen in the previous 
study due to the activation of DRD1 and dynorphin neurons and the inhibition of presynaptic DRD2 function.

\subsubsection{KOPr and serotonin}

There is also evidence to suggest that KOPr also regulates 5-HT in the brain. Treatment with KOPr agonists causes depression in animal models which is inhibited by the use of the SSRI fluoxetine (A. Morani, personal communication). Another study demonstrated that the KOPr agonists salvinorin A and U69,593 lead to inhibition of $\mathrm{K}^{+}$-evoked 5-HT release in mouse hippocampal synaptosomes (Grilli et al. 2009). U69,593 also has been shown to inhibited RTI-55 induced cocaine seeking in rats but not WIN35,428 induced cocaine seeking (Schenk et al. 2000). This implies that the effect of U69,593 on cocaine seeking in rats is in part due to interactions with SERT. This was investigated further with cocaine self-adminstering rats pretreated with fluoxetine, or the KOPr aognists spiradoline or enadoline. Pretreatment led to inhibition of cocaine selfadministration, and this effect was reversed by the 5-HT(1A) agonist R(+)8-hydroxy-2(di-n-propylamino)tetralin (Ruedi-Bettschen et al. 2010). These studies demonstrate that not only can KOPr regulate DA in the synapse, but also 5-HT.

\subsubsection{KOPr and drug addiction}

Many studies have shown that after drug use the KOPr/dynorphin system is upregulated, leading to drug seeking and craving during withdrawal (Mash and Staley 1999; Shippenberg et al. 2007). Rat studies have shown that a single dose of amphetamine $(2.5 \mathrm{mg} / \mathrm{kg})$ leads to a release of dynorphin and long-lasting desensitisation of KOPr in the NAc shell (Turchan et al. 1998; Xia et al. 2008). Amphetamine $(2.5 \mathrm{mg} / \mathrm{kg})$ also caused an increase in mRNA expression of preprodynorphin in the striatum that was inhibited by a single dose of the KOPr agonist U69,593. This treatment also led to a decrease in the amphetamine increased locomotor response of the rat (Tzaferis and McGinty 2001). In post-mortem studies of human cocaine users there are large increases in dynorphin in the caudate, putamen, and ventral pallidum (Hurd and Herkenham 1993; Frankel et al. 2008) and increases in KOPr in the caudate, putamen, and NAc (Hurd and Herkenham 1993; Mash and Staley 1999). This effect was also seen in rats that had been treated chronically with cocaine, with increases in KOPr seen in the rostral cingulate cortex, rostral caudate putamen, and the VTA (Unterwald et al. 1994). 
KOPr agonists and antagonists have demonstrated their ability to alter the effects of drugs of abuse and withdrawal in animal studies. The KOPr agonist U69,593 inhibited the cocaine-stimulated increase in NAc basal DA overflow and cocaine behaviour when administered alongside cocaine for 3 days (Heidbreder and Shippenberg 1994). A single dose of U50,488H before a single dose of cocaine inhibited the increase of DA overflow in the NAc by $50 \%$ in awake and freely moving rats, and this effect was norBNI reversible (Maisonneuve et al. 1994). In morphine or cocaine self-administering rats a single injection of the KOPr agonists $\mathrm{U} 50,488 \mathrm{H}$ or spiradoline led to extinction of drug taking after 1 day, which is still seen in some rats up to 6 days later, and the effect is norBNI reversible (Glick et al. 1995). Neither agonist had an effect on lever pushing for water, suggesting the effect is specific for drugs of abuse.

Classic KOPr agonists such as $\mathrm{U} 69,593$ and $\mathrm{U} 50,488 \mathrm{H}$ have been shown in vivo to attenuate self-administration of cocaine, amphetamine and alcohol in rodents (Heidbreder et al. 1993; Heidbreder et al. 1998; Schenk et al. 1999; Collins et al. 2001a; Schenk and Partridge 2001; Mague et al. 2003; Knoll et al. 2007; Zhang et al. 2007; Tomasiewicz et al. 2008; Logrip et al. 2009; Sun et al. 2010). Classical KOPr antagonists in vivo have anti-depressant/anti-stress effects that prevent rodents from relapsing after attenuation of self-administration of cocaine (Redila and Chavkin 2008). Unfortunately, these classical agonists and antagonists lead to side effects such as diuresis, depression, and dysphoria and therefore have not successfully progressed to clinical trials (Thompson et al. 2000). Thus, novel compounds that activate the KOPr pathway have the potential of attenuating addiction through modulation of the DAT, leading to cessation of drug taking (agonists) and prevention of stress-related relapse (antagonists).

Cellular and behavioural studies have shown that the action of KOPr agonists attenuates the addictive properties of cocaine. For example, Thompson et al. (2000) showed that the acute treatment of rats with U69,593 and cocaine caused an increase in DA uptake by DAT in the NAc, decreasing the rewarding effects of cocaine. They also observed that U69,593 opposed the effects of cocaine withdrawal in the NAc after repeated administration of U69,593 with cocaine (Thompson et al. 2000). Collins et al. (2001) carried out acute and chronic experiments to observe the locomotor activity of rats treated with cocaine and U69,593. They found that prior treatment of rats with U69,593 decreased locomotion following cocaine exposure compared to animals pre-treated with 
vehicle (Collins et al. 2001a; Collins et al. 2001b). In C57BL/6J mice, dynorphin A (117) inhibited the acute increase in DA in the striatum after cocaine treatment, and also inhibited cocaine conditioned place preference and locomotor activity (Zhang et al. 2004). In vivo microdialysis studies of abstinent rats that had been treated with cocaine for five days and intracranial administration of U69,593 for days 3-5 showed that U69,593 treatment prevented the basal increase in DA levels in the NAc but not PFC after 3 days of abstinence (Chefer et al. 1999). Intracranial administration of U69,593 into the NAc also prevented cocaine induced locomotor sensitisation. This study suggested that NAc KOPr plays a role in the DA and locomotor effects of cocaine. In vivo microdialysis studies in the medial PFC of rats that had been given cocaine for 10 days and then abstained for 3 days found that U69,593 inhibited the sensitisation to cocaine when rats were given a single injection (Chefer et al. 2000). This treatment also inhibited the decreased DA levels evoked by cocaine and increased basal DA levels in the medial PFC. In medial PFC synaptosomes, U69,593 also inhibited the cocaineinduced increase in DA uptake. These data suggest that KOPr agonism has the ability to inhibit the early phase of abstinence from cocaine (Chefer et al. 2000). In cocaine self-administering rats, an acute injection of U50,488H into the VTA dose dependently decreased cocaine-induced reinstatement in a nor-BNI reversible manner, demonstrating that the VTA KOPr are involved in this inhibition (Sun et al. 2010). Mello and Negus (1998) have also shown that treatment of Rhesus monkeys with several different KOPr agonists, including U50,488, leads to decreased cocaine self-administration (Mello and Negus 1998). This demonstrates the therapeutic potential of KOPr agonists in breaking the addiction cycle.

The KOPr system has also been shown to modulate the locomotor effects of other drugs of abuse such as amphetamine (Gray et al. 1999; Vanderschuren et al. 2000) and nicotine (Hahn et al. 2000). Acute KOPr agonism inhibits the locomotor effects of amphetamine in rats (Gray et al. 1999; Vanderschuren et al. 2000), while chronic KOPr agonism leads to potentiation of amphetamines effects (Fuentealba et al. 2007). A four day treatment with the KOPr agonist U69,593 (once daily for four days, subcutaneous. $0.16 \mathrm{mg} / \mathrm{kg}$ days 1 and 2, $0.32 \mathrm{mg} / \mathrm{kg}$ day 3 and 4) caused increased locomotor response to amphetamine, as well as increasing amphetamine induced DA in the NAc (Fuentealba et al. 2007). However, when KOPr agonists were given alongside amphetamine for 5 days and then the locomotor effect measured after 3 weeks, the KOPr agonists had no effect on locomotor sensitisation, suggesting that KOPr is not 
involved in sensitisation in this model (Vanderschuren et al. 2000). The KOPr agonist U69,593 also inhibits the effect of neurotoxic doses of methamphetamine, with complete inhibition of the methamphetamine-induced decrease in basal and $\mathrm{K}^{+}$-evoked DA release in mice (El Daly et al. 2000). In wild-type mice treated with nor-BNI or in KOPr knockout mice, the effect of an acute ethanol exposure leads to augmentation of ethanol-induced increases in DA in the NAc, again demonstrating the modulation of DA by $\operatorname{KOPr}$ (Zapata and Shippenberg 2006).

In animal studies, relapse to cocaine seeking was induced by foot shock in mice, and this effect was reversed by KOPr antagonists, suggesting that the stress-induced relapse was modulated by the KOPr system (Beardsley et al. 2005; Redila and Chavkin 2008). The repeated forced swim test is a test used to measure depressive-like behaviour in mice, such as immobility and analgesia. This behaviour has been found to be KOPr dependent as it is blocked by KOPr antagonism and does not occur in KOPr or dynoprhin knockout mice (McLaughlin et al. 2003a; McLaughlin et al. 2006a). Mice that are exposed to the repeated forced swim test have increased cocaine conditioned place preference, and this effect is mimicked by U50,488H (McLaughlin et al. 2006a), indicating the effect of KOPr activation and stress on drug seeking in this model. Another stress test carried out in mice is the social defeat stress test, which induces analgesia, immobility, and a defeated postural response. This test also causes potentiation of cocaine conditioned place preference. Treatment of mice with nor-BNI inhibits these behaviours and blocks the stress-induced cocaine condition place preference (McLaughlin et al. 2006b). These studies all show that the KOPr is involved in addiction, with a particular role in stress-induced drug seeking and craving.

In the brain, KOPr plays a part in regulating the activity of DAT (Ramamoorthy et al. 2002a; Margolis et al. 2003; Margolis et al. 2006; Gehrke et al. 2008) and SERT (Schenk et al. 2000; Collins et al. 2002). Acute KOPr agonism leads to an increase in DAT function and a decrease in SERT function, and antagonism decreases DAT function and increases SERT function. While the exact mechanism of how this occurs is unknown, it has been shown that KOPr agonists not only inhibit DA release by interacting with DRD2 receptors (Acri et al. 2001) but also alter the activity of DAT and SERT in single cells (Kivell et al. 2004). These effects are caused by both activation of signalling pathways and also a physical interaction between KOPr and DAT (T. Shippenberg, unpublished data). 
While there are promising indications of KOPr effects in animal models of addiction, several KOPr agonists have been trialled in cocaine abusing humans and overall have shown no effect on drug taking behaviour. The KOPr agonist, enadoline, is tolerated well up to $80 \mu \mathrm{g} / 70 \mathrm{~kg}$ body weight, alone and in combination with cocaine (Walsh et al. 2001a; Walsh et al. 2001b), but enadoline did not alter drug seeking or taking behaviour (Walsh et al. 2001a). Another agonist, cyclazocine, had a possible effect on the acute effects of cocaine in patients with a history of cocaine and opiate use. After six treatments with the agonist over 3 weeks, the effects of a single dose of cocaine appeared to be decreased in the presence of cyclazocine compared to controls, although this was only seen as suggestive by the authors (Preston et al. 2004). Overall, this suggests that while these compounds have positive results in animals, in humans they do not have the same effect. However, as KOPr agonists cause decreased mood, they do have potential as anti-mania pharmacotherapy. For example, the partial KOPr agonist pentazocine decreased manic episodes in patients with bipolar disorder when two doses of $50 \mathrm{mg}$ were administered two hours apart (Cohen and Murphy 2008).

Though acute treatment with KOPr agonists leads to a non-addictive behaviour, chronic treatments have been shown to lead to tolerance, and once treatment has stopped addictive properties are often seen. This is thought to occur in three main stages: desensitization due to the phosphorylation of the receptors and uncoupling from $G$ proteins, internalization of the receptors, and finally down-regulation of the number of receptors present on the cell surface (Liu-Chen 2004). This occurs as the long-term inhibition of adenylate cyclase by the activated KOPr leads to the compensatory upregulation of cAMP through a different pathway. Once KOPr agonist treatment has stopped, this compensatory pathway, along with the natural activation of adenylate cyclase, leads to an increase in cAMP levels, and this leads to symptoms of dependence and withdrawal (Nestler and Aghajanian 1997).

The 'anti-addictive' effects that KOPr demonstrate are most likely also mediated through the serotonergic system as well as the dopaminergic system. A reinstatement study carried out in cocaine self-administering rats investigated if U69,593 could inhibit specific DAT inhibitors' induction of reinstatement. Interestingly, U69,593 did not block the effects of the DAT inhitibor GBR12909 on reinstatement in rats, but it did prevent reinstatement by both cocaine and the cocaine analogue RTI-55, suggesting that 
other pathways are involved with KOPr 'anti-addictive' effects. The authors suggested that the serotonergic system may be involved (Schenk et al. 2000). Another study looked at the effect of continuous infusion in rat brains of cocaine or specific DAT inhibitors on KOPr expression. Results showed that 14 days of continuous cocaine leads to an increase in KOPr expression in the NAc and caudate putamen, but continuous exposure to the specific DAT inhibitors GRB12909 and RTI-117 has no effect on KOPr expression (Collins et al. 2002). This suggests that the increase in KOPr expression is not due to DAT. Other studies have shown that depletion of 5-HT in the hippocampus led to the inhibition of the effects of U69,593 and cocaine on predynorphin mRNA expression (D'Addario et al. 2007), and that depletion of 5-HT in rats inhibits the effect of cocaine on locomotor activity in rats given repeated exposures to U69,593 (Zakharova et al. 2008b). These studies demonstrate that the serotonergic system is involved in the pathway between cocaine and KOPr. Reinstatement experiments in self-administering squirrel monkeys also show that both KOPr agonists and SERT inhibitors prevent cocaine priming reinstatement of drug seeking, further establishing a role for 5-HT in this pathway (Ruedi-Bettschen et al. 2010).

To conclude this section, KOPr agonists show anti-addictive properties in animal models of addiction and appear to exert this effect partially through the DA and 5-HT pathways in the brain. However, the classic KOPr agonists which have been trialled in human studies, have little or no effect on cocaine taking. Therefore, there is a need for the effects on drug addiction of novel KOPr agonists to be investigated, with further investigation on how they modulate the DA and 5-HT systems. 


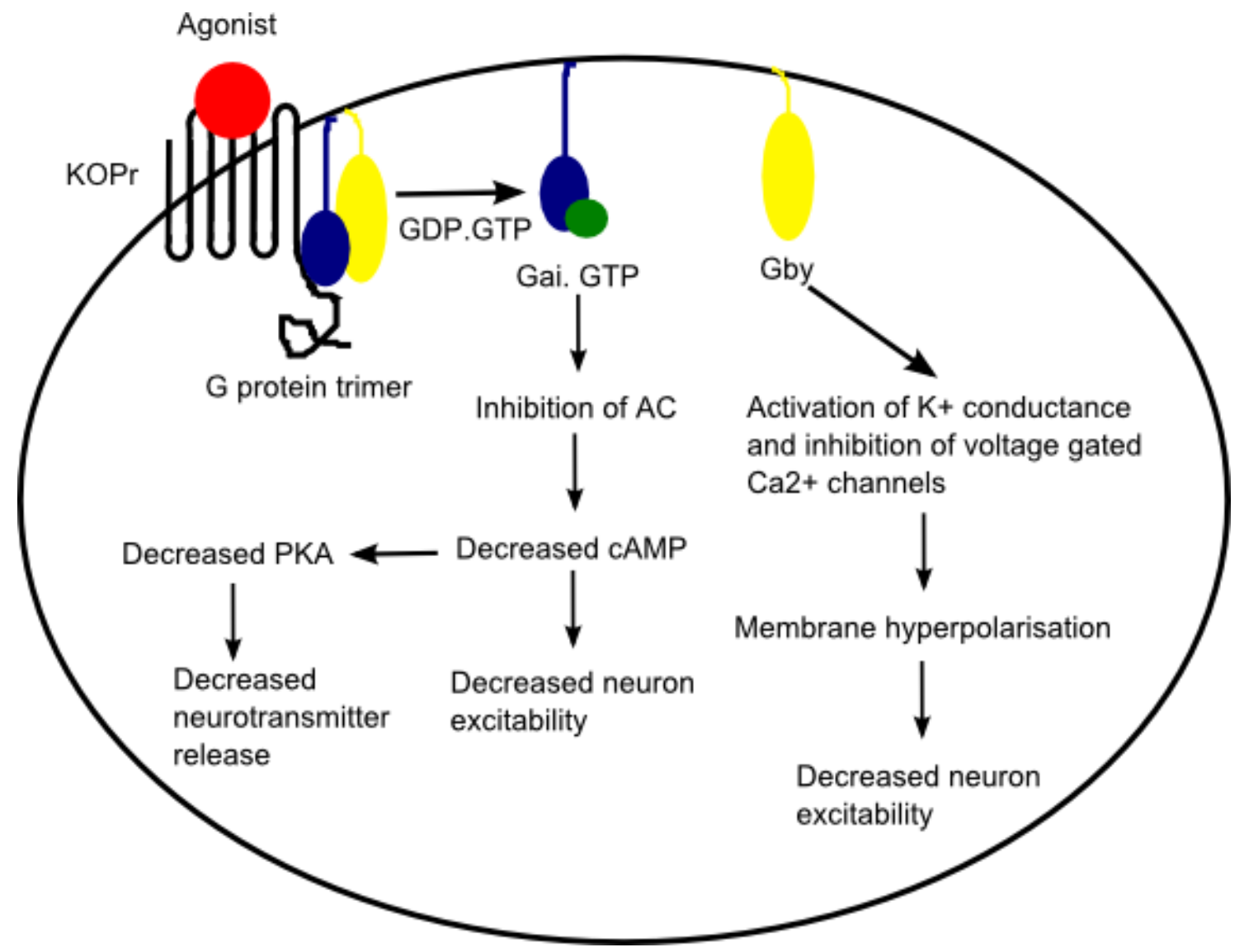

Figure 1.4: Activity of KOPr. The binding of a ligand to KOPr leads to a conformational change in the receptor. This allows the pertussis toxin-sensitive Gi protein trimer to replace

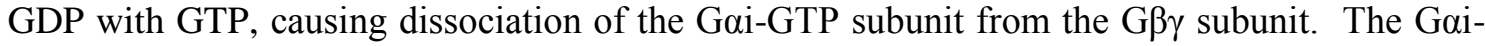
GTP subunit inhibits adenylate cyclase (AC), leading to a decrease in cAMP. This causes a decrease in neuron excitability and also a decrease in PKA which reduces neurotransmitter

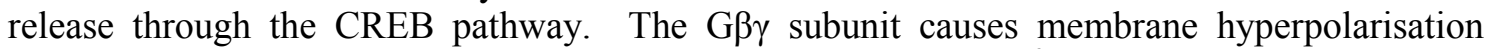
through the activation of $\mathrm{K}^{+}$conductance and inhibition of $\mathrm{Ca}^{2+}$ channels, thus decreasing neuron excitability. 
A<smiles>CN(C(=O)Cc1ccccc1)[C@@H]1CCCC[C@H]1N1CCCC1</smiles><smiles>[B]C1CCN([C@@H]2C[C@]3(CCCO3)CC[C@@H]2N(C)C(=O)Cc2ccccc2)C1</smiles>
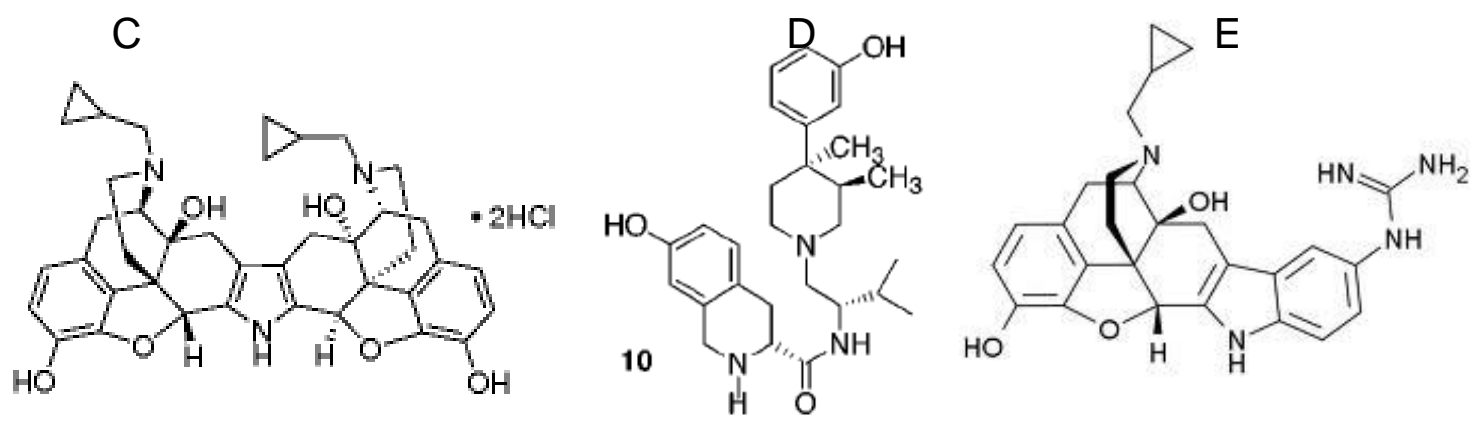

Figure 1.5: Structures of the classic KOPr agonists U50,488H (A) and U69593 (B) and antagonists nor-BNI (C), JDTic (D), and GNTI (E).
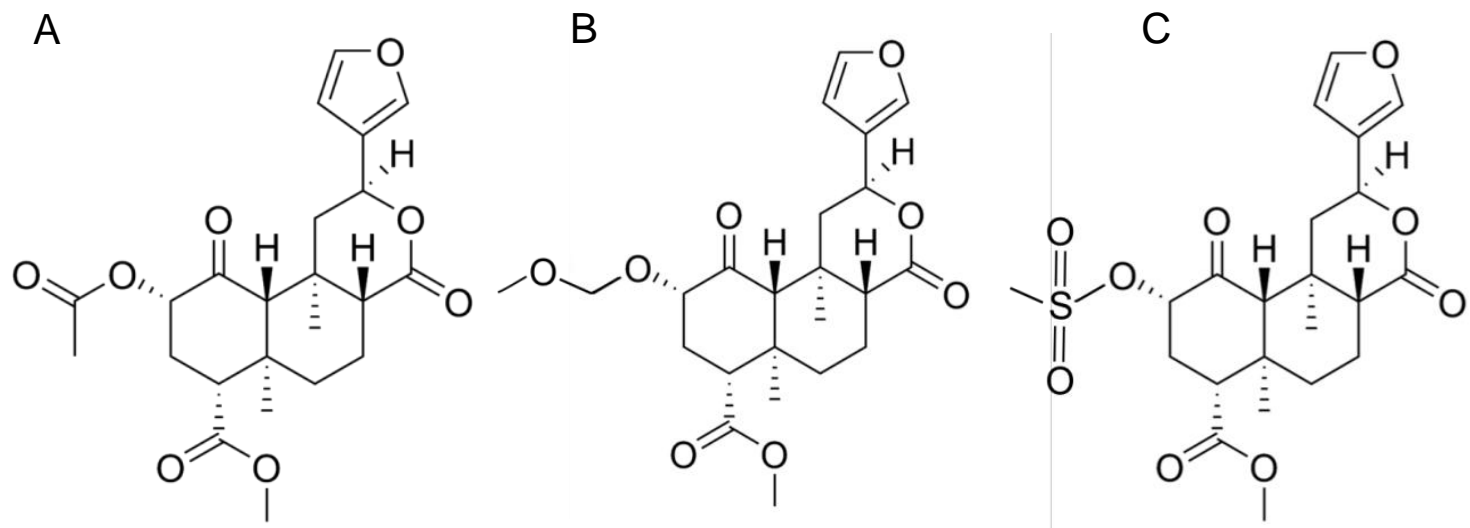

Figure 1.6: Structures of salvinorin A (A) and its analogues DS-1-240 (B) and DS3-216 (C). 


\subsection{Salvinorin A}

Salvinorin A (Figure 1.5) is a unique non-nitrogenous hallucinogen that is purified from the plant Salvia divnorum. Salvia divnorum is a rare member of the mint family and is traditionally used by Mazatec Indians in shamanistic rituals to induce short, intense periods of hallucination. Traditionally this hallucinogen is taken either by chewing fresh leaves, or by drinking the juice of crushed leaves, and it enters the body through the oral mucosa (Siebert 1994). When it is taken in this way it has a long and fairly mild duration of action (Valdes 1994). Salvinorin A has also recently become a recreational drug that is usually smoked in the Western world. It currently shows no addictive properties in people, even though in conditioned place preference studies it can induce addictive behaviour in zebra fish and rats (Braida et al. 2007; Braida et al. 2008). Salvinorin A is the most potent naturally occurring hallucinogen, having a similar potency as the synthetic drugs lysergic acid diethylamide (LSD) and 4-bromo2,5-dimethoxy-phenylisopropylamine (DOB) (Siebert 1994; Valdes 1994). Salvinorin A induces a short-lasting, intense period of hallucination when smoked that persists for a few minutes to an hour. It is rapidly hydrolysed by carboxylesterase to the weak KOPr agonist salvinorin B (Schmidt et al. 2005a; Teksin et al. 2009; Tsujikawa et al. 2009). Salvinorin A is not substituted for its discriminative stimuli by LSD or ketamine in rats demonstrating its unique mode of action (Killinger et al. 2010). Salvinorin A is a controlled substance in Australia, Belgium, Canada, Denmark, Estonia, Finland, Italy, Japan, Russia, Spain, and Sweden, with many of the states of the USA also banning salvinorin A.

There have been several surveys carried out questioning salvia users on their subjective thoughts on salvia use. Surveys in the USA show that salvia is a more recently accepted recreational drug, with smoking the most common way of taking it, and the effects being a fast onset and a short duration (Gonzalez et al. 2006). An interview of American college students found that $4.4 \%$ of students surveyed had used salvia, and that white male fraternity members who had a history of heavy episodic periods of drinking were most likely to take it (Lange et al. 2008). Another study reported that the effects of salvia were more like marijuana than classic hallucinogens such as LSD (Albertson and Grubbs 2009). The majority of salvia users reported no salvia-induced psychosis, and said that if saliva was to become illegal in their state they would still continue to use it (Sumnall et al. 2010). There has been one person who was reported to use salvia successfully as an anti-depressant (Hanes 2001), and one report of persistant 
psychosis and paranoia after smoking salvia (Przekop et al. 2009). Overall, these surveys demonstrate that salvia is becoming increasingly popular, and that the majority of users find it to be a safe drug to take, with only one reported incident of serious side effects.

Salvinorin A is biologically unique hallucinogen, as it has been shown to be a potent and selective KOPr agonist (Roth et al. 2002; Sheffler and Roth 2003; Chavkin et al. 2004) rather than targeting $5-\mathrm{HT}_{2 \mathrm{~A}}$ serotonergic receptors as is the case for classical hallucinogens. It is also unique as a KOPr agonist as it has a non-nitrogenous structure (Figure 1.5). Studies have shown that salvinorin A is a selective KOPr agonist and shows no affinity for MOPr or DOPr (Roth et al. 2002). Therefore, salvinorin A has the potential to be chemically modified to form semi-synthetic analogues that act as KOPr agonists and antagonists, introducing a new class of compounds that have potential as therapeutic drugs to treat addiction and relapse.

A number of experiments documenting salvinorin A's action in the cell and in vivo have been published. A cellular study carried out by Wang et al. (2005) characterized salvinorin A's pharmacological activity alongside other KOPr agonists (Wang et al. 2005). They showed that salvinorin A had a Ki of $7.9 \mathrm{nM}$. Roth et al. (2002) showed that salvinorin $\mathrm{A}$ had a $\mathrm{Ki}$ value of $16 \mathrm{nM}$, and that it was a full agonist of KOPr. Several studies have also reported that the discriminative stimulus effects of salvinorin $\mathrm{A}$ are mediated by KOPr in both non-human primates and rodents (Butelman et al. 2004; Willmore-Fordham et al. 2007; Baker et al. 2009; Butelman et al. 2010) and that MOPr agonists, DOPr agonists, and other hallucinogens such as psilocybin or ketamine are not generalised (Butelman et al. 2010). This demonstrates that the effects of salvinorin A are mediated through the KOPr pathway, rather than MOPr, DOPr, or the serotonergic pathways. Salvinorin A has overlapping effects with the non-selective NMDA receptor antagonist ketamine, as JDTic, a KOPr-1 antagonist, inhibited some of ketamine's effects in a rat model (Nemeth et al. 2010). Carlezon et al (2006) carried out behavioural experiments with rats given salvinorin A and showed that administration of salvinorin A before a number of behavioural tests led to behaviour that was consistent with effects characteristic of other depressant drugs (Carlezon et al. 2006). The same results were gained in studies with salvinorin A in mice (Zhang et al. 2005). These experiments showed how salvinorin A exerted its effects through the KOPr and that its physiological effects were consistent with other KOPr agonists. 
Studies in rodents have shown that salvinorin A decreases DA overflow and locomotor activity caused by cocaine administration (Zhang et al. 2005; Chartoff et al. 2008; Gehrke et al. 2008). Salvinorin A also attenuates self-administration in rats when acutely administered before cocaine reinstatement tests (Morani et al. 2009). Recent studies in rats have shown that an active dose of salvinorin $\mathrm{A}(0.32 \mathrm{mg} / \mathrm{kg})$ leads to a decrease in phasic DA release, with no changes in uptake in the NAc. This occurred alongside a decrease in motivation of these animals, measured by intracranial selfstimulation (Ebner et al. 2010). Another study found that salvinorin A stimulates DRD2 receptors, which may account for the results seen in the previous study (Seeman et al. 2009). In mice, a single dose of salvinorin A leads to a rapid, short-lived induction of sedation or motor incoordination which is nor-BNI reversible (Fantegrossi et al. 2005). This effect was similar to that induced by U69,593. KOPr agonists cause increased pain thresholds (antinociception). Mice exposed to a single dose of salvinorin A demonstrate a nor-BNI reversible antinociception to both thermal and chemo-nociceptive stimuli, with the peak seen after $10 \mathrm{~min}$ of salvinorin A administration (John et al. 2006; McCurdy et al. 2006). Antinociception and hypothermia after salvinorin A treatment is not seen in KOPr-1 knockout mice, suggesting that salvinorin A binds specifically to KOPr-1 receptors (Ansonoff et al. 2006). Another study that links the effects of salvinorin A to DA was conducted in mouse hippocampal synaptosomes and showed that salvinorin A presynaptically mediates norepinephrine, 5-HT, and DA exocytosis (Grilli et al. 2009).

Salvinorin A has a very short half-life as it is quickly metabolised into the more stable but inactive salvinorin B derivative, and this may be problematic for development of salvinorin A into an anti-addiction therapeutic. A PET study carried out in baboons showed that C-11 labelled salvinorin A peaked in the cerebellum and cortex within $40 \mathrm{~s}$ of administration (i.v.) and had a half-life of only $8 \mathrm{~min}$ (Hooker et al. 2008). These data mirror that seen with human users, with salvinorin A showing a fast onset and short duration. The same group recently used PET and a brain metabolic tracer to find where in the brain salvinorin A (i.p.) exerts its effects (Hooker et al. 2009). They found that salvinorin A had an effect on brain metabolism in areas that contain high KOPr, for example the periaqueductal grey, the vermis of the cerebellum, the caudate putamen, and the bed nucleus of the striata terminalis. Interestingly, brain metabolism is also increased in areas that are not densely KOPr populated, for example, the hippocampus 
and medial brainstem. The authors hypothesized that these other regions may be involved in the physiological effects of salvinorin A and may be areas that can be further studied with new analogues. After an intravenous injection of salvinorin A into Rhesus monkeys it took only $1 \mathrm{~min}$ for salvinorin A to reach the central nervous system, and 1 to 2 min to cause facial relaxation and ptosis (drooping of the eyelid) (Butelman et al. 2009). Physiological and histological studies on the effect of salvinorin A on rats and mice have shown that salvinorin A exhibits low toxicity, even at doses much higher than those that humans administer (Mowry et al. 2003).

Recent studies have interestingly shown that low doses of salvinorin A have a rewarding effect in rats and zebrafish, but higher doses are aversive (Braida et al. 2007; Braida et al. 2008; Braida et al. 2009). This rewarding effect was attenuated by pretreatment with the cannabinoid receptor 1 (CB1) antagonist, rimonabant. This suggests that at low doses, the KOPr interacts with the $\mathrm{CB} 1$ receptor to cause these rewarding effects. Another later study, however, suggests that these affects are due to rimonabant acting at KOPr to inhibit its effects (Walentiny et al. 2010). Locomotor studies using salvinorin A and a DRD1 receptor agonist SKF 82958 showed that acute administration of salvinorin A blocked the locomotor stimulation effects of the agonist in the dorsal striatum but that after chronic salvinorin A treatment the locomotor stimulation was increased. These results parallel those found with cocaine locomotor studies, and show that salvinorin A regulates the locomotor effects of cocaine through DRD1 receptor signalling (Chartoff et al. 2008). Locomotor studies in rats with salvinorin A coadministered with the DRD2/DRD3 receptor agonist quinpirole showed that salvinorin A produced a bidirectional effect, with low doses causing attenuation of the locomotor effect, middle doses causing no effect, and high doses causing potentiated effects (Beerepoot et al. 2008). This biphasic response has not been seen with other KOPr agonists, possibly because of the narrow range of concentrations used, and further investigation is needed. It has also been suggested by Rothman et al. that salvinorin A allosterically inhibits binding of ligands to MOPr (Rothman et al. 2007). In a series of experiments, it was shown that salvinorin A weakly inhibits the binding of DAMGO and diprenorphine to MOPr, suggesting that salvinorin $\mathrm{A}$ is not as selective as first thought. These experiments demonstrate the increasing interest in salvinorin A and how it exerts its effects within the brain. 
Salvinorin A is also unique in that it does not cause water diuresis in rats, possibly due to its short duration of action (Inan et al. 2009). Salvinorin A is traditionally used as a medication for treatment of gastrointestinal problems such as diarrhoea (Valdes et al. 1983). Studies carried out in guinea pigs and mice have shown that salvinorin A and Salvia divinorum leaf extract decrease enteric cholinergic transmission, which confirms its use as an anti-diarrhoeic (Capasso et al. 2006). In mouse inflamed intestines, salvinorin A leads to a decreased motility of a fluorescent marker, and this decrease in motility was inhibited by both nor-BNI and rimonabant, the $\mathrm{CB} 1$ receptor inhibitor (Capasso et al. 2008b). This CB1 and KOPr cross-talk was only observed in inflamed intestines and not in normal mouse intestines (Capasso et al. 2008a). Another study investigated the effect of salvinorin A on colonic transit in mice and showed that in vivo salvinorin A prolonged colonic propulsions and slowed upper gastrointestinal transit, and in vitro it acted to inhibit contractions of the isolated colon, stomach, and ileum. These effects were mediated by KOPr and CB1 and CB2 in the in vitro studies and KOPr in the in vivo studies, with a slight activation at CB2 (Fichna et al. 2009).

There have been a number of studies carried out with the aim of detecting salvinorin A and its metabolites in blood, plasma, and urine samples so that the pharmacokinetics of salvinorin A can be investigated. One method employed was liquid chromatographymass spectrometry/atmospheric pressure chemical ionisation (Schmidt et al. 2005b) which could accurately detect salvinorin $A$ at concentrations of $2 \mathrm{ng} / \mathrm{mL}$ to $100 \mathrm{ng} / \mathrm{mL}$ in human and rhesus monkey plasma, monkey cerebrospinal fluid, and human urine. In a method using gas chromatography followed by mass spectrometry, salvinorin A could be identified in human plasma, urine, saliva, and sweat at concentrations between 15$5000 \mathrm{ng} / \mathrm{mL}$ for plasma, urine and saliva, and 10-50 ng per patch for sweat. In human smokers of salvinorin A, this method was able to detect salvinorin A in urine and saliva (Pichini et al. 2005). In the leaves of Salvia divinorum, salvinorin A and other salvinorins and divinatorins were identified using liquid chromatography/electrospray ionisation multistage mass spectrometry (Medana et al. 2006). Another study used thin layer chromatography and gas chromatography/mass spectrometry to identify Salvia divinorum from 13 other Salvia species and from Cannabis sativa L (Jermain and Evans 2009). Futher use of thin layer chromatography was utilised along with desorption electrospray ionisation mass spectrometry to identify salvinorin A and other salvinorins in leaf material (Kennedy and Wiseman 2010). 
The first full synthesis of salvinorin A was published in 2007 (Scheerer et al. 2007) and took 29 steps to produce salvinorin A. Since then, several studies have been carried out to decrease the number of steps and reduce the cost of synthesis of salvinorin A, with one study requiring only 20 steps (Nozawa et al. 2008) and a more recent study by the same group taking only 13 (Hagiwara et al. 2009).

The binding site of salvinorin A on the KOPr has been investigated, and is of particular interest because of its radically different structure compared to classic KOPr agonists. A number of different amino acid residues have been shown to stabilise salvinorin $A$ in the putative binding pocket, including Gln115 (Kane et al. 2006; Kane et al. 2008), Tyr119 (Kane et al. 2006; Kane et al. 2008), Tyr313 (Yan et al. 2005; Kane et al. 2006; Kane et al. 2008), Ile316 (Kane et al. 2008) and Tyr320 (Yan et al. 2005; Kane et al. 2006; Kane et al. 2008) in helix 7. In helix 2, an important residues is Tyr119 (Yan et al. 2005; Kane et al. 2006). Ile316 was found to be particularly crucial for salvinorin A binding to KOPr (Kane et al. 2008). A number of residues are also necessary for activation of KOPr by salvinorin A, including Tyr312 (Yan et al. 2005), Tyr313 (Yan et al. 2005) and Tyr320 (Yan et al. 2005) from helix 7 and Tyr139 (Yan et al. 2005) from helix three.

\section{$\underline{\text { 1.5.1 Analogues of salvinorin A }}$}

Analogues of salvinorin A have been synthesized by altering a number of different residues of the compound. These alterations have not only highlighted important domains for binding and specificity but also produced analogues with increased affinity and prolonged half-lives. These studies have identified the importance of the furan ring, C2 carbon, C4 carbon, and C12 carbon for affinity and specificity to KOPr (Fig. 1.5). The furan ring is critical for KOPr affinity (Harding et al. 2006; Beguin et al. 2009); whereas, alterations in the $\mathrm{C} 2$ carbon lead to increased stability and changes in affinity (Chavkin et al. 2004; Beguin et al. 2005; Beguin et al. 2006; Bikbulatov et al. 2007), with the beta isomer binding better then the alpha isomer (Lee et al. 2010). Studies of the $\mathrm{C} 4$ carbon side chain have shown that long or bulky side chains lead to decreased affinity for KOPr (Lee et al. 2006), and alterations at the C12 carbon lead to decreased affinity for all opioid receptor isotypes (Yang et al. 2009). One salvinorin A analogue, herkinorin, has been generated that has selective affinity for the MOPr (Holden et al. 2007), and other compounds have been produced with affinity for both the KOPr and MOPr (Bikbulatov et al. 2008). Another analogue that has an N-methylacetamide at the 
C2 carbon shows KOPr selectivity, high affinity, slower metabolic transformation and oral efficacy, showing promise therefore as a potential therapeutic (Beguin et al. 2008). Modification of the $\mathrm{C} 1$ ketone creates weak KOPr antagonists, or KOPr agonists that show antagonism of the MOPr and DOPr (Munro et al. 2005; Holden et al. 2007). These compounds, with affinities for more than one receptor, may have important therapeutic potential, since simultaneous activation of the MOPr and DOPr might, along with the primary effect of the KOPr, decrease some of the unwanted side effects of the KOPr and may lead to better therapies.

The two salvinorin A derived compounds that we currently have access to, DS-1-240, and MOMSalB (DS-3-216), both have modified C2 regions, as shown in Figure 1.5. DS-1-240 shows KOPr selectivity with an $\mathrm{EC}_{50}$ value lower then salvinorin $\mathrm{A}$ and a longer half-life (Harding et al. 2005). DS-3-216 has been studied quite extensively and shows a higher affinity, efficacy, and longer half-life then salvinorin A in animal studies (Wang et al. 2008; Inan et al. 2009). DS-3-216 also induces ERK1/2 phosphorylation and primary astrocyte proliferation, but only induces the early phase ERK1/2 activation in an immortalised cell line, rather than both the early and late phase as seen with U69,593 (McLennan et al. 2008). Both of these compounds have also shown antiaddictive properties in a rat model of cocaine addiction (A. Morani, unpublished data). These compounds therefore would be interesting to study further to see how they modulate DAT in both cell systems and in isolated tissue. Due to the large potential for altering the structure of salvinorin A to make analogues, and the effects that these analogues have, derivatisation of salvinorin A has led to a novel chemical class of other KOPr agonists and possibly antagonists that may not possess the classical side effects of KOPr ligands and may be more suitable for the clinic. 


\subsection{Aims and Objectives}

The overall aim of the present study is to investigate the effects of the novel KOPr agonist salvinorin A, and two of its analogues, DS-1-240 and DS-3-216, on DAT in both tissue and cell models. This study investigated how these compounds act on DAT and if their effects are different from classic agonists. By investigating the effects of these compounds and comparing them to classic agonists it may be possible to identify differences in their mode of action. This may lead to greater understanding about how these compounds work, and possibly aid the development of novel pharmacotherapies for drug addiction.

The specific objectives of the study were (1) to investigate the effect of the novel KOPr agonists on DAT function in isolated rat brain tissue; (2) to investigate the effects of the novel KOPr agonists on DAT function in a cell model, and whether any changes observed occur via increased cell surface expression of DAT; (3) to investigate if the ERK1/2 pathway and lipid rafts are involved in the interaction between KOPr and DAT and (4) to establish rotating disk electrode voltammetry techniques to measure SERT function so that the effect of KOPr activation on SERT could be measured in rat brain tissue. 


\section{Chapter 2: General methods}

\subsection{Tissue culture}

All tissue culture work was carried out in a sterile Email Air Handling Class II Biological safety cabinet (AES Environment Pty LTD, Auburn, Australia). Sterile plasticware was purchased from BD Biosciences and Greiner Bio One. Cells were grown in a Heracell incubator (Kendro Laboratory Products, GmbH, Germany) under humid conditions at $37^{\circ} \mathrm{C}$ with $5 \% \mathrm{CO}_{2}$. For this study, three different cell lines were used: human embryonic kidney cells (HEK-293), green monkey kidney cells (COS7), and mouse neuroblastoma cells (N2A). All cell lines were purchased from American Type Culture Collection (ATCC, Manassas, VA, USA) and were used between passage number seven and 50 .

\subsubsection{Cell lines used in this study}

\subsubsection{Human embryonic kidney cells (HEK-293)}

HEK-293 are adherent immortalised human embryonic kidney cells that are transformed with adenovirus 5. They were cultured in Dulbecco's Modified Eagle's Medium (DMEM) high glucose medium (Gibco, Invitrogen, Auckland, NZ) containing 10\% fetal calf serum (FCS, ICP biologicals, Auckland, NZ), $200 \mu \mathrm{M}$ L-glutamine (Gibco, Invitrogen), and 1\% penstrep antibiotic (Penicillin G sodium 5000 units $/ \mathrm{mL}$, streptomycin sulphate 5000 units $/ \mathrm{mL}$ in $0.85 \%$ saline, Gibco, Invitrogen). Nonpenstrep medium was made from DMEM containing $200 \mu \mathrm{M}$ L-glutamine and $10 \%$ FCS and was used for cell transfections. For confocal microscopy, biotinylation, and Western blotting experiments, cells were plated at a density of $5 \times 10^{5}$ cells in $1 \mathrm{~mL}$ in $35 \mathrm{~mm}$ dishes. For rotating disk electrode voltammetry experiments (RDEV) cells were plated at a density of $3.5 \times 10^{6}$ cells in $7 \mathrm{~mL}$ in $100 \mathrm{~mm}$ dishes.

\subsubsection{Green monkey kidney cells (COS7)}

COS7 are adherent kidney cells from the African monkey Cercopithecus aethiops. transformed with SV40. These cells were cultured in DMEM high glucose medium containing $10 \%$ FCS, $200 \mu \mathrm{M}$ L-glutamine and 1\% penstrep. Non-penstrep medium was made from DMEM containing $200 \mu \mathrm{M}$ L-glutamine and 10\% FCS and was used for cell transfections. Cells were plated for confocal experiments at a density of $3 \times 10^{5}$ cells/ in $1 \mathrm{~mL}$ in $35 \mathrm{~mm}$ dishes. 


\subsubsection{Mouse neuroblastoma cells (N2A)}

N2A are adherent mouse brain neuroblastoma cells from Mus musculus. They were cultured in Roswell Park Memorial Institute (RPMI) 1640 medium containing 10\% FCS and $1 \%$ penstrep antibiotic. Non-penstrep media contained RPMI with $10 \%$ FCS. Cells were plated for confocal microscopy experiments at a density of $1 \times 10^{6}$ cells in $1 \mathrm{~mL}$ in $35 \mathrm{~mm}$ dishes.

\subsubsection{Cell growth and passaging}

Cell stocks were stored in liquid nitrogen and were thawed for use in experiments. To defrost cells, a vial was removed from the liquid nitrogen dewer and defrosted in a $37^{\circ} \mathrm{C}$ waterbath. Cells were immediately added to a $15 \mathrm{~mL}$ Falcon tube containing $10 \mathrm{~mL}$ of their respective culture medium and centrifuged at $300 \mathrm{~g}$ for $5 \mathrm{~min}$. The cells were then resuspended in $7 \mathrm{~mL}$ of culture medium and added to a $75 \mathrm{~mL}$ flask to grow. The following day, cells were fed by removal of old culture medium and the addition of fresh medium. When the cells had reached $80 \%$ confluency they were passaged into a $250 \mathrm{~mL}$ flask. Once these cells had reached $80 \%$ confluency they were passaged into two separate $250 \mathrm{~mL}$ flasks, one for use in experiments and the other for freezing down to replace stocks removed from the liquid nitrogen.

Cells were passaged when they were $80-90 \%$ confluent. Cells were washed twice with $5 \mathrm{~mL}$ of sterile $1 \mathrm{x}$ phosphate buffered saline (PBS, $137 \mathrm{mM} \mathrm{NaCl}, 2.7 \mathrm{mM} \mathrm{KCl}, 8.1$ $\mathrm{mM} \mathrm{Na}_{2} \mathrm{HPO}_{4}, 1.5 \mathrm{mM} \mathrm{KH}{ }_{2} \mathrm{PO}_{4}, \mathrm{pH}$ 7.4) (Appendix 1) and then incubated at $37^{\circ} \mathrm{C}$ in $1.5 \mathrm{~mL}$ of TrpLE Express (Gibco, Invitrogen) for $5 \mathrm{~min}$. When cells had detached they were removed by washing with medium, transferred into a sterile $15 \mathrm{~mL}$ Falcon tube and centrifuged at $300 \mathrm{~g}$ for $5 \mathrm{~min}$. The supernatant was poured off and cells resuspended in culture medium. The concentration of live cells was then determined by counting using a haemocytomter and $0.4 \%$ trypan blue (Sigma). To continue the cell line, cells were diluted 1:4 and reseeded in $250 \mathrm{~mL}$ flasks. For confocal microscopy, 1 $\mathrm{mL}$ of cells was plated on to a $35 \mathrm{~mm}$ glass bottom dishes (Mattec, Loveland, $\mathrm{OH}$, USA), and for cell surface biotinylation and Western blotting experiments, $1 \mathrm{~mL}$ of cells was plated on $35 \mathrm{~mm}$ plastic petri dishes.

To freeze cells, cells in log growth phase were passaged and resuspended to $1 \times 10^{6}$ cells/mL in their respective culture medium containing $10 \%$ dimethyl sulfoxide 
(DMSO). Aliquots of $1 \mathrm{~mL}$ were frozen in cryovials overnight at $-80^{\circ} \mathrm{C}$ and then transferred to liquid nitrogen.

\subsubsection{Cell transfection}

Cells were transfected the day after plating, when cells were $60-70 \%$ confluent. Cells were transfected using Lipofectamine 2000 reagent (Invitrogen) and Optimum 1 medium (Invitrogen). For HEK-293 cells, $3 \mu \mathrm{L}$ of Lipofectamine 2000 was made up to $50 \mu \mathrm{L}$ with Optimum 1 and incubated for $5 \mathrm{~min}$. In a separate tube, myc-tagged rat kappa opioid receptor (myc-rKOPr, kindly donated by L. Devi) plasmid DNA and yellow fluorescent protein-tagged human dopamine transporter (YFP-hDAT, kindly donated by J. Javitch) plasmid DNA were made up to $50 \mu \mathrm{L}$ with Optimum 1, to a final concentration of $0.6 \mu \mathrm{g} / \mathrm{mL}$ and $0.4 \mu \mathrm{g} / \mathrm{mL}$ respectively. After the $5 \mathrm{~min}$ incubation, the 2 tubes were mixed and incubated at room temperature for $20 \mathrm{~min}$. Cells that were to be transfected were fed with $1 \mathrm{~mL}$ of penstrep-free medium, and $100 \mu \mathrm{L}$ of the transfection mixture was added. For RDEV experiments cells were transfected following the above protocol with $1 \mu \mathrm{g} / \mathrm{mL}$ of green fluorescent protein-tagged human serotonin transporter (GFP-hSERT, kindly donated by S. Ramamoorthy). Cells were used $48 \mathrm{~h}$ after transfections. For COS7 and N2A cells, $4 \mu \mathrm{L}$ of Lipofectamine 2000 was used, and 1.2 $\mu \mathrm{g} / \mathrm{mL}$ of myc-rKOPr and $0.8 \mu \mathrm{g} / \mathrm{mL}$ of YFP-hDAT DNA was used.

\subsection{Plasmid Preparation}

\subsubsection{Transformation of MAX Efficiency ${ }^{\circledR}$ DH5 $\alpha^{\mathrm{TM}}$ Competent Cells}

A $100 \mu \mathrm{L}$ aliquot of MAX Efficiency ${ }^{\circledR}$ DH5 $\alpha^{\mathrm{TM}}$ Competent Cells (Invitrogen) was thawed on wet ice and then divided into $50 \mu \mathrm{L}$ aliquots in ice-cold microcentrifuge tubes. To one of the tubes, 15 ng of plasmid DNA (Table 2.1) was added and incubated on ice for $1 \mathrm{~h}$. The other tube acted as a negative control. Both tubes were then heat shocked at $42^{\circ} \mathrm{C}$ for $2 \mathrm{~min}$ in a heat block (Global Science) and then placed on ice for a further $5 \mathrm{~min}$. After cooling, $450 \mu \mathrm{L}$ of room temperature SOC medium (Invitrogen) was added to each of the tubes, and the tubes were placed in a $37^{\circ} \mathrm{C}$ Shaker (Bioline, Edwards Instrument Company, Australia) set at $225 \mathrm{rpm}$ for $1 \mathrm{~h}$. Following this, 100 $\mu \mathrm{L}$ of each culture was spread onto a Luira Broth (LB) agar plate $(10 \mathrm{~g} / \mathrm{L} \mathrm{NaCl}, 5 \mathrm{~g} / \mathrm{L}$ Yeast extract, $10 \mathrm{~g} / \mathrm{L}$ Yeast agar, $10 \mathrm{~g} / \mathrm{L}$ Bactotryptone) (Appendix 1) containing either $100 \mu \mathrm{g} / \mathrm{mL}$ ampicillin or $50 \mu \mathrm{g} / \mathrm{mL}$ kanamycin to create a 'lawn' culture. The plates were incubated overnight in a $37^{\circ} \mathrm{C}$ humid incubator (Bioline), and the following day the procedure below was carried out. 
Table 2.1 Plasmid information

\begin{tabular}{|l|l|l|l|}
\hline Gene & Vector & Resistance & Provided by \\
\hline myc-rKOPr & pcDNA3 & Ampicillin $(100 \mu \mathrm{g} / \mathrm{mL})$ & L. Devi (Jordan and Devi 1999) \\
\hline YFP-hDAT & pciHyg & Ampicillin $(100 \mu \mathrm{g} / \mathrm{mL})$ & J. Javitch (Daws et al. 2002) \\
\hline GFP-hSERT & peGFP- & Kanamycin $(50 \mu \mathrm{g} / \mathrm{mL})$ & $\begin{array}{l}\text { S. Ramamoorthy (Scholze et al. } \\
2000)\end{array}$ \\
& C1 & & \\
\hline
\end{tabular}

\subsubsection{Escherichia coli cultivation}

To cultivate single colonies, a scraping of the transformation plate was taken and streaked onto LB agar plates containing the appropriate antibiotic and incubated overnight at $37^{\circ} \mathrm{C}$ with humidity. This was only carried out if the negative control plate showed no growth since growth on this control indicated contamination of the bacterial cells and a new transformation was then conducted. The following day, individual colonies were removed from the plate using a sterile pipette tip and added to $2 \mathrm{~mL}$ of LB Broth (10 g/L NaCl, $5 \mathrm{~g} / \mathrm{L}$ Yeast extract, $10 \mathrm{~g} / \mathrm{L}$ Bactotryptone) (Appendix 1) containing the appropriate antibiotic. This culture was incubated for $24 \mathrm{~h}$ in a shaking, humid $37^{\circ} \mathrm{C}$ incubator (Bioline). After $24 \mathrm{~h}, 200 \mu \mathrm{L}$ of bacteria was removed and added to $200 \mathrm{~mL}$ of LB broth containing the appropriate antibiotic, and this culture was incubated for another $24 \mathrm{~h}$ in a shaking, humid $37^{\circ} \mathrm{C}$ incubator. After $24 \mathrm{~h}$ the plasmid preparation (Section 2.2) was carried out.

\subsubsection{Plasmid purification using the Qiagen HiSpeed Maxi Kit}

Plasmid DNA was purified from $200 \mathrm{~mL}$ bacterial culture following the manufacturer's protocol.

Briefly, the $200 \mathrm{~mL}$ bacterial culture was centrifuged at $6,000 \mathrm{~g}$ for $25 \mathrm{~min}$ at $4^{\circ} \mathrm{C}$ in a Sorvall Evolution RC centrifuge (Kendro Laboratory Products, Bath, UK) and the supernatant discarded. The bacterial pellet was resuspended in $10 \mathrm{~mL}$ of resuspension buffer containing RNase A (Buffer P1). To lyse the cells, the resuspended E. coli was transferred to a sterile $50 \mathrm{~mL}$ tube, and $10 \mathrm{~mL}$ of lysis buffer (Buffer P2) was added and mixed by inverting 4-6 times. The solution was incubated for $5 \mathrm{~min}$ at room temperature then $10 \mathrm{~mL}$ of chilled neutralisation buffer (Buffer P3) was added to stop cell lysing and mixed by inverting the tube 4-6 times. This lysate was then transferred into the QIAfilter Cartridge and incubated for $10 \mathrm{~min}$ at room temperature. During this 
time a HiSpeed Maxi Tip was placed over a waste container and equilibrated by the application of $10 \mathrm{~mL}$ of Buffer QBT through the filter. The lysate was then filtered through the HiSpeed Maxi Tip into a waste container, trapping the DNA within the tip. The tip was then washed with $60 \mathrm{~mL}$ of wash buffer (Buffer QC), and then the DNA was eluted into a sterile $50 \mathrm{~mL}$ tube using $15 \mathrm{~mL}$ of elution buffer (Buffer QF). The DNA was precipitated by incubation with $10.5 \mathrm{~mL}$ of room temperature molecular grade isopropanol (Sigma) for $5 \mathrm{~min}$. The isopropanol mixture was transferred into a 30 $\mathrm{mL}$ syringe attached to a QIAprecipitator Maxi Module and filtered through the precipitator into a waste container. The DNA remained trapped in the filter. The precipitator filter was then rapidly washed with $2 \mathrm{~mL}$ of $70 \%$ ethanol (Ajax Chemicals, Auckland, NZ) via the syringe. The filter was then air dried by quickly pushing air via the syringe through the filter twice, and then the filter was dried on a paper towel to prevent ethanol carryover. The precipitation filter was then attached to a $5 \mathrm{~mL}$ syringe and placed over a microcentrifuge tube, $500 \mu \mathrm{L}$ of endotoxin free buffer (Buffer TE) was added to the syringe and the DNA was eluted into the microcentrifuge tube. The eluted DNA was then transferred back into the syringe and filtered a second time through the precipitator into the same tube.

The yield of DNA was determined using a Nanodrop ND-1000 spectrophotometer (BioLab, Auckland, NZ). The Nanodrop was initialised and then blanked using $1 \mu \mathrm{L}$ of double distilled water. A $1 \mu \mathrm{L}$ sample of eluted DNA was placed onto the sensor and the amount of DNA present was measured at $230 \mathrm{nM}$. Yields of above $500 \mathrm{ng} / \mu \mathrm{L}$ with a 260/280 ratio of 1.6-2.0 were deemed acceptable and used for transfections.

If yields of $1 \mathrm{mg} / \mu \mathrm{L}$ or above were purified, the $2 \mathrm{~mL}$ culture of bacteria was frozen in $500 \mu \mathrm{L}$ aliquots in $20 \%$ glycerol at $-80^{\circ} \mathrm{C}$ for future use so that transformations were not necessary each time plasmid was needed. In this case, a scraping from the frozen bacteria stock was streaked onto a LB agar plate containing the appropriate antibiotic to obtain a single colony that could then be cultured in $2 \mathrm{~mL}$ of LB broth containing the correct antibiotic. 


\subsection{Cell surface biotinylation}

HEK-293 cells were plated and transfected as described in sections 2.1.2 and 2.1.3, and cell surface biotinylation was carried out $48 \mathrm{~h}$ after transfection. Methods were carried out as previously described (Bolan et al. 2007; Zapata et al. 2007).

KOPr agonists were dissolved in DMSO at a stock concentration of $75 \mathrm{mM}$ and diluted further in KREBS to a working concentration of $210 \mu \mathrm{M}$. From this working stock, 50 $\mu \mathrm{L}$ of compound was added to $1 \mathrm{~mL}$ of medium in the cell culture dishes to give a final concentration of $10 \mu \mathrm{M}$. Compounds were added to the dishes, and incubated for either the time required for maximal effect to occur, as determined by confocal microscopy experiments, or for $30 \mathrm{~min}$. Cells were then washed twice with ice-cold $\mathrm{PBS} / \mathrm{Ca}-\mathrm{Mg}$ (PBS containing $0.1 \mathrm{mM} \mathrm{CaCl}_{2}$ and $1 \mathrm{mM} \mathrm{MgCl}_{2}, \mathrm{pH}$ 8.0) (Appendix 1), and incubated for $30 \mathrm{~min}$ with $1 \mathrm{mg} / \mathrm{mL}$ of EZ-link NHS-Sulfo-SS-biotin (Pierce) in ice-cold PBS/Ca$\mathrm{Mg}$ in the dark at $4{ }^{\circ} \mathrm{C}$ with shaking. Cells were then washed twice with ice-cold 100 $\mathrm{mM}$ glycine in PBS/Ca-Mg, and then incubated for $20 \mathrm{~min}$ with $100 \mathrm{mM}$ glycine in PBS/Ca-Mg in the dark at $4{ }^{\circ} \mathrm{C}$ with shaking. Cells were lysed in $400 \mu \mathrm{L}$ of RIPA buffer (10 mM Tris-HCl, $150 \mathrm{mM} \mathrm{NaCl}, 1 \mathrm{mM}$ EDTA, 1\% Triton-X-100, 0.1\% SDS, $1 \%$ sodium deoxycholate, $\mathrm{pH} 7.5$, (Appendix 1)) containing $10 \mu \mathrm{L} / \mathrm{mL}$ of protease inhibitor (Sigma) for $1 \mathrm{~h}$ in the dark at $4{ }^{\circ} \mathrm{C}$ with shaking. Lysates were transferred to microcentrifuge tubes and centrifuged at 16,000 $\mathrm{g}$ in a benchtop centrifuge (Eppendorf, Eppendorf South Pacific, Australia) for $30 \mathrm{~min}$ at $4{ }^{\circ} \mathrm{C}$. A $50 \mu \mathrm{L}$ sample of the supernatant was removed for the total fraction, and kept on ice and in the dark until needed. Then $400 \mu \mathrm{L}$ of the supernatant was applied to filter columns containing 100 $\mu \mathrm{L}$ of streptavidin beads (Pierce) and incubated for $1 \mathrm{~h}$ at room temperature with gentle mixing. The plugs were removed from the columns, and the columns were spun down at $4{ }^{\circ} \mathrm{C}$ for $1 \mathrm{~min}$ at $500 \mathrm{~g}$. A $50 \mu \mathrm{L}$ sample of the spun down liquid was then removed for the internal fraction and kept on ice in the dark until needed. The columns were then washed $3 \mathrm{x}$ with $400 \mu \mathrm{L}$ of RIPA buffer by spinning at 16,000 g for $1 \mathrm{~min}$. Plugs were replaced in the columns, and the bound proteins were eluted with $40 \mu \mathrm{L}$ of $5 \mathrm{x}$ reducing buffer (62.5 mM Tris-HCl, pH 6.8, 2\% SDS, 20\% glycerol, 1\% bromophenol blue (Appendix 1)) containing 10\% $\beta$-mercaptoethanol (Sigma) for $30 \mathrm{~min}$ with gentle mixing. The plugs were then removed from the columns, and the columns were placed into microcentrifuge tubes and centrifuged at $4{ }^{\circ} \mathrm{C}$ for $1 \mathrm{~min}$ at $500 \mathrm{~g}$ to retrieve the cell surface fraction. Five times reducing buffer containing 10\% $\quad \beta$-mercaptoethanol (15 $\mu \mathrm{L}$ ) was then added to the total and internal fractions and $20 \mu \mathrm{L}$ of the samples were 
loaded onto $10 \%$ acrylamide gels and electrophoresed, then transferred to Western blotting membranes as described in section 2.4. Using Western blotting, GAPDH immunoblotting was carried out as a control to ensure cells were not lysed before biotin was added, as GAPDH is only found intracellularly in HEK-293 cells.

DAT expression was measured by the intensity of the YFP tag in the polyacrylamide gels prior to Western transfer and was analyzed using Image-J software. The integrated density of the bands from each lane was measured as one using the analyze measure, integrated density command. Background readings were taken and subtracted from the sample bands. The background corrected internal and cell surface bands were then normalized to the background corrected total protein bands, giving an arbitrary number. The normalised internal and cell surface numbers were then normalised to the vehicletreated samples for each gel so that between-gel comparisons could be made. The numbers were graphed using Graphpad Prism 4 software (GraphPad Software Inc, La Jolla, CA, USA) and a one-way ANOVA, with a Bonferroni post test, was conducted to test for significance between treatment groups.

\subsection{SDS polyacrlyamide gel electrophoresis and Western blotting}

Protein samples from experiments were run on 10- or 15-well SDS-PAGE gels with a $10 \%$ separating gel and a $4 \%$ stacking gel (Appendix 1). Gels were made the day before the experiment and stored wrapped in double distilled soaked paper towels at $4^{\circ} \mathrm{C}$. Gels were run for $90 \mathrm{~min}$ at $120 \mathrm{~V}$ in $1 \mathrm{x}$ running buffer $(3.5 \mathrm{mM}$ SDS, $25 \mathrm{mM}$ Tris base, $190 \mathrm{mM}$ glycine) (Appendix 1), or until the blue dye front had run off the gel. After electrophoresis, the gels were removed from the electrophoresis set up and placed in containers containing distilled water, and YFP-hDAT was visualised with a Fuji film laser scanner (Fujifilm FLA-5100, Tokyo, Japan) using a $473 \mathrm{~nm}$ laser and BPB1 filter set at $800 \mathrm{~V}$.

After scanning, proteins were transferred onto a polyvinylidine difluoride (PVDF) (0.2 $\mu \mathrm{M}$ pore size, Millipore Corporation, MA) membrane. The membrane was pre-soaked in methanol for $1 \mathrm{~min}$ followed by transfer buffer (190 mM glycine, $25 \mathrm{mM}$ Tris, $20 \%$ methanol) (Appendix 1) for $5 \mathrm{~min}$ before being used for transfer. The transfer cassette was assembled (from back to front - black side of cassette, sponge, 3 MM filter paper, gel, membrane, $3 \mathrm{MM}$ filter paper, sponge, clear side of cassette) and inserted into the 
Bio-Rad Tetracell transfer set (Bio-Rad) containing an ice-pack, and the tank was filled with transfer buffer. The transfer was run at $20 \mathrm{~V}$ for $17 \mathrm{~h}$ at $4{ }^{\circ} \mathrm{C}$.

The following day, the membranes were washed for 5 min in $1 \mathrm{x}$ Tris buffered saline (TBS, $50 \mathrm{mM}$ Tris base, $150 \mathrm{mM} \mathrm{NaCl}, \mathrm{pH}$ 7.5) (Appendix 1), blocked for $1 \mathrm{~h}$ in $5 \%$ bovine serum albumin (BSA) in TBS containing $0.1 \%$ Tween 20 (T-TBS) or $10 \%$ milk powder in T-TBS (Appendix 1) at room temperature on a rocker, and then the appropriate antibodies were applied as detailed in Table 2.2. The primary antibody was diluted in the appropriate blocking buffer, and membranes were incubated for either $1 \mathrm{~h}$ at room temperature or overnight at $4^{\circ} \mathrm{C}$ on a rocker. After the incubation with the primary antibody, membranes were quickly washed $3 \mathrm{x}$ in T-TBS and then washed $3 \mathrm{x}$ for $5 \mathrm{~min}$ in T-TBS. The secondary antibody was then added in T-TBS and incubated for $1 \mathrm{~h}$ in the dark at room temperature with gentle rocking. The membranes were then washed $3 \mathrm{x}$ in T-TBS and then $3 \mathrm{x}$ in TBS and read on a Fujifilm FLA5100 fluorescent scanner (Fujifilm, Tokyo, Japan). Secondary antibodies labelled with Cy5 were read using a $635 \mathrm{~nm}$ laser and DBR1 filter and secondary antibodies labelled with Cy3 were read using a $532 \mathrm{~nm}$ laser with a DGR1 filter.

ImageJ software was used to analyse data. The integrated densities of the YFP-hDAT and immunoblotted proteins had the average background subtracted and then the density values were normalised to the control so that results could be compared between gels or membranes. These numbers were graphed using GraphPad Prism 4 software, and statistical analysis was carried out. 
Table 2.2: Antibodies used for Western blotting

\begin{tabular}{|c|c|c|c|c|}
\hline Antibody & $\begin{array}{l}\text { Blocking solution } \\
\text { used }\end{array}$ & $\begin{array}{l}\text { Dilution } \\
\text { factor }\end{array}$ & $\begin{array}{l}\text { Incubation } \\
\text { time }\end{array}$ & $\begin{array}{l}\text { Secondary } \\
\text { antibody }\end{array}$ \\
\hline $\begin{array}{l}\text { Mouse monoclonal anti- } \\
\text { GAPDH (Abcam, } \\
\text { supplied by Sapphire } \\
\text { Bioscience, Hamilton } \\
\text { NZ) }\end{array}$ & $5 \%$ BSA in T-TBS & $1: 6,000$ & $1 \mathrm{~h}$ & $\begin{array}{l}\text { Anti-mouse Cy5 } \\
(1: 5,000) \\
\text { (Amersham, } \\
\text { supplied by } \\
\text { Global Science) }\end{array}$ \\
\hline $\begin{array}{l}\text { Rabbit polyclonal anti- } \\
\text { tubulin } \\
\text { (Abcam) }\end{array}$ & $\begin{array}{l}10 \% \text { milk powder } \\
\text { in T-TBS }\end{array}$ & $1: 5,000$ & $1 \mathrm{~h}$ & $\begin{array}{l}\text { Anti-rabbit Cy3 } \\
(1: 5,000) \\
\text { (Amersham) }\end{array}$ \\
\hline $\begin{array}{l}\text { Mouse monoclonal anti- } \\
\text { P-ERK42/44 (P-ERK1/2) } \\
\text { (Santa Cruz supplied by } \\
\text { Thermofisher, Auckland, } \\
\text { NZ) }\end{array}$ & $5 \% \mathrm{BSA}$ in $\mathrm{T}-\mathrm{TBS}$ & $1: 500$ & overnight & $\begin{array}{l}\text { Anti-mouse Cy5 } \\
(1: 5,000) \\
\text { (Amersham) }\end{array}$ \\
\hline $\begin{array}{l}\text { Rabbit monoclonal } \\
\text { ERK42/44 (ERK1/2) } \\
\text { (Cell Signalling supplied } \\
\text { by Thermofisher) }\end{array}$ & $5 \%$ BSA in T-TBS & $1: 1,000$ & overnight & $\begin{array}{l}\text { Anti-rabbit Cy3 } \\
(1: 5,000) \\
\text { (Amersham) }\end{array}$ \\
\hline
\end{tabular}




\section{5 trans-4-[4-(dimethylamino)styryl]-1-methylpyridinium (ASP $\left.{ }^{+}\right)$uptake}

HEK-293 cells were plated and transfected for confocal microscopy as described in 2.1.2 and 2.1.3 and used $48 \mathrm{~h}$ after transfection.

\section{$\underline{\text { 2.5.1 Acute } \mathrm{ASP}^{+} \text {uptake studies }}$}

Methods were carried out as previously described (Schwartz et al. 2003; Bolan et al. 2007). The fluorescent monoamine transporter substrate 4-(4-(dimethylamino)-styryl)$\mathrm{N}$-methylpyridinium (ASP ${ }^{+}$Tocris) was made into a $10 \mathrm{mM}$ stock solution in sterile KREBS buffer (130 mM NaCl, $1.3 \mathrm{mM} \mathrm{KCl,} 2.2 \mathrm{mM} \mathrm{CaCl}_{2}, 1.2 \mathrm{mM} \mathrm{MgSO}_{4} .6 \mathrm{H}_{2} 0,1.2$ $\mathrm{mM} \mathrm{KH}{ }_{2} \mathrm{PO}_{4}, 10 \mathrm{mM}$ HEPES, $10 \mathrm{mM}$ D-glucose, $\mathrm{pH}$ 7.4) (Appendix 1). This stock was diluted to a $10 \mu \mathrm{M}$ working concentration in KREBS buffer and placed in a $37^{\circ} \mathrm{C}$ water bath with KREBS buffer. An Olympus Fluoview FV1000 confocal laser scanning biological microscope (inverted model 1X81) was used to monitor $\mathrm{ASP}^{+}$ uptake and binding of cells, using a $40 \mathrm{X}$ water immersion objective $(\mathrm{NA}=0.9)$. To maintain the temperature of the cells at $37^{\circ} \mathrm{C}$ during confocal microscopy, a heat-plated, insulated, double-layer heating chamber (INU-21LCS-F1 model, Tokai Hit Co., Ltd, Japan) mounted on the microscope stage was filled with distilled water and allowed to warm to $37^{\circ} \mathrm{C}$. $\mathrm{ASP}^{+}$fluorescence was measured using a $558 \mathrm{~nm}$ laser $\left(\mathrm{ASP}^{+}\right.$filter: 570 $\mathrm{nm}$ excitation; $670 \mathrm{~nm}$ emission) at settings of $1.7 \%$ power, high voltage (HV) $600 \mathrm{~V}$, offset $9 \%$, and gain 1\%. YFP fluorescence was measured using a $473 \mathrm{~nm}$ laser (YFP filter: $485 \mathrm{~nm}$ excitation; $545 \mathrm{~nm}$ emission) at settings of $0.7 \%$ power, HV $700 \mathrm{~V}$, offset $10 \%$, and gain $1 \%$. Differential interference contrast (DIC) was used to visualise the collection of cells. The microscope pinhole was opened to $500 \mu \mathrm{m}$ to allow for more light from a greater $\mathrm{z}$ plane to get through. This decreases confocality and allows $\mathrm{ASP}^{+}$ accumulation to be more representative of uptake from the whole cell rather than just a single plane. Agonists were made up to a stock concentration of $75 \mathrm{mM}$ in DMSO and then diluted in KREBS buffer to a working stock of $1.01 \mathrm{mM}$ so that when $10 \mu \mathrm{L}$ was added to the dish of cells, the final concentrations of the agonists were $10 \mu \mathrm{M}$. The working stock was further diluted for experiments where $5 \mu \mathrm{M}$ and $1 \mu \mathrm{M}$ were required. Control experiments were carried out using DMSO-matched solutions. Images were acquired using Olympus FV10-ASW software.

Acute $\mathrm{ASP}^{+}$experiments were conducted as previously described (Bolan et al. 2007; Zapata et al. 2007). Culture medium was removed from the $35 \mathrm{~mm}$ glass bottom petri dish by aspiration and replaced with KREBS buffer at $37^{\circ} \mathrm{C}$. Dishes were mounted on 
the microscope stage, and the microscope was focused on the cell layer. Once an appropriate area of cells was located, KREBS buffer was removed and the time course started (120 scans, every $5 \mathrm{~s}$ ). Once the first image was collected, $\mathrm{ASP}^{+}$at $37^{\circ} \mathrm{C}$ was added. At $250 \mathrm{~s}$ (DS-1-240, DS3-214, and U50,488H) or $300 \mathrm{~s}$ (Salvinorin A) after $\mathrm{ASP}^{+}$was added, $10 \mu \mathrm{L}$ of the agonist was added. For experiments in which the KOPr antagonist norbinaltorphimine (nor-BNI in sterile KREBS buffer, Sigma) was used, dishes were incubated with $1 \mu \mathrm{M}$ nor-BNI 30 min prior to the addition of $\mathrm{ASP}^{+}$. For experiments in which pertussis toxin (PTX in sterile PBS, Sapphire Bioscience) was used, dishes were incubated with $100 \mathrm{ng} / \mathrm{ml}$ of PTX for $16-24 \mathrm{~h}$ before the $\mathrm{ASP}^{+}$was added.

\section{$\underline{2.5 .230 \mathrm{~min} \mathrm{ASP}^{+} \text {studies }}$}

For 30 min $\mathrm{ASP}^{+}$experiments, the agonist or an equal volume of DMSO (control) was added to the cells in their culture medium at a final concentration of $10 \mu \mathrm{M}$, and cells were returned to the incubator for $30 \mathrm{~min}$. After the $30 \mathrm{~min}$ incubation, culture medium was replaced with fresh KREBS buffer containing the agonist, and an appropriate area of cells was chosen for measuring uptake. The same microscope settings were used as for the acute $\mathrm{ASP}^{+}$uptake experiments except that a time course of 70 images, $5 \mathrm{~s}$ apart was used. The KREBS buffer was removed and the time course started. After the first image was collected, $\mathrm{ASP}^{+}$containing the agonist was added and the subsequent uptakes were measured.

\subsection{3 $\mathrm{ASP}^{+}$binding studies}

A single image was taken of the cells used in the $\mathrm{ASP}^{+}$uptake experiments using the DIC, YFP and $\mathrm{ASP}^{+}$filters (settings same as in section 2.5.1 above). The KREBS buffer was then removed, and $10 \mu \mathrm{M}$ of the agonist was added in fresh KREBS buffer. The cells were incubated for the time taken for the maximal effect to be reached as determined in the uptake studies (Section 4.3.1). A solution of $\mathrm{ASP}^{+}$containing the agonist was then added, and a time-course of 100 scans was started, imaging DIC and $\mathrm{ASP}^{+}$at maximum speed. This allowed the initial binding of $\mathrm{ASP}^{+}$to $\mathrm{DAT}$ to be measured. The slope of the line was analysed as described below.

\subsubsection{Data analysis for $\mathrm{ASP}^{+}$uptake and binding studies}

Cells expressing YFP-hDAT were manually outlined using Fluoview FV1000 software, and these regions of interest (ROI) were graphed over time to show the changes in $\mathrm{ASP}^{+}$ 
fluorescence. The data from these graphs were then transferred into a Microsoft Excel spreadsheet, and each cell's $\mathrm{ASP}^{+}$uptake was graphed individually. Cells that did not have linear $\mathrm{ASP}^{+}$uptake graphs were discarded as this indicated that the cells moved or drifted over the period of the experiment. The initial YFP fluorescence was also recorded so that cells could be selected based on their YFP-hDAT expression. Cells expressing less than 250 arbitrary fluorescence units (AFU) or more then 1000 AFU of YFP-hDAT were not used.

To analyse $\mathrm{ASP}^{+}$uptake data, a linear trendline was generated for a period of $60 \mathrm{~s}, 10 \mathrm{~s}$ before KOPr agonist addition, and compared to a linear trendline created for a period of $60 \mathrm{~s}$ after drug addition at the time of maximal change in uptake. The change in slope was then calculated as a percentage change ((slope after drug/slope before drug)x100) and graphed using Graphpad Prism. For 30 min agonist treatment experiments, the AFU at $300 \mathrm{~s}$ was taken and normalised to the YFP-hDAT expression from the first image. This normalised number was compared to DMSO-treated controls. For binding studies the change in binding was analysed using data from 3 to $7 \mathrm{~s}$ after $\mathrm{ASP}^{+}$addition. The slope of this line was normalized to the amount of YFP expressed on each cell, as seen from the initial scan at $\mathrm{t}=0$. Maximal change in uptake was determined by taking the slope of the 60 s-trendline 30 s, 60 s, 100 s, 150 s, 200 s, 250 s after agonist addition to find the time where the greatest change was seen.

GraphPad Prism was used to carry out one-way ANOVAs followed by Bonferroni Post tests on $\mathrm{ASP}^{+}$uptake and binding data.

\subsection{Total internal reflection fluorescence microscopy (TIRFM)}

TIRFM methods followed the protocol of Furman et al. (Furman et al. 2009a) (Appendix 2, see DVD for representative movie). HEK-293 cells were plated and transfected for confocal microscopy as described in section 2.1.2 and 2.1.3 and used 48 $\mathrm{h}$ after transfections. An Olympus Fluoview 2000 microscope and a QImaging Digital Camera 6.2.0.6 (QImaging ROLERA-MGI Fast1394; Surrey, BC, Canada) were used to conduct TIRFM. The cell heating chamber from section 2.5.1 was placed on the microscope stage and heated to $37^{\circ} \mathrm{C}$, and carbogen gas $\left(95 \% \mathrm{O}_{2}, 5 \% \mathrm{CO}_{2}\right)$ was gently blown through the cell chamber throughout the experiment to maintain optimal $\mathrm{pH}$. A $300 \mu \mathrm{L}$ syringe containing the compound to be tested was inserted into an inlet port of the cell chamber, and the dishes containing the transfected cells were placed in the cell 
chamber. Cells were focused on using the 100X objective lens (Olympus, PLAPO 100X, OTIRFM, NA 1.45), and cells were left to equilibrate in the enclosed cell chamber for $20 \mathrm{~min}$. A region of cells was then chosen using FITC epi-fluorescence, the TIRFM camera and Image Pro Plus 7.0 acquisition software (MediaCybernetics, Bethesda, MD, USA). The microscope was then set to TIRFM, with a penetration depth of $100 \mathrm{~nm}$, a FS of $11 \mathrm{~mm}$ and a refractive index of the sample of 1.52 and the microscope was focused on the cell membrane where it adhered to the glass-bottom dish. Cells were chosen that were expressing YFP-hDAT and were able to be clearly seen at a laser power of $0.5 \%$ (473 nm laser, YFP filter: $485 \mathrm{~nm}$ excitation; $545 \mathrm{~nm}$ emission). The camera (QImaging Digital Camera, 6.2.0.6) was set to an acquisition area of 512 x 512 pixels, with a capture depth of 14 bit mono (16 bit image class) and an exposure preview of $00.902 .580 \mathrm{~s}$ with a capture exposure time of $3 \mathrm{~s}$ using Image Pro Plus Software.

For short time-courses, images were captured every $5 \mathrm{~s}$ for 60 images. After 20 images, $50 \mu \mathrm{L}$ of agonist was added through the syringe. For 40 min time courses, the first image was captured at time 0 , and then images were captured every $1 \mathrm{~min}$ for $40 \mathrm{~min}$ (40 total scans). After $10 \mathrm{~min}, 50 \mu \mathrm{L}$ of KOPr agonist was added using the inlet port syringe so that the final concentration in the dish was $10 \mu \mathrm{M}$. For the $40 \mathrm{~min}$ experiment the laser had to be manually turned off and on between scans so that the samples were not photo-bleached over the $40 \mathrm{~min}$. Controls showed no significant photo-bleaching over either of these time-courses.

Images were analysed using Image J. Each cell was manually traced around at each scan time and the average pixel intensity was taken. These numbers were normalised to the first scan, and the normalised values were graphed in Graph Pad Prism. 


\section{Chapter 3: Effect of KOPr agonists on the function of DAT and SERT in minced brain tissue from the rat}

\subsection{Introduction}

The KOPr regulates DA concentrations in the synapse, with acute treatment leading to an overall decrease in DA, and chronic KOPr treatment appearing to cause an increase in DA in the synapse. This effect has been shown to be mediated both by a decrease in release of DA (Di Chiara and Imperato 1988b), and an increase in DAT function (Thompson et al. 2000). KOPr activation has also shown anti-addictive affects in animal models. Acute KOPr agonist treatment inhibits the locomotor-stimulated effects of cocaine (Collins et al. 2001a; Collins et al. 2001b) and prevents cocaine-primed reinstatement in rats (Schenk et al. 1999; Morani et al. 2009). Previous RDEV studies have shown that acute addition of KOPr agonists leads to increased function of DAT in rat NAc tissue (Thompson et al. 2000). An acute treatment following intraperitoneal injection $(0.32 \mathrm{mg} / \mathrm{kg})$ of the classic KOPr agonist U69,593 leads to a significant increase of DAT function after $2 \mathrm{~h}$, and this effect is inhibited by prior treatment with the KOPr antagonist nor-BNI (Thompson et al. 2000).

Chronic treatment with KOPr agonists decrease DAT density in the caudate putamen (Collins et al. 2001a; Collins et al. 2001b) but decreases DAT function in the NAc (Thompson et al. 2000). This indicates that long-term administration of KOPr agonists have the opposite effect to acute treatment with agonists given as a single dose. However, there have also been studies reporting no change in DAT function after KOPr agonist addition, with the decrease in DA in the tissue solely due to decreased DA release through interactions with D2 autoreceptors (Izenwasser et al. 1998). Therefore, while stimulation of the KOPr affects the DA system, the role of DAT in this process is not completely clear.

SERT function also plays an important role in drug addiction due to involvement in depression and withdrawal symptoms. Common techniques used to measure SERT function in cells, synaptosomes, and in vivo tissue systems include high-speed chronoamperometry (HSC), fast scan cyclic voltammetry (FSCV), and radioactive uptake studies. These studies have characterised Michaelis-Menten kinetics for 5-HT uptake (Kokoshka et al. 1998; Zhu et al. 2005), clarified the ion dependence of SERT 
function (Ramamoorthy and Blakely 1999), highlighted differences in 5-HT uptake in different brain regions (Daws et al. 2005), determined the binding orders of substrates to SERT (Ramamoorthy et al. 1993), and defined the inhibition constants of drugs and therapies for SERT function (Kokoshka et al. 1998; Zhu et al. 2005; John and Jones 2007a). While FSCV and HSC both provide detailed kinetic information both in vivo and in vitro, they are technically difficult and require expensive, specialised equipment. Radioactive uptake studies have provided information on transporter densities and kinetics, but the technique is unable to measure real-time uptake of 5-HT or initial uptake kinetics due to the extended time required for radioligand addition and washing steps.

RDEV is an established electrochemical technique for the measurement of real-time DA and norepinephrine uptake and release in both cells and tissues (Meiergerd and Schenk 1995; Burnette et al. 1996; Earles and Schenk 1998; Burgess et al. 1999; Chen et al. 1999a; Chen and Justice 2000; Chefer and Shippenberg 2002). The RDEV set-up consists of three electrodes: a rotating glassy carbon electrode, a reference $\mathrm{Ag} / \mathrm{AgCl}$ electrode, and an auxiliary platinum electrode. The rotating glassy carbon electrode is rotated at $2000 \mathrm{rpm}$ which keeps the sample in the chamber well mixed and also leads to the formation of a diffusion layer around the submerged electrode (Earles and Schenk 1998). As the DA, norepinephrine, or 5-HT in this layer are oxidised to their quinone counterparts, they release 1 or 2 electrons, generating a current. As these substrates are oxidised, new species from the surrounding liquid move into the diffusion layer and are also oxidised. Therefore, as DA, norepinephrine, or 5-HT are transported into cells or tissue in the chamber there is a decrease in free monoamine substrate, leading to a decrease in oxidation, and a decrease in current. As this method can take up to 1000 measurements per second, real-time uptake can be measured in both cells in suspension and tissue homogenates.

The theory of RDEV is described by the Levich equation:

$I_{L}=0.62 n F A C D^{2 / 3} v^{-1 / 6} \omega^{1 / 2}$

in which $I_{L}$ is the limiting current, $\mathrm{n}$ is the number of electrons transferred per mole, $F$ is Faraday's constant $(96,485$ coulombs/equivalent of electrons), $A$ is the area of the electrode in $\mathrm{cm}^{2}, D$ is the diffusion coefficient of the electroactive species in $\mathrm{cm}^{2} / \mathrm{sec}, C$ 
is the concentration of the electroactive species in the bulk solution in $\mathrm{mM}, v$ is the kinematic viscosity of the solution in $\mathrm{cm}^{2} / \mathrm{sec}, \omega$ is the angular velocity of rotation in $\mathrm{rad} / \mathrm{sec}$, where $\omega=2 \pi N$, and $N$ is the number of rotations per second (Earles et al. 1998). This equation defines the current that is measured at the solid electrode surface. RDEV is a simple and relatively cheap technique to set up and gives detailed information about Michaelis-Menten kinetics (Earles et al. 1998), drug effects (Earles and Schenk 1999a; Thompson et al. 2000), ion dependency of transporters (Chen et al. 1999a; Bjorklund et al. 2007), and binding information (Chen et al. 1999a; Earles and Schenk 1999a).

There are two different methods of RDEV which can be used to determine kinetic values of monoamine transporters. The first is known as the zero trans model where a single addition of monoamine neurotransmitter is added to a single piece of tissue. This is repeated over a range of neurotransmitter concentrations, with each concentration being added to a fresh piece of tissue. The other method is known as the low to infinite trans model in which the neurotransmitter is added to the tissue sequentially from the lowest concentration to the highest, allowing the nA to reach baseline between each addition. This method gives comparable results to single addition studies (Povlock and Schenk 1997) and was used here to decrease the number of animals needed in the study.

At the beginning of this study there were no published data using RDEV to measure SERT function. However, a recent publication has used RDEV to measure SERT function in mouse whole brain synaptosomes, comparing uptake between SERT knockout and wild type mice (Hagan et al. 2010). It was found that SERT kinetics could be easily measured using RDEV, with wild type mice having much higher uptake than heterozygous and homozygous SERT knockout mice. The uptake was inhibited by the SSRI paroxetine hydrochloride (Hagan et al. 2010). This study demonstrated that RDEV could be used to sensitively measure SERT kinetics within tissue samples, with the differences seen in uptake between the three genotypes of mice not always able to be measured using other, less sensitive techniques. Radioactive uptake studies of SERT function, for example, have been unable to distinguish between the heterozygous SERT knockout mice and normal mice (Bengel et al. 1998).

A recent paper by Volz et al., also established the use of frozen tissue for RDEV experiments, measuring the activity of DAT and vesicular monoamine transporter 2 
(VMAT2) from frozen rat striatum (Volz et al. 2009). In this paper the brain regions of interest were removed, weighed, cooled in ice-cold KREBS buffer for up to $45 \mathrm{~min}$ and then stored dry at $-80^{\circ} \mathrm{C}$ for up to 8 weeks. Uptake by DAT was decreased by $55-60 \%$ after 1 to 8 weeks of freezing (Volz et al. 2009). The ability to carry out RDEV on frozen rat tissue is valuable as it allows for greater consistency (since measurements of frozen tissue can be carried out at the same time) when carrying out chronic experiments involving injecting animals over several days. This means that all the brain samples can be processed on one day within a short time period and frozen for RDEV measurements later. This leads to tighter data as the time of death after the last drug treatment is less variable between the animals; therefore, any changes to the transporter caused by the drug will still be present. As our laboratory carries out many experiments requiring drug treatment of animals over a number of days, one of our aims was to investigate whether tissue frozen for one week still had measurable SERT function.

In this chapter, we characterise SERT function in more commonly used RDEV samples: a cultured cell model and homogenised striatal tissue of rat brain, and find that SERT function is ion and temperature dependent, increased by activation of p38 MAPK and decreased by the SSRI fluoxetine (Prozac). Rat striata frozen for 1 week still exhibited SERT function, making it possible to freeze brain samples of rats after experiments that required chronic drug treatment, thus, increasing the number of brain regions that could be analysed and improving the accuracy of the results. KOPr activation was also shown in our study to affect 5-HT levels in the brain. At least some serotonergic neurons in the medial rostral ventral medulla express KOPr (Kalyuzhyny and Wessendorf 1999), and it has been suggested there is a direct link between KOPr and SERT (Schenk et al. 2000).

Some studies using acute KOPr agonist treatment have reported increased 5-HT in the rat and human neocortex through increased 5-HT release (Berger et al. 2006), although others have shown no change of 5-HT release in the dorsal and median raphe nucleus or the NAc (Tao and Auerbach 2002; Carlezon et al. 2006), or an inhibition of 5-HT release after KOPr addition in mouse hippocampal synaptosome preparations (Grilli et al. 2009). The interaction between KOPr and 5-HT also appears to have a role in addiction, especially in relation to cocaine abuse. Cocaine-induced locomotion was not inhibited by chronic KOPr agonist treatment after depletion of 5-HT by parachloroamphetamine, suggesting that 5-HT has a role in this KOPr effect (Zakharova et al. 2008b). After this treatment there was also a brain region-specific decrease in 
SERT in the olfactory tubercle, claustrum, endopiriform and lateral septal nucleus (Zakharova et al. 2008b). Another study carried out in squirrel monkeys found that fluoxetine as well as the KOPr agonist spiradoline led to inhibition of cocaine-induced reinstatement, and this effect was reversed for both drugs by the $5-\mathrm{HT}_{1 \mathrm{~A}}$ agonist $8-\mathrm{OH}-$ DPAT, implying that the effect of the KOPr agonist involves a 5-HT pathway (RuediBettschen et al. 2010). Overall, these data suggest that 5-HT plays an important role in the link between cocaine and the KOPr.

As DA and 5-HT play major roles in addiction, compounds that alter their function may be useful as pharmacotherapies. Classic KOPr agonists have anti-addictive properties in animal models, but also lead to side effects such as dysphoria and depression, especially after long-term use (Negus et al. 1997). While several KOPr agonists have been trialled in cocaine-abusing humans, these agonists have had little effect on drug taking behaviour (Walsh et al. 2001a; Preston et al. 2004). This may be due to the relatively low dose used in most studies since the drug needs to be tolerated by the patients, or it could be that the KOPr agonists investigated did not have high enough affinities for the KOPr. Therefore, while KOPr agonism has potential to attenuate drug taking in humans, the classical KOPr agonists do not show promise as pharmacotherapies. The KOPr agonist salvinorin A possesses a novel structure and has similar anti-addictive properties to classical KOPr agonists in rats self-administering cocaine (Morani et al. 2009). The two novel agonists DS-1-240 and DS-3-216 have also shown anti-addictive effects in rats self-administering cocaine (A. Morani, unpublished data). There have been many structural analogues of salvinorin A synthesised, and screening of these compounds has identified a new set of highly active KOPr agonists. As these compounds are highly selective and have a novel structure, they may have potential for use in anti-addiction pharmacotherapies, and therefore the effects of these compounds on DAT and SERT need to be investigated. 


\subsection{Aim}

The KOPr modulates DA and 5-HT levels in the brain, and this is partially thought to be due to changes in DAT and SERT function. The aim of this chapter was to investigate the effects of KOPr agonists on the function of DAT in a tissue model from drug naïve rats. This chapter also aimed to establish measurement of SERT function by RDEV in a cell model and minced rat striatal tissue and the effect of KOPr on SERT modulation was investigated. 


\subsection{Methods}

\subsubsection{Standard curve}

For the RDEV, each day before starting an experiment a standard concentrationresponse curve was created in KREBS buffer using different concentrations of DA. A linear regression was then calculated from the increase in current (nA) at each DA concentration so that data collected from tissue samples could be transformed into $\mu \mathrm{M}$ based on the measured $n A$ values.

\subsubsection{Tissue sample preparation}

Male Sprague-Dawley rats (250-325 g), housed in the School of Psychology animal facility of VUW, maintained on a 12-hour light/dark cycle and given free access to food and water were euthanized according to VUW animal ethics guidelines. Ethics approval was obtained from the VUW animal ethics committee prior to the study. Rats were decapitated after $\mathrm{CO}_{2}$ euthanasia, and the brain was rapidly removed and placed in an acrylic stereotaxic brain matrixes block (Alto, AgnTho's AB, Sweden). A $2 \mathrm{~mm}$ coronal section from between the interaural co-ordinates +9 to $+11 \mathrm{~mm}$ was removed, and the striatum (one side) and NAc (both sides) were removed. A $2 \mathrm{~mm}$ coronal section from between the interaural coordinates +12 to $+14 \mathrm{~mm}$ was also removed, and the medial PFC was dissected from the section. Tissue was weighed and then minced with an ice-cold razor blade on an ice-cold glass petri dish for $30 \mathrm{~s}$ in $300 \mu \mathrm{L}$ of ice-cold KREBS buffer. Samples that were not used straight away could be kept on ice for up to $20 \mathrm{~min}$ before a measurable loss in transporter function was seen. Samples that needed to be kept for longer than this were incubated in KREBS buffer in a $37^{\circ} \mathrm{C}$ incubator and aerated with $95 \% \mathrm{O}_{2} / 5 \% \mathrm{CO}_{2}$ (Carbogen). The minced tissue was then put into a microcentrifuge tube and left to settle. Tissue pieces were washed 8 times in warmed KREBS buffer allowing for tissue to settle between each wash. The tissue suspension was then added to the RDEV chamber (kindly donated by J. B. Justice Jr.) in $294 \mu \mathrm{L}$ of $37^{\circ} \mathrm{C}$ KREBS buffer. The rotating glassy carbon electrode (Pine Instruments, special order AFMDO3GC, Pennsylvania) was lowered into the chamber and rotated at 2000 $\mathrm{rpm}$ with an MSR rotator (Pine Instruments). A potential of $+450 \mathrm{mV}$ relative to the $\mathrm{Ag} / \mathrm{AgCl}$ reference electrode was applied to the electrode by an eDAQ potentiostat EA161 (eDAQ, NSW, Australia). The resulting current was recorded by an eDAQ ecorder 210 (eDAQ, NSW, Australia) and saved using Chart software (eDAQ, NSW, Australia). 


\subsubsection{Experimental procedure}

Once the nA reading in the absence of substrate had reached a stable baseline, the KOPr agonist (500 nM, unless stated otherwise) or vehicle control (DMSO-matched) was added for the required time followed by DA. For the low to infinite trans model (Meiergerd et al. 1994a; Povlock and Schenk 1997), increasing concentrations of DA were added, and the nA output was allowed to reach baseline between each addition. For single additions, once the $\mathrm{nA}$ had reached baseline, the single addition $(2 \mu \mathrm{M})$ was made and the measurement of $\mathrm{nA}$ was continued for $30 \mathrm{~s}$. For medial PFC experiments, once baseline had been reached the NET inhibitor desipramine-HCl (1 $\mu \mathrm{M}$, Sigma) was added for $5 \mathrm{~min}$ prior to the DA addition. For the nor-BNI experiments, tissue was incubated with $1 \mu \mathrm{M}$ nor-BNI (Sigma) in carbogen-aerated, $37^{\circ} \mathrm{C}$ KREBS buffer for 30 min in an incubator at $37^{\circ} \mathrm{C}$. Every $2 \mathrm{~min}$, after the tissue pieces had settled, the KREBS buffer was removed and fresh KREBS buffer containing $1 \mu \mathrm{M}$ nor-BNI was added. This washed the tissue and prevented tissue degradation. For the controls in this experiment, tissue was treated the same but not in the presence of nor-BNI. This incubation technique allowed other brain regions to be investigated for other studies during the $30 \mathrm{~min}$ incubation to save on the number of rats needed for the study.

For 5-HT tissue experiments, procedures were carried out as stated above except that 1 $\mu \mathrm{M}$ of the DAT inhibitor GBR12909 was used to inhibit DAT function. For frozen tissue samples, the protocol was followed as previously described (Volz et al. 2009). Briefly, rat striata were removed, weighed, and then frozen at $-80^{\circ} \mathrm{C}$ for 1 week. Before use, striata were thawed at $22^{\circ} \mathrm{C}$ for $20 \mathrm{~min}$, and then minced and washed as above. For experiments with fluoxetine $(10 \mu \mathrm{M}$, Tocris, UK) and anisomycin (1 $\mu \mathrm{M}$, Sigma), compounds were added $5 \mathrm{~min}$ before the first addition of 5-HT. For $\mathrm{Na}^{+}$and $\mathrm{Cl}^{-}$free assays, and temperature dependent assays, a single addition of 5-HT to a final concentration of $1.5 \mu \mathrm{M}$ was made after baseline had been reached.Between each measurement within an experiment, the rotating electrode was cleaned with milliQ water on a Buechler Microcloth (Pine Instruments) for $30 \mathrm{~s}$, rotating at $2000 \mathrm{rpm}$. After each set of experiments, the electrode was then cleaned by hand with a 0.05 micron aluminium slurry on a Buechler Microcloth (Pine Instruments) followed by milliQ water before initiating another set of experiments. This prevented inconsistency due to fouling of the electrode, a problem seen with 5-HT that is more serious than with DA as a substrate. 


\subsubsection{RDEV with GFP-hSERT transfected HEK-293 cells}

HEK-293 cells were seeded into $100 \mathrm{~mm}$ tissue culture dishes and transfected as described previously (Sections 2.1.2 and 2.1.3) except that they were transfected only with GFP-hSERT at a plasmid concentration of $1 \mu \mathrm{g} / \mathrm{mL}$ After $48 \mathrm{~h}$, when the cells were $80-100 \%$ confluent, RDEV experiments were conducted on a suspension of cells following detachment by trituration of the dish.

Cells were washed twice with $37^{\circ} \mathrm{C}$ saline $(138 \mathrm{mM} \mathrm{NaCl}, 4.1 \mathrm{mM} \mathrm{KCl}, 5.0 \mathrm{mM}$ $\mathrm{KH}_{2} \mathrm{PO}_{4}, 2 \%$ w/v D-glucose, $\mathrm{pH}$ 7.3) (Appendix 1) and then harvested by mechanical agitation with $37^{\circ} \mathrm{C}$ KREBS buffer saturated with $5 \% \mathrm{CO}_{2} / 95 \% \mathrm{O}_{2}$. Cell suspensions were then centrifuged at $300 \mathrm{~g}$ for $5 \mathrm{~min}$. The cells were resuspended in $1.2 \mathrm{~mL} 37^{\circ} \mathrm{C}$ KREBS buffer saturated with $5 \% \mathrm{CO}_{2} / 95 \% \mathrm{O}_{2}$, and stored in an incubator at $37^{\circ} \mathrm{C}$ until needed, but for no longer then $30 \mathrm{~min}$. For temperature dependent experiments cells were treated the same way as above, but the KREBS buffer was $20^{\circ} \mathrm{C}$ rather than $37^{\circ} \mathrm{C}$. For $\mathrm{Na}^{+}$-free experiments, the $\mathrm{Na}^{+}$in the KREBS buffer was replaced with choline (130 $\mathrm{mM}$ choline, $1.3 \mathrm{mM} \mathrm{KCl}, 2.2 \mathrm{mM} \mathrm{CalCl}_{2}, 1.2 \mathrm{mM} \mathrm{MgSO}_{4} .6 \mathrm{H}_{2} \mathrm{O}, 1.2 \mathrm{mM} \mathrm{KH}_{2} \mathrm{PO}_{4}, 10$ mM HEPES, $10 \mathrm{mM}$ D-glucose) (Appendix 1), and for $\mathrm{Cl}^{-}$-free experiments, $\mathrm{Cl}^{-}$was replaced with acetate $\left(130 \mathrm{mM} \mathrm{NaCH}{ }_{3} \mathrm{CO}_{2}, 1.3 \mathrm{mM} \mathrm{KCH} \mathrm{CO}_{2}, 2.2 \mathrm{mM} \mathrm{Ca}\left(\mathrm{CH}_{3} \mathrm{CO}_{2}\right)_{2}\right.$,

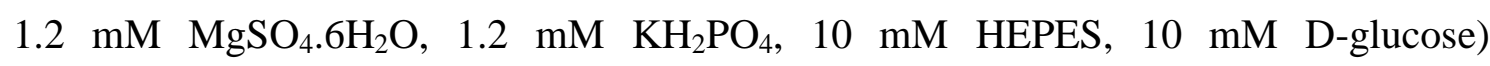
(Appendix 1).

For 5-HT cell uptake assays, $300 \mu \mathrm{L}$ of the cell suspension was added to the RDEV chamber, the rotator lowered and turned on, and the cells and incubated until a constant baseline was reached. Then a single addition of 5-HT was added to a final concentration ranging from 0.1 to $3 \mu \mathrm{M}$. Uptake was recorded for $30 \mathrm{~s}$. For $\mathrm{Na}^{+}$and $\mathrm{Cl}^{-}$free assays and temperature dependent assays, a single addition of 5-HT to a final concentration of $1.5 \mu \mathrm{M}$ was made after baseline had been reached. After each experiment the cells were placed into a separate tube and cell number was determined using a haemocytometer and $0.4 \%$ trypan blue. Transfection percentages were calculated by visualisation of eGFP-hSERT using a fluorescence microscope (AX70, Olympus, Mt Waverly, Australia). For experiments with anisomycin (Tocris, UK), a final concentration of $1 \mu \mathrm{M}$ was added 5 min before 5-HT addition. 


\section{$\underline{\text { 3.3.5 Data analysis }}$}

To measure the initial uptake of the tissue or cells, $10 \mathrm{~s}$ of uptake data was collected $1 \mathrm{~s}$ after addition of DA or 5-HT. These data were plotted in Microsoft Office Excel, and a linear regression was calculated. Any linear regression with an $\mathrm{R}^{2}$ value of less than 0.95 was not used in the study. The uptake of DA or 5-HT by the tissue sample over $1 \mathrm{~s}$ was calculated from the change in current and then transformed into $\mu$ mol using the standard concentration curve calculated at the beginning of the experiment. For tissue experiments, the uptake in $\mu \mathrm{mol}$ per $\mathrm{s}$ was then divided by the tissue weight in $\mathrm{g}$ to work out the uptake in $\mu \mathrm{mol}$ per s per g. This was then expressed in $\mathrm{pmol} / \mathrm{s} / \mathrm{g}$. For cell experiments, the uptake in $\mu \mathrm{mol} / \mathrm{s}$ was normalised to $10^{6}$ transfected cells and expressed as pmol $/ \mathrm{s} / 10^{6}$ cells. For the low to infinite trans model and cell kinetic experiments, initial uptake values were entered into GraphPad Prism, and a nonlinear regression (One-site binding hyperbole) was fitted to each repeat. The Vmax and Km values were then obtained for each repeat and then the average $\mathrm{Km}$ and Vmax values were calculated. 


\subsection{Results}

3.4.1 Effects of KOPr agonists on DAT function in brain tissue samples from drug naïve rats

Previous RDEV experiments have shown that acute injections of the KOPr agonist U69,593 into rats led to an increase in DAT function (Thompson et al. 2000). To investigate the effects of KOPr agonists on DA uptake in isolated brain regions involved in reward behaviour, the striatum, NAc and medial PFC from drug-naïve rats were incubated with the test compounds $(500 \mathrm{nM})$ for 1 or $4 \mathrm{~min}$ before addition of a single concentration of DA $(2 \mu \mathrm{M})$. Results showed that salvinorin A acted quickly, with a short duration. Increases in uptake of between $215 \%$ and $300 \%$ were observed by 1 min (Figure 3.1) in these tissue regions. The two novel compounds DS-1-240 and DS-3-216 both had effects similar to those of $\mathrm{U} 50,488 \mathrm{H}$, with increases in uptake occurring of $170 \%, 200 \%$, and 180-270\%, respectively, after $4 \mathrm{~min}$ in all brain regions (Figure 3.1). Uptake by DAT in the medial PFC is much lower than that in the striatum and NAc since the medial PFC has a much lower concentration of DAT (Sesack et al. 1998). By 4 min, the salvinorin A effect was lost, suggesting that it was degraded quickly by the tissue in the KREBS buffer.

The kinetics of uptake provides information on changes in affinity of a substrate for a transporter $(\mathrm{Km})$ and the maximum uptake velocity (Vmax). Changes in Vmax with no change in $\mathrm{Km}$ also indicate changes in cell surface expression of DAT, with a decrease in Vmax indicating decreased cell surface expression and an increase suggesting an increase in cell surface expression. Therefore, kinetic experiments were carried out to determine how the test compounds affected $\mathrm{Km}$ and Vmax values. The classic KOPr agonist U69,593 was also used in this experiment as a positive control for the kinetic data (Thompson et al. 2000). After a 1 min incubation with salvinorin A and a 4 min incubation with U69,593, U50,488H, DS-1-240, and DS-3-216, RDEV was carried out using the low to infinite trans model. These results showed that all of the agonists led to a significant increase in $V \max$ with no change in $\mathrm{Km}$ (Figure 3.2, Table 3.1). This suggests that an increase in cell surface expression occurs after incubation with these compounds. Because of the apparent short duration of salvinorin A and its unexpected kinetics, single addition studies were carried out using a lower concentration (100 nM) of salvinorin A to see if decreasing the amount added would decrease the possible degradation of the compound, which we proposed to have been fouling the electrode. This concentration is also the one used in the previous study that demonstrated 
increased uptake of DA after a 5 min incubation with salvinorin A (T. Shippenberg, unpublished data). Single additions of DA, added 1 or 4 min after incubation of NAc tissue with $100 \mathrm{nM}$ salvinorin A showed significant increases in DA uptake $\left({ }^{*} \mathrm{p}<0.05\right.$, One-way ANOVA followed by Bonferroni post test) (Figure 3.3). 

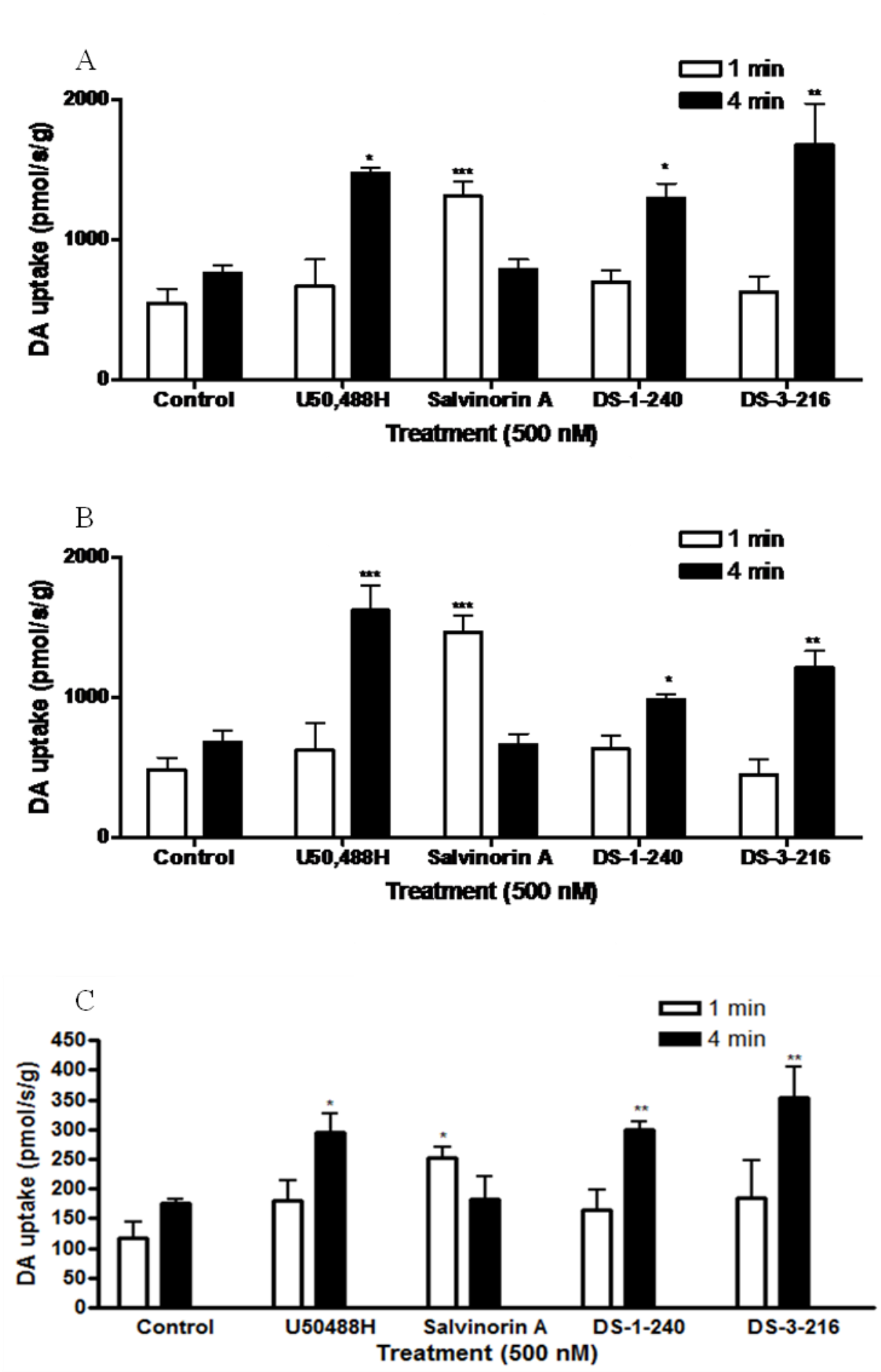

Figure 3.1: Effect of KOPr agonists on DA uptake in the striatum, nucleus accumbens, and medial prefrontal cortex. The striatum (A), NAc (B) and medial PFC (C) were pre-incubated with KOPr agonists for 1 or 4 min before DA $(2 \mu \mathrm{M})$ addition. Salvinorin A showed a fast onset of action, with an increase in uptake seen at 1 min that was lost by $4 \mathrm{~min}$, whereas U50,488H, DS-1-240, and DS-3-216 had their maximum effect after $4 \mathrm{~min}$. ( $\mathrm{n}=5-8$ tissue homogenates). One way ANOVA, followed by Bonferroni post test. $* p<0.05, * * p<0.01$, *** $p<0.0001$ compared to appropriate control. 


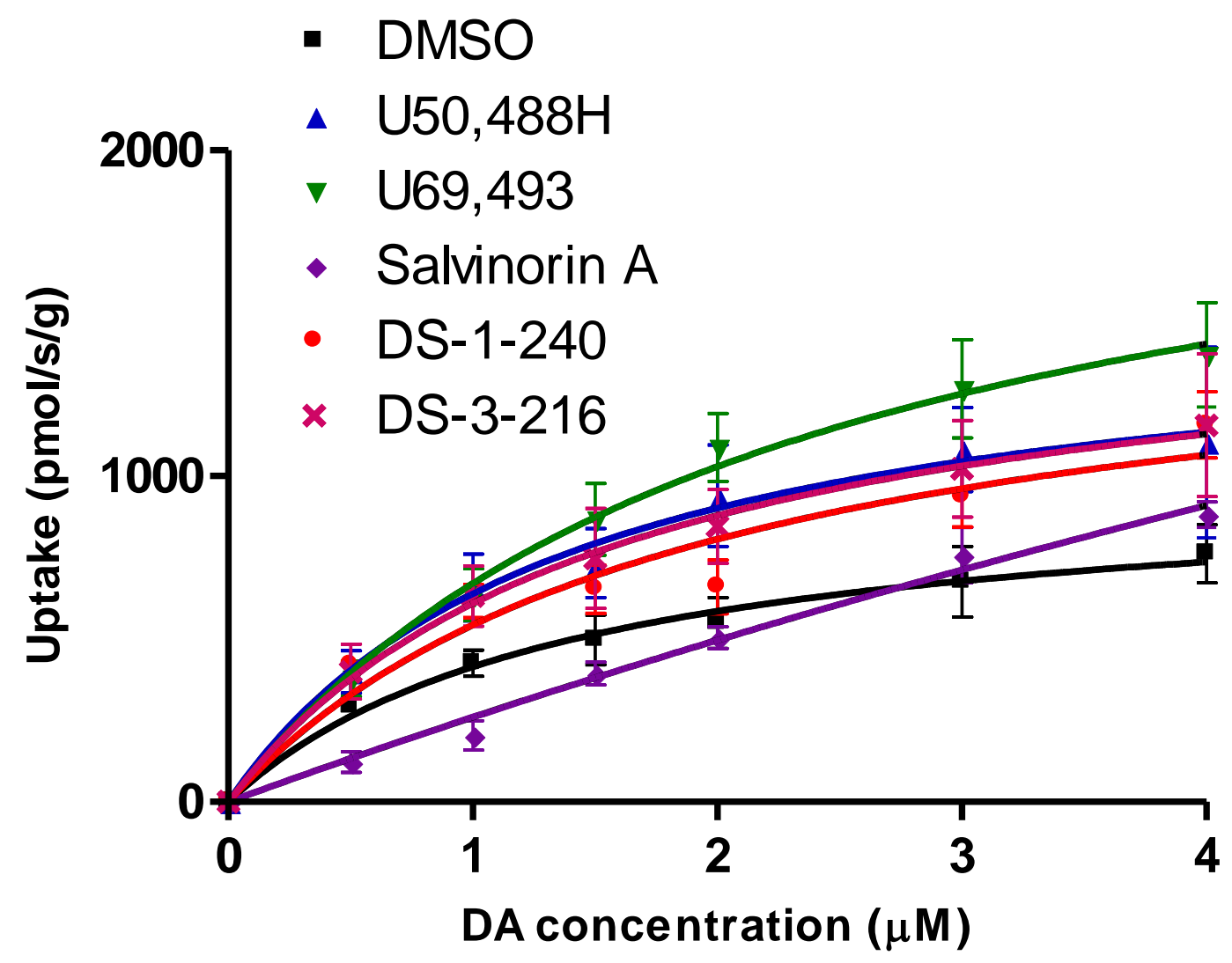

Figure 3.2: The effect of KOPr agonists on DAT kinetics in the nucleus accumbens. Nucleus accumbens tissue was pre-incubated with vehicle, the classic KOPr agonists U50,488H and U69,593, salvinorin A for $1 \mathrm{~min}$ or the novel KOPr agonists DS-1-240 and DS-3-216 for 4 min before sequential addition of DA. Data points shown are average \pm SEM $(n=6-8$ tissue homogenates $)$. 
Table 3.1: Uptake kinetics of DAT in the nucleus accumbens

\begin{tabular}{|l|l|l|}
\hline Treatment & Vmax $(\mathrm{pmol} / \mathrm{s} / \mathrm{g})$ & $\mathrm{Km}(\mu \mathrm{M})$ \\
\hline Control & $1026 \pm 181$ & $1.5 \pm 0.6$ \\
\hline U50,488H & $1979 \pm 335^{*}$ & $2.3 \pm 0.8$ \\
\hline U69,593 & $2424 \pm 255^{* *}$ & $2.7 \pm 0.6$ \\
\hline Salvinorin A & - & - \\
\hline DS-1-240 & $1509 \pm 115^{*}$ & $1.5 \pm 0.5$ \\
\hline DS-3-216 & $1592 \pm 176^{*}$ & $1.6 \pm 0.4$ \\
\hline
\end{tabular}

${ }^{*} \mathrm{p}<0.05,{ }^{* * *} \mathrm{p}<0.001$ compared to control of Vmax or Km. Student's t-test.

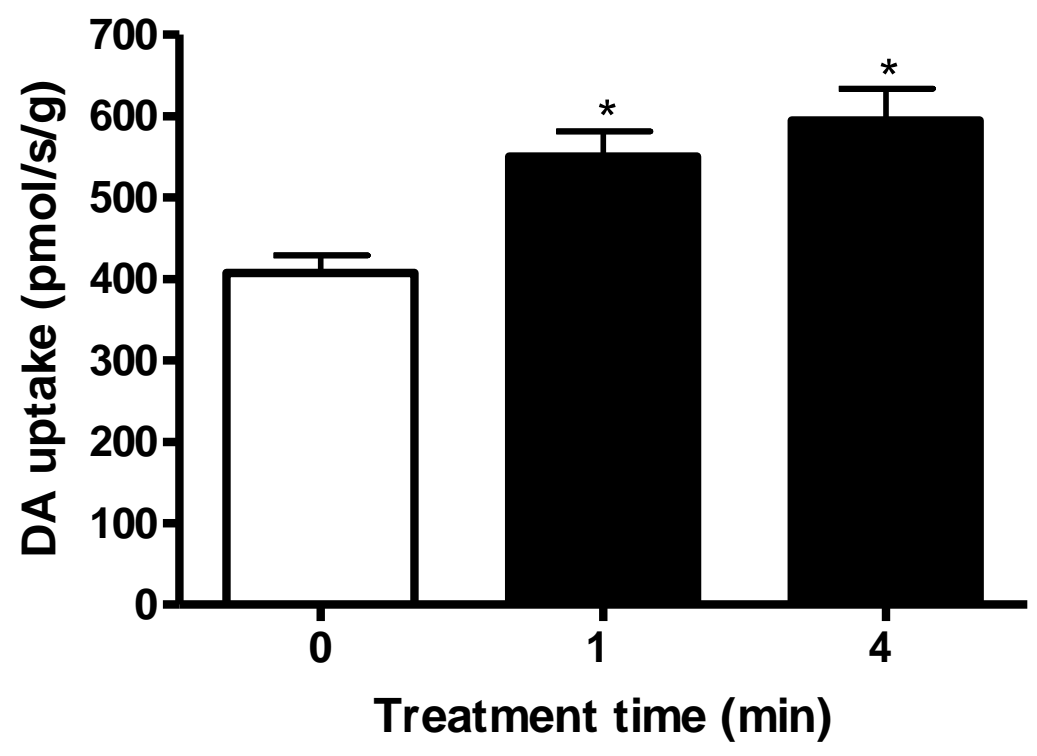

Figure 3.3: The effect of $100 \mathrm{nM}$ salvinorin A on uptake of $2 \mu \mathrm{MA}$ in the rat nucleus accumbens. Addition of $100 \mathrm{nM}$ salvinorin $A$ for 1 and 4 min caused an increase in DA uptake, One-way ANOVA followed by Bonferroni post test, ${ }^{*} \mathrm{p}<0.05$. ( $n=5-7$ tissue homogenates). 

$\underline{\text { nor-BNI }}$

To confirm that the increase in DAT function after KOPr agonist incubation was mediated through the KOPr, the KOPr antagonist nor-BNI was added 30 min prior to the incubation with the KOPr compounds. After rat striatal tissue had been minced, it was placed in a $37^{\circ} \mathrm{C}$ incubator in carbogen-bubbled (aerated) KREBS buffer containing vehicle (KREBS buffer) or $1 \mu \mathrm{M}$ nor-BNI. The KREBS buffer containing vehicle or nor-BNI was replaced with freshly aerated buffer every 2 min to wash the tissue and to prevent tissue death. The tissue was then transferred to the RDEV chamber, where, once a steady baseline had been reached, $500 \mathrm{nM} \mathrm{KOPr}$ agonist was added at $1 \mathrm{~min}$ (salvinorin A) or at 4 min (U50,488H, DS-1-240, DS-3-216). A single $2 \mu \mathrm{M}$ addition of DA was made, and uptake was measured. Incubation of tissue for $30 \mathrm{~min}$ prior to addition of KOPr agonist led to an inhibition of the agonists' effect. Figure 3.4a shows that all the compounds led to a significant increase in uptake compared to DMSO (Oneway ANOVA followed by Bonferroni post test, $\mathrm{p}<0.05-0.001)$. Prior incubation with nor-BNI led to a significant reversal of the effect of these compounds (One-way ANOVA followed by Bonferroni post test, $\mathrm{p}<0.05-0.001)$. Incubation of tissue with nor-BNI followed by treatment with vehicle led to no change in DA uptake (Figure $3.4 b)$. 

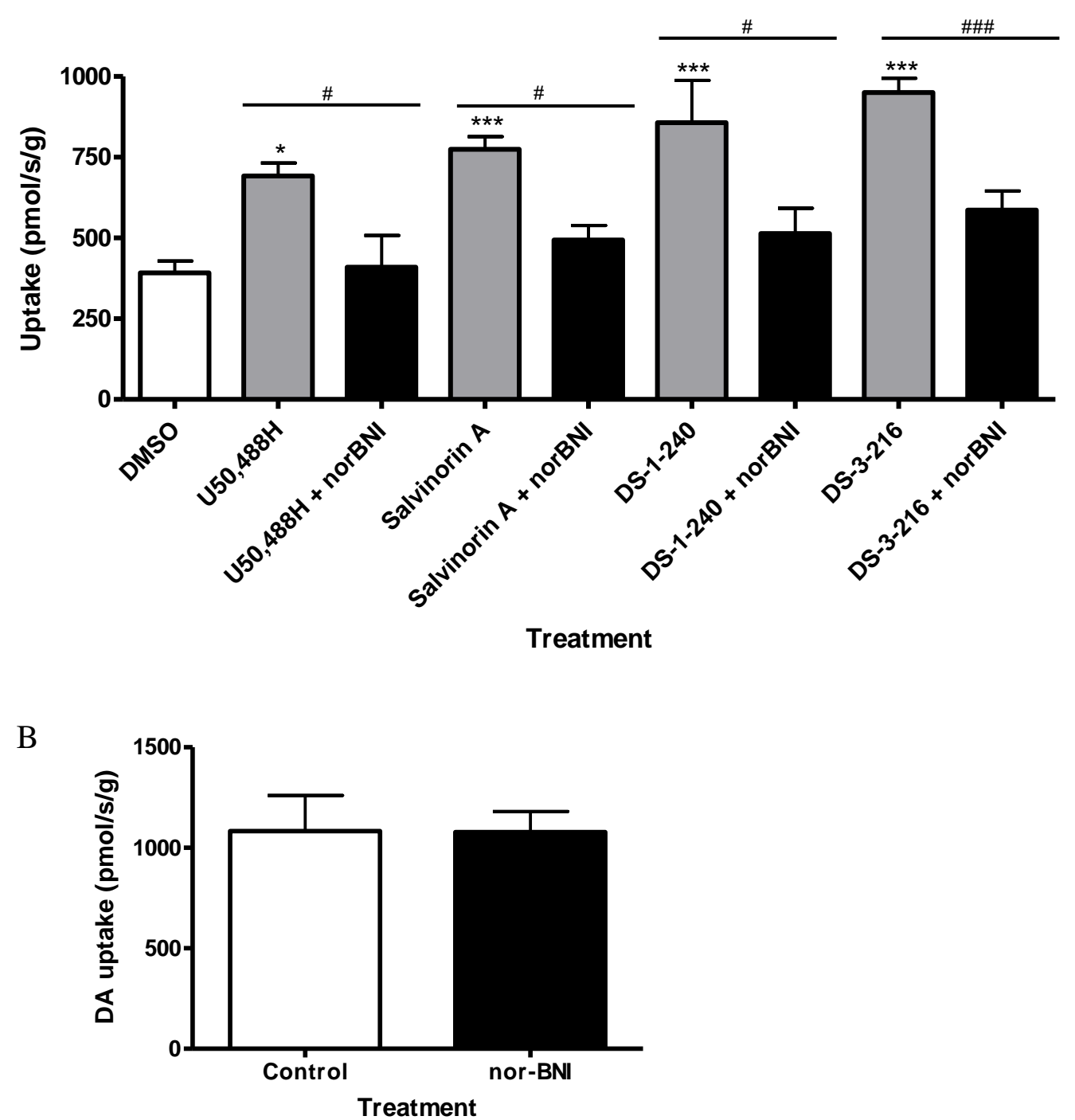

Figure 3.4: Nor-BNI inhibition of the effects of KOPr agonists on DAT function in the rat striatum. A) Incubation of striatal tissue with the $\mathrm{KOPr}$ agonists led to a significant increase in DA uptake, which was reversed by pre-incubation for $30 \mathrm{~min}$ with nor-BNI. B) Addition of nor-BNI on its own had no effect on DA uptake. Oneway ANOVA followed by Bonferroni post test, ${ }^{*} \mathrm{p}<0.05$, $* * * p<0.001$ compared to DMSO control, \#p<0.05, \#\#\#p<0.001 compared between agonist treated and nor-BNI pre-treated ( $n=3-6$ tissue homogenates). 
3.4.3 Establishment of RDEV for measurement of SERT in cells transfected with GFP$\underline{\mathrm{hSERT}}$ and in rat striatal tissue

\subsubsection{Transfected cell model}

Initial experiments were carried out using non-transfected HEK-293 cells that lack SERT to establish the non-specific decay or uptake of 5-HT in the system (Figure 3.5). The baseline decay occurred in a linear fashion, with the slope of the line equalling $6.355 \mathrm{pmol} / \mathrm{s} / 10^{6}$ cells $\left(\mathrm{r}^{2}=0.9891\right)$. The non-specific decay was subtracted from subsequent recordings obtained in GFP-hSERT transfected cells. The addition of 5-HT to HEK-293 cells transfected with GFP-hSERT led to uptake of 5-HT that followed Michaelis-Menten kinetics (Figure 3.5). An average Vmax of $92 \mathrm{pmol} / \mathrm{s} / 10^{6}$ cells and a $\mathrm{Km}$ of $1.91 \mu \mathrm{M}$ were obtained (Table 3.2).

\section{Effect of ions and temperature on 5-HT uptake}

As 5-HT uptake is $\mathrm{Na}^{+} / \mathrm{Cl}^{-}$dependent, with $1 \mathrm{Na}^{+}$and $1 \mathrm{Cl}^{-}$transported per 5-HT molecule, decreasing the concentrations of these ions should lead to decreased uptake of 5-HT (Ramamoorthy et al. 1992). Experiments were carried out with single additions of $5-\mathrm{HT}$ at $1.5 \mu \mathrm{M}$ as this is close to the $\mathrm{Km}$ value gained in the above kinetic experiments. When extracellular $\mathrm{Na}^{+}$was replaced with choline, uptake of 5-HT was significantly decreased compared to normal conditions (Figure 3.6), with only $24 \%$ of control uptake being obtained $(\mathrm{p}<0.001)$. When extracellular $\mathrm{Cl}^{-}$was replaced with acetate, a significant decrease in uptake was also observed at $29 \%$ of control uptake (Figure 3.6) $(\mathrm{p}<0.001)$. This demonstrates the importance of $\mathrm{Na}^{+}$and $\mathrm{Cl}^{-}$in 5-HT transport in this cell system. Uptake of 5-HT by SERT is temperature dependent, with maximal uptake occurring at $37^{\circ} \mathrm{C}$. When temperature was decreased to $20^{\circ} \mathrm{C}$, uptake was significantly decreased to only $16 \%$ of control uptake at $37^{\circ} \mathrm{C}(\mathrm{p}<0.001)$.

\section{Effect of the p38 MAPK activator anisomycin on 5-HT uptake}

p38 MAPK is a signalling pathway that activates SERT function, causing an increase in the affinity of SERT for 5-HT and no change in Vmax (Zhu et al. 2005). A $1 \mu \mathrm{M}$ concentration of the p38 MAPK activator anisomycin has previously been shown to increase SERT function in cell models after a 2 - 10 min incubation (Oz et al. 2010b). In the present study, after a 5 min incubation with anisomycin, there was a trend towards a decrease in $\mathrm{Km}(0.96 \mu \mathrm{M}, \mathrm{p}=0.1)$ with no change in Vmax (Figure 3.7, Table 3.2). 


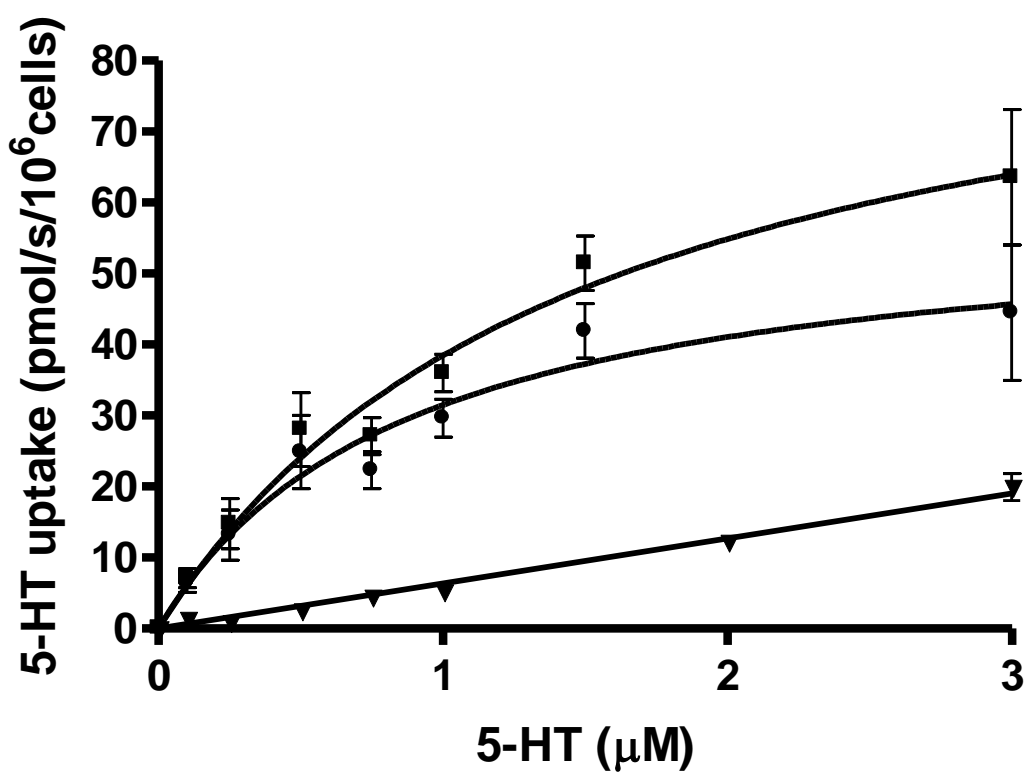

- Transfected

- Specific

von-transfected

Figure 3.5: Uptake of 5-HT in non-transfected and GFP-hSERT transfected HEK293 cells. Non-transfected and GFP-hSERT transfected HEK-293 cells were treated with single doses of 5-HT, and initial decay/uptake was measured by RDEV. Uptake from non-transfected cells (non-specific) decay was subtracted from transfected cell data (total) to give the specific initial uptake of 5-HT by GFP-hSERT. Data points represent the mean $\pm \operatorname{SEM}(\mathrm{n}=6$ preparations). 


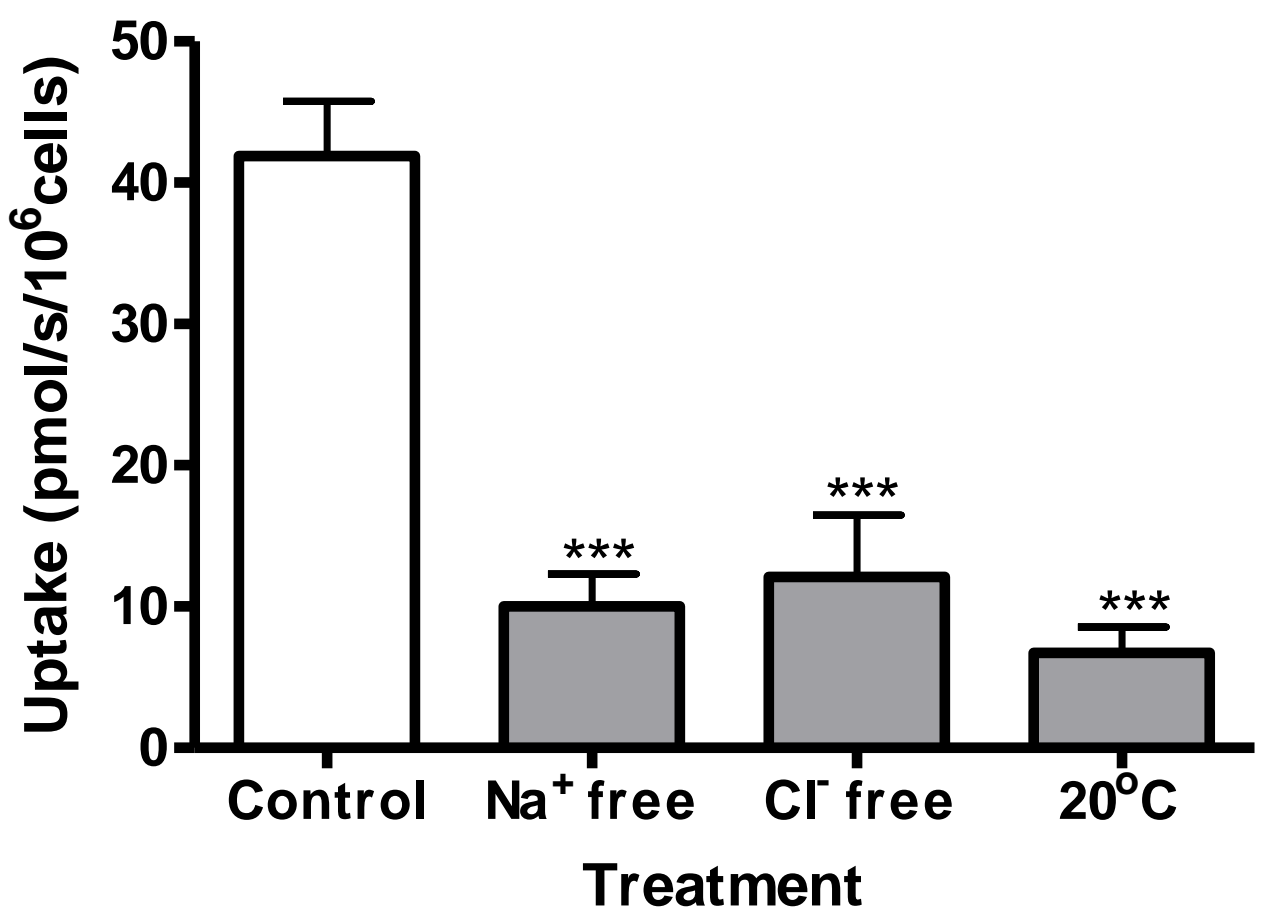

Figure 3.6: Ion and temperature dependence of 5-HT uptake in HEK-293 cells transfected with GFP-hSERT. Transfected cells were treated with a single $1.5 \mu \mathrm{M}$ dose of 5-HT in normal conditions, in $\mathrm{Na}^{+}$free conditions, in $\mathrm{Cl}^{-}$free conditions and at $20^{\circ} \mathrm{C}$. Uptake was measured by RDEV. Buffer lacking extracellular $\mathrm{Na}^{+}$or $\mathrm{Cl}^{-}$, or experiments carried out at $20^{\circ} \mathrm{C}$ had significantly decreased uptake compared to controls treated at $37^{\circ} \mathrm{C}$. Data points represent the mean \pm SEM ( $n=5-6$ preparations). Oneway ANOVA followed by Bonferroni post test, $* * * p<0.001$. 


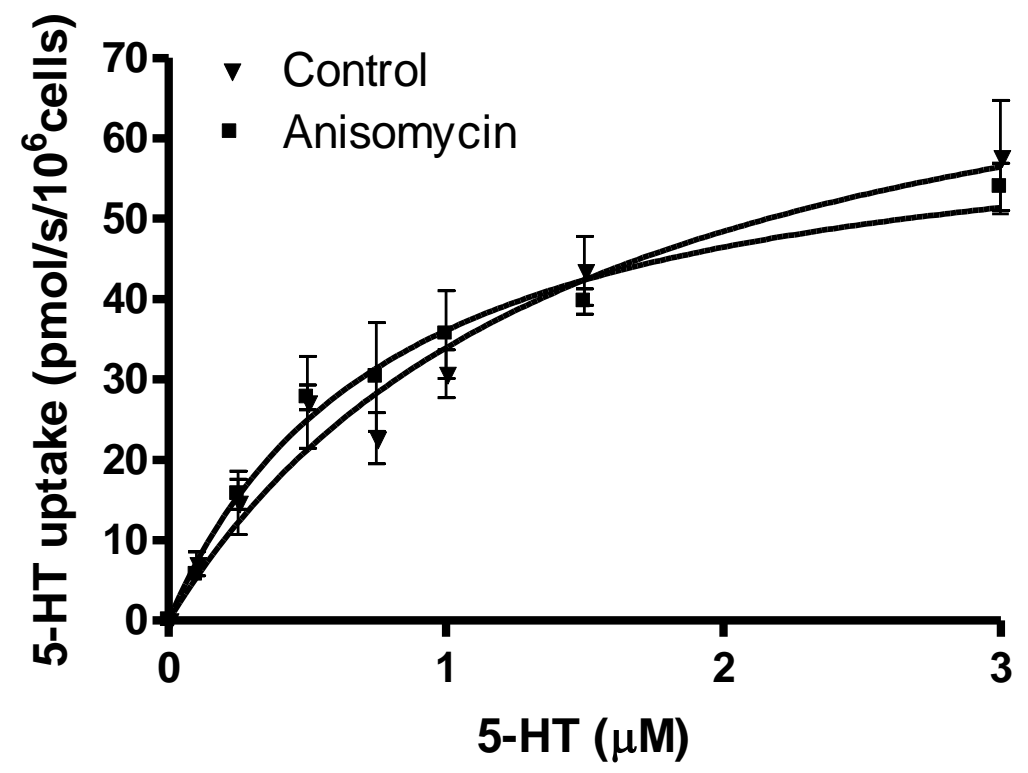

Figure 3.7: The effect of anisomycin on SERT function in HEK-293 cells transiently transfected with GFP-hSERT. Anisomycin $(1 \mu \mathrm{M})$ was added to cells for 5 min before uptake was measured by RDEV. Data points are mean \pm SEM ( $n=4-6$ preparations).

Table 3.2: Kinetic data from RDEV for control and anisomycin treated cells

\begin{tabular}{|l|l|l|}
\hline Treatment & Vmax $(\mathbf{p m o l} / \mathbf{s} / \mathbf{1 0}$ cells $)$ & $\mathbf{K m}(\boldsymbol{\mu M})$ \\
\hline Control & $92 \pm 6.8$ & $1.91 \pm 0.35$ \\
\hline Anisomycin & $86 \pm 2.3$ & $0.96 \pm 0.38(\mathrm{p}=0.1)$ \\
\hline
\end{tabular}

Values are the mean \pm SEM $(n=4-6$ preparations). Student t-test 


\subsubsection{Tissue model}

RDEV was carried out on rat striatal tissue blocked with the DAT antagonist GBR12909 (1 $\mu$ M, 5 min incubation (Zhou et al. 2005)) (Figure 3.8). Uptake studies carried out on rat striatal tissue in which DAT was not blocked led to a much higher level of 5-HT uptake compared to tissue incubated with GBR12909 (increases between 15 and $50 \%, \mathrm{Km}$ and $V \max$ values of $0.67 \mu \mathrm{M}$ and $642 \mathrm{pmol} / \mathrm{s} / \mathrm{g}$ wet weight, respectively, Table 3.3). DAT-inhibited uptake followed Michaelis-Menten kinetics, with average $\mathrm{Km}$ and $V \max$ values of $0.94 \mu \mathrm{M}$ and $466 \mathrm{pmol} / \mathrm{s} / \mathrm{g}$ wet weight, respectively (Table 3.3).

\section{Effect of ions and temperature on 5-HT uptake}

As mentioned above 5-HT uptake is $\mathrm{Na}^{+} / \mathrm{Cl}^{-}$dependent (Ramamoorthy et al. 1992). Experiments were carried out with single additions of $5-\mathrm{HT}$ at $1.5 \mu \mathrm{M}$ as this is close to the $\mathrm{Km}$ value gained in the above kinetic experiments. When extracellular $\mathrm{Na}^{+}$was replaced with choline, uptake of 5-HT was significantly decreased compared to normal conditions (Figure 3.9), with $71 \%$ of control uptake being obtained $(\mathrm{p}<0.001)$. When extracellular $\mathrm{Cl}^{-}$was replaced with acetate, a significant decrease in uptake was also observed at $73 \%$ of control uptake (Figure 3.9) ( $\mathrm{p}<0.001)$. This demonstrates the importance of $\mathrm{Na}^{+}$and $\mathrm{Cl}^{-}$in 5-HT transport in the rat striatum. Uptake of 5-HT by SERT is temperature dependent, with maximal uptake occurring at $37^{\circ} \mathrm{C}$. When temperature was decreased to $20^{\circ} \mathrm{C}$, uptake was significantly decreased to $55 \%$ of control uptake at $37^{\circ} \mathrm{C}(\mathrm{p}<0.001)$.

\section{Effect of the p38 MAPK activator anisomycin on 5-HT uptake}

As shown above p38MAPK leads to a trend towards decreased $\mathrm{Km}$ and no change in Vmax. Incubation of rat striatal tissue for $5 \mathrm{~min}$ with anisomycin led to a small increase in SERT function with a trend towards a decrease in $\mathrm{Km}(\mathrm{p}=0.07)$ and no change to Vmax (Figure 3.10, Table 3.3).

\section{Effect of the SERT inhibitor fluoxetine}

The SSRI, fluoxetine (Prozac), inhibits 5-HT uptake by SERT in the striatum (Rutter and Auerbach 1993). In the present study, the addition of fluoxetine (10 $\mu \mathrm{M})$ for $5 \mathrm{~min}$ before 5-HT addition led to a decrease of 5-HT uptake in the rat striatum (Figure 3.11) 
wtih a significant decrease in $\operatorname{Vmax}(\mathrm{p}<0.01)$ and $\mathrm{Km} \quad(\mathrm{p}<0.05)$ (Table 3.3), demonstrating down-regulation of SERT.

\section{Effect of one week of freezing on SERT activity}

A recent study showed that DA uptake can still occur in rat brain tissue that has been frozen at $-80^{\circ} \mathrm{C}$ for up to 8 weeks (Volz et al. 2009). Therefore, we wanted to validate the use of frozen tissues for uptake studies with SERT. After dissection, tissue was weighed and frozen at $-80{ }^{\circ} \mathrm{C}$ for 1 week, and then defrosted at $22^{\circ} \mathrm{C}$ for 20 min before mincing and using for RDEV experiments. 5-HT uptake still occurred in frozen tissue, but at a slower rate to that of fresh tissue (Figure 3.12). Vmax values were decreased to $76 \%$ of fresh tissue (466 to $354 \mathrm{pmol} / \mathrm{s} / \mathrm{g}$ ) but were not significantly different from nonfrozen tissue $(\mathrm{p}=0.1992)$. Km values were decreased to $64 \%$ of fresh tissue (0.94 to 0.61 ), but this decrease in Km was not significantly different from fresh tissue (Table $3.3)$. 


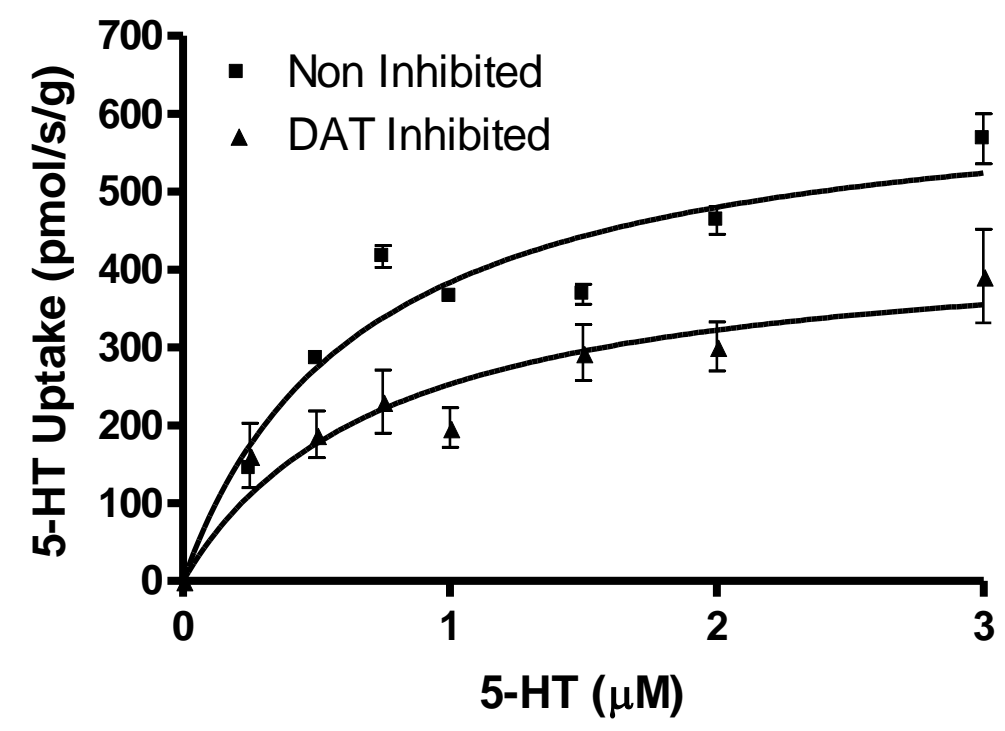

Figure 3.8: Inhibition of 5-HT uptake of DAT by GBR12909. DAT takes up 5-HT when 5-HT is present in high concentrations. Addition of the specific DAT blocker GBR12909 at $1 \mu \mathrm{M}$ decreased 5-HT uptake in the striatum, measured by RDEV,. This concentration has previously been used to inhibit 5-HT uptake by DAT ( $\mathrm{n}=3-6$ homogenates).

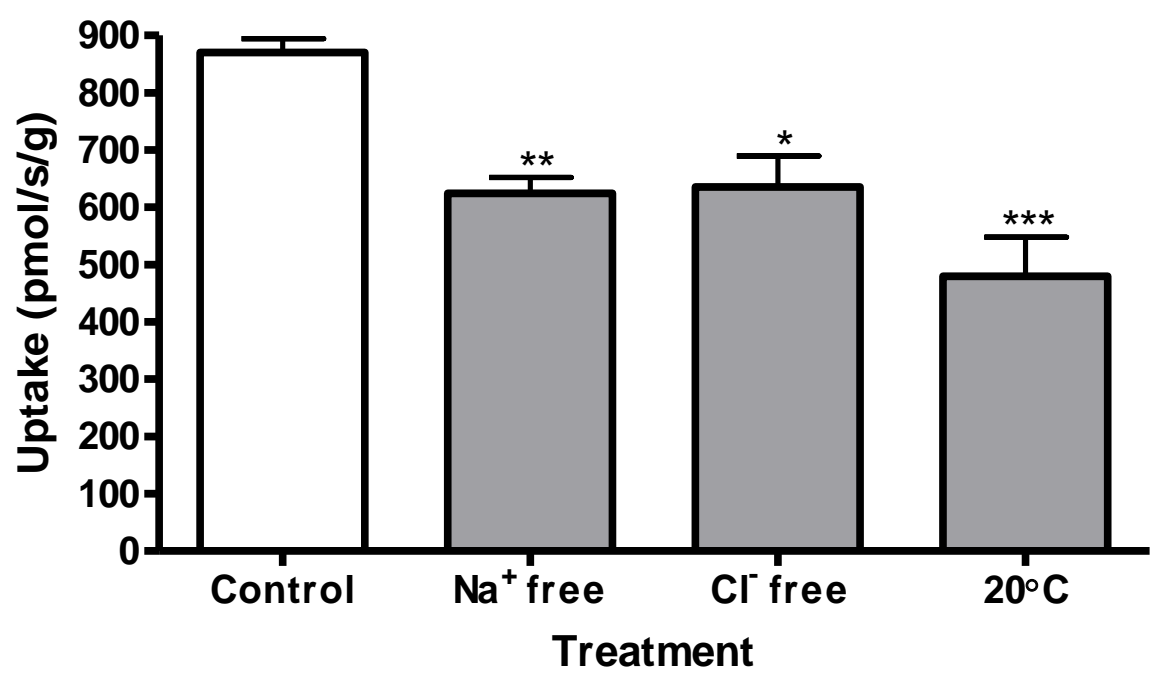

Figure 3.9: Ion and temperature dependence of 5-HT uptake in rat striatal tissue. After a 5 min incubation with GBR12909, rat striatal tissue was treated with a single 1.5 $\mu \mathrm{M}$ dose of 5-HT in normal conditions, in $\mathrm{Na}^{+}$free conditions, in $\mathrm{Cl}^{-}$free conditions and at room temperature. Uptake was measured by RDEV. Buffer lacking extracellular $\mathrm{Na}^{+}$or $\mathrm{Cl}^{-}$, or experiments carried out at $20^{\circ} \mathrm{C}$ had significantly decreased uptake compared to controls treated at $37^{\circ} \mathrm{C}$. Data points represent the mean \pm SEM ( $n=5-6$ preparations). One-way ANOVA followed by Bonferroni post test, ${ }^{*} \mathrm{p}<0.05,{ }^{*} \mathrm{p}<0.01$, $* * * \mathrm{p}<0.001$. 


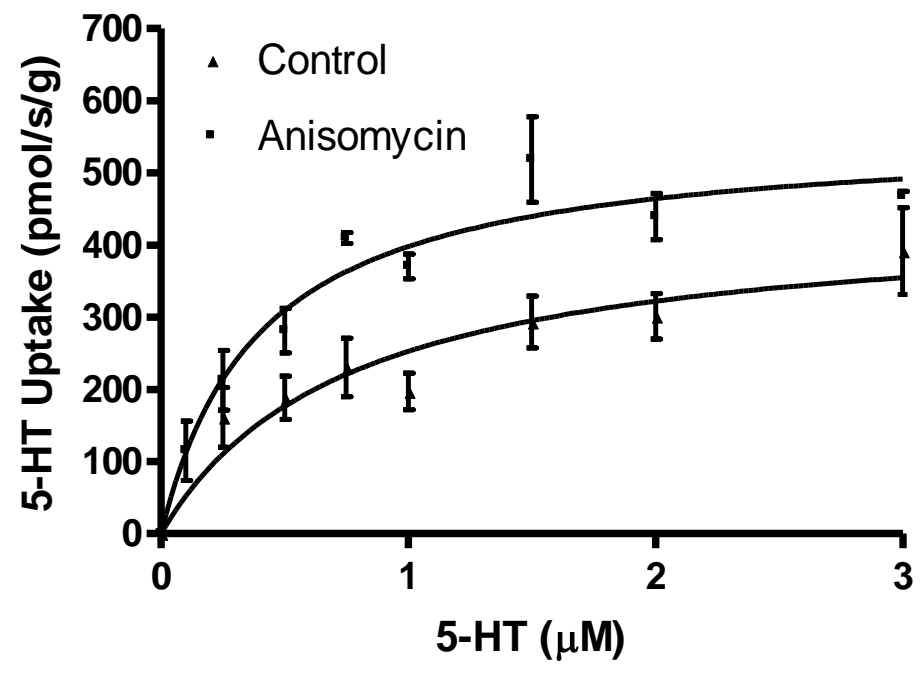

Figure 3.10: The effect of anisomycin on SERT function in rat striatal tissue. Anisomycin $(1 \mu \mathrm{M})$ was added to the tissue for $5 \mathrm{~min}$ before uptake was measured by $\mathrm{RDEV}$. Data points are mean \pm SEM ( $\mathrm{n}=4-6$ preparations).

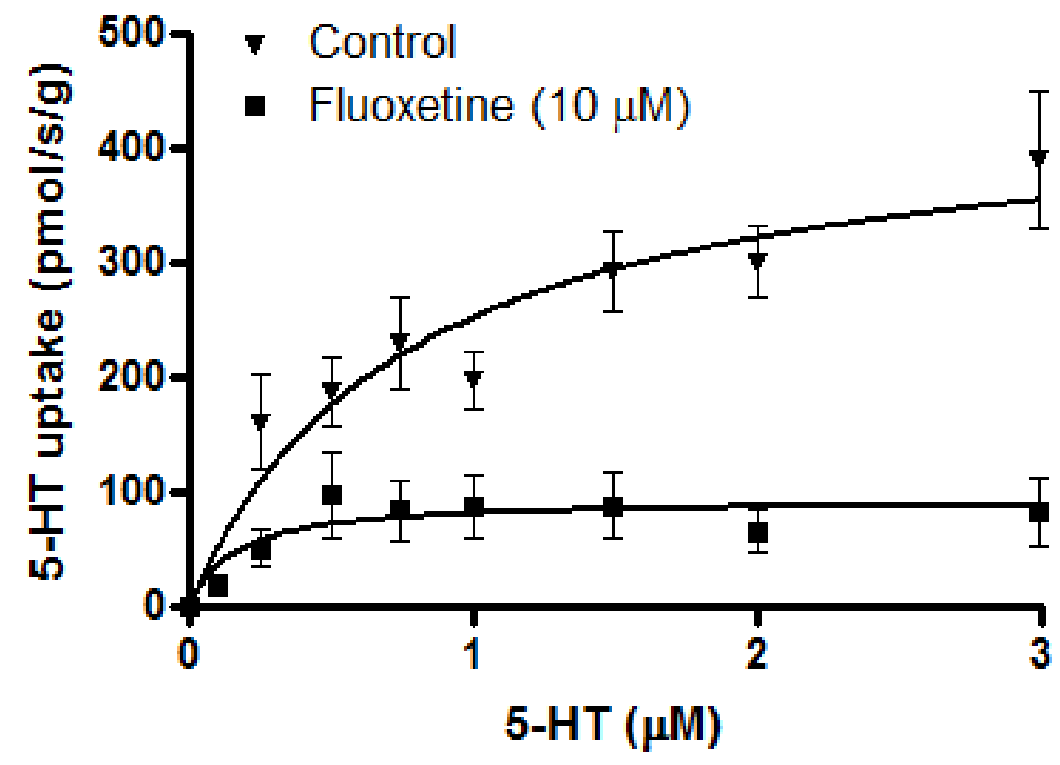

Figure 3.11: The effect of the SERT inhibitor fluoxetine on 5-HT uptake. Incubation with fluoxetine $(10 \mu \mathrm{M}) 5$ min after addition of GBR12909 $(1 \mu \mathrm{M})$ led to a decrease in 5-HT uptake in the rat striatum ( $\mathrm{n}=5-10$ homogenates $)$. 


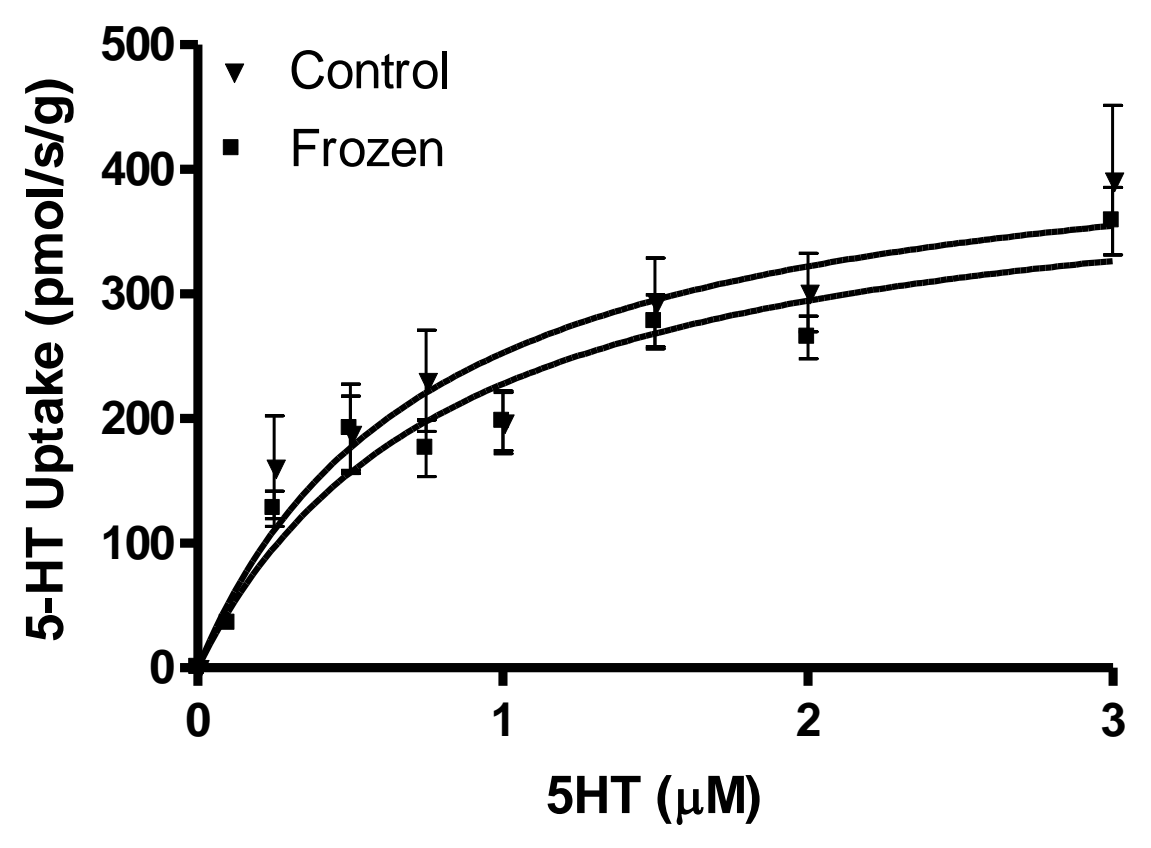

Figure 3.12: Uptake of 5-HT by SERT in the rat striatum and in frozen tissue samples. Rat striatal tissue was treated with GBR12909 5 min prior to measuring 5-HT uptake by RDEV. Rat striata were frozen for 1 week at $-80^{\circ} \mathrm{C}$ before defrosting at room temperature for $20 \mathrm{~min}$ and then using for 5-HT uptake studies ( $\mathrm{n}=4-6$ tissue homogenates).

Table 3.3: Kinetic data for tissue model

\begin{tabular}{|l|l|l|}
\hline Treatment & $\begin{array}{l}\text { Vmax } \\
(\mathbf{p m o l} / \mathbf{s} / \mathbf{g})\end{array}$ & $\mathbf{K m}(\boldsymbol{\mu M})$ \\
\hline Non-inhibited & $642 \pm 41$ & $0.67 \pm 0.05$ \\
\hline DAT-inhibited control & $466 \pm 61$ & $0.94 \pm 0.27$ \\
\hline Anisomycin & $550 \pm 53$ & $\begin{array}{l}0.40 \pm 0.11 \\
(\mathrm{p}=0.07)\end{array}$ \\
\hline Fluoxetine & $93.8 \pm 27^{* *}$ & $\begin{array}{l}0.1885 \quad \\
0.082^{*}\end{array}$ \\
\hline Frozen & $354 \pm 54$ & $0.61 \pm 0.25$ \\
\hline
\end{tabular}

Kinetic data were collected by RDEV, Student's t-test compared to the relevant control.

Data are the mean \pm SEM ( $n=4-6$ tissue homogenates). 


\subsubsection{The effect of KOPr agonists on SERT function in the striatum}

It has been suggested that the KOPr also regulates SERT function (Schenk et al. 2000). Activation of SERT by U69,593 leads to decreased 5-HT uptake in rat striatal synaptosomes (Ramamoorthy et al. 2002b). The effect of KOPr activation on SERT activity takes longer to occur compared to that of DAT (B. Kivell, personal communication); therefore, different time points were investigated to find when SERT function decreases after the addition of the classic KOPr agonist U50,488H. All tissues used for SERT experiments were first incubated with $1 \mu \mathrm{M}$ GBR12909 for $5 \mathrm{~min}$ to block DAT, which has been shown to take up 5-HT in addition to DA when 5-HT is present in high concentrations (Zhou et al. 2005). A significant $25 \%$ decrease in uptake of $2 \mu \mathrm{M}$ 5-HT occurred after a 15 min incubation with U50,488H (Figure 3.13), and this incubation time was therefore used for further studies. Salvinorin A and DS-1-240 also decreased 5-HT uptake by $25 \%$ in rat striatal tissue (Figure 3.14), demonstrating that KOPr also modulates SERT in the rat striatum. The effect of DS-3-216 on SERT function were unable to be measured due to the RDEV machine being broken at the time designated for the experiments. 


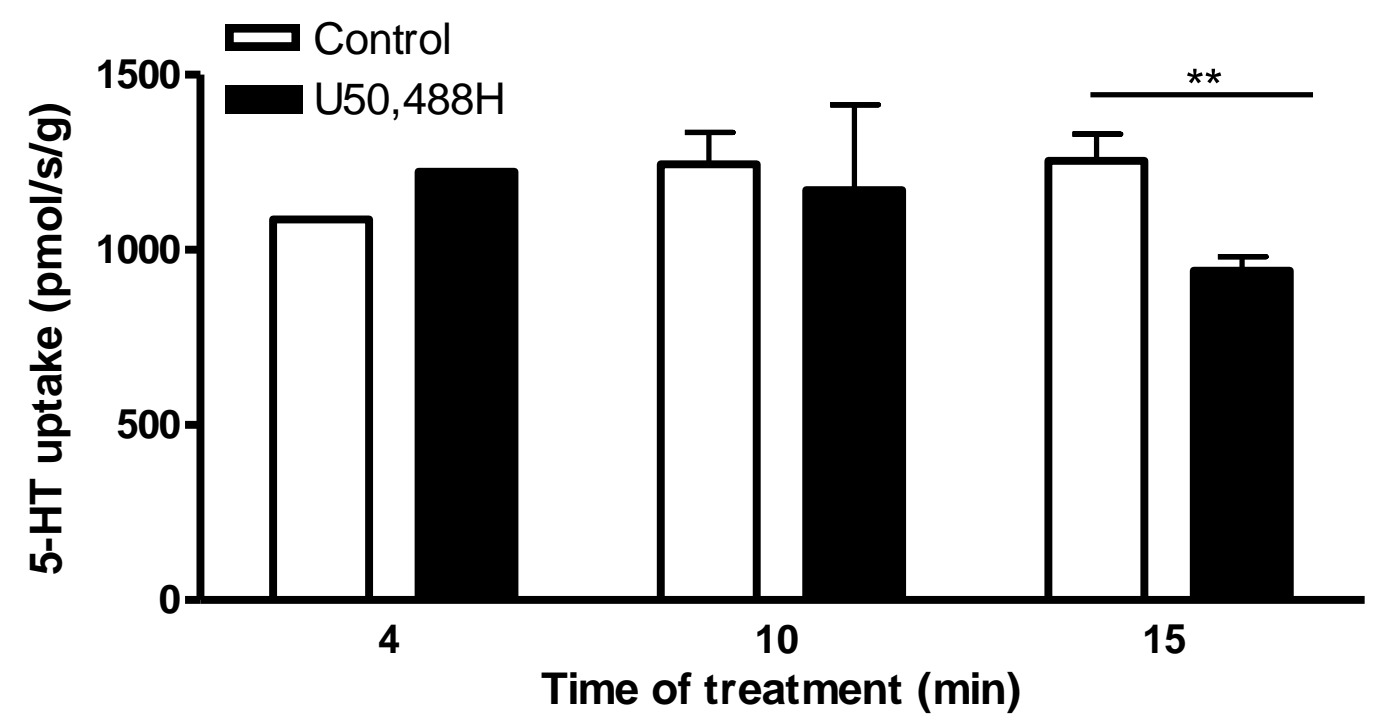

Figure 3.13: The effect of U50,488H on SERT function at 4, 10, and $15 \mathrm{~min}$. Rat striatal tissue was incubated with $500 \mathrm{nM}$ KOPr agonist U50,488H for 4, 10, and 15 min to find when it exerts its action. A significant decrease in 5-HT uptake was seen at 15 min (Student unpaired $\mathrm{t}$ test, $* * \mathrm{p}<0.01, \mathrm{n}=1-6$ tissue homogenates). This time point was used for all further studies with SERT and KOPr agonists.

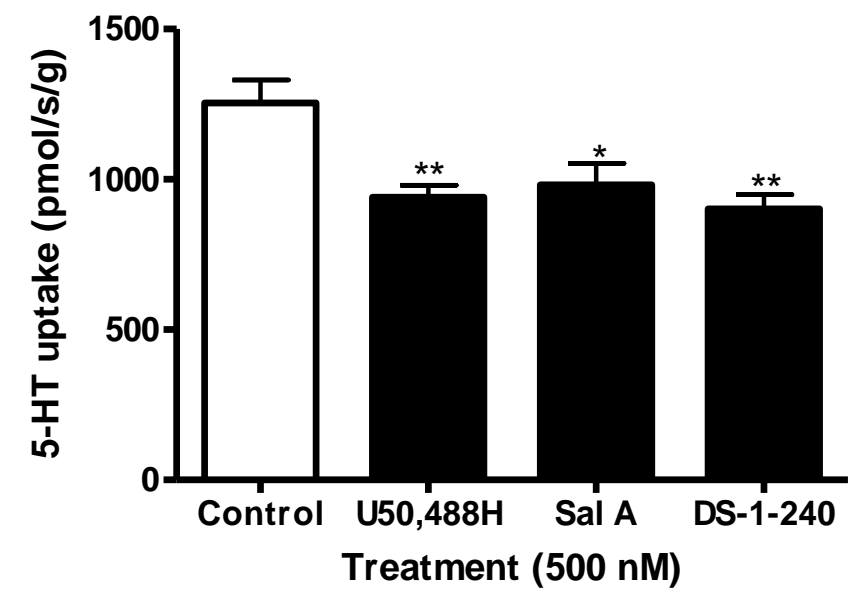

Figure 3.14: KOPr agonists decrease 5-HT uptake in the rat striatum. The striatum was treated for $15 \mathrm{~min}$ with $\mathrm{KOPr}$ agonist $(500 \mathrm{nM})$ and uptake of $2 \mu \mathrm{M} 5$-HT was measured using RDEV (One-way ANOVA, Bonferroni post test. $* \mathrm{p}<0.05$, $* * \mathrm{p}<0.01, \mathrm{n}=4-5$ tissue homogenates). 


\subsection{Discussion}

The dopamine transporter is one of the major proteins in the brain that is directly affected by drugs of abuse. All drugs of abuse either directly or indirectly lead to regulation of DAT, which causes short-term increases in DA in the synapse, where it can bind to DA receptors, and continue on reward signalling. As already mentioned, KOPr agonists attenuate the effects of acute cocaine challenge, and also prevent selfadministration of cocaine-primed cocaine seeking (Collins et al. 2001a; Collins et al. 2001b; Morani et al. 2009). This demonstrates the ability of KOPr to prevent drug taking after acute drug experiences and also after chronic drug taking. However, classic KOPr agonists have shown no significant effect on drug taking in limited human clinical trials (Walsh et al. 2001a; Preston et al. 2004). The interaction between the KOPr and DAT is a potential pharmacotherapeutic route if new compounds are found that affect drug taking in humans, and further investigation into the effects of the novel salvinorin-based compounds on this pathway may lead to identification of analogues that function differently from the classic agonists, giving rise to new therapeutics.

In this chapter the effects of the traditional KOPr agonists U50,488H, U69,593, and the novel compounds salvinorin A, DS-1-240, and DS-3-216 on DAT and SERT function in isolated brain regions was investigated. It was found that all of the KOPr agonists investigated increased DAT function in the striatum, NAc, and medial PFC. This increase in uptake occurred after $1 \mathrm{~min}$ for salvinorin A and after $4 \mathrm{~min}$ for $\mathrm{U} 50,488 \mathrm{H}$, DS-1-240, and DS-3-216. Kinetic studies showed that incubation with all compounds except salvinorin A led to an increase in Vmax but no change to $\mathrm{Km}$. Salvinorin A produced a linear substrate concentration vs. velocity graph, and therefore further studies were conducted using a lower concentration of this agonist as we postulated, as salvinorin A is known to be easily degraded, that it was being broken down in the RDEV chamber and fouling the electrode. Single addition uptake studies found that $100 \mathrm{nM}$ salvinorin A led to a significant increase in uptake after 1 and $4 \min (\mathrm{n}=4-6)$. The increased DAT function caused by the KOPr agonists was confirmed to be due to $\mathrm{KOPr}$ activation as prior incubation of the striatum with the KOPr antagonist nor-BNI led to inhibition of the effect of these compounds. The increased DAT function caused by the KOPr agonists was confirmed to be due to KOPr activation as prior incubation of the striatum with the KOPr antagonist nor-BNI led to inhibition of their effects. The KOPr agonists U50,488H, salvinorin A, and DS-1-240 also decreased SERT function in 
the striatum after a 15 min incubation, demonstrating the importance of KOPr for regulation of both DAT and SERT.

\subsubsection{The effect of acute DA additions in the striatum, NAc and medial PFC}

Pilot studies were conducted to investigate the time taken for the KOPr agonists to have an effect in minced rat striatal, NAc, and medial PFC tissue. These brain regions were chosen as they all play important roles in addiction and all three express both DAT and KOPr (Boja and Kuhar 1989; Meshul and McGinty 2000; Svingos et al. 2001; Mazei et al. 2002). The three brain regions investigated had varying levels of control DA uptake, with the striatum showing the largest uptake, followed by the NAc and then the medial PFC (Figure 3.1). This correlates with the amount of DAT that is expressed in each brain region, with the striatum having the highest DAT density, followed by the NAc and then the medial PFC (Sesack et al. 1998; Thompson et al. 2000). The medial PFC expresses a large amount of NET, which is thought to be involved with the majority of DA uptake in this brain region (Mazei et al. 2002); therefore, the NET inhibitor desipramine- $\mathrm{HCl}$ was used in all medial PFC samples to inhibit NET function.

Addition of all of the KOPr agonists tested in the striatum, NAc, and medial PFC led to an increase in DAT function, decreasing extracellular DA by 170 to $300 \%$ (Figure 3.1). As mentioned earlier, previous studies have shown that KOPr regulates DA levels in these brain regions (Chefer et al. 1999; Gray et al. 1999; Chefer et al. 2000; Thompson et al. 2000; Carlezon et al. 2006), with the majority of studies show a decrease in basal DA in these regions. A possible shortcoming of the present study is that the effect of KOPr agonists on DAT function was studied in rat brain regions that had been isolated and then treated with the KOPr agonist, rather than removing brain regions from animals that had been treated in vivo with the agonist. While the data gained match previous studies in which animals were pre-treated with KOPr agonists, there may be subtle differences. For example, KOPr neurons projecting from the ventral tegmental area inhibit DA release in the medial PFC, but have no affect on DA release in the NAc (Margolis et al. 2006). This difference highlights the complexity of regulation within the brain, which is not accounted for in the simple model used in our study. However, the model used in our study does demonstrate that KOPr does modulate DAT and suggests that whole brain circuitry is not required for this modulation to occur. This suggests that further studies can be conducted in cell models. 
The KOPr agonists investigated all caused similar increases in DAT function between brain regions, with salvinorin A and $\mathrm{U} 50,488 \mathrm{H}$ showing the most variability (increases of $240 \%$ to $300 \%$ and $170 \%$ to $280 \%$, respectively). For these compounds, the largest increase was observed in the NAc, with the striatum and medial PFC exhibiting similar increases in uptake. There have been many studies that show differences between regulation of DA in these brain regions, especially between the NAc and striatum (Roitman et al. 1999; Wu et al. 2001; Richards and Zahniser 2009). There is also evidence that regulation of DAT by KOPr is different between these brain regions (Chefer et al. 1999; Thompson et al. 2000). Overall, DS-1-240 and DS-3-216 had similar effects on uptake, with increases of between $170 \%$ and $210 \%$ observed. Salvinorin A had a slightly greater effect than the other compounds studied, and also had a much earlier onset of action, which may correlate to its fast onset of action in the brain (Hooker et al. 2008).

Overall, acute treatment with the KOPr agonists investigated in our study increased DAT function in isolated rat striatum, NAc, and medial PFC, causing decreased extracellular DA. These data suggest that increased DAT function contributes to the decreased DA measured in these brain regions after KOPr activation. It also demonstrates that the novel salvinorin-based KOPr agonists investigated in this study have similar effects to the classic agonist U50,488H. As these novel compounds also cause decreased extracellular DA levels, they may have potential for use in pharmacotherapies for addiction, but further investigation into their effects in animal models of addiction would need to be conducted.

\subsubsection{The kinetics of DAT after addition of KOPr agonists in rat NAc tissue}

The electrochemical technique RDEV can be utilised for kinetic studies, with Km and Vmax values giving information on how DAT is being modulated by KOPr activation. All of the agonists studied caused a significant increase in Vmax, which suggests increased cell surface expression of DAT. Whether this increase occurs after a long- or short-term incubation with the agonists we cannot be sure, as with the low to infinite trans model, the KOPr agonist is present in the RDEV chamber during the entire experiment, and this can often be as long as an hour. Interestingly, salvinorin A at 500 $\mathrm{nM}$ gave a linear substrate concentration vs. velocity graph, which is normally representative of an ion channel. It is possible that this concentration of salvinorin $\mathrm{A}$ is altering the transporter to cause it to act as a channel (J. B. Justice, personal 
communication). Another possibility is that prolonged incubation of salvinorin A within the chamber causes degradation of salvinorin A to its metabolite salvinorin B, which may foul the electrode.. Salvinorin A is an unstable compound that is rapidly degraded to its inactive metabolite salvinorin B by carboylesterase and serine esterase in rat plasma (Tsujikawa et al. 2009). To further investigate whether this effect was seen due to the concentration of salvinorin A used experiments were carried out at a lower concentration $(100 \mathrm{nM})$. We found that this concentration caused a significant increases in DAT function at both 1 and 4 min.

Overall these data help to give a broad understanding about how these compounds are modulating DAT function. The experiments show that the two classic agonists and the novel agonists have similar effects on DAT, with a possible increase in cell surface expression of DAT.

\subsubsection{The effect of KOPr agonists on DAT function is nor-BNI reversible in the rat} $\underline{\text { striatum }}$

Nor-BNI is a KOPr antagonist that has long-term effects in inhibiting KOPr agonism (Horan et al. 1992). To confirm that the increase in function that was found with the KOPr agonists was KOPr-mediated, rat striatal tissue was incubated with nor-BNI for 30 min prior to KOPr agonist addition. Addition of nor-BNI completely inhibited the effect of all the KOPr agonists studied. This demonstrates that all of the compounds investigated cause increases in DAT function in a KOPr specific method.

3.5.4 Establishing RDEV to measure SERT function in rat striatum and cells transfected with GFP-hSERT

When the present investigation was started, there were no submitted reports for the use of RDEV to measure SERT function; therefore, this chapter aimed to establish RDEV to measure SERT function in both cells transfected with hSERT and in rat brain tissue samples. A recent study by Hagan et al (2010), however, used RDEV to study SERT in mouse whole brain synaptosomes (Hagan et al. 2010), providing the first use of RDEV to measure SERT function. In our study, which was largely carried out before the Hagan et al. paper appeared, we wanted to establish the technique using samples that were commonly used for monoamine transporter functional assays. We found that 5HT oxidation was measureable in isolated cells and homogenised brain tissue by RDEV at $+450 \mathrm{mV}$. This differed from the method used by Hagan et al, who measured the 
current produced by 5 -HT oxidation at $+550 \mathrm{mV}$, but our values were still within the measureable range. Because of the lower voltage used, our 5-HT signal was 2.5 times lower than their reported value $(0.084 \pm 0.004 \mathrm{nA} / \mathrm{nM}$ compared to $0.21 \pm 0.01 \mathrm{nA} / \mathrm{nM})$. Some of this difference could be due to the nature of the preparation, i.e. synaptosomes versus cells and tissue. Apart from this, our protocols were very similar. Uptake of 5HT by SERT in cells transfected with GFP-hSERT was dependent on temperature and ion concentration and was possibly activated by the p38 MAPK activator anisomycin. These responses of SERT validate the RDEV method for measuring its function in transfected cells. Measurements were also taken in rat striatal tissue, and SERT was slightly down-regulated by fluoxetine and was also measurable in rat striatal tissue that had been frozen for one week.

HEK-293 cells transfected with monoamine transporters are often used for monoamine research as the cells are easily transfected and express the transporter at high levels. In this model, cells transfected with GFP-hSERT took up greater amounts of 5-HT than non-transfected cells (Figure 3.5) in a Michaelis-Menten manner. 'Non-specific' uptake in non-transfected cells was between $6-30 \%$ of the total uptake, with lower concentrations of 5-HT (below $500 \mathrm{nM}$ ) having much lower 'non-specific' uptake than higher concentrations (above $500 \mathrm{nM}$ ), as expected. Interestingly this was the opposite to what Hagan et al. found, in their study the lower concentrations of 5-HT used produced higher 'non-specific' uptake compared to higher concentrations. This difference may be due to the generally higher concentrations of 5-HT used in our experiments as Hagan et al. used $0.05 \mu \mathrm{M}$ to $1 \mu \mathrm{M}$ 5-HT (Hagan et al. 2010); whereas, in our experiments we used $0.1 \mu \mathrm{M}$ to $3 \mu \mathrm{M}$ 5-HT. 'Non-specific' uptake was subtracted from the total uptake in all cell experiments. The $\mathrm{Km}$ and Vmax values gained in our experiments $\left(1.91 \mu \mathrm{M}\right.$ and $92 \mathrm{pmol} / \mathrm{s} / 10^{6}$, respectively) fall within the range of previously reported values in transfected cell suspensions, which have a wide range of values for $\mathrm{Km}$ of from $0.30 \mu \mathrm{M}$ (Mao et al. 2000; Zhu et al. 2005; Tsuruda et al. 2010) to $21.8 \mu \mathrm{M}$ (Mochizuki et al. 2005). This large range of values is probably due to the different techniques used to collect the kinetic data, and the different SERT plasmid and cell types used to express SERT in. Most kinetic studies in cells are carried out using radioactive ligands; however, the Vmax data from radioligand uptake cannot be directly compared to the data gained in our study due to the different units used $\left(\mathrm{pmol} / \mathrm{s} / 10^{6} \mathrm{cells}\right.$ in our study vs. fmol/min $/ \mathrm{mg}$ protein in radioactive uptake experiments). 
We found that GFP-hSERT expressed in HEK-293 cells was sensitive to changes in $\mathrm{Na}^{+}$ and $\mathrm{Cl}^{-}$availability and temperature (Figure 3.6). SERT is a $\mathrm{Na}^{+} / \mathrm{Cl}^{-}$symporter and requires these ions in a 1:1:1 ratio with 5-HT for transport to occur (Ramamoorthy et al. 1993). Binding of the ions and 5-HT are crucial for uptake, though the order in which they bind to the transporter is not known (Chang and Lam 1998). The replacement of $\mathrm{Na}^{+}$with choline and $\mathrm{Cl}^{-}$with acetate led to overall decreased uptake to $24 \%$ and $29 \%$ of control uptake, respectively (Figure 3.6). This matches previously published data using hSERT (Ramamoorthy et al. 1992; Li et al. 2006; Oz et al. 2010b) in which uptake of radioactive 5-HT and $\mathrm{ASP}^{+}$was 10-30\% of control uptake for $\mathrm{Na}^{+}$-free conditions and 30-55\% of control uptake for $\mathrm{Cl}^{-}$-free conditions ( $\mathrm{Li}$ et al. 2006; Oz et al. 2010b). SERT function is also temperature dependent, with maximal uptake occurring at $37^{\circ} \mathrm{C}$. In the cell model in our study, decreasing the temperature to $20^{\circ} \mathrm{C}$ led to a significant decrease in 5-HT uptake, similar to that reported by others (Oz et al. 2010b). Overall, these data demonstrate that RDEV is sensitive enough to measure the dependency of SERT on ions and temperature to an equal degree as other more commonly used techniques.

There have been several studies identifying p38 MAPK as a regulator of SERT function (Samuvel et al. 2005; Zhu et al. 2005; Oz et al. 2010b). In these studies, activation of p38 MAPK causes a quick onset and short-lived increase in SERT activity (Zhu et al. 2005; Oz et al. 2010b). Inhibition of p38 MAPK has been shown to lead to a decrease in SERT cell surface expression (Samuvel et al. 2005). Anisomycin is a p38 MAPK activator that increases SERT function. The addition of anisomycin to the GFP-hSERT expressing cells in our study, however, caused a trend towards a decrease in $\mathrm{Km}$ and no change in Vmax, suggesting that there may be an increase in affinity of SERT for 5-HT but no change in cell surface expression. These data match that of Zhu et al., who also found that anisomycin $(1 \mu \mathrm{M})$ decreased the hSERT Km but caused no change in Vmax (Zhu et al. 2005). To confirm this further repeats could be conducted.

Brain tissue is often used in RDEV experiments since animals can be treated with compounds and the effects on brain transporter function later measured, or compounds can be applied directly to brain regions containing the transporter of interest, either by intracranial cannulae additions or isolated tissue exposures. Removal and analysis of a specific brain region, as carried out in our study, allows the effects of these compounds 
to be measured in brain regions that are directly involved in the pathways being investigated. Neuronal bodies are often found in different brain regions from their axons and synapses, where the transporters are found, and when severed, the cell closes up to form a complete unit, similar to a synaptosome. This allows uptake studies to be carried out on these isolated regions.

The striatum has a high density of SERT, but also contains DAT. DAT has been shown to take up 5-HT when it is present in high concentrations (Zhou et al. 2005). We carried out a control RDEV experiment to see if the addition of the specific DAT blocker GBR12909 $(1 \mu \mathrm{M})$ decreased uptake of 5-HT in the striatum, as this concentration has previously been shown by FSCV to inhibit 5-HT uptake by DAT in striatal brain slices (Zhou et al. 2005). GBR12909, however, has no effect on 5-HT uptake by its normal transporter SERT. In our RDEV experiments, addition of $1 \mu \mathrm{M}$ GBR12909 led to a significant decrease (15-50\%) in 5-HT uptake, and this concentration was therefore used for all further experiments to inhibit 5-HT uptake through DAT. This allows for specific conclusions to be made on SERT function in our experiments without the complication of DAT uptake of 5-HT. Uptake of 5-HT through SERT in rat striatal tissue followed Michaelis-Menten kinetics, with $\mathrm{Km}$ and Vmax values of $0.94 \mu \mathrm{M}$ and $466 \mathrm{pmol} / \mathrm{s} / \mathrm{g}$, respectively. Other studies using rat striatal tissue have reported a very wide range of $\mathrm{Km}$ values. $\mathrm{Km}$ and Vmax values are dependent on the technique used and the type of preparation, for example, chopped tissue or synaptosomes (Near et al. 1988). Km values are much larger in chopped tissue compared to synaptosomes due to the larger diffusion barrier of intact tissue pieces compared to a homogeneous mix of small diameter synaptosomes or cells. $\mathrm{Km}$ values in the literature range between $30 \mathrm{nM}$ for rat midbrain synaptosomes (Kokoshka et al. 1998; Samuvel et al. 2005) to $2.0 \mu \mathrm{M}$ for whole rat brain measured by FSCV (Daws et al. 2005). Our Km value falls within this wide range of values. As the previous RDEV study carried out on SERT by Hagan et al (2010) used mouse tissue, our data cannot be directly compared to their results either since kinetic values are probably species and brain region specific. However, their $\mathrm{Km}$ and Vmax values from synaptosome preparations also fell within the published range.

As seen in the cell studies, removing $\mathrm{Na}^{+}$or $\mathrm{Cl}^{-}$from the assay buffer and decreasing the temperature at which the experiment was carried out at led to a decrease in SERT function (Figure 3.9). While the effect seen with tissue was not as great as that seen in 
cells it was still significant. This difference may be due to the size difference between cells and tissue and although tissue was washed there would most likely still be ions present in the tissue. Treatment of tissue with anisomycin 5 min before 5-HT uptake experiments also led to a decrease in $\mathrm{Km}$ and no change in Vmax (Figure 3.10), agreeing with our cell data and previously published data (Zhu et al. 2005).

The SSRI anti-depressant fluoxetine is known to competitively act on SERT, preventing the uptake of 5-HT back into the neuron and thereby increasing synaptic cleft concentrations of 5-HT (Wang et al. 2006; John and Jones 2007b). In the present study the addition of fluoxetine significantly decreased 5-HT uptake and decreased both $\mathrm{Km}$ and Vmax, demonstrating inhibition of SERT.

A recent publication reported that isolated rat brain regions can be frozen at $-80^{\circ} \mathrm{C}$ and defrosted for use with RDEV (Volz et al. 2009). Being able to do this is useful for RDEV experiments as often many animals are treated with drugs over a long period of time. For the best consistency of the data, the animals need to be euthanized and RDEV carried out on all samples at about the same time after euthanasia so that the effect of the drugs on monoamine transporters are at similar levels when the animals are sacrificed. This means that changes in the transporter due to the drugs in the brain will be similar between animals. Freezing the tissue would also make it easier to carry out experiments when multiple brain regions are used. We therefore wanted to investigate whether we could carry out SERT functional assays on brain tissue frozen for one week. In our experiment brain tissue was frozen at $-80^{\circ} \mathrm{C}$ directly after removal and weighing rather than after removal and cooling, and similar results were observed compared to the previously published report (Volz et al. 2009) - some loss of function but not complete loss. There were also no problems in our study with frozen striatal tissue not settling sufficiently by gravity, which was an issue noted in the study by Volz et al. (2009). SERT function was still able to be measured after one week of freezing and with little loss of uptake capacity occurring compared to unfrozen controls in which uptake was measured immediately after removal from the animal. A previous study that investigated the effect of freezing of rat and human brain samples on the uptake of DA and 5-HT found that in rat forebrain there was a non-significant decrease in both $\mathrm{Km}$ and Vmax of radiolabelled 5-HT uptake with their freezing protocol, in which tissue was stored frozen at $-70^{\circ} \mathrm{C}$ for 1 to 3 weeks (Stenstrom et al. 1985). Overall the decrease in function in our study $(0-22 \%)$ was less than what was observed by Volz et 
al. with frozen tissue for DAT RDEV, in which decreases of 55-60\% were reported (Volz et al. 2006). It is uncertain whether this difference is due to the different properties of DAT and SERT, or if it is because our protocol does not including a prior 'cool down' period of the tissue before freezing. The lack of change in Km with freezing in our study suggests that the SERT transporters that remain functional have not been altered by freezing with regard to the binding and interaction of 5-HT with the transporter protein.

To conclude this section, RDEV was successfully established for measuring SERT function in cells transfected with GFP-hSERT and in rat striatal tissue samples. Measurements on DAT function by RDEV are relatively common; however, the use of RDEV for measuring SERT function was novel, with only one report of its use toward the end of the study, and that in synaptosome preparations, not cells and tissue homogenates. RDEV was able to measure changes in SERT function due to changes in ion concentration and temperature and generated consistent kinetic data to that seen following the addition of fluoxetine and anisomycin in cells and tissue samples. 5-HT uptake was also successfully measured in rat striatal samples frozen for one week. The utilisation of RDEV to measure SERT function in these samples will lead to an increase in information on SERT and the effect that drugs of abuse and potential therapies have on SERT function.

\subsubsection{The effect of KOPr agonists on SERT function in the rat striatum}

SERT is found in many different areas of the brain, and its main function is as a regulator of mood. SERT is also involved in many mental disorders and also addiction (Murphy et al. 2008). The serotonergic system is involved in cocaine abuse, with SERT inhibition leading to inhibition of the priming effect of cocaine in squirrel monkeys (Czoty et al. 2002), and inhibition of cocaine seeking during drug extinction and cueinduced reinstatement of cocaine in rats (Burmeister et al. 2003). SERT and KOPr have also been shown to interact, with 5-HT essential for some of the effects of KOPr. Treatment with KOPr agonists leads to side effects such as depression and dysphoria, which also implicate 5-HT in KOPr-mediated effects (Redila and Chavkin 2008). Therefore, as we have the ability to measure SERT function using RDEV, the effect of acute KOPr agonists on SERT function was investigated. Acute KOPr activation by U50,488H, salvinorin A, and DS-1-240 in our study led to a decrease in SERT function 
after a 15 min incubation. This increase in 5-HT in the synapse may be involved with the decrease in drug seeking seen in laboratory animals in cocaine reinstatement experiments.

5-HT has also been shown to regulate the DA pathway, with activation of 5-HT receptors regulating DA release (reviewed in Alex and Pehek 2007). Increased extracellular 5-HT leads to activation of 5-HT receptors and then downstream activation or inhibition of DA release. 5-HT receptors also play a role in the effects of drugs of abuse such as cocaine and morphine, with agonism of the 5-HT receptor subtype $2 \mathrm{C}$ in rat PFC leading to decreased cocaine-induced hyperactivity and also inhibited stimulus effects of cocaine (Filip and Cunningham 2003). Antagonism of these receptors leads to a potentiation of DA release in the NAc and striatum after cocaine treatment, but agonism of these receptors does not decrease the DA increase caused by cocaine, although it did inhibit morphine-induced DA release (Willins and Meltzer 1998; Navailles et al. 2004). These studies demonstrate that the 5-HT pathway is important for drug addiction, and pharmacotherapies that target 5-HT and DA pathways may be more effective in attenuating drug seeking then those that just target one. 


\subsection{Conclusion}

To conclude, this chapter demonstrated that acute KOPr agonism leads to increased DAT function in the striatum, NAc, and medial PFC and that this may be mediated by increased cell surface expression of DAT. Salvinorin A and its analogues showed similar effects to those of the classic KOPr agonists $\mathrm{U} 50,488 \mathrm{H}$ and U69,593. The increase in uptake observed for all of the compounds was nor-BNI reversible, confirming its effect to be mediated through the KOPr pathway. The effects of salvinorin A were non-significantly nor-BNI reversible, and with further repeats this may also reach significance. RDEV was also successfully used to measure SERT function in rat tissue and transfected cells. Addition of KOPr agonists led to decreased SERT function in the striatum which may play a role in the decreased cravings seen after $\mathrm{KOPr}$ agonist treatment in reinstatement experiments. Further investigation into how these compounds elicit their effects on DAT and SERT will give greater information on their function, will highlight potential differences between classic and novel KOPr agonists, and may lead to useful information for the development of successful pharmacotherapies for addiction. 


\section{Chapter 4: Effects of KOPr agonists on the function and expression of DAT in a cell system}

\subsection{Introduction}

Cell models are often used when investigating the effect of drugs on monoamine transporters as they allow for greater manipulation than is possible in the whole animal. Therefore, our aim was to investigate if the KOPr agonists U50,488H, salvinorin A, DS1-240, and DS-3-216 alter DAT function in a cell model as they do in a tissue model (Chapter 3), and if any increases seen are due to increased cell surface expression. Cell studies were conducted in mammalian HEK-293 cells co-transfected with myc-rKOPr and YFP-hDAT.

\subsubsection{4-(4-(dimethylamino)-styrl)- $N$-methylpyridinium $\left(\mathrm{ASP}^{+}\right)$uptake}

$\mathrm{ASP}^{+}$is a fluorescent analogue of the neurotoxic compound 1-methyl-4phenylpyridinium $\left(\mathrm{MPP}^{+}\right)$which is used to induce Parkinson's Disease-like phenotypes in laboratory animals (Schwartz et al. 2003). $\mathrm{ASP}^{+}$is a substrate for the monoamine transporters and fluoresces red when it comes into contact with lipids; therefore, it can be used to investigate binding and uptake through monoamine transporters in live cells in culture using confocal microscopy (Schwartz et al. 2003). This technique is unique as it allows differentiation between binding and uptake of $\mathrm{ASP}^{+}$. It is a very sensitive method as it takes real-time, space-resolved measurements in single cells. Once inside the cell $\mathrm{ASP}^{+}$binds irreversibly to the mitochondria. $\mathrm{ASP}^{+}$uptake has been utilised to measure DAT (Bolan et al. 2007; Zapata et al. 2007; Oz et al. 2010a), SERT (Oz et al. 2010b), and NET (Schwartz et al. 2003; Schwartz et al. 2005) activity in cells. Binding of $\mathrm{ASP}^{+}$to these transporters occurs rapidly, and uptake of $\mathrm{ASP}^{+}$occurs in a linear fashion over a period of at least $10 \mathrm{~min}$ (Figure 4.1), allowing for acute affects of drugs to be measured in a within-cell design. Chronic drug effects can also be measured in a between-cell manner by comparing cells that have been incubated with drug before $\mathrm{ASP}^{+}$uptake with cells incubated with vehicle. The technique was established using NET-transfected cells by Schwartz et al. (2003) who demonstrated that $\mathrm{ASP}^{+}$uptake by NET was $\mathrm{Na}^{+}, \mathrm{Cl}^{-}$, cocaine, and temperature dependent. These researchers also demonstrated $\mathrm{ASP}^{+}$uptake in cultured neurons and in cells transfected with SERT and NET (Schwartz et al. 2003). Further studies on NET investigated the binding and 
transport of $\mathrm{ASP}^{+}$and found that binding occurred rapidly, but only resulted in transport of $\mathrm{ASP}^{+}$in 1 out of 1000 events (Schwartz et al. 2005).

$\mathrm{ASP}^{+}$uptake studies have also been used to investigate how cellular receptors regulate DAT function. Using co-transfected cells, $\mathrm{ASP}^{+}$uptake was used to demonstrate modulation of DAT function by the dopamine receptor subtypes D2 (Bolan et al. 2007) and D3 (Zapata et al. 2007), and by the cannabinoid agonist anandamide (Oz et al. 2010a). This technique has also been adapted for high-throughput screening in both transfected cells and neuronal cultures (Mason et al. 2005). It has also been used to measure the effect of the human immunodeficiency virus-1 transactivating factor (Tat) on DAT function in transfected cells in order to examine changes in DAT function and expression in human immunodeficiency virus-associated neurological disease (Perry et al. 2010). More recently $\mathrm{ASP}^{+}$uptake by SERT has been characterised (Oz et al. 2010b). Uptake by SERT was sensitive to changes in $\mathrm{Na}^{+}, \mathrm{Cl}^{-}$, temperature, cocaine, fluoxetine and p38 MAPK.

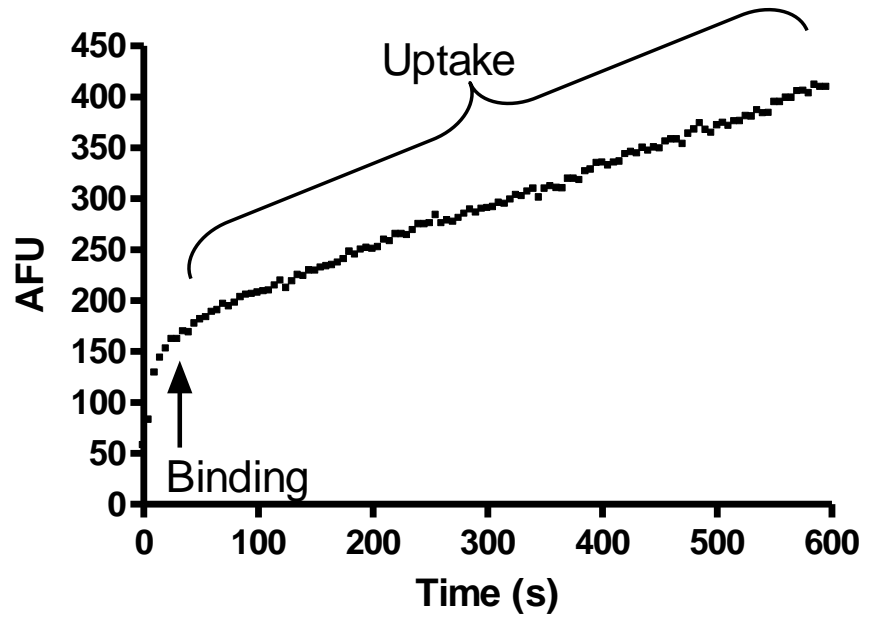

Figure 4.1: Binding and uptake of $\mathrm{ASP}^{+}$to YFP-hDAT HEK-293 cells. The initial binding phase is followed by a linear uptake phase for at least $10 \mathrm{~min}$ after $\mathrm{ASP}^{+}$ addition. 


\subsubsection{Total internal reflection fluorescence microscopy (TIRFM)}

TIRFM is a microscopy technique in which the laser beam is bent greater than a critical angle, allowing an evanescent field of only $100 \mathrm{nM}$ from the coverslip to penetrate the sample. The critical angle is given by:

$\theta_{c}=\sin ^{-1}\left(\mathrm{n}_{1} / \mathrm{n}_{3}\right)$

in which $\theta_{\mathrm{c}}$ is the critical angle, $\mathrm{n}_{1}$ the refractive index of the liquid, and $\mathrm{n}_{3}$ the refractive index of the solid (i.e. the glass bottom of the dish) (Axelrod 2001). The TIRF angle must be larger than the critical angle so that the laser beam is entirely internally reflected rather than refracted through the sample. TIRFM gives images that are free of background fluorescence compared to images gained by epi-fluorescence (Figure 4.2) and gives greater detail to the region of the sample that is adhered to the coverslip. TIRFM is useful for measuring changes that occur at the cell membrane of live cells containing fluorescent membrane proteins as the $100 \mathrm{nM}$ penetration depth allows visualisation of movement of fluorophores from the cytosol to the plasma membrane. The most studied transporter by TIRFM is glucose transporter 4 (GLUT4) which when stimulated by insulin acts to take up glucose into insulin-sensitive fat and muscle cells. From TIRFM experiments on adipocytes, it has been discovered that GLUT4 storage vesicles move within an intracellular tethering matrix to the cell surface ( $\mathrm{Li}$ et al. 2004a) These vesicles move along a microtubule network (Chen et al. 2008), and in the presence of insulin, they are tethered to the plasma membrane in clatherin-coated pits (Huang et al. 2007). Over time, these vesicles fuse with the plasma membrane in an actin-dependent (Lopez et al. 2009), v-SNARE-dependent manner (Zhao et al. 2009), leading to increased GLUT4 in the plasma membrane (Lizunov et al. 2005).

TIRFM has also been used to investigate the cell surface expression of DAT and SERT. DAT studies in $\mathrm{N} 2 \mathrm{~A}$ cells expressing YFP-hDAT have shown that DA and amphetamine cause an initial increase in DAT cell surface expression within $30 \mathrm{~s}$, followed by a decrease in expression levels. This effect was SNARE and PKC dependent and was inhibited by cocaine (Furman et al. 2009a). Also the effect of Tat on DAT cell surface expression was studied, with Tat causing an increase in DAT cell surface expression that was ryanodine receptor, calcium, and calpain mediated (Perry et al. 2010). A decrease in cell surface expression of SERT due to the effect of MDMA has also been demonstrated (Kivell et al. 2010). All these studies illustrate the ability of 
TIRFM to sensitively measure cell surface expression of fluorescently-tagged proteins, and to study the mechanisms by which changes in transport function occur.

\subsubsection{Cell surface biotinylation}

Cell surface biotinylation is another technique commonly used to measure changes in cell surface expression and is often partnered with TIRFM to confirm the results of TIRFM. It is generally not as sensitive as TIRFM as it measures changes in a pool of heterologous cells. This can be a particular issue with co-transfected samples since not all cells are transfected with the same number of plasmids, leading to variable results over different dishes. Also, as the sulfonate group of the EZ-link Sulfo-NHS-biotin can only bind to proteins found on the cell surface, proteins found within cell membrane invaginations are not always bound. This can lead to the internal fraction looking larger than it really is, as proteins in the invaginations are counted as internal rather than cell surface proteins (Furman et al. 2009a). However, this technique has been successful in demonstrating an increase in cell surface expression after DRD3 and DRD2 receptor activation (Bolan et al. 2007; Zapata et al. 2007), and after acute DA and amphetamine treatment (Furman et al. 2009a). Cell surface biotinylation has also successfully been used to demonstrate decreases in cell surface expression of DAT after incubation with the cannabinoid anandamide $(\mathrm{Oz}$ et al. 2010a), after 1-2 $\mathrm{h}$ incubations with amphetamine in NAc and striatal synaptosomes preparations (Richards and Zahniser 2009), after incubation with methamphetamine (Xie and Miller 2009) and after inhibition of MAPK pathways (Moron et al. 2003). This technique is useful as it can be used to investigate the effect of drugs of interest on brain tissue synaptosome preparations from animals that have been treated with the drug. This gives more relevant data to the in vivo situation compared to treating cells in vitro with the drugs of interest, especially if whole brain circuitry is required for some of the effects to be observed. 


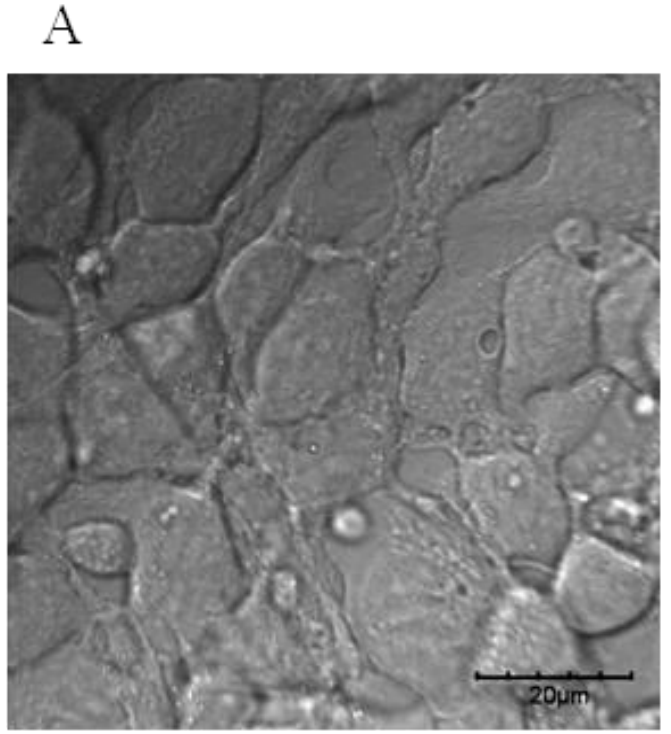

C

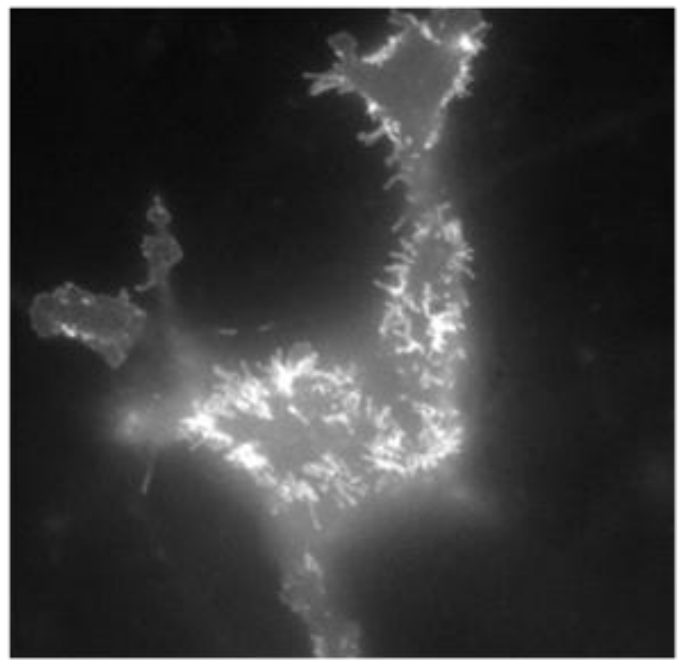

B

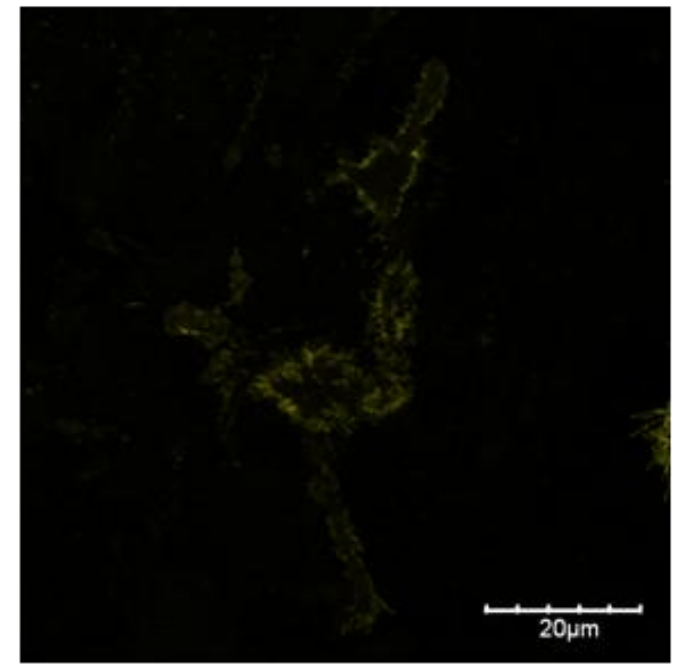

D

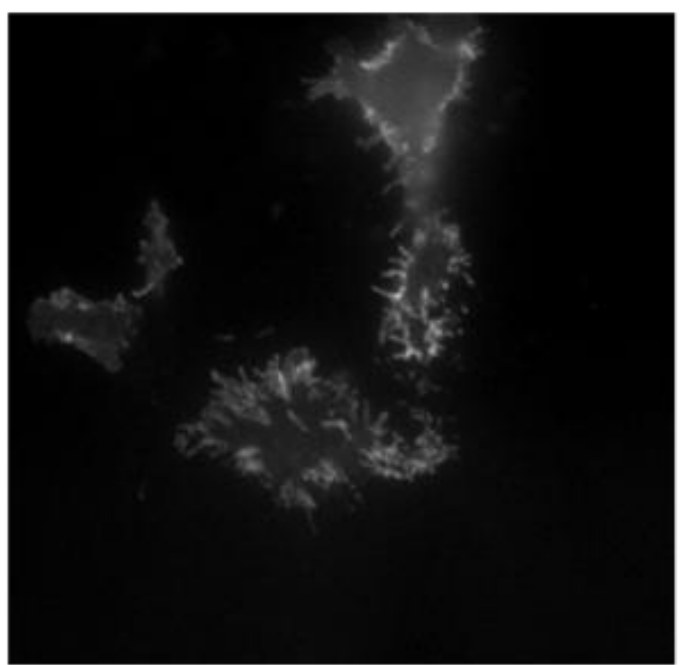

Figure 4.2: Comparison of DIC (A) confocal (B), epi-fluorscence (C) and TIRF (D) microscopy. Images were taken of the same cell using DIC (A), confocal (B), epifluorescence (C), and TIRFM (D). Notice the increased detail and clarity of the TIRFM image compared to the others.. 


\subsection{Aim}

The aim of this chapter, therefore, was to use confocal microscopy and cell surface biotinylation to investigate the cellular effects of other salvinorin A-based compounds such as DS-1-240 and DS-3-216 on the function and expression of YFP-hDAT in cells co-transfected myc-rKOPr in addition to YFP-hDAT. The effects of the KOPr antagonist nor-BNI and the $\mathrm{G}_{\mathrm{i} / \mathrm{o}}$ inhibitor PTX on transporter function was also explored. It is hypothesised that the novel KOPr agonists would cause concentrationdependent increases in $\mathrm{ASP}^{+}$uptake, which will be inhibited by nor-BNI and PTX, and that this increase in uptake will occur as a result of an increase in YFP-hDAT cell surface expression in these cells. Results gained from this chapter will confirm data gained from previous studies on salvinorin A and give new information on the effects of the novel KOPr agonists DS-1-240 and DS-3-216. 


\subsection{Methods}

\section{$\underline{4.3 .1 \text { Confocal microscopy }}$}

HEK-293, COS7, and N2A cells were cultured and transfected as described in section 2.1.2 and 2.1.3. Two days after transfection, cells were used for the confocal microscopy $\mathrm{ASP}^{+}$uptake experiments as described in section 2.5.

\subsubsection{Cell surface biotinylation}

HEK-293 cell surface biotinylation was carried out $48 \mathrm{~h}$ after transfection, as described in section 2.3 .

\subsubsection{Total internal reflection fluorescence}

TIRFM was carried out on transfected HEK-293 cells $48 \mathrm{~h}$ after transfection. as described in section 2.6.

\section{$\underline{\text { 4.3.4 Cell staining to visualise myc-rKOPr }}$}

Transfected HEK-293 cells were stained $48 \mathrm{~h}$ after transfection. The cells were first fixed in ice-cold methanol:acetic acid (1:1) for $7 \mathrm{~min}$ at room temperature and then washed $3 \mathrm{x}$ in PBS for 5 min each wash. Cells were blocked with PBS containing $0.25 \%$ Tween-20 and 1\% BSA for $30 \mathrm{~min}$ at room temperature. After blocking, the anti-myc antibody (1:10 dilution, Abcam) was added to PBS containing 1\% BSA and the cells were incubated overnight at $4^{\circ} \mathrm{C}$. The antibody solution was then removed and the cells washed $3 \mathrm{x}$ in PBS containing $0.25 \%$ tween-20 for 5 min each wash, and then incubated with anti-mouse Cy5 secondary antibody (1:100 dilution, Amersham) for $1 \mathrm{~h}$ at room temperature in the dark. Cells were kept in the dark at $4{ }^{\circ} \mathrm{C}$ until they were imaged by confocal microscopy. YFP-hDAT was imaged with the $473 \mathrm{~nm}$ laser (YFP filter: $485 \mathrm{~nm}$ excitation; $545 \mathrm{~nm}$ emission), and Cy5 tagged myc-rKOPr was imaged using the $635 \mathrm{~nm}$ laser (Cy5 filter: $655 \mathrm{~nm}$ excitation; $695 \mathrm{~nm}$ emission). 


\subsection{Results}

\subsubsection{Initial considerations}

4.3.1.1 Co-expression of YFP-hDAT and myc-rKOPr in HEK-293, N2A, and COS7 cells HEK-293, N2A, and COS7 cells co-expressing YFP-hDAT and myc-rKOPr were fixed and stained to visualise both YFP-hDAT and myc-rKOPr. It was found that in all three cell lines, myc-rKOPr and YFP-hDAT were co-expressed. Expression levels in COS7 cells, however, were much lower than those in HEK-293 and N2A cells even after optimisation of transfection protocols (Appendix 2).

\subsubsection{2 $\mathrm{ASP}^{+}$uptake method validation}

$\mathrm{ASP}^{+}$uptake and binding studies were conducted in HEK-293 cells co-transfected with myc-rKOPr and YFP-hDAT. Initial experiments confirmed that increased expression of YFP-hDAT led to increased $\mathrm{ASP}^{+}$accumulation at $200 \mathrm{~s}$ (Figure 4.4). Figure 4.5 shows $\mathrm{ASP}^{+}$accumulation in cells over a period of $300 \mathrm{~s}$ and demonstrates that uptake in YFPhDAT and myc-rKOPr transfected cells is much higher than that in non-transfected cells. Other preliminary studies were conducted to find the time when the compounds had the largest effect on $\mathrm{ASP}^{+}$uptake. It was found that salvinorin A caused a maximum uptake 60-120 s after $\mathrm{ASP}^{+}$addition, with increases in uptake seen within the first $60 \mathrm{~s}$ of addition. The classic KOPr agonist U50,488H had a longer onset of action, and had its maximal effect at 100-160s. Compounds DS-1-240 and DS3-214 showed an even longer lag period, and their maximum effects were seen after 200-260 s (Table 4.1, Appendix 2). These time points for the maximal uptake rates for each compound were then used for all experiments conducted. During these initial experiments the level of YFP-hDAT expression that gave functional changes in DAT after KOPr agonist addition was also verified as a ratio of at least two myc-rKOPr to one YFP-hDAT for functional changes to be observed (B. Kivell, personal communication). It was found that cells expressing YFP-hDAT that gave an AFU value of between 250 and 1000 showed increased $\mathrm{ASP}^{+}$uptake after KOPr agonist addition. Cells with an AFU for YFP-hDAT below 100 were classed as non-transfected as they did not show linear $\mathrm{ASP}^{+}$uptake curves. 

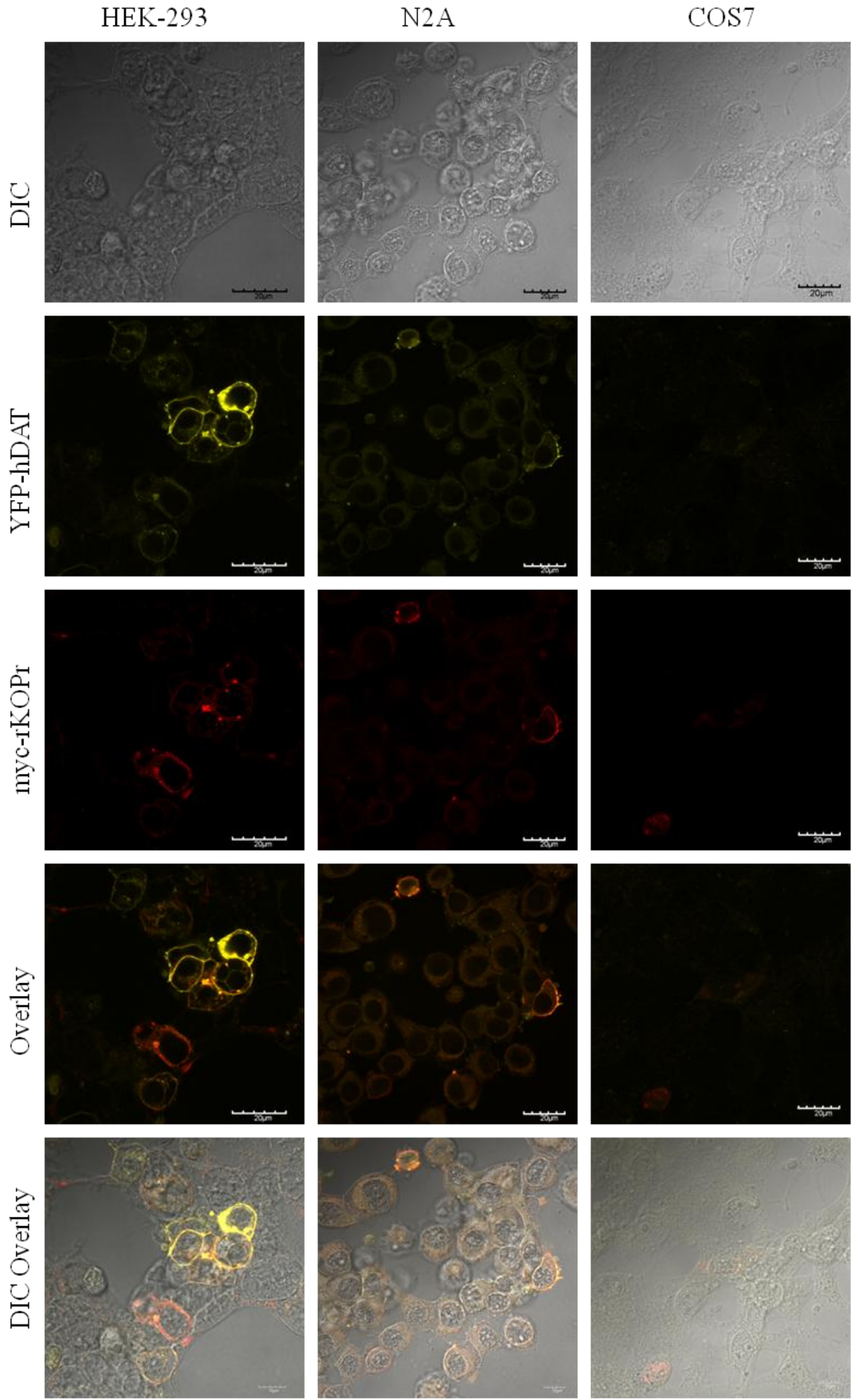

Figure 4.3: Co-expression of YFP-hDAT and myc-rKOPr in cell lines used in this study. HEK-293 (left column), N2A (middle column), and COS7 (right column) were stained for myc-rKOPr and imaged for YFP-hDAT and Cy5-myc-rKOPr to investigate co-expression of the proteins. YFP-hDAT and myc-rKOPr were co-expressed to varying degrees in each cell in all three cell lines, though expression levels were very low in COS7 cells. Expression levels of YFP-hDAT and myc-rKOPr varied between cells. 


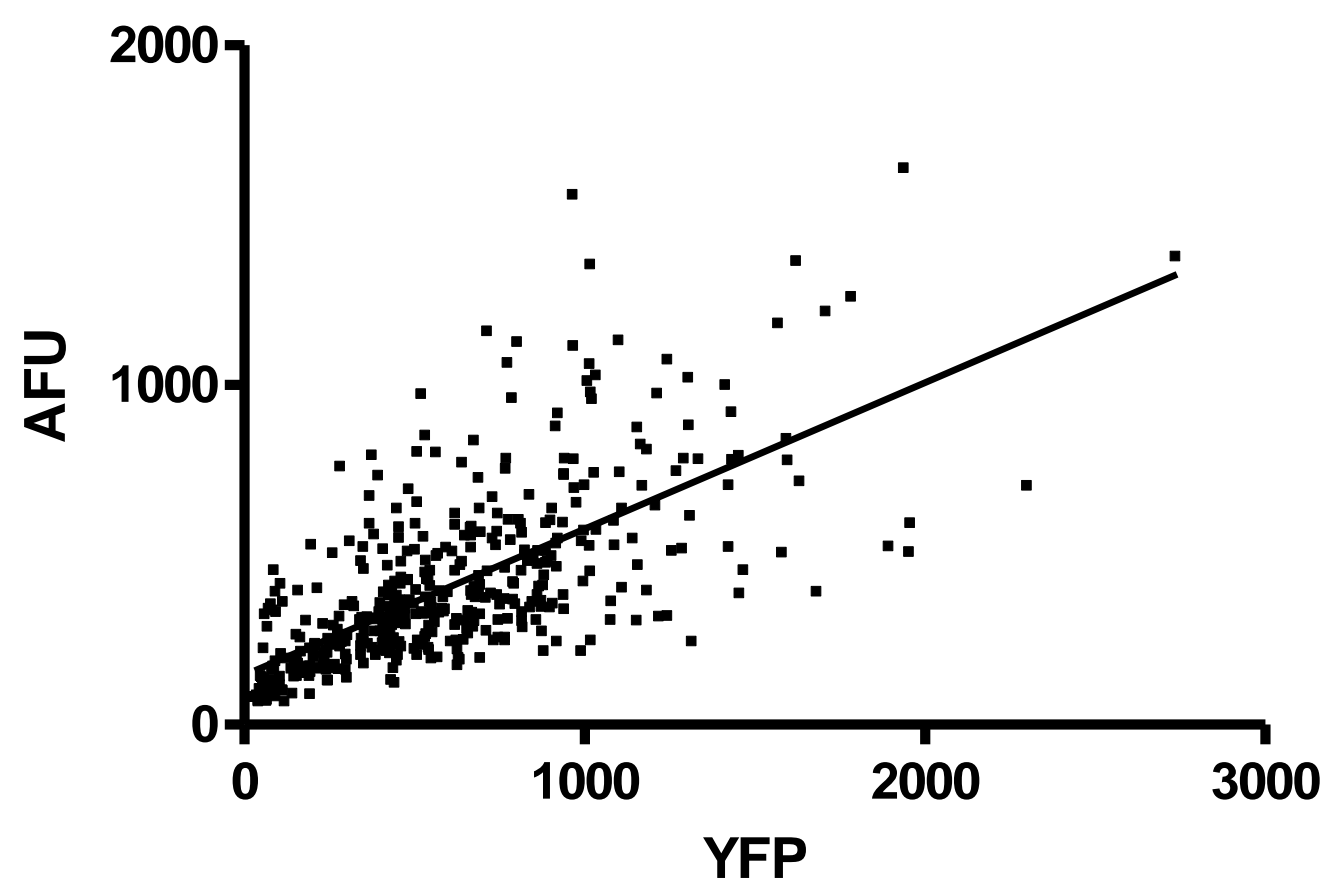

Figure 4.4: $\mathrm{ASP}^{+}$uptake and YFP intensity have a positive correlation in HEK293 cells. Increases in YFP-hDAT expression showed increased $\mathrm{ASP}^{+}$accumulation at $250 \mathrm{~s}\left(\mathrm{r}^{2}=0.4628, \mathrm{p}<0.001\right.$ compared to 0$)$. 

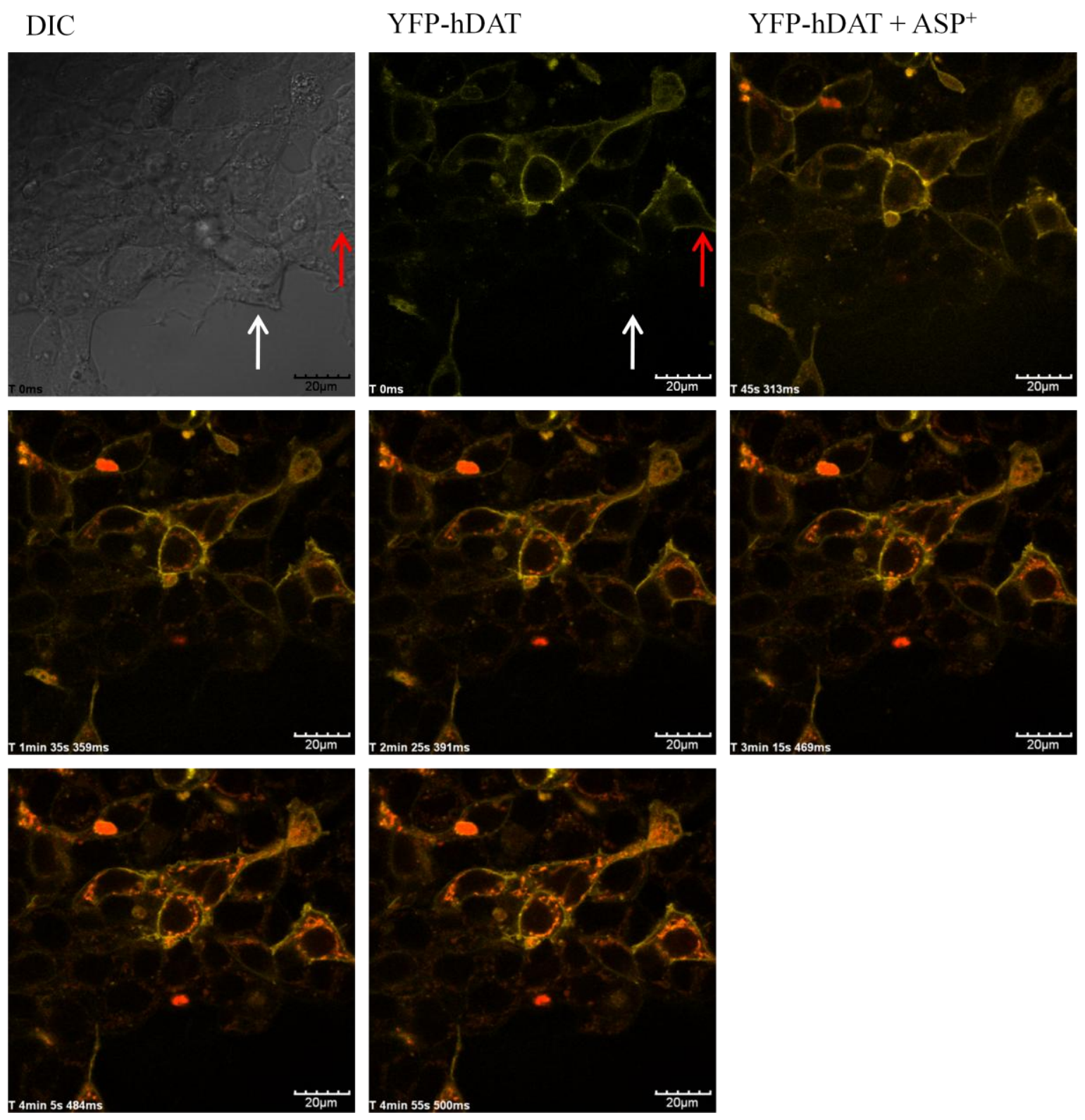

Figure 4.5: Time course of ASP+ uptake by HEK-293 cells transiently transfected with YFP-hDAT and myc-rKOPr (see DVD for movie). Note the transfected (yellow) cells (containing both YFP-hDAT and myc-rKOPr and indicated by a red arrow) take up greater amounts of $\mathrm{ASP}^{+}$(red) compared to non-transfected cells (indicated by a white arrow). Once $\mathrm{ASP}^{+}$is internalised it binds to the mitochondria within the cell. 
Table 4.1: Time taken for compounds to have their maximal effect on $\mathrm{ASP}^{+}$uptake rate (Appendix 2)

\begin{tabular}{|l|l|}
\hline KOPr agonist & Time taken for maximal effect (s) \\
\hline U50,488H & $100-160$ \\
\hline Salvinorin A & $60-120$ \\
\hline DS-1-240 & $200-260$ \\
\hline DS-3-216 & $200-260$ \\
\hline
\end{tabular}




\subsubsection{Acute changes in the function of DAT due to treatment with KOPr agonists}

In order to investigate the effects of $\mathrm{U} 50,488 \mathrm{H}$, salvinorin $\mathrm{A}$, and salvinorin $\mathrm{A}$ analogues on DAT function, $\mathrm{ASP}^{+}$uptake was measured (Figure 4.6). U50,488H, salvinorin A, DS-1-240, and DS-3-214 all caused a concentration-dependent increase of $\mathrm{ASP}^{+}$uptake into HEK-293 cells. Salvinorin A showed the largest effect, with $10 \mu \mathrm{M}$ causing a $40 \%$ increase in uptake $(\mathrm{p}<0.001)$; whereas, its two analogues showed effects more similar to $\mathrm{U} 50,488 \mathrm{H}$ with $10 \mu \mathrm{M}$ causing an increase in uptake of around $20 \%$ ( $\mathrm{p}<0.001$ for $\mathrm{U} 50,488 \mathrm{H}$ and DS-1-240, and $\mathrm{p}<0.01$ for DS-3-216). The KOPr antagonist nor-BNI $(1 \mu \mathrm{M})$ completely inhibited the increase caused by $10 \mu \mathrm{M}$ of all the compounds $(\mathrm{p}<0.001$ for $\mathrm{U} 50,488 \mathrm{H}$ and salvinorin A, and $\mathrm{p}<0.01$ for DS-1-240 and DS-3-216 compared to $10 \mu \mathrm{M})$. KOPr is a $\mathrm{G}_{0 / \mathrm{i}} \mathrm{G}$ protein-coupled receptor and is sensitive to PTX; therefore, the stimulatory effect of the agonists should be blocked by PTX. PTX (100 ng/mL) inhibited the effects of $10 \mu \mathrm{M}$ of all of the compounds studied, indicating that the effects of the compounds were due to KOPr activation $(p<0.001$ for $\mathrm{U} 50,488 \mathrm{H}$ and salvinorin $\mathrm{A}$, and $\mathrm{p}<0.01$ for Ds-1-240 and DS-3-216, One-way ANOVA followed by Bonferroni post test; $n=46-64)$. Addition of the vehicle control DMSO rather than KOPr agonists led to a non-significant decrease in the rate of $\mathrm{ASP}^{+}$ uptake, which is most likely due to photo-bleaching or the movement of the cells out of the focal plane over time. Neither nor-BNI nor PTX had an effect on $\mathrm{ASP}^{+}$uptake on their own (Figure 4.7). 

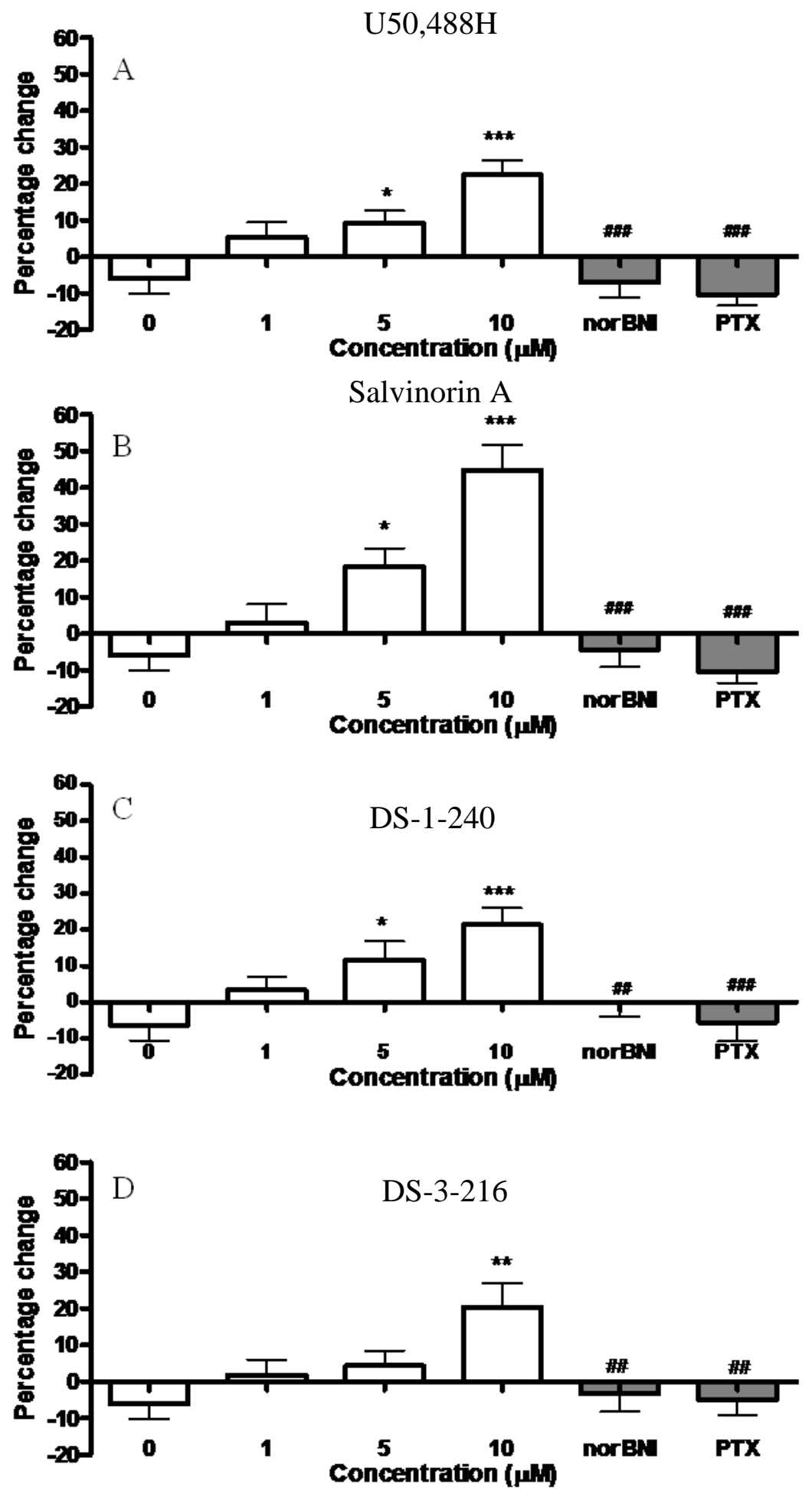

Figure 4.6: Changes in $\mathrm{ASP}^{+}$uptake after $\mathrm{KOPr}$ agonist addition is concentrationdependent and inhibited by nor-BNI and pertussis toxin. U50,488H (A), salvinorin A (B), DS-1-240 (C), and DS-3-216 (D) caused concentration-dependent increases in $\mathrm{ASP}^{+}$uptake rate, measured at the time of maximum uptake rate. This effect (at $10 \mu$ ) was significantly reversed by the addition of the KOPr antagonist nor-BNI $(1 \mu \mathrm{M})(\mathrm{n}=$ 46-64 cells). The addition of DMSO (0) as the vehicle control did not lead to a significant decrease in uptake from 0 for any of the agonists (One sample Student's ttest). Experiments were carried out over 3 transfections with at least 2 dishes used per transfection. $* \mathrm{p}<0.05, * * \mathrm{p}<0.01, * * * \mathrm{p}<0.001$ compared to the vehicle control without drug (0). ${ }^{\# \#} \mathrm{p}<0.01,{ }^{\# \#} \mathrm{p}<0.001$ compared to $10 \mu \mathrm{M}$ drug. One-way ANOVA followed by a Bonferroni post test. 


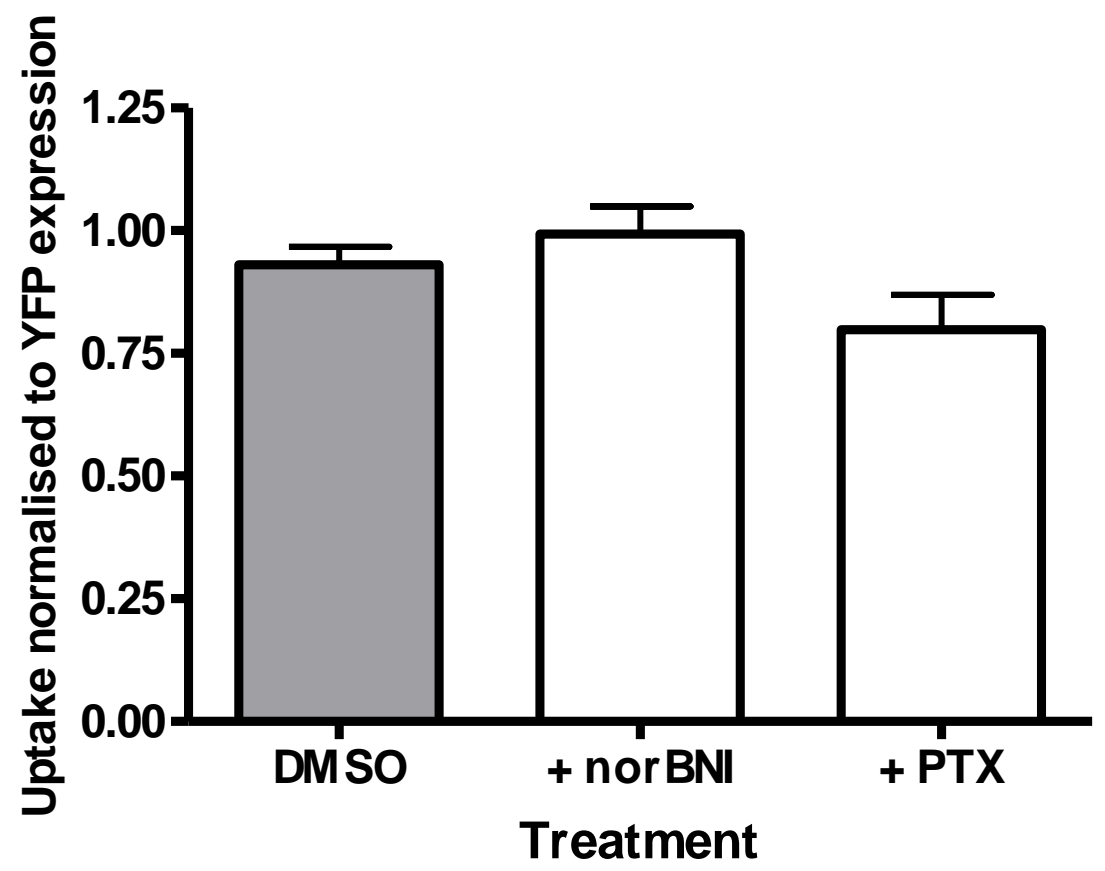

Figure 4.7: nor-BNI and PTX have no effect on uptake of $\mathrm{ASP}^{+}$on their own. Incubation of HEK-293 cells with nor-BNI for $30 \mathrm{~min}$ and PTX for 16-24 h had no effect on $\mathrm{ASP}^{+}$uptake at $200 \mathrm{~s}$ after $\mathrm{ASP}^{+}$addition compared to control cells $(\mathrm{n}=31-47$ cells, over three transfections with at least 2 dishes used per transfection, One-way ANOVA followed by Bonferroni post test). 


\subsubsection{The effect of KOPr agonists in different cell lines}

To confirm the results seen in HEK-293 cells, the KOPr agonists were also screened in another kidney cell line, COS7 (green monkey kidney cells) and also a neuronal cell line, N2A (mouse neuroblastoma cells). Both cell lines showed a positive correlation between YFP-hDAT expression and $\mathrm{ASP}^{+}$uptake (Figure 4.8 and Figure 4.9). Addition of $10 \mu \mathrm{M}$ of the agonists caused a significant increase in $\mathrm{ASP}^{+}$uptake in both cell lines of between 10 and $25 \%$, and this effect was blocked by the addition of nor-BNI $(1 \mu \mathrm{M})$ and PTX (100 ng/mL) (Figure 4.8f and Figure 4.9f). Neither nor-BNI nor PTX caused changes in uptake themselves (Figure 4.8 and Figure 4.9). While the increases in uptake were significant, they were less than the effect seen in HEK-293 cells. These data confirm the results with HEK-293 cells and suggest that HEK-293 cells, which show a larger $\mathrm{ASP}^{+}$effect than the other two cell lines and are much easier to transfect, are a good model for measuring DAT function in isolated cells. 


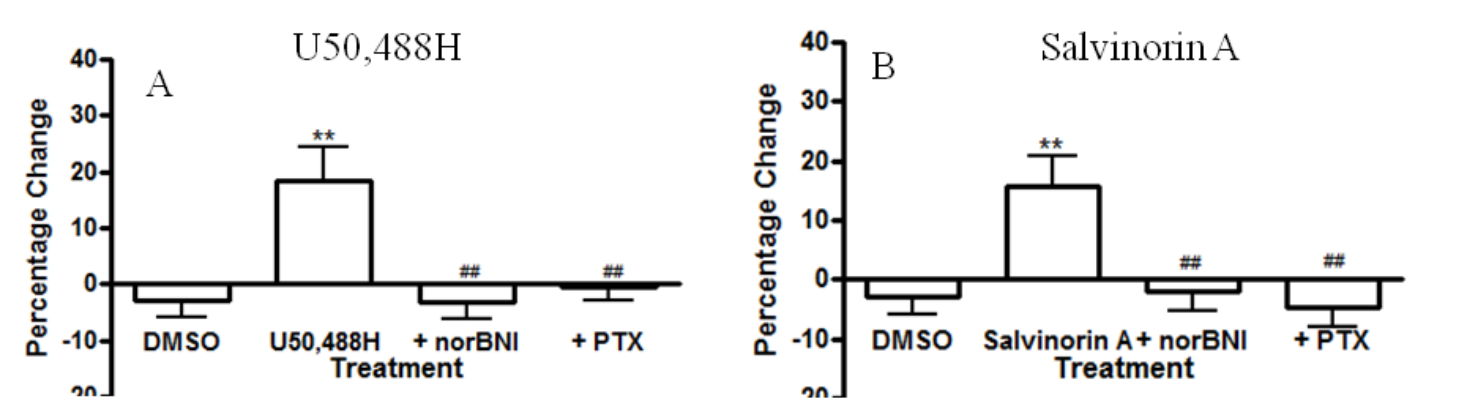

DS-1-240
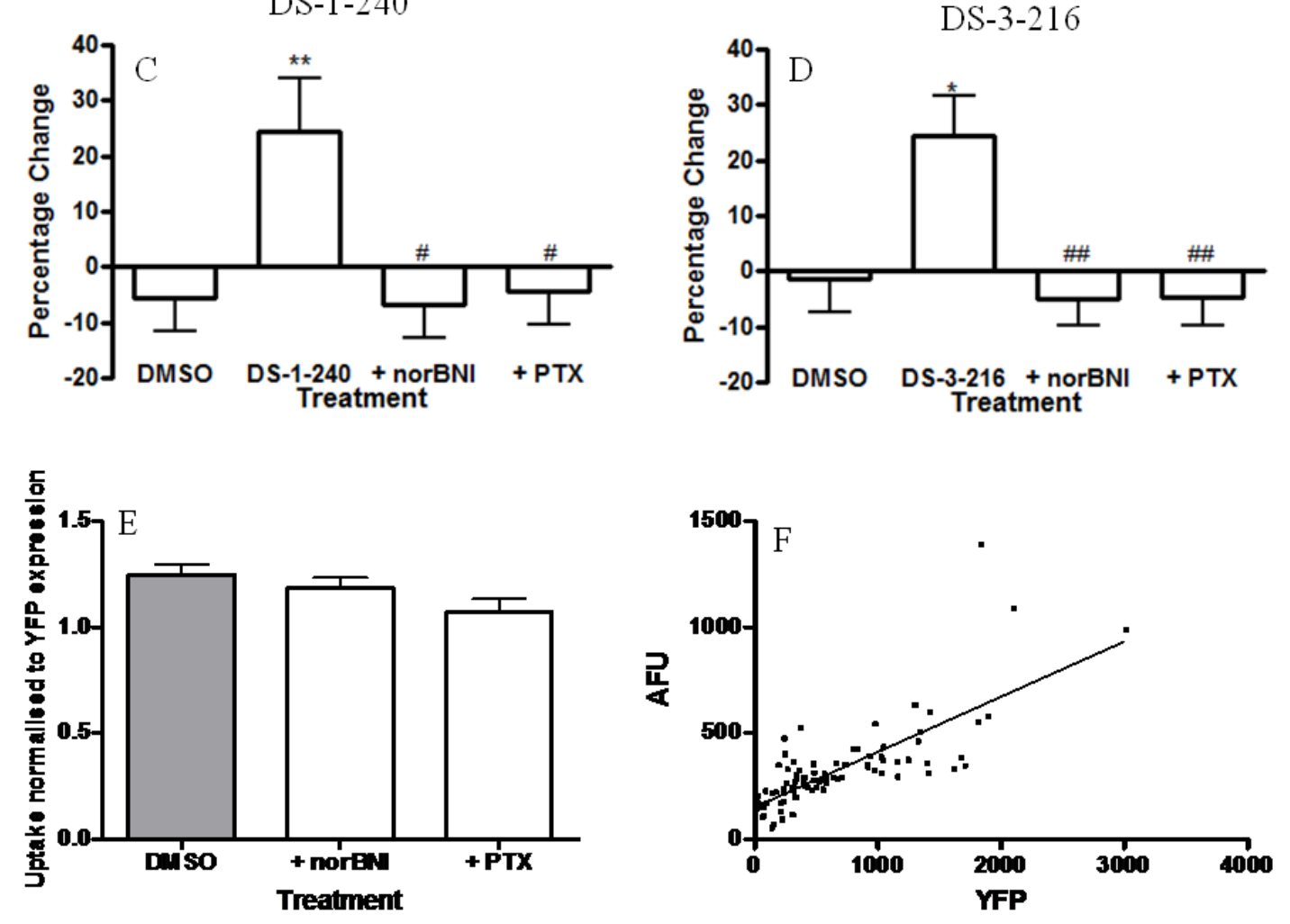

Figure 4.8: Addition of $10 \mu \mathrm{M}$ of KOPr agonists to COS7 cells causes an increase in $\mathrm{ASP}^{+}$uptake that is reversible by nor-BNI and PTX. U50,488H (A), salvinorin A (B), DS-1-240 (C), and DS-3-216 (D) $(10 \mu \mathrm{M})$ all caused significant increases in DAT function in COS7 cells. This effect was significantly blocked by the addition of norBNI $(1 \mu \mathrm{M}, 30 \mathrm{~min})$ and PTX (100 $\mathrm{ng} / \mathrm{ml}, 16-24 \mathrm{~h})$. The addition of DMSO as the vehicle control did not lead to a significant decrease in uptake from 0 for any of the agonists (One sample Student's t-test). nor-BNI and PTX alone did not cause changes in the uptake of $\mathrm{ASP}^{+}(\mathrm{E})$. There was a positive correlation between YFP-hDAT expression and $\mathrm{ASP}^{+}$uptake in COS7 cells $\left(\mathrm{R}^{2}=0.5774\right)$ that was significantly different from $0(\mathrm{p}<0.0001) . \quad \mathrm{n}=25-35$, experiments were carried out over 3 transfections with at least 3 dishes used per transfections. $* \mathrm{p}<0.05, * * p<0.01$, $* * * p<0.001$ compared to DMSO control. ${ }^{\#} \mathrm{p}<0.01,{ }^{\# \#} \mathrm{p}<0.001$ compared to $10 \mu \mathrm{M}$. One-way ANOVA followed by a Bonferroni post test. 

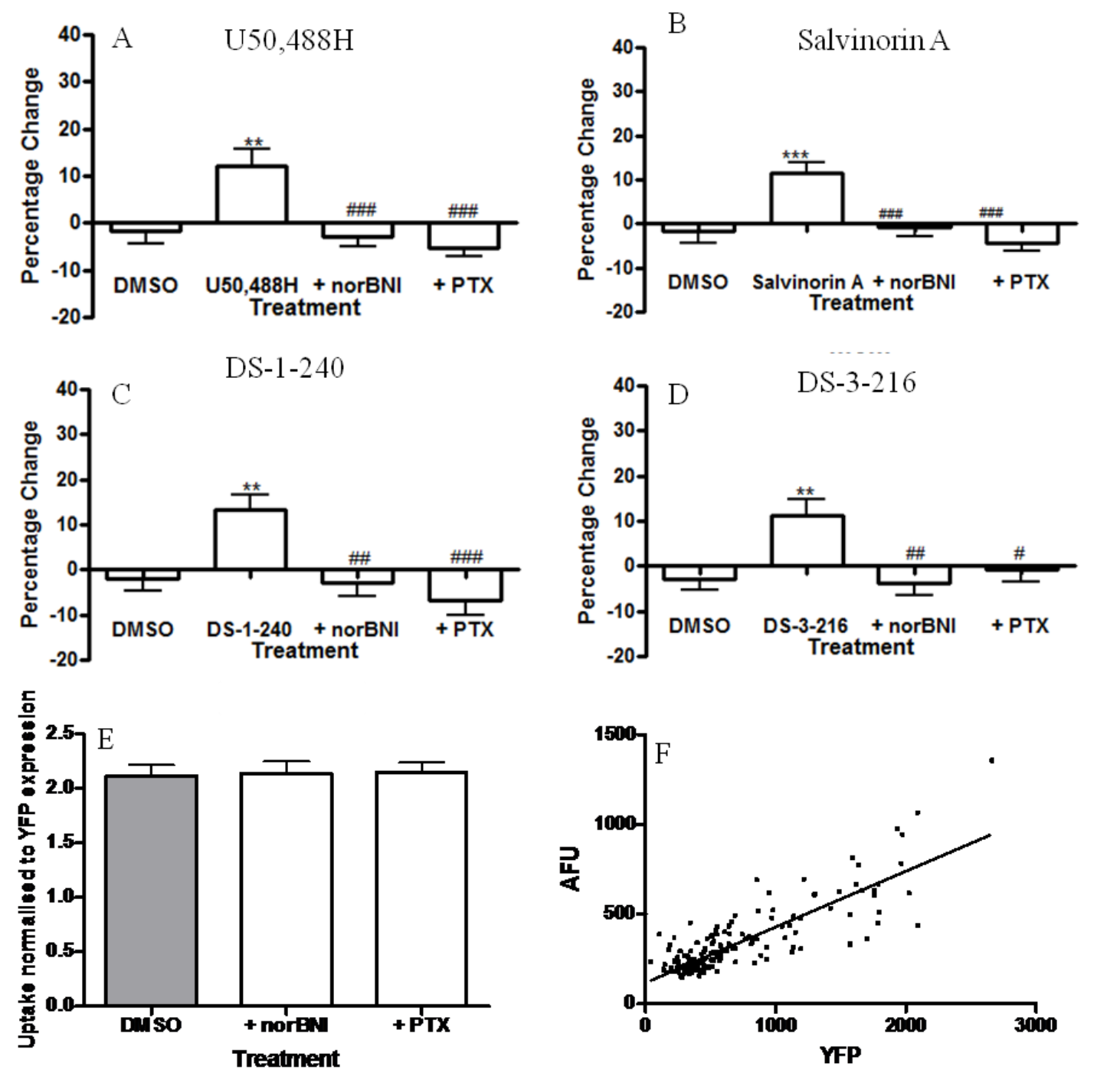

Figure 4.9: Addition of $10 \mu \mathrm{M}$ of $\mathrm{KOPr}$ agonists to $\mathrm{N} 2 \mathrm{~A}$ cells causes an increase in $\mathrm{ASP}^{+}$uptake that is reversible by nor-BNI and PTX. U50,488H (A), salvinorin A (B), DS-1-240 (C) and DS-3-216 (D) $(10 \mu \mathrm{M})$ caused a significant increase in DAT function in N2A cells. This effect was significantly blocked by the addition of nor-BNI $(1 \mu \mathrm{M}, 30 \mathrm{~min})$ and PTX (100 ng/ml, 16-24 h). The addition of DMSO as a vehicle control did not lead to a significant decrease in uptake from 0 for any of the agonists (One sample Student's t test). nor-BNI and PTX alone did not cause changes in the uptake of $\mathrm{ASP}^{+}(\mathrm{E})$. There was a positive correlation between YFP-hDAT expression and $\mathrm{ASP}^{+}$uptake seen in these cells $\left(\mathrm{R}^{2}=0.7054\right)$ which was significantly different from the DMSO control $(\mathrm{p}<0.0001) . \mathrm{n}=25-35$, experiments were carried out over 3 transfections with at least 3 dishes used per transfection. $* * p<0.01$, ***p $<0.001$ compared to the DMSO control. ${ }^{\#} \mathrm{p}<0.01,{ }^{\# \# \# ~}<0.001$ compared to $10 \mu \mathrm{M}$. One-way ANOVA followed by a Bonferroni post test. 


\subsubsection{The effect of a 30 min incubation with $\mathrm{KOPr}$ agonists on $\mathrm{ASP}^{+}$uptake}

To investigate whether the KOPr agonists had a prolonged effect on DAT function, the effect on $\mathrm{ASP}^{+}$uptake of a 30 min incubation of HEK-293 cells with KOPr agonists was measured. This experiment is a between-cell design, and makes comparisons between groups of cells incubated with either vehicle or KOPr agonist. Cells were incubated for $30 \mathrm{~min}$ with the $\mathrm{KOPr}$ agonist $(10 \mu \mathrm{M})$, and then $\mathrm{ASP}^{+}$containing the KOPr agonist was added and uptake was measured over $5 \mathrm{~min}$. The total $\mathrm{ASP}^{+}$uptake after $5 \mathrm{~min}$ was then normalised to YFP-hDAT expression and compared across treatment groups. These experiments were carried out using two sets of $\mathrm{ASP}^{+}$stock and are therefore presented separately. The control graphs (Figure 4.10c and d) represent the average YFP-hDAT expression over the different treatment groups, and these values did not differ significantly. $\mathrm{U} 50,488 \mathrm{H}$ and salvinorin $\mathrm{A}$ both caused a significant increase in $\mathrm{ASP}^{+}$uptake after 30 min (Figure 4.10a, $\mathrm{p}<0.05$ and $\mathrm{p}<0.01$, respectively); whereas, DS-1-240 and DS-3-2216 had no effect (Figure 4.10b), although the decrease seen with DS-1-240 was almost significant (Student's t-test, $\mathrm{p}=0.0861$ ). 

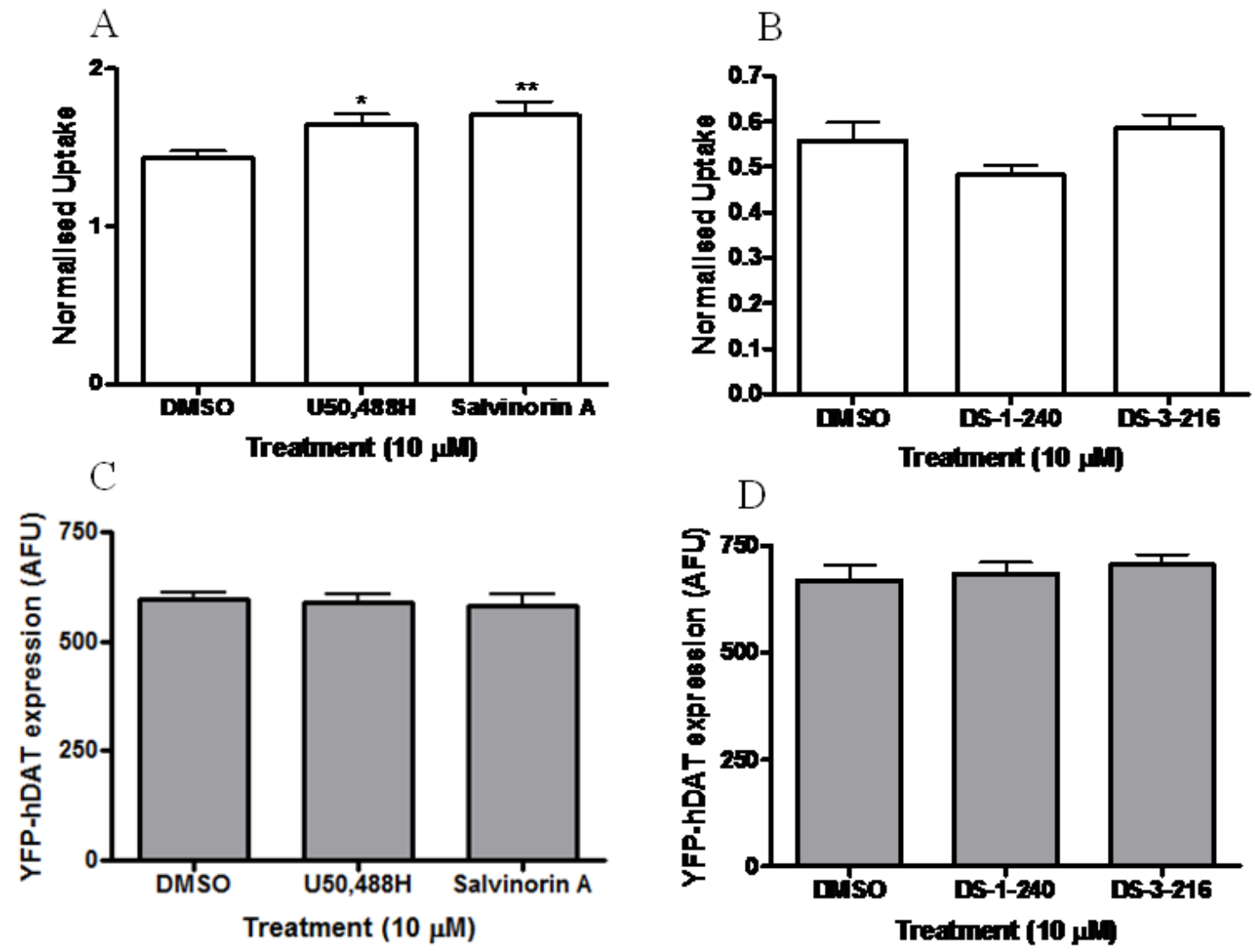

Figure 4.10: Long-term effects of KOPr agonists on $\mathrm{ASP}^{+}$uptake in HEK-293 cells. A) $\mathrm{U} 50,488 \mathrm{H}$ and salvinorin A significantly increased $\mathrm{ASP}^{+}$uptake after $30 \mathrm{~min}$; whereas, B) DS-1-240 and DS3-216 had no significant effect. C,D) YFP-hDAT expression was consistent over the replicates. $\mathrm{n}=30-40$ cells over 3 transfections with a minimum of 2 dishes per transfection. One-way ANOVA followed by a Bonferonni post test, $* \mathrm{p}<0.05, * * \mathrm{p}<0.01$. 


\subsubsection{Acute cell surface expression studies}

\subsubsection{ASP $^{+}$binding studies}

To investigate whether the rapid increase in function observed following KOPr agonist addition was caused by an increase in the cell surface expression of YFP-hDAT, ASP ${ }^{+}$ binding studies, cell surface biotinylation, and TIRFM were carried out on HEK-293 cells. $\mathrm{ASP}^{+}$binding studies were carried out on cells that had been incubated in the test $\mathrm{KOPr}$ agonist for the time required to give the maximal functional change in $\mathrm{ASP}^{+}$ uptake (Table 4.1). Medium containing the test compound was then replaced with $\mathrm{ASP}^{+}$containing the test compound, and 1 min of data was collected, taking images with the shortest wait between them (about $1.7 \mathrm{~s}$ ). The slope of the line was analysed from 3 to $7 \mathrm{~s}$ and this was normalised to YFP-hDAT expression (Figure 4.11b). Data showed that there was a non-significant increase seen with $\mathrm{U} 50,488 \mathrm{H}$, salvinorin $\mathrm{A}$, and DS-3-216, and a significant increase of $\mathrm{ASP}^{+}$binding to YFP-hDAT after DS-1-240 treatment. 

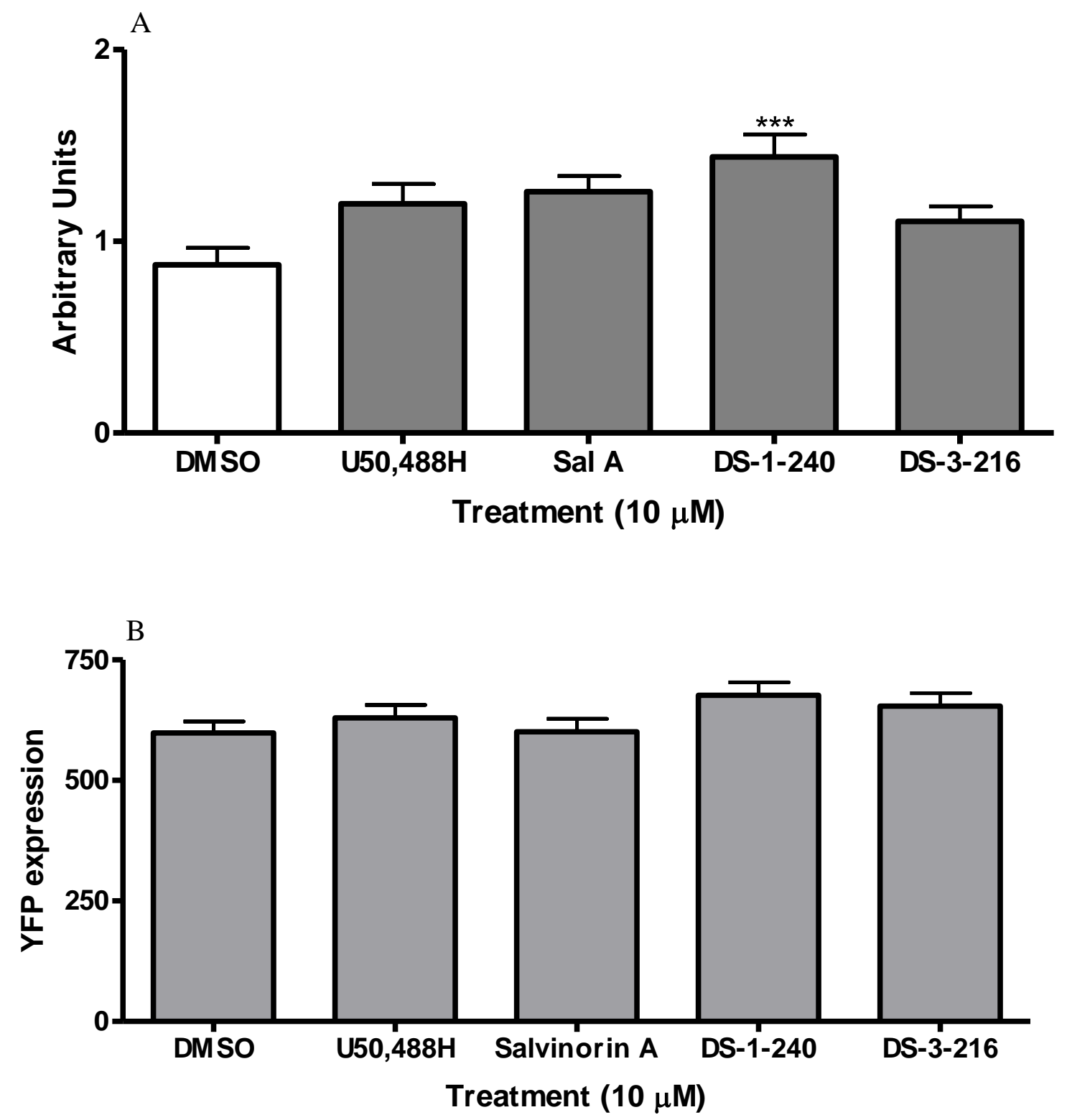

Figure 4.11: KOPr agonists cause no changes in binding of $\mathrm{ASP}^{+}$to YFP-hDAT. (A) Incubation of cells with KOPr agonists for the time required to give a maximal effect caused a non-significant increase in $\mathrm{ASP}^{+}$binding to YFP-hDAT for U50,488H, salvinorin A, and DS-3-216 and a significant increase in binding for DS-1-240. B) There was no significant difference between the YFP-hDAT expression of the cells analysed between groups. $n=36-52$ over 4 transfections with a minimum of 2 plates per transfection. $* * * \mathrm{p}<0.001$, one-way ANOVA followed by a Bonferroni post test. 


\subsubsection{Cell surface biotinylation studies}

Cell surface biotinylation studies were also carried out to determine whether the changes in function seen by $\mathrm{ASP}^{+}$uptake were due to changes in cell surface expression of YFP-hDAT. Initial experiments were carried out with cells incubated for the time taken for the compounds to have their maximal functional effect (salvinorin A: $60 \mathrm{~s}$, U50,488H and U69,593: $100 \mathrm{~s}, \mathrm{DS}-1-240$ and DS-3-216: $200 \mathrm{~s}$ ) (Figure 4.12a). The classic KOPr agonist U69,593 was also used in this study, and the time to maximal effect measured for U50,488H was selected. As U69,593 was dissolved in absolute ethanol rather than DMSO, it was presented in a separate graph with its own vehicle (Figure 4.12b). After the gels were scanned for YFP-hDAT, they were immunoblotted for GAPDH, which is found only intracellularly in HEK-293 cells, to confirm that the cells had not been lysed before the biotin had been added which would lead to false positives. There was no detectable increase in cell surface expression of YFP-hDAT at these time points for any of the compounds studied (Figure 4.12a and b) and no GAPDH was present in any of the cell surface fractions measured.

\subsubsection{Total internal reflection fluorescence microscopy}

To confirm either the positive result gained from the $\mathrm{ASP}^{+}$binding assays or the negative result gained from the cell surface biotinylation assays, the more sensitive technique, TIRFM, was also conducted at the early time points. TIRFM experiments were run for $300 \mathrm{~s}$ with addition of the compound occurring at $100 \mathrm{~s}$ and images taken every $5 \mathrm{~s}$. The results confirmed cell surface biotinylation data as no significant changes in YFP-hDAT cell surface density were found for any of the KOPr agonists (Figure 4.14a-e).

Overall these data suggest that the initial increase in transport function of YFP-hDAT after KOPr activation is not due to increases in cell surface expression of YFP-hDAT, but as there was an increase in $\mathrm{ASP}^{+}$binding to the cells it suggests that there is an increase in active YFP-hDAT on the cell surface. 


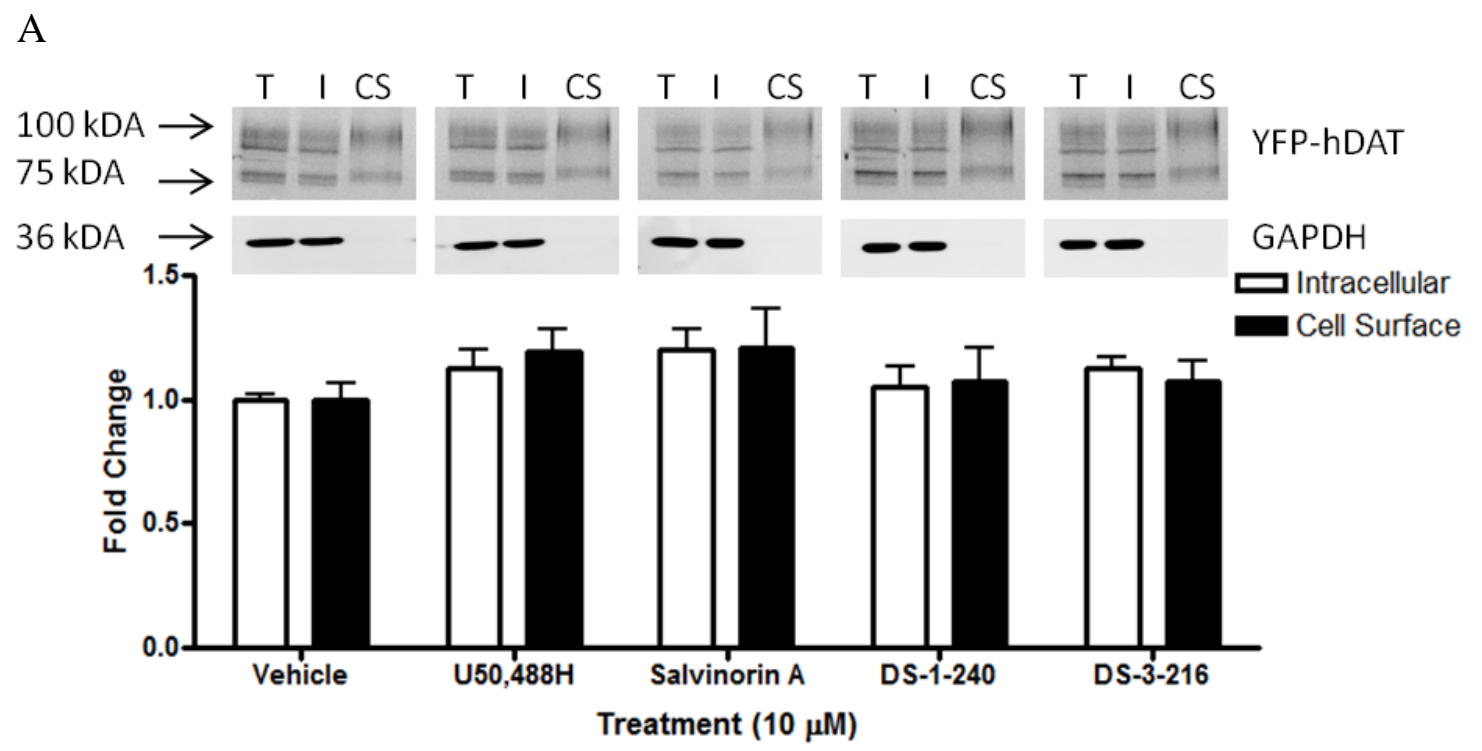

$\mathrm{B}$

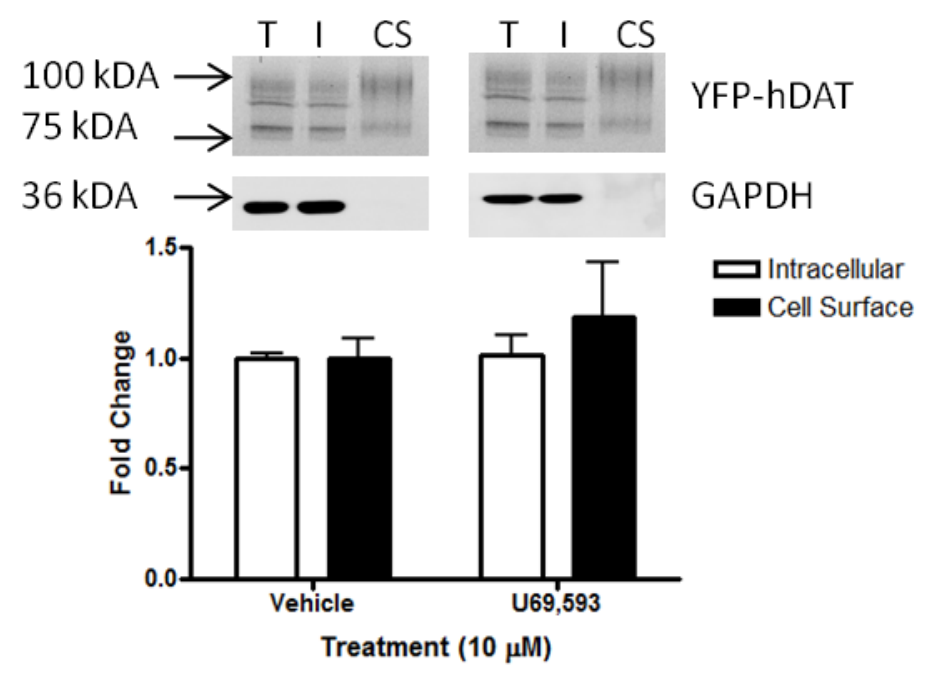

Figure 4.12: KOPr agonists do not alter cell surface expression of YFP-hDAT at short time points. Cells were treated for the time taken for the agonists to have their maximal functional effect and then cell surface biotinylated. There was no detectable change in cell surface expression after agonist treatment for any compound tested. Shown is a representative gel for YFP-hDAT and Western blot for GAPDH respectively. $\mathrm{T}=$ total fraction, $\mathrm{I}=$ internal fraction, $\mathrm{CS}=$ cell surface fraction $(\mathrm{n}=8-11$ preparations). Data are presented as average \pm SEM. One-way ANOVA followed by Bonferroni post test showed no significant differences from the control. 

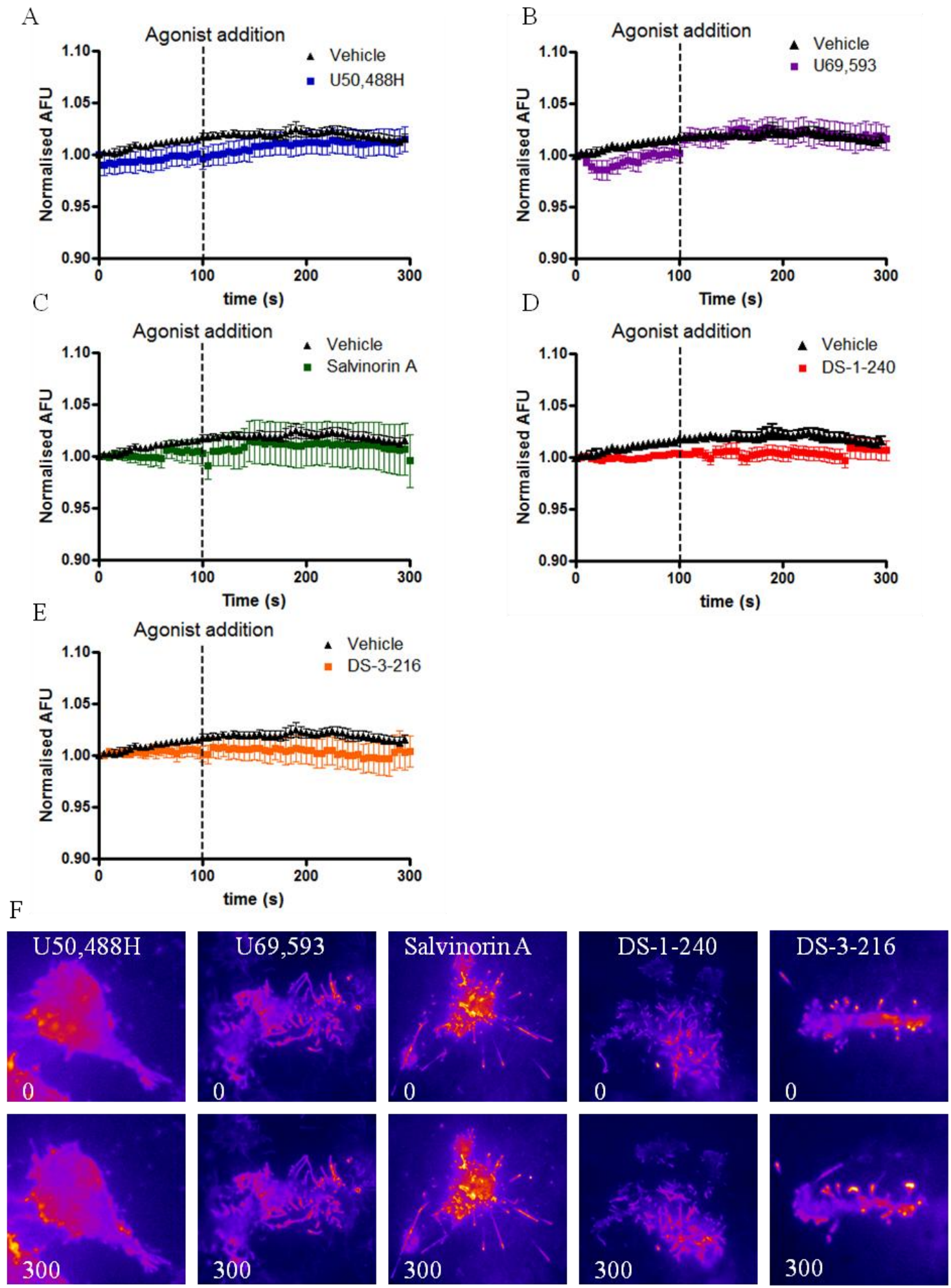

Figure 4.13: KOPr agonists do not alter cell surface expression of YFP-hDAT at short time points as measured by TIRFM. U50,488H (A), U69,593 (B), salvinorin A (C), DS-1-240 (D), and DS-3-216 (E) did not alter cell surface expression of YFPhDAT at $200 \mathrm{~s}$ after drug treatment $(\mathrm{n}=12-16)$. Shown are representative images taken at time 0 and $300 \mathrm{~s}$ for each treatment $(\mathrm{F})$. Two-way ANOVA followed by Bonferroni post test. 
4.4.5 Chronic effects of KOPr agonists on cell surface expression of YFP-hDAT As there were changes in uptake at 30 min with $\mathrm{U} 50,488 \mathrm{H}$ and salvinorin A (Figure 4.10), the effect of a 30 min incubation with all of the test compounds on cell surface expression of YFP-hDAT was investigated using TIRFM and cell surface biotinylation. Using cell surface biotinylation, a 30 min incubation with salvinorin A (Figure 4.16), U69,593 (Figure 4.15) and DS-3-216 (Figure 4.18) all caused increased cell surface expression of YFP-hDAT. Significant increases of $25 \% \pm 11.5 \%$ (salvinorin A), $37 \% \pm$ $14.7 \%$ (U69,593), and 23\% $\pm 7.3 \%$ (DS-3-216) were obtained. TIRFM studies were conducted over a period of $40 \mathrm{~min}$, with one image taken every minute. The test compound was added after $10 \mathrm{~min}$ of baseline recording, and a further $30 \mathrm{~min}$ of images were collected. TIRFM studies confirmed the increases in cell surface expression for salvinorin A and U69,593 with significant increases of $11.5 \% \pm 3.5 \%$ and $9.3 \% \pm 3 \%$, respectively. While DS-3-216 showed an increase of $6.1 \% \pm 2.1 \%$ after $30 \mathrm{~min}$ treatment, the increase was not significant. Interestingly, U50,488H (Figure 4.15) and DS-1-240 (Figure 4.18) had no effect on cell surface expression, suggesting there may be differences between how these compounds regulate DAT, or that any changes that may occur are too small for these techniques to measure. 
A
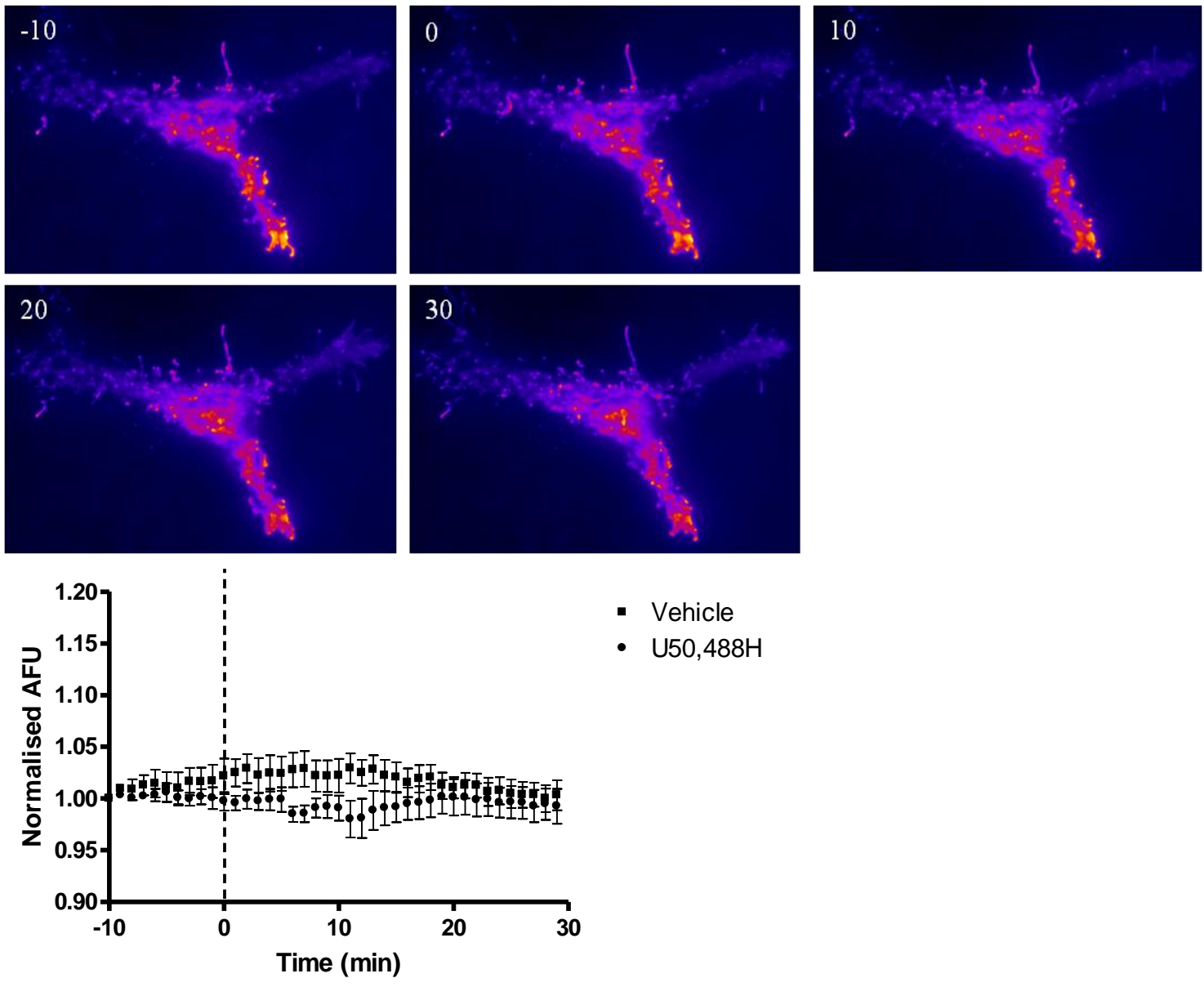

- Vehicle

- U50,488H

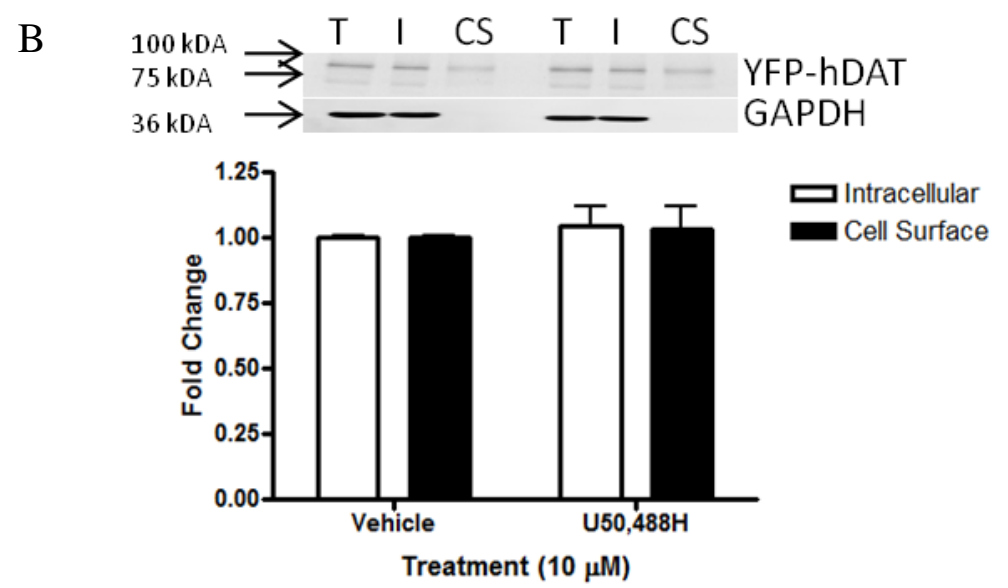

Figure 4.14: A 30 min incubation with $\mathrm{U50,488H}$ does not change cell surface expression of YFP-hDAT. (A) TIRFM studies were carried out on HEK-293 cells expressing YFP-hDAT and myc-rKOPr, with agonist addition occurring at 10 min and treatment lasting for $30 \mathrm{~min}$. There were no changes seen in cell surface expression of YFP-hDAT throughout the $30 \mathrm{~min}$ incubation, Two-way ANOVA followed by Bonferroni post test $(n=12-16)$. Shown is a representative cell with images taken at 10, 0, 10, 20, and $30 \mathrm{~min}$. (B) Cells were also incubated with U50,488H for $30 \mathrm{~min}$ before biotinylation studies were carried out. No changes were seen in cell surface expression of YFP-hDAT after $30 \mathrm{~min}$, one-way ANOVA followed by Bonferroni post test. $\mathrm{T}=$ total, $\mathrm{I}=$ internal, $\mathrm{CS}=$ cell surface. Values are mean $\pm \mathrm{SEM}(\mathrm{n}=9-13)$. 
A
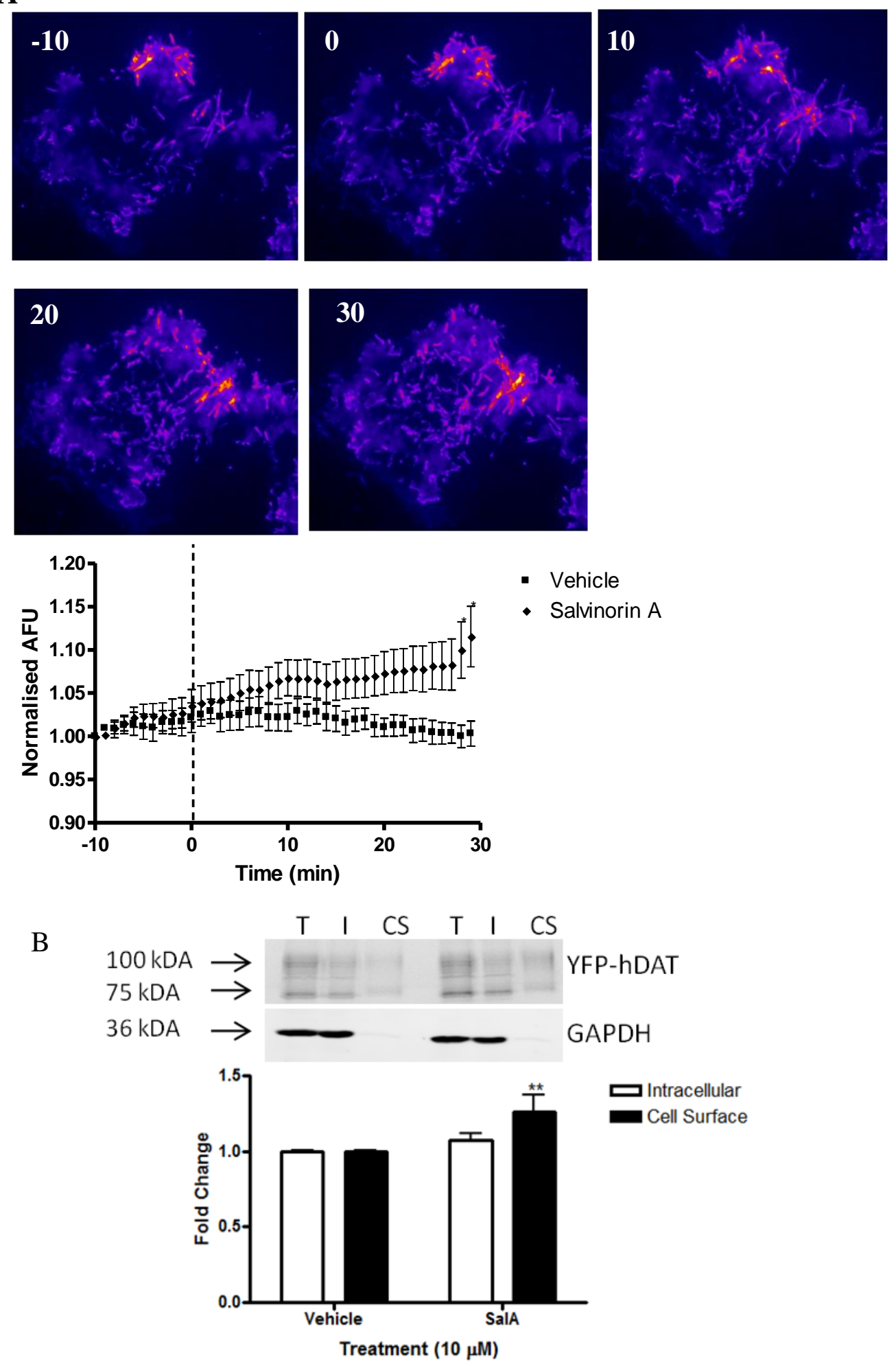

Figure 4.15: A 30 min incubation with salvinorin A led to a significant increase in cell surface expression of YFP-hDAT. (A) TIRFM studies showed that after a 29 min incubation with salvinorin A, a significant increase in YFP-hDAT was seen. Two-way ANOVA followed by Bonferroni Post test, $* p<0.05$. A representative cell is shown with images at $-10,0,10,20$, and $30 \mathrm{~min}$. $(n=7-9)$. (B) Cells were also incubated with salvinorin A for $30 \mathrm{~min}$ before cell surface biotinylation experiments. A significant increase in cell surface expression of YFP-hDAT was seen One-way ANOVA followed by Bonferroni Post test, $*^{*} \mathrm{p}<0.01 . \quad(\mathrm{n}=12-16) . \quad \mathrm{T}=$ total, $\mathrm{I}=$ internal, $\mathrm{CS}=$ cell surface. 
A
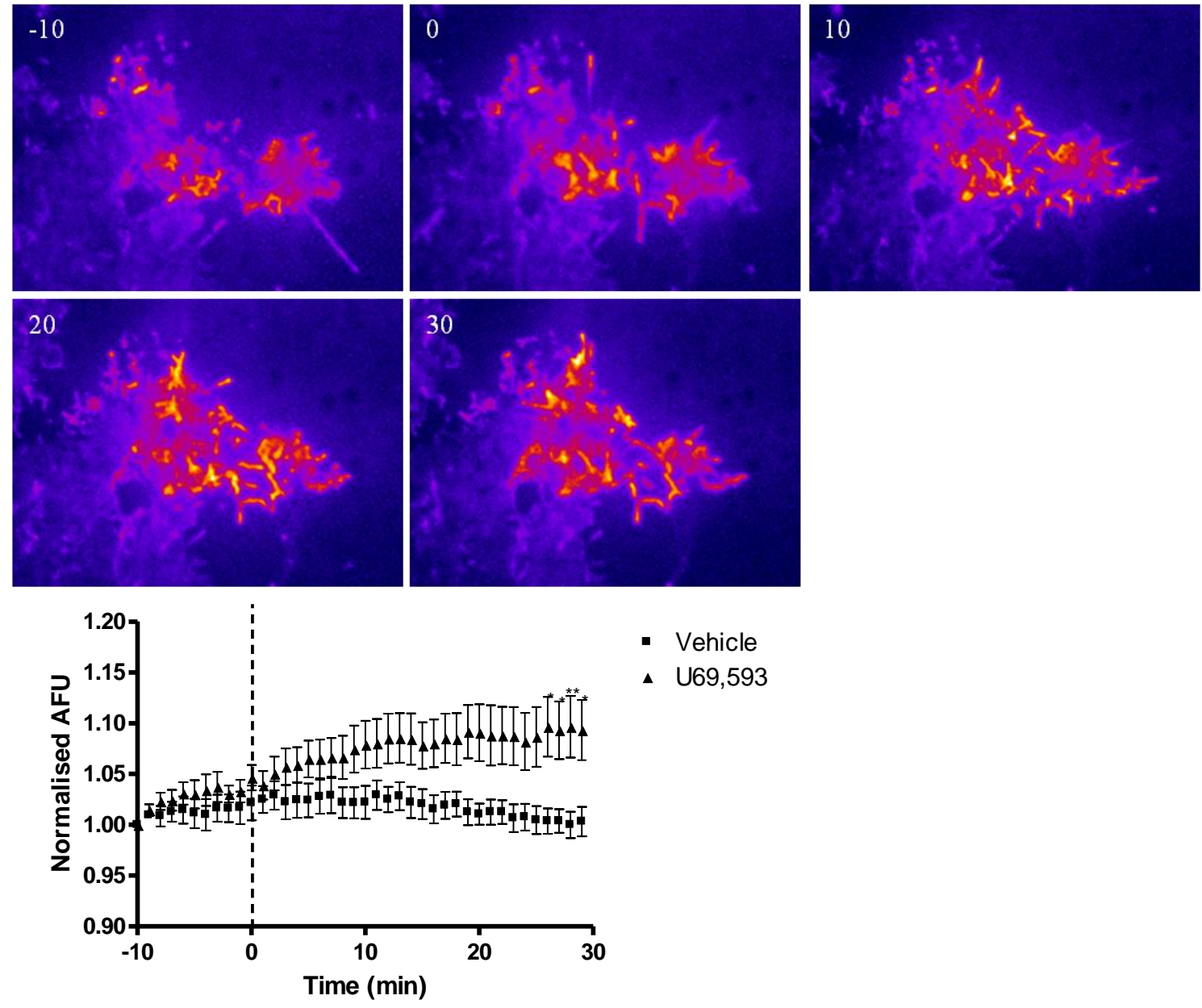

B
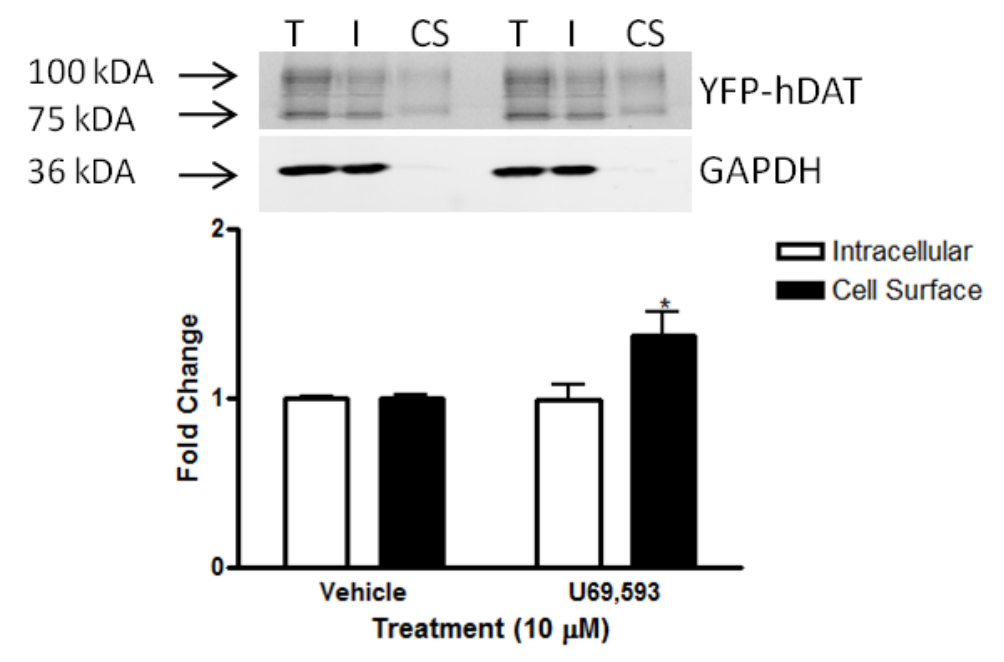

Figure 4.16: A 30 min incubation with U69,593 led to a significant increase in cell surface expression of YFP-hDAT. (A) TIRFM studies showed that after a $29 \mathrm{~min}$ incubation with U69,593 a significant increase in YFP-hDAT was seen. Two-way ANOVA followed by Bonferroni Post test, $* \mathrm{p}<0.05$, **p<0.01 (n = 12). A representative cell is shown with images at $-10,0,10,20$, and $30 \mathrm{~min}$. (B) Cells were also incubated with U69,593 for 30 min before cell surface biotinylation experiments. A significant increase in cell surface expression of YFP-hDAT was seen. One-way ANOVA followed by Bonferroni Post test, ${ }^{*} \mathrm{p}<0.05 .(\mathrm{n}=6-8) . \mathrm{T}=$ total, $\mathrm{I}=$ internal, $\mathrm{CS}=$ cell surface. 

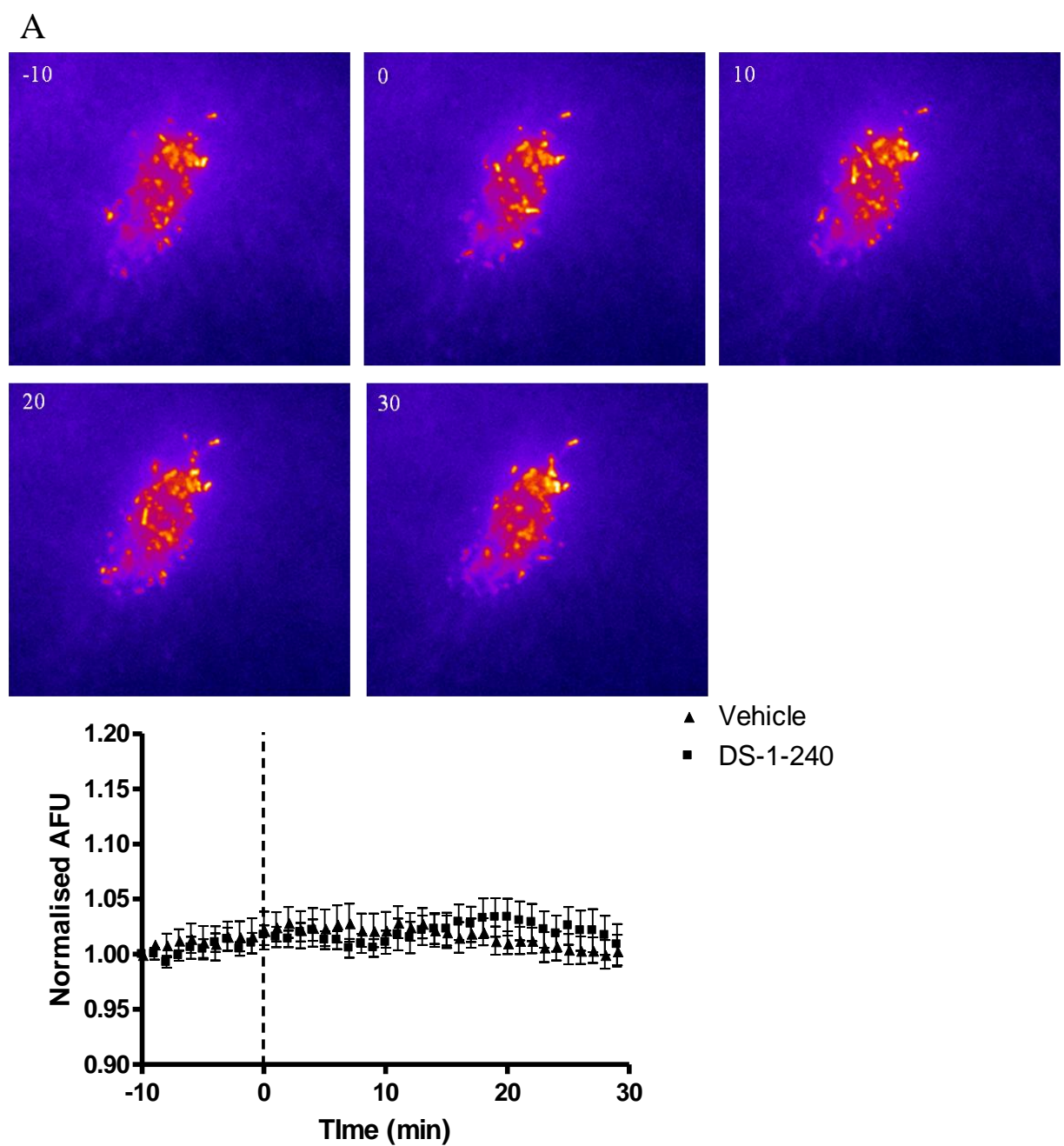

- Vehicle

- DS-1-240
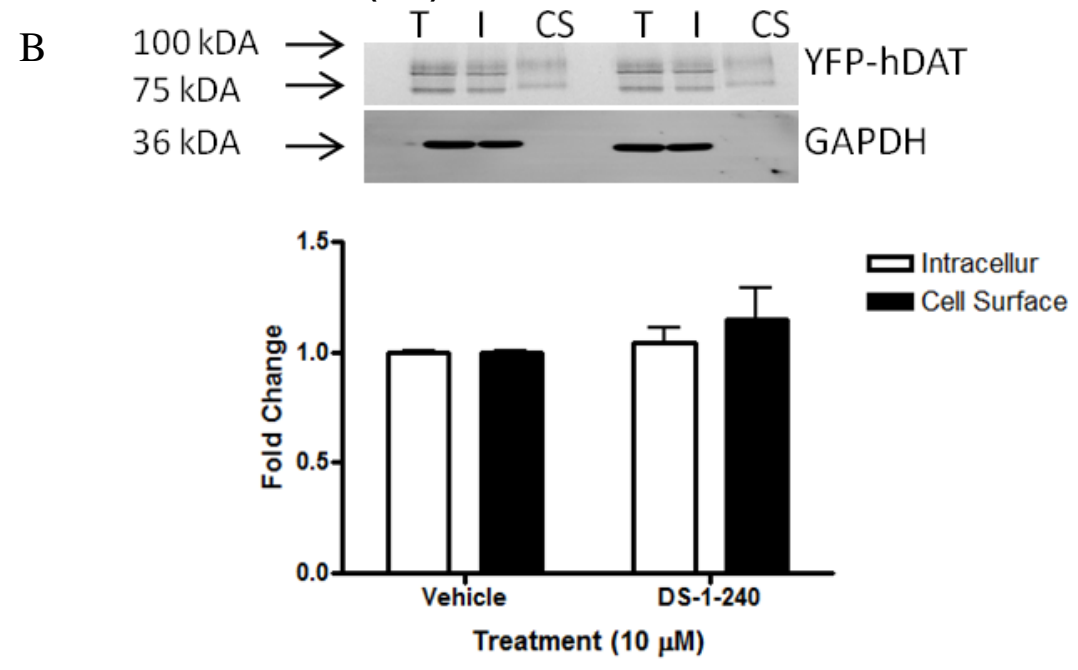

Figure 4.17: A 30 min incubation with DS-1-240 does not change cell surface expression of YFP-hDAT. (A) TIRFM studies were carried out on HEK-293 cells expressing YFP-hDAT and myc-rKOPr, with drug addition occurring at $10 \mathrm{~min}$. There were no changes seen in cell surface expression of YFP-hDAT throughout the $30 \mathrm{~min}$ incubation with the drug, two-way ANOVA followed by Bonferroni post test $(\mathrm{n}=9$ 10). Shown is a representative cell with images taken at $-10,0,10,20$, and $30 \mathrm{~min}$. (B) Cells were also incubated with DS-1-240 for $30 \mathrm{~min}$ before biotinylation studies were carried out. No changes were seen in cell surface expression of YFP-hDAT after 30 min, one-way ANOVA followed by Bonferroni post test $(\mathrm{n}=12-13)$. $\mathrm{T}=$ total, $\mathrm{I}=$ internal, $\mathrm{CS}=$ cell surface. 
A
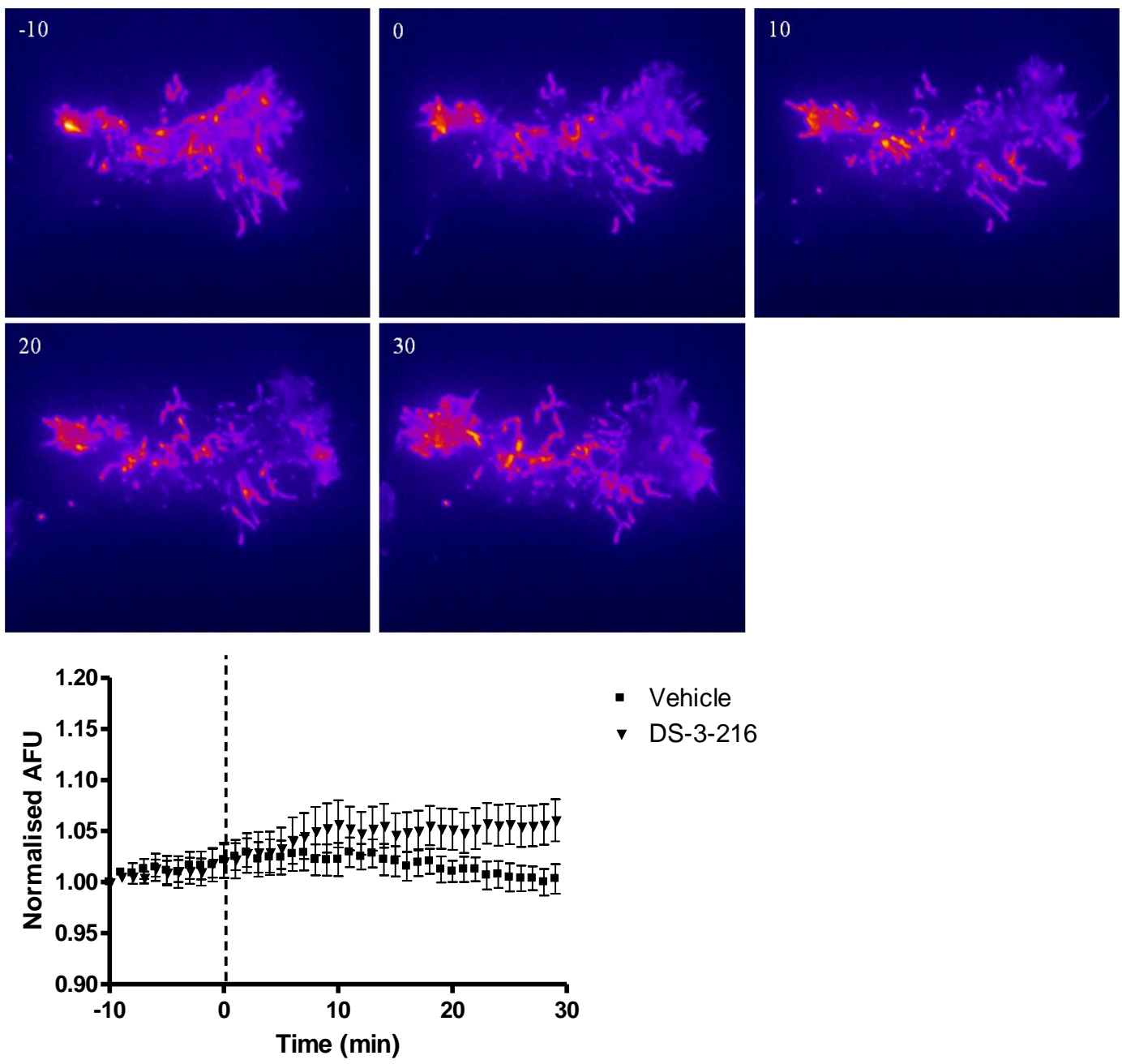

- Vehicle

DS-3-216
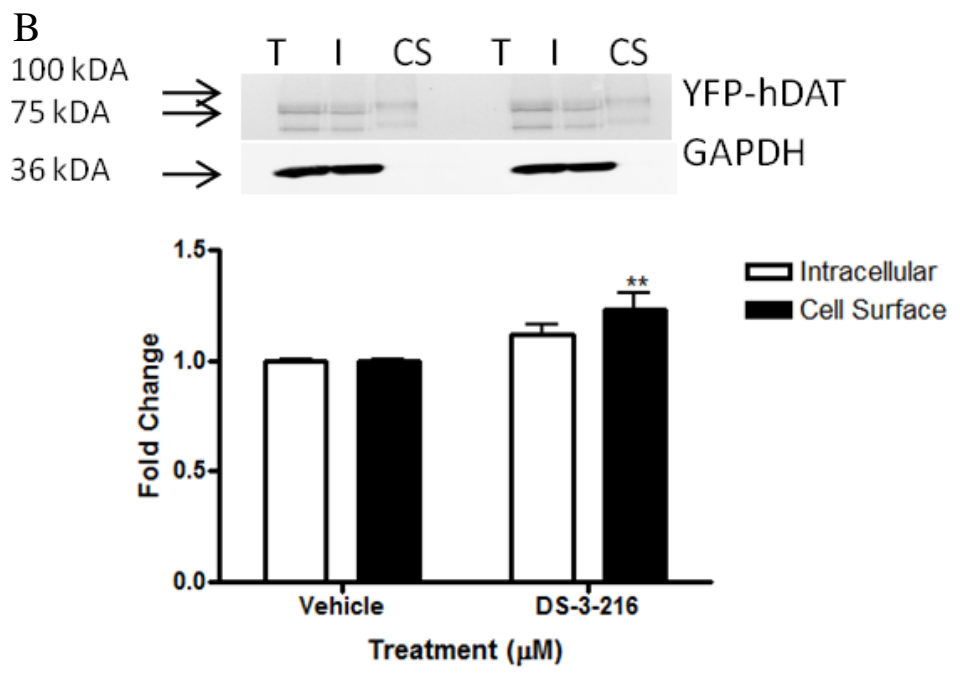

Figure 4.18: A 30 min incubation with DS-3-216 led to a significant increase in cell surface expression of YFP-hDAT. (A) TIRFM studies showed that after a $29 \mathrm{~min}$ incubation with DS-3-216, a non-significant increase in YFP-hDAT was seen, two-way ANOVA followed by Bonferroni post test $(n=12-19)$. A representative cell is shown with images at $-10,0,10,20$, and 30 min. (B) Cells were also incubated with DS-3-216 for $30 \mathrm{~min}$ before cell surface biotinylation experiments. A significant increase in cell surface expression of YFP-hDAT was seen, one-way ANOVA followed by Bonferroni post test $(\mathrm{n}=9) . \mathrm{T}=$ total, $\mathrm{I}=$ internal, $\mathrm{CS}=$ cell surface. 


\subsection{Discussion}

Previous studies have identified that acute KOPr activation increases DAT function in rat brain tissue and in cultured cells (Chapter 3, T. Shippenberg, unpublished data, Thompson et al. 2000). Here we showed that the classic KOPr agonists U50,488H and U69,593 and the novel agonists salvinorin A, DS-1-240, and DS-3-216 all caused acute increases in DAT function in cells transfected with YFP-hDAT and myc-rKOPr, and this increase in function was nor-BNI- and PTX-sensitive. Salvinorin A and U50,488H also caused increased DAT function after a 30 min incubation, with DS-1-240 and DS3-216 having no significant effects on $\mathrm{ASP}^{+}$uptake. One way in which increases in transporter function can occur is through increased cell surface expression. The RDEV results of Chapter 3 from rat brain tissue suggested that there was an increase in Vmax but not $\mathrm{Km}$ with the KOPr agonists, suggesting increased cell surface expression.. As the model used for those experiments was the low to infinite trans model, it was expected that these changes in cell surface expression either occurred immediately, or after a longer incubation since the compound of interest is present in the RDEV chamber for up to $1 \mathrm{~h}$. In HEK-293 cells co-transfected with YFP-hDAT and mycrKOPr, none of the compounds had an effect on cell surface expression of YFP-hDAT at the short time points studied, but there was an trend towards an increase in $\mathrm{ASP}^{+}$ binding to YFP-hDAT, which suggests there may be an increase in active YFP-hDAT at the cell surface. After a longer 30 min incubation, salvinorin A and U69,593 caused significant increases in YFP-hDAT expression at the cell surface in both TIRFM and cell surface biotinylation studies. DS-3-216 showed a significant increase in cell surface expression in biotinylation studies but a non-significant increase with TIRFM. The other compounds studied: U50,488H and DS-1-240, showed no change in cell surface expression at this later time point. These results are summarised in Table 4.2.

\subsubsection{Preliminary experiments}

Preliminary experiments were carried out to find the time of maximal effect of the agonists on DAT function. This was so that these time points could be used for subsequent experiments with confidence that an effect would not be missed. By taking slope measurements every $30 \mathrm{~s}$, it was found that salvinorin A had the fastest onset of action, followed by U50,488H and then DS-1-240 and DS-3-216 (Table 4.1). This may reflect the fast onset of action reported for salvinorin $\mathrm{A}$ in animals studies, where it is seen to reach the brain within $40 \mathrm{~s}$ after i.v. administration (Hooker et al. 2008). These data also mirror that found with RDEV in rat brain tissue (Chapter 3), in which the 
effect of salvinorin A was measured after 1 min; whereas, the other agonists' activities were seen at $4 \mathrm{~min}$.

Pilot experiments were also carried out to confirm the co-expression of YFP-hDAT and myc-rKOPr in all the cell lines used for this study (Figure 4.3). When cells are cotransfected, it is assumed that if one of the plasmids is expressed in a cell, the other will be as well. Figure 4.3 demonstrated that YFP-hDAT and myc-rKOPr were coexpressed in the same cells, with varying amounts of both plasmids seen in different cells. As there needs to be a ratio of about two KOPr to one DAT to see functional effects of KOPr activation (B. Kivell, personal communication), and as different cells express varying amounts of both plasmids, initial data was also used to find a suitable range of YFP-hDAT expression levels to collect data from. It was found in this study that cells that had as AFU value for YFP-hDAT between 250 and 1000 showed increases in $\mathrm{ASP}^{+}$uptake after KOPr agonist addition. This demonstrates that cells containing YFP-hDAT between this range contained the correct ratio of KOPr to DAT for modulation of DAT to occur. Data from cells with an AFU within this range were used for all future confocal microscopy experiments. It was also found that cells with an AFU for YFP-hDAT below 100 did not demonstrate linear uptake curves and; therefore, cells with an AFU for YFP below 100 were classed as non-transfected cells.

\subsubsection{Functional changes}

All of the KOPr agonists led to increased $\mathrm{ASP}^{+}$uptake at the acute time points between $60 \mathrm{~s}$ and $200 \mathrm{~s}$ (Figure 4.6, 4.8, 4.9). In HEK-293 cells, salvinorin A had the largest effect, with an increase of $40 \%$ at 1 min; whereas' the effects of the other agonists investigated were not maximal until $100 \mathrm{~s}(\mathrm{U} 50,488 \mathrm{H})$ or $200 \mathrm{~s}$ (DS-1-240 and DS-3216) after agonist addition. U50,488H, DS-1-240, and DS-3-216 had lesser effects on $\mathrm{ASP}^{+}$uptake by YFP-hDAT and caused increases of about $20 \%$ in HEK-293 cells. However, in the other cell types used to confirm these results, all compounds tested caused changes in uptake of between 10 and 25\%, with salvinorin A not showing an increased effect relative to the other compounds, as was seen in HEK-293 cells. This is most likely due to HEK-293 cells having differences in subtypes and amounts of signlling molecules compared to the other two cell lines. Overall, uptake in HEK-293 cells was higher than that seen in the other cell systems. Both N2A and COS7 cells are more difficult to transfect then HEK-293 cells, and even though more Lipofectamine 2000 reagent was used and double the plasmid DNA for each protein was used, 
transfection rates were generally lower than that of HEK-293 cells. Therefore from this data it was decided that all further experiments would be carried out in HEK-293 cells. Even though it would be best to carry out these experiments in a neuronally-derived cell line, such as N2A cells, there has been evidence that HEK-293 cells have neuronal properties (Shaw et al. 2002). HEK-293 cells stained for several proteins that are only expressed in neuronal cells, such as neurofilament subunit $\mathrm{M}$ and $\mathrm{L}$, and also stained for SERT which is not present in the kidney. Shaw et al. proposed that the transformation of HEK-293 cells with adenovirus 5 led to this neuronal protein expression, with adenovirus appearing to target neuronal cells rather than kidney cells. As there is a small population of cells with neuronal properties in the developing kidney the author suggests that these cells were targeted for transformation, leading to a kidney cell line which shows neuronal characteristics rather than displaying a strictly renal epithelial phenotype (Shaw et al. 2002).

The KOPr-1 is a $\mathrm{G}_{\mathrm{i} / \mathrm{o}}$ G protein-coupled receptor and is sensitive to PTX. PTX is a toxin produced by the bacterium Bordetella purtussis that causes whooping cough. PTX catalyses the ADP ribosylation of the $\alpha$ subunit of $\mathrm{G}$ proteins, preventing interaction with GPCR and preventing the transfer of GDP to GTP, keeping the Ga subunit in a GDP-bound state (Pizza et al. 1988). Incubation of HEK-293, N2A, and COS7 cells for 16-24 h with PTX before $\mathrm{ASP}^{+}$addition led to a complete inhibition of the effect of the KOPr agonists, confirming that these compounds work through a KOPr-1 pathway. A previous unpublished report also found that the effect of salvinorin A on DAT function was inhibited by PTX (T. Shippenberg, unpublished data). In HEK-293 cells, phosphorylation of ERK1/2 by the KOPr agonist U50,488H was inhibited by addition of PTX (200 ng/mL, $18 \mathrm{~h}$ before experiment) (Bruchas et al. 2007b), confirming our results in HEK-293 cells. Interestingly, it has been shown that DS-3-216 (MOM-salB) appears to have PTX insensitive effects in immortalised astrocytes in which incubation with $100 \mathrm{ng} / \mathrm{mL}$ of PTX for 16-24 h had no effect on DS-3-216-induced ERK1/2 phosphorylation (McLennan et al. 2008). Immortalised astrocytes treated with the classic KOPr agonist U69,593 in the presence of PTX showed no phosphorylation of ERK1/2, indicating that the phosphoryltion of ERK1/2 by DS-3-216 in the presence of PTX was specific to DS-3-216 (McLennan et al. 2008). The phosphorylation of MAPK by $\mathrm{U} 50,488 \mathrm{H}$ in $\mathrm{CHO}$ cells expressing hKOPr was also inhibited by prior incubation with PTX, but U50,488H internalisation was not inhibited by PTX, suggesting that activation of $\mathrm{G}$ proteins is not necessary for hKOPr internalisation ( $\mathrm{Li}$ et al. 1999). 
There have been no studies carried out on DS-1-240 with PTX, so the present study is the first to show that activation of YFP-hDAT by DS-1-240 is mediated through a $\mathrm{G}_{\mathrm{i} / \mathrm{o}}$ PTX sensitive mechanism. The data in this study show that the increase in $\mathrm{ASP}^{+}$uptake by YFP-hDAT is PTX-sensitive, suggesting that the modulation of DAT by the agonists investigated is carried out via a $\mathrm{G}_{\mathrm{i} / \mathrm{o}}$ dependent $\mathrm{KOPr}$ pathway.

Nor-BNI is a long-acting KOPr antagonist that has previously been shown to block the effects of U50,488H (Negus et al. 1997; Bruchas et al. 2006), salvinorin A (Chavkin et al. 2004; Braida et al. 2009), and DS-3-216 (McLennan et al. 2008; Wang et al. 2008) in animal and cell models. Our data also show that incubation with nor-BNI attenuates the effects of these agonists, along with DS-1-240, on YFP-hDAT function in all the cell lines studied. This confirms that the increase in $\mathrm{ASP}^{+}$uptake by YFP-hDAT seen by these agonists is KOPr mediated.

After a 30 min incubation with the KOPr agonist compounds, only U50,488H and salvinorin A caused a significant increase in $\mathrm{ASP}^{+}$uptake by YFP-hDAT (Figure 4.10). Both DS-3-216 and DS-1-240 have been shown to have longer half lives than that of salvinorin A (Harding et al. 2005; Wang et al. 2008) and are more potent at activating KOPr then salvinorin A (Harding et al. 2005; Wang et al. 2008). Therefore, it was expected that these compounds would lead to increased $\mathrm{ASP}^{+}$uptake by YFP-hDAT after $30 \mathrm{~min}$. One explanation could be that the more potent induction of cell surface internalisation of human KOPr by DS-3-216 compared to both salvinorin A and U50,488H (Wang et al. 2008) leads to a decrease in the interaction between KOPr and DAT. A study carried out in 2008 showed that while U50,488H, salvinorin A, and DS3-216 all induced a 40\% decrease in human KOPr cell surface expression after 30 min, DS-3-216 was much more potent, with $\mathrm{EC}_{50}$ values of $97.1 \mathrm{nM}, 522 \mathrm{nM}$, and $7.4 \mathrm{nM}$, respectively (Wang et al. 2008). DS-3-216 was also much more potent than U50,488H and salvinorin A at down-regulating KOPr by decreasing total mature KOPr, with DS3-216 showing an $\mathrm{EC}_{50}$ value of $6.3 \mathrm{nM}$, compared to $\mathrm{U} 50,488 \mathrm{H}$ and salvinorin $\mathrm{A}$ which gave values of $125 \mathrm{nM}$ and $142 \mathrm{nM}$, respectively (Wang et al. 2008). It has also been shown using co-immunoprecipitation and BRET that KOPr and DAT physically interact, and this is proposed to contribute to KOPr's ability to up-regulate DAT function (T. Shippenberg, unpublished data). This increase in potency for downregulation and internalisation may inhibit the interaction of KOPr and DAT, leading to 
no change in function seen at this time point for DS-3-216 and DS-1-240. Further studies will be needed to test this hypothesis.

\section{$\underline{4.5 .3 \text { Cell surface expression changes }}$}

As one of the ways in which DAT function is increased is by increasing cell surface expression, we investigated whether treatment with KOPr agonists could lead to increases in the cell surface expression of YFP-hDAT in HEK-293 cells. Activation of DRD2 and DRD3 receptors have both been shown to lead to increased DAT function and a rapid increase in cell surface expression of DAT (Bolan et al. 2007; Zapata et al. 2007). In the present study we found that while activation of KOPr led to a rapid increase in DAT function, there was no significant increase in YFP-hDAT cell surface expression during this time-frame with either TIRFM or cell surface biotinylation (Figure 4.12, 4.13). This data suggest that something other than cell surface expression is causing the increase in $\mathrm{ASP}^{+}$uptake seen with $\mathrm{KOPr}$ agonism at these rapid time points. As there was an increase in binding of $\mathrm{ASP}^{+}$to YFP-hDAT (Figure 4.11) it may be due to increased activation of previously inactive DAT already present on the cell surface. Another way in which transporters can be up-regulated is by increasing uptake turnover rates, which is another possibility for increased $\mathrm{ASP}^{+}$binding to YFP-hDAT (Meiergerd et al. 1994b).

To investigate if the increases in uptake of $\mathrm{ASP}^{+}$observed at $30 \mathrm{~min}$ with the $\mathrm{KOPr}$ agonists U50,488H and salvinorin A, were due to increased cell surface expression of DAT, cell surface biotinylation and TIRFM experiments were carried out for all the compounds investigated (Figure 4.14-4.18). Interestingly, there was no change in cell surface expression of YFP-hDAT seen with the classic agonist U50,488H but there was with the positive control, U69,593. This suggests that these two classic agonists may activate slightly different pathways and have different long-term effects. Previous studies have identified differences between the effects of U50,488H and U69,593 on KOPr mediated pathways. A study investigating the effects of chronic $(20 \mathrm{~h})$ incubation of $\mathrm{CHO}$ cells expressing hKOPr found that salvinorin $\mathrm{A}, \mathrm{U} 50,488 \mathrm{H}$, and $\mathrm{U} 69,593$ all decreased the expression of the $G$ protein subunit $G_{\alpha i 3}$; whereas, only $\mathrm{U} 50,488 \mathrm{H}$ increased expression of $\mathrm{G}_{12}$, providing evidence towards biased agonism that often occurs in tolerance to opioid agonists ( $\mathrm{Xu}$ et al. 2008). Furthermore, a study carried out to investigate the effects of KOPr on amphetamine-induced hyperlocomotion found that while both $\mathrm{U} 69,593$ and $\mathrm{U} 50,488 \mathrm{H}$ initially decreased the hyperlocomotion 
response, after $40 \mathrm{~min} \mathrm{U} 50,488 \mathrm{H}$ appeared to increase hyperlocomotion; whereas, U69,593 continued to attenuate it (Vanderschuren et al. 2000). The authors concluded that these different effects may be due to a KOPr-independent mechanism that is somehow activated by $\mathrm{U} 50,488 \mathrm{H}$.

The RDEV data of Chapter 3 suggested that there was an increase in cell surface expression of DAT after U50,488H and DS-1-240 treatment. Possible reasons why this may not have occurred in the cell studies of this chapter include the time point chosen to measure changes in cell surface expression, with a longer incubation (30-60 min) possibly leading to increased expression that wasn't seen in the cell studies. Also not all data from tissue models will translate to a cell model due to the differences between rat brain tissue samples and, for example, a kidney cell line like HEK-293. As HEK293 cells do not express the same proteins as NAc tissue, there may be many other interacting factors that are involved in the increased cell surface expression in tissue samples compared to isolated cells. For example dopaminergic neurons contain DA receptors that are not present in HEK-293 cells, and the signalling pathways within the rat brain tissue may be regulated differently to those found within HEK-293 cells.

After a 30 min incubation with salvinorin A, there was a significant increase in cell surface expression of YFP-hDAT, measured by both cell surface biotinylation and TIRFM. There were also significant increases seen with the novel compound DS-3-216 with cell surface biotinylation but not TIRFM. This was unexpected as $\mathrm{ASP}^{+}$uptake studies at this time point showed no changes in uptake, but these results do match the increases seen in the RDEV studies. No increase in cell surface expression was observed with DS-1-240 at $30 \mathrm{~min}$.

Both cell surface biotinylation and TIRFM experiments have limitations. As mentioned previously, cell surface biotinylation studies can overestimate the intracellular fraction due to biotin being unable to bind to proteins found within tight invaginations on the plasma membrane. Biotin can also have a low efficiency of binding to cell membrane proteins. TIRFM studies allow a small evanescent field to be visible from the cover slip. In our experiments we set our TIRFM system so that the evanescent field was close to $100 \mathrm{~nm}$ from the coverslip, which means that DAT contained in vesicles close to the plasma membrane would be measured as cell surface DAT. This means that when these vesicles fuse to the plasma membrane they are not measured as an increase 
in cell surface expression. However as vesicles of DAT further away from the cell membrane move towards and fuse with the membrane an increase in fluorescence is measured. TIRF experiments also do not allow measurement of the whole cell memebrane and therefore if the cell shape, adhesion, or motility occurs this may effect the measurements gained. Our results showed a larger increase in cell surface expression of YFP-hDAT with cell surface biotinylation compared to TIRFM. This difference between techniques has previously been observed (Furman et al. 2009a) and is thought to occur due to the abovementioned limitations of both techniques.

Overall, short-term exposure of HEK-293 cells to KOPr agonists did not alter cell surface expression of transfected YFP-hDAT, which suggests that the initial increase in YFP-hDAT function is modulated by a trafficking-independent process. After a $30 \mathrm{~min}$ incubation of the cells with the agonists, the addition of U69,593, salvinorin A, and DS3-216 led to increased cell surface expression of YFP-hDAT, with no changes observed for U50,488H and DS-1-240. 
Table 4.2: Summary of results of Chapter 4

\begin{tabular}{|l|l|l|l|l|l|}
\hline $\begin{array}{l}\text { KOPr } \\
\text { agonist }\end{array}$ & $\begin{array}{l}\text { Increased } \\
\text { ASP } \\
\text { uptake } \\
\text { acute } \\
(<5 \text { min) }\end{array}$ & $\begin{array}{l}\text { Increased } \\
\text { ASP } \\
\text { uptake } \\
\mathbf{3 0} \text { min }\end{array}$ & $\begin{array}{l}\text { Increased cell } \\
\text { surface } \\
\text { expression of } \\
\text { YFP-hDAT, } \\
\text { acute } \\
(<\mathbf{5} \text { min })\end{array}$ & $\begin{array}{l}\text { Increased } \\
\text { cell surface } \\
\text { expression } \\
\text { of YFP- } \\
\text { hDAT at 30 } \\
\text { min }\end{array}$ & $\begin{array}{l}\text { Increased } \\
\text { binding of ASP } \\
\text { acute } \\
(<5 \text { min })\end{array}$ \\
\hline U50,488H & Yes & Yes & No & No & Not significant \\
\hline U69,593 & $\begin{array}{l}\text { Not } \\
\text { investigated }\end{array}$ & $\begin{array}{l}\text { Not } \\
\text { investigated }\end{array}$ & No & Yes & $\begin{array}{l}\text { Not } \\
\text { investigated }\end{array}$ \\
\hline Salvinorin & Yes & Yes & No & Yes & Not significant \\
\hline DS-1-240 & Yes & No & No & No & Yes \\
\hline DS-3-216 & Yes & No & No & Yes & Not significant \\
\hline
\end{tabular}




\subsection{Conclusion}

To conclude, all of the KOPr agonists investigated led to increased YFP-hDAT function, as measured by $\mathrm{ASP}^{+}$uptake, at acute time points. This increase in function was not caused by an increase in cell surface expression at these time points as measured by cell surface biotinylation and TIRFM. However, there was a nonsignificant increase in $\mathrm{ASP}^{+}$binding observed which suggests that the increase in uptake may be due to increased active DAT at the cell surface. At a $30 \mathrm{~min}$ time point salvinorin $\mathrm{A}$ and $\mathrm{U} 50,488 \mathrm{H}$ still caused a significant increase in $\mathrm{ASP}^{+}$uptake. Cell surface expression studies at this time point showed that salvinorin A, U69,593, and DS-3-216 led to increased cell surface expression of YFP-hDAT, whereas no change was observed with U50,488H or DS-1-240. This implies that the KOPr agonists studied may have different effects on DAT. This study demonstrated that the modulation of DAT by KOPr can be measured successfully in a cell model, and that further investigation into the effects of these agonists on DAT function is warranted. 


\section{Chapter 5: Involvement of the ERK1/2 signalling pathway and lipid rafts on the modulation of DAT by KOPr}

\subsection{Introduction}

Cell membranes are thought to contain sphingolipid- and cholesterol-dense areas called lipid rafts. Lipid rafts are localised areas of the plasma membrane in which cell membrane proteins can be more easily regulated, and these areas of unique lipid composition are thought to be involved in processes such as endocytosis and cell signalling (Kenworthy et al. 2004). Although many studies support the existence of these regions, there is still contention as to their existence and the role in which they play in the cell (Reviewed by Pike 2003). It is also not known exactly how these rafts are structured. There are several different hypotheses put forward on the types of lipid raft that are found in cells, including stable immobile rafts, stable mobile rafts, dynamic partitioning in which molecules move in and out of, and no rafts at all (Kenworthy et al. 2004).

Both KOPr and DAT have been found within lipid-rich regions of the cell membrane, as well as throughout the rest of the cell membrane (Xu et al. 2006; Adkins et al. 2007; Foster et al. 2008). Regulation of DAT by the PKC activator PMA differs between lipid rafts and non-lipid rafts within the cell membrane, suggesting that DAT found in lipid rafts may have a different regulatory role then DAT distributed within the rest of the cell membrane (Foster et al. 2008). Interestingly, lipid raft disruption by the cholesterol depleter, methyl- $\beta$-cyclodextrin (M $\beta C$ ), does not lead to movement of DAT from lipid rafts into the surrounding membrane, which is what occurs with the NET and SERT associated within lipid rafts (Foster et al. 2008), but cholesterol depletion did lead to decreased function of DAT. One report demonstrated that this effect was not due to changes in DAT cell surface expression (Adkins et al. 2007), but another found that DAT expression decreased by $16 \%$ in the cell membrane (Foster et al. 2008). These differences may be due to the different cell lines used, with the first study using murine N2A cells (Adkins et al. 2007) and the other pig renal epithelial cells (LLCPK $)$ (Foster et al. 2008). Overall these data suggest that lipid rafts are involved in the functional regulation of DAT and that disruption of lipid rafts leads to a decrease in function, possibly as a result of a decrease in cell surface expression. 
One study has reported that KOPr is localised within lipid rafts (Xu et al. 2006). In CHO cells expressing FLAG-hKOPr, disruption of lipid rafts with $\mathrm{M} \beta \mathrm{C}$ led to an increase in affinity of hKOPr for $\mathrm{U} 50,488 \mathrm{H}$, an increase in interaction between $\mathrm{hKOPr}$ and $\mathrm{G}_{\mathrm{i} / \mathrm{o}}$, and an increase in ERK1/2 activation (Xu et al. 2006). It was suggested that lipid rafts are involved in the coupling of GPCR to G proteins and may be involved in the conformation of the KOPr receptor. Overall these data show that the lipid raft environment is important for the functional regulation of both KOPr and DAT, and the disruption of this environment leads to dysregulation of the signalling systems that activate and regulate these two transport proteins.

A signalling pathway that both KOPr and DAT share is the ERK1/2 pathway. KOPr activation leads to activation of ERK1/2 (Belcheva et al. 2005; Kim et al. 2006; McLennan et al. 2008), and activation of ERK1/2 leads to increased DAT function and cell surface expression (Moron et al. 2003; Bolan et al. 2007; Zapata et al. 2007). The ERK1/2 pathway is also involved in the action of drugs of abuse, with acute cocaine and amphetamine causing a rapid and transient increase in ERK1/2 phosphorylation that is mediated through DRD1 receptors and NMDA receptors (Valjent et al. 2000; Valjent et al. 2005). Thus, the link between novel KOPr agonists and the ERK1/2 pathway may be significant in identifying potential pharmacotherapies for drug abuse. 


\subsection{Aims}

The aim of this chapter is to investigate possible ERK1/2-related mechanisms in which KOPr activation leads to increased DAT function and the role of lipid raft localisation in this process. Both KOPr and DAT are found within lipid rafts and both have links to ERK1/2 signalling. One relationship to be tested is whether the increase in cell surface expression seen with both U69,593 and salvinorin A is affected by ERK1/2 inhibition. It is hypothesised that lipid raft disruption and inhibition of ERK1/2 will prevent the increases in DAT function caused by KOPr activation, and that ERK1/2 inhibition will also inhibit the increased cell surface expression of DAT after a 30 min incubation with these drugs (see Chapter 4). 


\subsection{Methods}

\subsubsection{Western blotting for total protein}

HEK-293 cells were plated and transfected as previously described (sections 2.1.2 and 2.1.3). To investigate if the increase in cell surface expression seen with U69,593 and salvinorin A is due to changes in total protein, YFP-hDAT-transfected HEK-293 cells were treated with $10 \mu \mathrm{M}$ of each KOPr agonist for $30 \mathrm{~min}$. The cells were lysed for 1 hr at $4^{\circ} \mathrm{C}$ on a rocker in $400 \mu \mathrm{L}$ of RIPA buffer containing $0.01 \%$ protease inhibitor. Cell lysates were transferred into microcentrifuge tubes and centrifuged in a benchtop microcentrifuge for $30 \mathrm{~min}$ at $4^{\circ} \mathrm{C}$ at $16,000 \mathrm{~g}$. A $50 \mu \mathrm{L}$ sample was removed from each lysate and $15 \mu \mathrm{L}$ of 5 x reducing buffer containing $10 \% \beta$-mercaptoethanol was added (Appendix 1) and $20 \mu \mathrm{L}$ loaded onto a 10\% SDS-PAGE gel (Appendix 1). The gel was run for $90 \mathrm{~min}$ at $120 \mathrm{~V}$, and the fluorescence of YFP-hDAT was read on a fluorescence laser scanner ((Fujifilm FLA-5100, Fuji Film). The proteins on the gel were then transferred onto a PVDF membrane as described in section 2.4. Tubulin was probed as a loading control, YFP-hDAT was normalised to tubulin and then normalised to controls on each gel to allow comparisons between gels.

\subsubsection{Lipid raft disruption}

HEK-293 cells were plated and transfected as previously described (sections 2.1.2 and 2.1.3), and $\mathrm{ASP}^{+}$uptake experiments were carried out (Section 2.5.1). To disrupt lipid rafts, cells were incubated for $10 \mathrm{~min}$ with $10 \mathrm{mM} \mathrm{M \beta C} \mathrm{(Sigma)} \mathrm{prior} \mathrm{to} \mathrm{the} \mathrm{ASP}^{+}$ uptake measurements. Data were analysed as previously described (Section 2.5.3). To investigate the effect of lipid raft disruption on basal $\mathrm{ASP}^{+}$uptake of transfected and non-transfected cells, the uptake before addition of the compound was calculated and then normalised to the YFP-hDAT expression for each cell. Cells were classed as nontransfected if their YFP-hDAT expression was under 100 AFU. Only cells with a YFPhDAT expression of between 250 and 1000 AFU were used in the study, as described in Chapter 4.

\subsubsection{Fluorescence recovery after photo-bleaching (FRAP)}

\subsubsection{Experimental protocol}

HEK-293 cells were plated and transfected in $35 \mathrm{~mm}$ glass-bottom dishes as previously described (section 2.1.2 and 2.1.3). FRAP was conducted as previously described (X. $\mathrm{Yu}$, personal communication, Adkins et al. 2007). An Olympus Fluoview FV1000 confocal microscope was used to conduct FRAP. The culture medium was replaced 
with $1 \mathrm{~mL}$ of $37^{\circ} \mathrm{C} \mathrm{KREBS}$ buffer, and the cells were placed in a heated cell chamber on the microscope stage and left to equilibrate for $5 \mathrm{~min}$. Using the $40 \mathrm{X}$ water objective lens, a single cell with a distinct plasma membrane was brought into focus and zoomed in on. Using the time controller, a protocol was created so that 4 pre-bleach images were taken $10 \mathrm{~s}$ apart using the 473 laser with the YFP filter (485 nm excitation; $545 \mathrm{~nm}$ emission). The pre-bleach imaging was followed by bleaching of a ROI of the plasma membrane of the cell. ROIs were elliptic in shape with an average diameter of $4 \mu \mathrm{m}$. Bleaching was conducted over 200 images taken at maximum speed with the 473 laser set at $100 \%$. After bleaching, post-bleaching images were collected, with 27 images taken $10 \mathrm{~s}$ apart. This allowed enough time for maximum recovery of the bleached region. For $\mathrm{M} \beta \mathrm{C}$-treated samples, cells were treated with $10 \mathrm{mM} \mathrm{M} \beta \mathrm{C}$ for $10 \mathrm{~min}$ before replacement of the culture medium with KREBS buffer.

\subsubsection{Data analysis}

To analyse the FRAP data, the pre- and post-bleach images were appended using Fluoview FV1000 software. Then three ROIs were taken using the Fluoview FV1000 software. These three ROIs were: a) the bleached region, b) the whole cell (a control for estimating unintentional photo-bleaching), and c) a non-fluorescent background control. The integrated fluorescence was determined at different time points for these three regions. The bleached region was then corrected for background and unintentional photo-bleaching and normalised so that the pre-bleach fluorescence intensity was 1 . The normalised data were then entered into GraphPad Prism, and the FRAP parameters Mf (mobile fraction, or recovery plateau) and $t_{1 / 2}$ (time taken for half Mf to be reached) were found using a one-phase exponential association non-linear regression model.

\subsubsection{Western blotting for P-ERK1/2}

HEK-293 cells were plated and transfected in $35 \mathrm{~mm}$ plastic petri dishes as previously described (sections 2.1.2 and 2.1.3).

For time course experiments, cells were serum-starved for 30 min in DMEM followed by UV treatment for $1 \mathrm{~h}$ (positive control), or treatment with KOPr agonist for $0 \mathrm{~s}, 60 \mathrm{~s}$, $100 \mathrm{~s}$ (U50,488H only), $200 \mathrm{~s}$ (DS-1-240 and DS-3-216 only), 5, 10, 20, 30, and 60 min. For inhibitor experiments, cells were serum-starved for $30 \mathrm{~min}$ and then treated with the MEK inhibitor 1,4-diamino-2,3-dicyano-1,4-bis $(o$ - 
aminophenylmercapto)butadiene (U0126, $20 \mu \mathrm{M}$, Sigma) for $30 \mathrm{~min}$ before KOPr agonist addition. MEK directly phosphorylates ERK1/2; therefore, the inhibition of MEK prevents the activation of ERK1/2. KOPr agonist was added for the time that caused the largest activation of P-ERK1/2 in the time course experiments (salvinorin A $=60 \mathrm{~s}$ and U50,488H, DS-1-240, and DS-3-216 = $5 \mathrm{~min}$ ). Cells were then washed $2 \mathrm{x}$ with sterile PBS and lysed at $4{ }^{\circ} \mathrm{C}$ with gentle shaking in $200 \mu \mathrm{L}$ of RIPA buffer containing $0.01 \%$ protease inhibitor (Sigma) and $0.01 \%$ phosphatase inhibitor (Sigma). Cell lysates were then transferred into microcentrifuge tubes and centrifuged at $16,000 \mathrm{~g}$ for $30 \mathrm{~min}$ at $4^{\circ} \mathrm{C}$. A $50 \mu \mathrm{L}$ sample was removed and mixed with $15 \mu \mathrm{L}$ of running buffer (Appendix 1), and $20 \mu \mathrm{L}$ of sample was loaded onto a 10\% SDS-PAGE gel (Appendix 1). Gels were run at $120 \mathrm{~V}$ for $90 \mathrm{~min}$, then scanned directly for YFP-hDAT on a Fuji-Film gel scanner. After scanning, the proteins were transferred to PVDF membrane, and Western blotting was conducted as described in section 2.4. P-ERK1/2 (1:500, overnight, Santa Cruz) was immunoblotted first and detected using anti-mouse Cy5 (1:2,500, $1 \mathrm{~h}$, GE Healthcare). Membranes were then stripped for $40 \mathrm{~min}$ in stripping buffer (Appendix 1) then washed $3 \mathrm{x}$ in T-TBS before reblocking. ERK1/2 (1:1,000, overnight, Cell Signalling) was then immunoblotted and detected using antirabbit Cy 3 (1;2,500, 1 h, GE Healthcare).

To analyse data, band intensities were measured using Image J, and P-ERK1/2 was normalised to total ERK1/2 expression.

\subsection{5 $\mathrm{ASP}^{+}$uptake for P-ERK1/2 inhibition}

Acute $\mathrm{ASP}^{+}$experiments and data analysis were carried out as previously described (Section 2.5) except cells were incubated with $20 \mu \mathrm{M}$ U0126 for 30 min before ASP $^{+}$ addition to inhibit MEK/ERK signalling.

\subsubsection{Biotinylation and TIRFM for P-ERK1/2 inhibition}

Cell surface biotinylation and TIRFM experiments were carried out as previously described (Section 2.6 and 2.3, respectively), except that cells were incubated with 20 $\mu \mathrm{M}$ of $\mathrm{U} 0126$ for $30 \mathrm{~min}$ before KOPr agonist addition. 


\subsection{Results}

\subsubsection{Total YFP-hDAT protein}

To investigate if the increases in cell surface expression induced by U69,593, salvinorin A, and DS-3-216 were due to increases in total YFP-hDAT protein in the cells, Western blotting was performed on cell lysates after incubation of the cell for $30 \mathrm{~min}$ with U69,593, U50,488H, salvinorin A, DS-1-240, and DS-3-216. Protein lysates were run on SDS-PAGE gels and scanned to visualise YFP-hDAT fluorescence on the gel and then Western transferred and immunoblotted for anti-tubulin as a loading control. Results showed that there was no significant increase in total YFP-hDAT protein in the cell after a 30 min incubation with any of the compounds investigated (Figure 5.1). This indicates that the increases in cell surface expression caused by U69,593, salvinorin A and DS-3-216 were not due to an overall increase in YFP-hDAT protein but due to increased exocytosis of YFP-hDAT to the plasma membrane. 


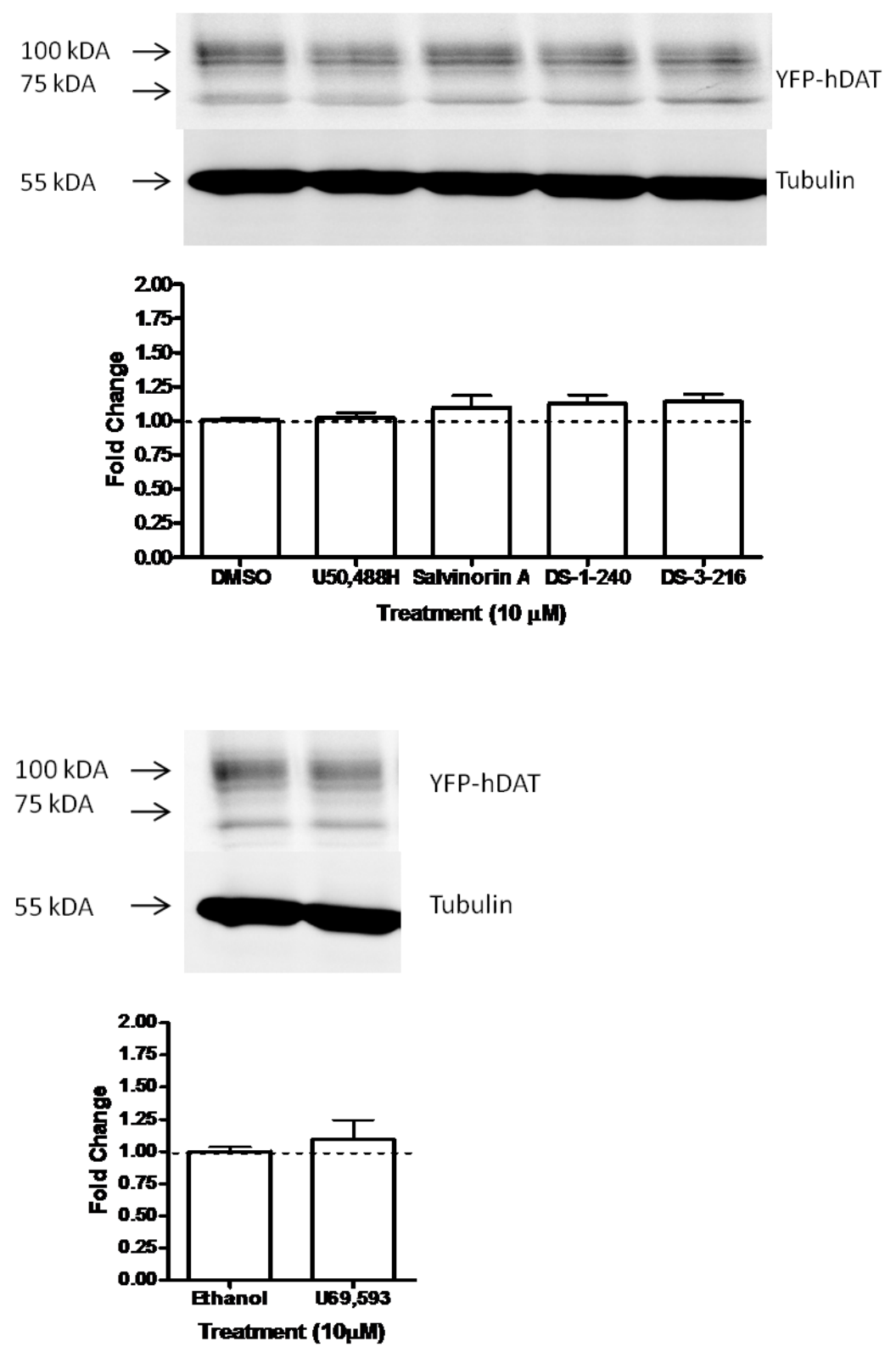

Figure 5.1: Treatment for 30 min with KOPr agonists has no effect on total YFPhDAT protein in HEK-293 cells. HEK-293 cells were treated with U50,488H, salvinorin A, DS-1-240, DS-3-216, U69,593, or their vehicles for $30 \mathrm{~min}$ before lysing and analysing for total YFP-hDAT protein and tubulin. Shown are a fluorescent scan of representative gel and the corresponding anti-tubulin Western blot. There was no significant change in total YFP-hDAT protein with any of the treatments $(n=5-6$ preparations). One-way ANOVA followed by Bonferroni post test. 


\subsubsection{Lipid raft disruption}

KOPr and DAT are found within lipid rafts (Xu et al. 2006; Adkins et al. 2007), and both proteins co-localise in transfected cells (T. Shippenberg, unpublished data). Therefore, disruption of lipid rafts would be expected to attenuate the effects of KOPr agonists on DAT function. $\mathrm{M} \beta \mathrm{C}$ disrupts lipid rafts by desorbing cholesterol from the cell membrane (Lawrence et al. 2003). Treatment of non-transfected and transfected HEK-293 cells with $10 \mathrm{mM} \mathrm{M} \beta \mathrm{C}$ for $10 \mathrm{~min}$ led to significant increases in uptake of $\mathrm{ASP}^{+}$of $100 \%$ (from $126 \pm 17$ to $247 \pm 40 \mathrm{AFU}$ ) and $50 \%$ (from $556 \pm 23$ to $777 \pm 42$ AFU), respectively (Figure 5.2). These increases in $\mathrm{ASP}^{+}$uptake are most likely due to the disruption of lipid rafts increasing cell permeability, leading to an increase in passive $\mathrm{ASP}^{+}$movement into the cell. The non-specific uptake (non-transfected cells) was then removed from transfected cell uptakes from each of the groups to investigate the specific effects of lipid raft disruption on YFP-hDAT function in these cells (Table 5.1). Interestingly, there was a significant increase of $23 \%$ in $\mathrm{ASP}^{+}$uptake in $\mathrm{M} \beta \mathrm{C}$ treated cells after the non-specific uptake was removed, suggesting that rather than decreasing YFP-hDAT function, lipid raft disruption increased YFP-hDAT transport function in these cells.

To confirm that M $\beta C$ was disrupting lipid rafts, FRAP was also carried out. FRAP is a technique in which a region of a living cell membrane expressing fluorescent membrane proteins is bleached and the recovery of fluorescence is measured. As the cell membrane is a fluid environment, FRAP measures the diffusion of the fluorophore through the cell membrane and also measures any exocytosis (insertion) of the fluorophore that occurs (Figure 5.3a). For this experiment an average recovery of $74.3 \%$ was seen for control experiments and $74.6 \%$ for $\mathrm{M} \beta \mathrm{C}$-treated samples (Figure 5.3b). FRAP ROIs were used that were large enough to include both lipid raft and nonlipid raft regions of the cell membrane. Lipid rafts have been shown to be between 10 and $200 \mathrm{~nm}$ in diameter (Adkins et al. 2007), and an average ROI of $4 \mu \mathrm{m}$ was used, which was consistent between M $\beta C$-treated (average ROI of $3.975 \mu \mathrm{m}$ ) and non-treated groups (average ROI of $4.095 \mu \mathrm{m}$ ) (Figure 5.3c). Treatment of cells with $\mathrm{M} \beta \mathrm{C}$ led to a significant increase in $t_{1 / 2}$ of $57 \%$ and no change to Mf, suggesting that lipid raft disruption led to a decreased mobility of YFP-hDAT, changing cell membrane dynamics (Figure 5.3d and e). 
To measure the effect of lipid raft disruption on KOPr activation of YFP-hDAT function, cells were treated with $10 \mathrm{mM} \mathrm{M \beta C} 10 \mathrm{~min}$ before $\mathrm{ASP}^{+}$uptake studies were conducted. The treatment of cells with $\mathrm{M} \beta \mathrm{C}$ led to a significant attenuation of the increase in DAT function caused by $10 \mu \mathrm{M}$ KOPr agonist with all agonists used (Fig 5.4a-d). As these $\mathrm{ASP}^{+}$uptake experiments measure a change in function due to KOPr agonist treatment within the same cell, it can be inferred that the inhibition seen was due to interference with the KOPr/DAT interaction rather than a direct disruption of DAT function, since the cells were still able to take up $\mathrm{ASP}^{+}$while in the presence of $\mathrm{M} \beta \mathrm{C}$. 
A

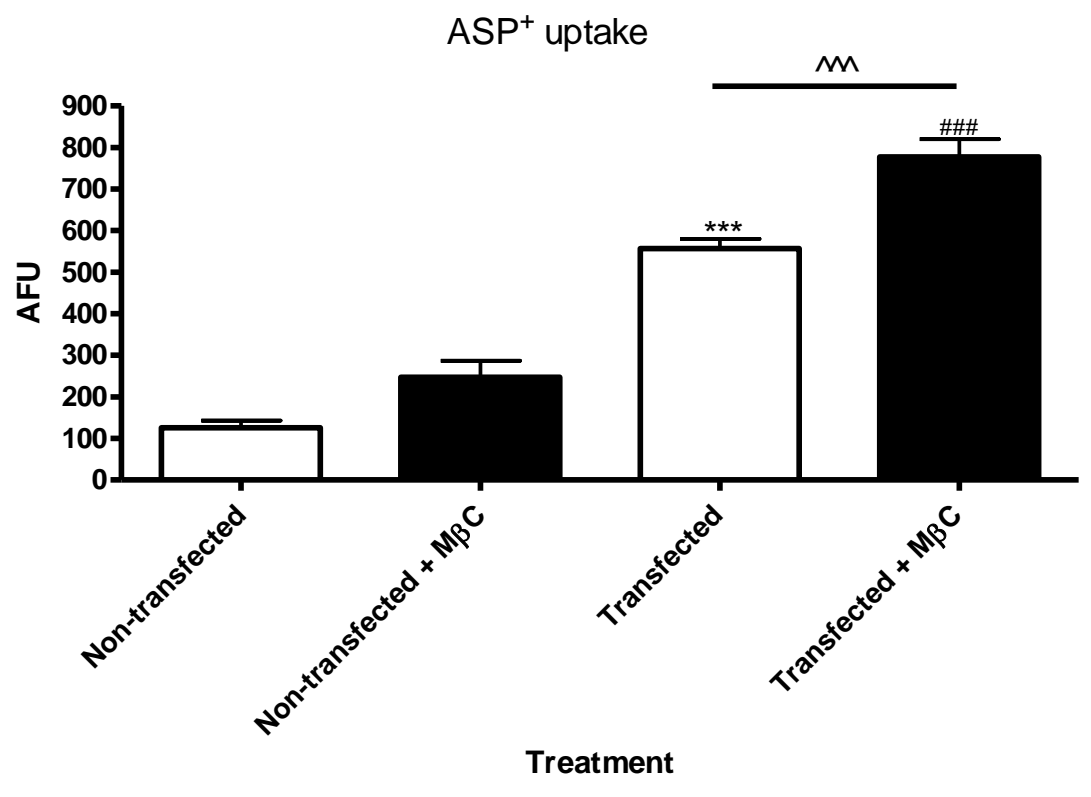

B

YFP-hDAT expression

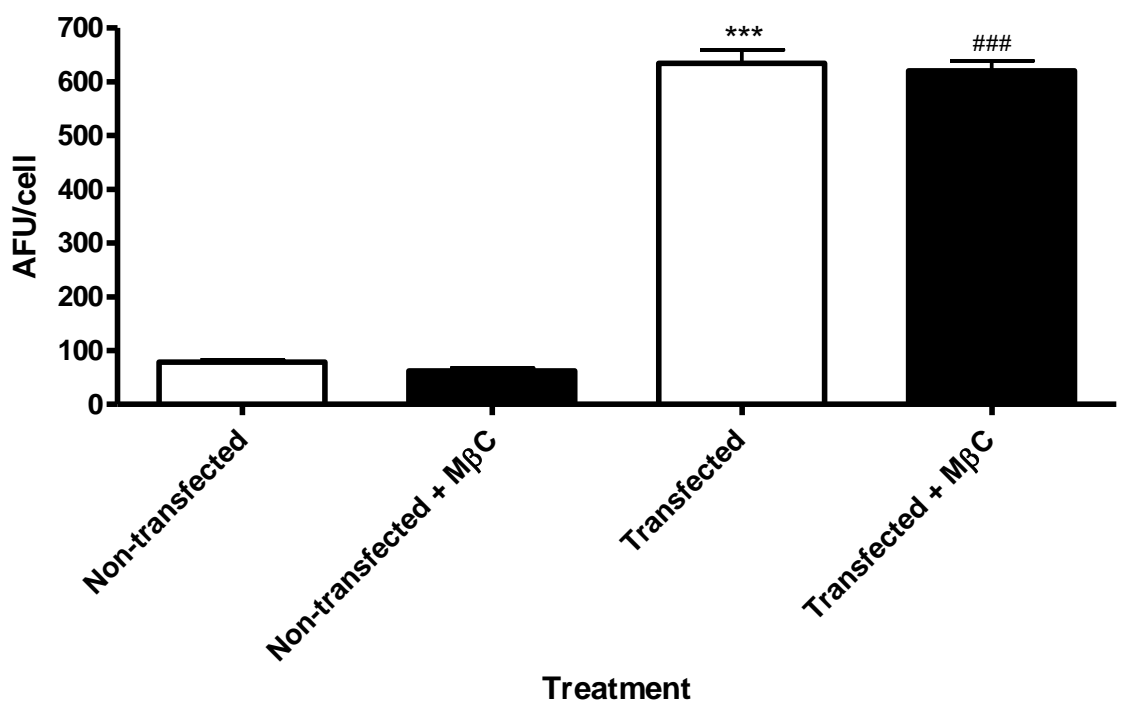

Figure 5.2: Effect of $\mathrm{M \beta C}$ in non-transfected and transfected cells on $\mathrm{ASP}^{+}$ uptake. (A) There were significant differences between $\mathrm{ASP}^{+}$uptake in nontransfected vs transfected cells for each treatment group. There was a non-signficant increase in $\mathrm{ASP}^{+}$uptake in non-transfected $+\mathrm{M} \beta \mathrm{C}$ cells compared to non-transfected cells without $\mathrm{M} \beta \mathrm{C}(\mathrm{p}=0.0652)$. Uptake by transfected cells was signficantly different between the $\mathrm{M} \beta \mathrm{C}$-treated and control groups (One-way ANOVA). (B) YFP-hDAT expression was consistent between groups, with transfected groups having a significantly higher YFP-hDAT epxression then non-transfected controls $(n=20-27$ cells, over 3 separate transfections, one-way ANOVA followed by Bonferroni post test. $* * * p<0.001$ compared to non-transfected control, \#\#\#p<0.001 compared to nontransfected $+\mathrm{M} \beta \mathrm{C}$ control, ${ }^{\wedge \wedge} \mathrm{p}<0.001$ compared to transfected control. 
Table 5.1: The effect of MBC treatment on $\mathrm{ASP}^{+}$uptake

\begin{tabular}{|l|l|l|}
\hline & Control cells & MBC treated cells \\
\hline Non-transfected & $126.0 \pm 16.93$ & $247.3 \pm 39.87^{*}$ \\
\hline Transfected & $556.5 \pm 23.48$ & $777.6 \pm 42.28^{* * *}$ \\
\hline Transfected - non-transfected & $430.5 \pm 23.07$ & $530.3 \pm 42.81^{*}$ \\
\hline
\end{tabular}

Mean \pm SEM, Student's t-test between control and M $\beta C$-treated cells, $* \mathrm{p}<0.05$, $* * * \mathrm{p}<0.001$. 

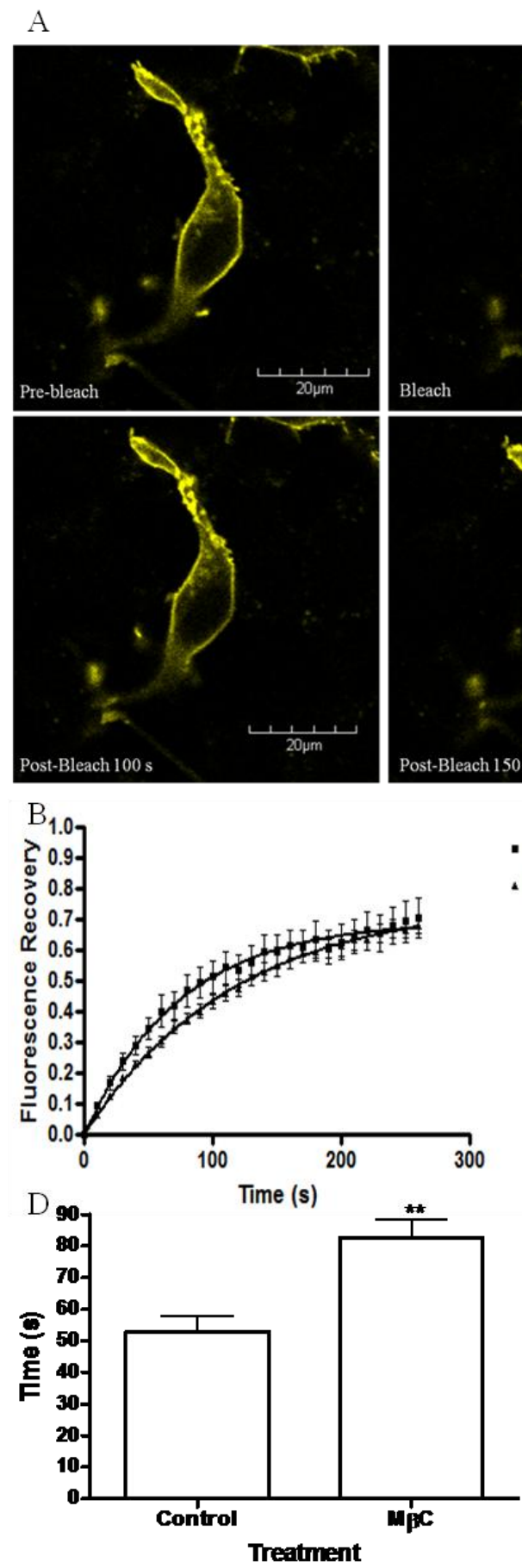
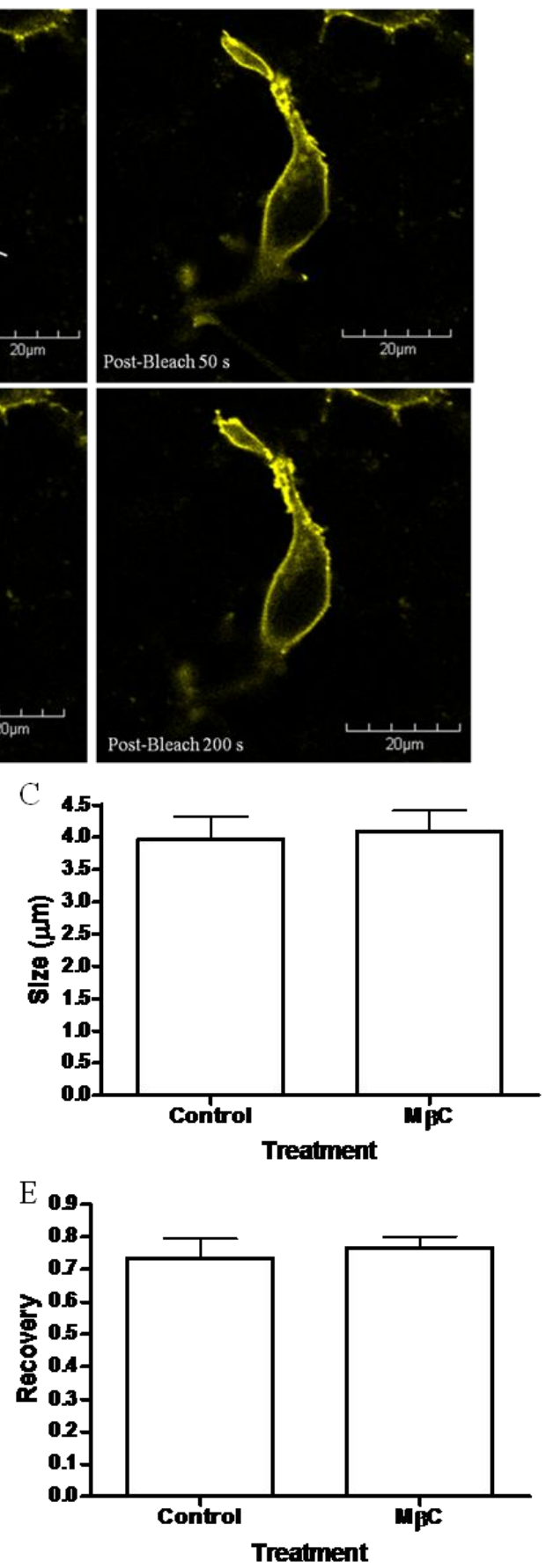

Figure 5.3: FRAP in HEK-293 cells expressing YFP-hDAT and myc-rKOPr with and without $10 \mathrm{mM} \mathrm{M \beta C.} \mathrm{(A)} \mathrm{Time} \mathrm{course} \mathrm{of} \mathrm{a} \mathrm{representative} \mathrm{FRAP} \mathrm{experiment}$ showing a pre-bleach image, a bleach image and post-bleach images at 50, 100, 150, and $200 \mathrm{~s}$ (For representative movie see DVD). (B) The average fluorescence recovery vs. time after bleaching in the FRAP experiments conducted. Recovery after $260 \mathrm{~s}$ was $74.3 \%$ of initial fluorescence for control samples, and $74.6 \%$ for $\mathrm{M} \beta \mathrm{C}$-treated samples. (C) The sizes of the ROI of bleach regions between control and $\mathrm{M} \beta \mathrm{C}$ treatment were the same, with averages of $3.975 \mu \mathrm{M}$ and $4.095 \mu \mathrm{M}$, respectively. (D) The average $\mathrm{t}_{1 / 2}$ of recovery of control vs. $\mathrm{M} \beta \mathrm{C}$-treated cells. $\mathrm{M} \beta \mathrm{C}$ treatment led to a significant increase in recovery $t_{1 / 2}$. (E) The average Mf of recovery was not different between control and M $\beta C$-treated groups ( $n=5-6$ cells, ${ }^{* *} \mathrm{p}<0.01$, Student's t-test). 

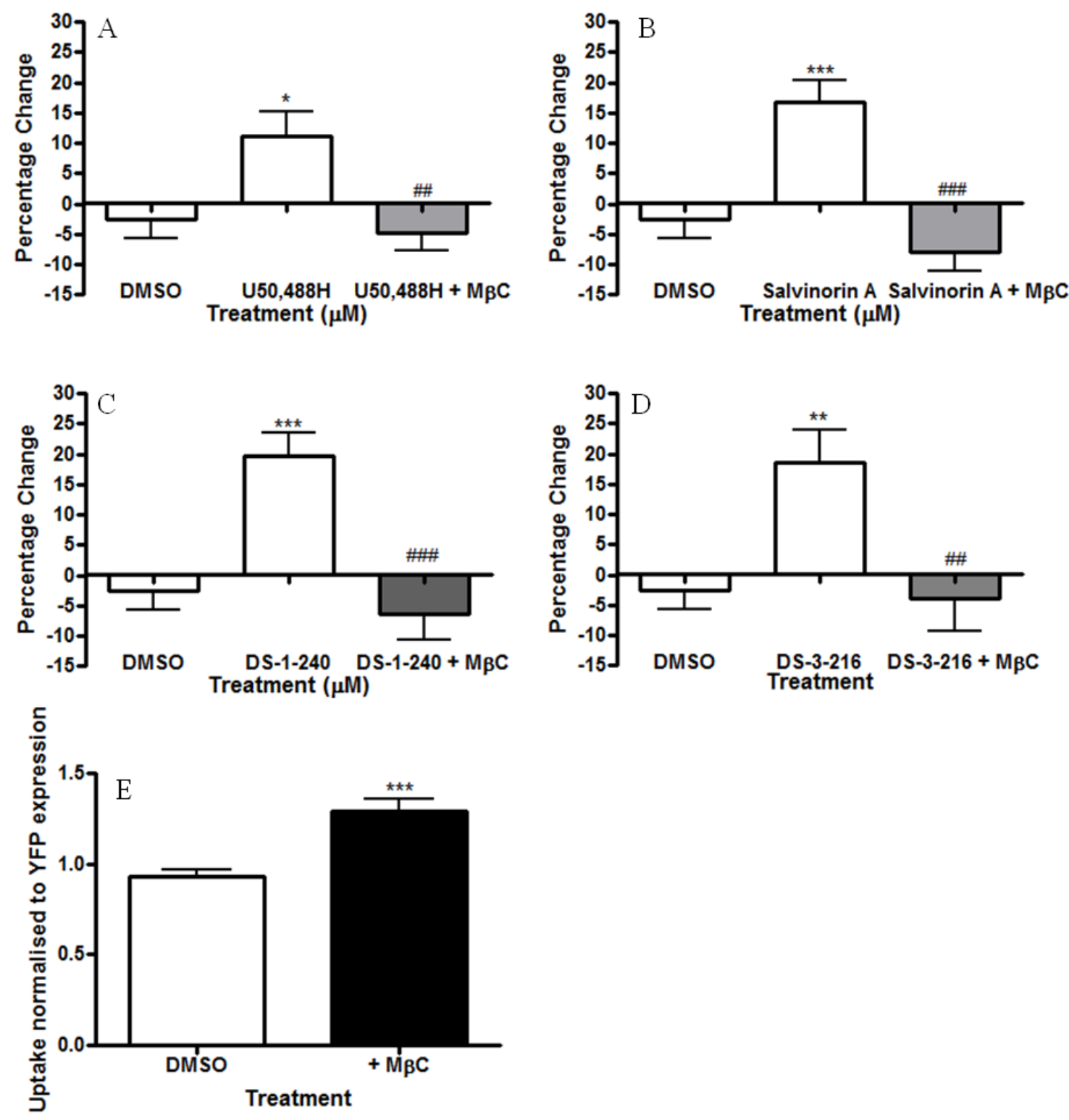

Figure 5.4: Lipid raft disruption attenuates the KOPr agonist-induced increase in DAT function. HEK-293 cells expressing YFP-hDAT and myc-rKOPr were incubated with $\mathrm{M} \beta \mathrm{C}(10 \mathrm{mM})$ for $10 \mathrm{~min}$ before $\mathrm{ASP}^{+}$uptake studies were conducted. Results showed that the addition of $\mathrm{M} \beta \mathrm{C}$ significantly blocked the effect of $10 \mu \mathrm{M}$ (A) U50,488H, (B) salvinorin A, (C) DS-1-240, and (D) DS-3-216 at their maximal time of function (100, 60, 200, $200 \mathrm{~s}$ respectively). Addition of DMSO as a vehicle control did not lead to a significant decrease in uptake compared to 0 for any of the compounds tested (One-sample Student's t-test; $\mathrm{n}=38-54$ cells). (E) $\mathrm{M} \beta \mathrm{C}$ led to a significant increase in $\mathrm{ASP}^{+}$uptake on its own (Student's t test, $* * * \mathrm{p}<0.001$ ). One-way ANOVA followed by Bonferroni post test, $* \mathrm{p}<0.05, * * \mathrm{p}<0.01$, ***p $<0.001$ compared to the DMSO control, \#\#p<0.05, \#\#\#p<0.001 compared to $10 \mu \mathrm{M}$ KOPr agonist. 


\subsubsection{ERK1/2 activation}

Previous studies have shown that KOPr agonists activate ERK1/2 (Belcheva et al. 2005; Kim et al. 2006; McLennan et al. 2008), and ERK1/2 activation leads to increased DAT function (Zapata and Shippenberg 2002; Moron et al. 2003; Bolan et al. 2007). A novel MOPr agonist herkinorin, which is based on the structure of Sal A, has also been shown to cause ERK1/2 activation (Groer et al. 2007), as has the novel KOPr agonist DS-3216 (McLennan et al. 2008). As ERK1/2 activation increases DAT function, the ERK1/2 pathway is a possible link between KOPr and DAT. Therefore, the effect of the KOPr agonists in this study on ERK1/2 activation was investigated, as well as the effect of ERK1/2 inhibition on DAT function. Initial time course studies were conducted to find the time where ERK1/2 phosphorylation peaked after KOPr agonist addition in HEK-293 cells expressing YFP-hDAT and myc-rKOPr. This was carried out by Western blotting for P-ERK1/2 followed by stripping the membrane and reprobing for ERK1/2. All compounds activated ERK1/2, with salvinorin A causing the fastest phosphorylation at $1 \mathrm{~min}$, and the other agonists causing ERK1/2 phosphorylation at $5 \mathrm{~min}$ (Figure 5.6a and $\mathrm{b}$ to Figure 5.9a and b). ERK1/2 is activated by direct phosphorylation by MEK. The selective MEK1/2 inhibitor U0126 should therefore inhibit ERK1/2 phosphorylation. Inhibition of MEK1/2 (20 $\mu \mathrm{M} \mathrm{U0126,} 30$ $\min$ ) attenuated the increase in P-ERK1/2, as measured by Western blotting (Figure $5.6 \mathrm{c}$ and $\mathrm{d}$ to Figure 5.9c and d).

All of the KOPr agonists investigated increased P-ERK1/2, and this phosphorylation was inhibited by U0126. Therefore, experiments investigating if inhibition of PERK1/2 causes an inhibition of the increase in DAT function caused by KOPr activation were conducted. A previous study showed that U0126 decreased cell surface expression of DAT (Moron et al. 2003), but at a much higher concentrations than those used in our study (50 $\mu \mathrm{M}$ compared to $20 \mu \mathrm{M}$ ); therefore, the effect of U0126 incubation on YFP-hDAT cell surface expression was also tested. We found that a 30 min incubation with U0126 in HEK-293 cells co-transfected with YFP-hDAT and mycrKOPr caused no decrease in YFP-hDAT cell surface expression on its own (Figure 5.5a). Initial $\mathrm{ASP}^{+}$uptake studies were carried out to find out whether U0126 on its own could directly lead to inhibition of $\mathrm{ASP}^{+}$uptake. Again, we found there was no change in uptake of $\mathrm{ASP}^{+}$at $200 \mathrm{~s}$ after U0126 incubation compared to controls (Figure 5.5b). 
$\mathrm{ASP}^{+}$uptake studies carried out after a 30 min incubation with U0126 showed a complete inhibition of the increase in $\mathrm{ASP}^{+}$uptake induced by all the KOPr agonists tested (Figure 5.6e to Figure 5.9e). This suggests that the ERK1/2 pathway is involved in the interaction between KOPr and DAT. 
A
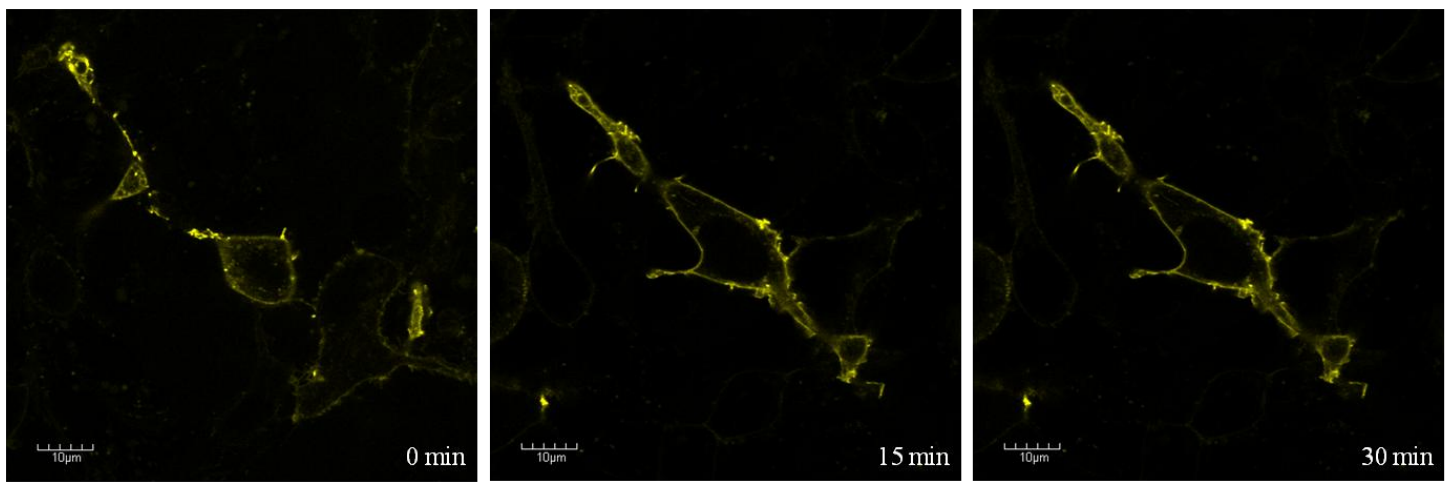

B

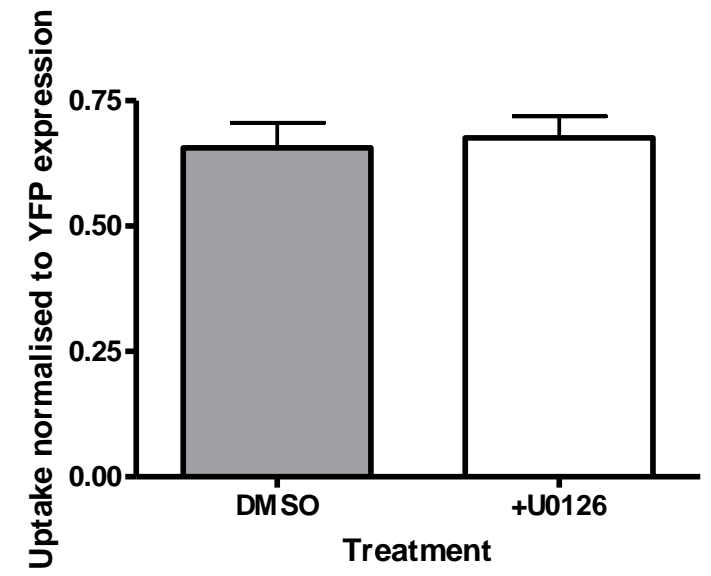

Figure 5.5: Effect of the MEK1/2 inhibitor U0126 on untreated HEK-293 cell. (A) YFP-hDAT fluorescence microscopy images of DAT- and KOPr-transfected HEK-293 cells untreated with KOPr agonists. A representative time-course of HEK-293 cells incubated with U0126 for $30 \mathrm{~min}$ is presented. There was no significant internalisation of YFP-hDAT by U0126. (B) Summary of results of $\mathrm{ASP}^{+}$uptake in cells incubated with U0126 for 30 min. No significant effect on $\mathrm{ASP}^{+}$uptake was seen $(\mathrm{n}=51-58$ cells, $p=0.7722$, Student's t-test). 

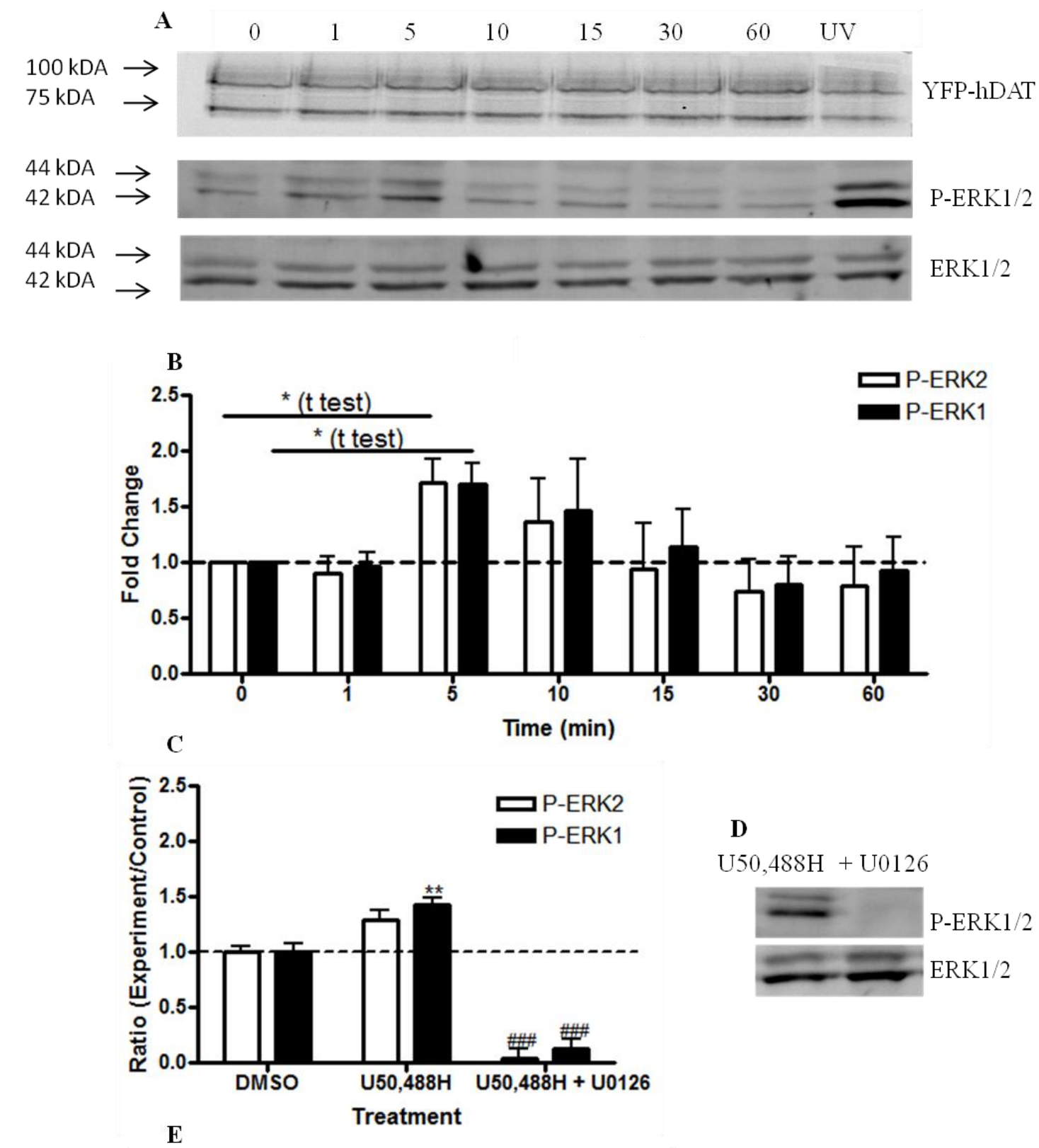

D

$\mathrm{U} 50,488 \mathrm{H}+\mathrm{U} 0126$

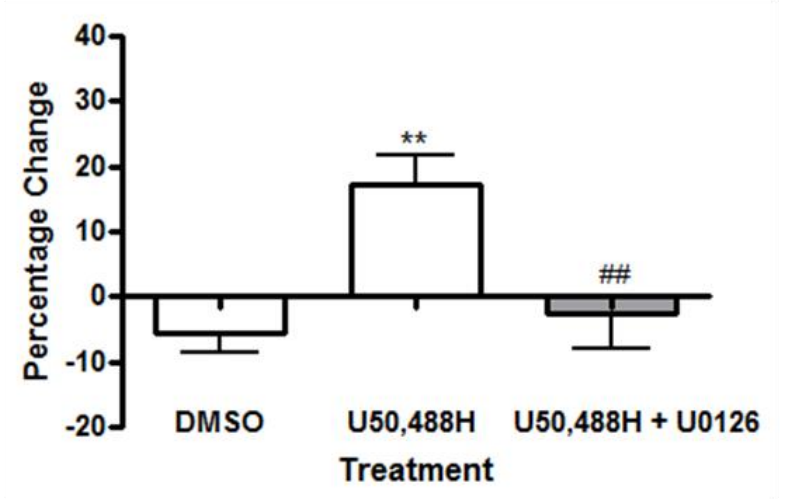


Figure 5.6: U50,488H modulates DAT function through an ERK1/2 dependent pathway. (A) Fluorescent scan of representative gel showing YFP-DAT expression (top), and representative Western blots showing P-ERK1/2 expression (middle) and ERK1/2 expression (bottom) after U50,488H treatment. UV (1 h) was used as a positive control for ERK1/2 activation but was not graphed. (B) P-ERK1/2 expression was normalised to ERK1/2 expression and expressed graphically as a fold change (mean \pm $\mathrm{SEM}$ ) compared to time 0. There was a significant increase in P-ERK1/2 at $5 \mathrm{~min}(\mathrm{P}-$ ERK1, $p=0.0101, \mathrm{P}$-ERK2, $\mathrm{p}=0.0104$, Student's t-test, $\mathrm{n}=4)(\mathrm{C}, \mathrm{E})$ Inhibition of the ERK1/2 activation by U50,488H occurred after a 30 min pre-treatment with U0126 (20 $\mu \mathrm{M}$ ) (One-way ANOVA followed by Bonferroni post test, $* * p<0.01$ compared to appropriate control, \#\#p<0.001 compared to appropriate U50,488H-treated control, $\mathrm{n}$ =6. (D) The increase in uptake caused by a $100 \mathrm{~s}$ incubation with $\mathrm{U} 50,488 \mathrm{H}$ was attenuated by U0126 (One-way ANOVA followed by Bonferroni post test, **p $<0.01$ compared to the DMSO control, \#\#p<0.01 compared to U50,488H treated control, $\mathrm{n}=$ 40-57). The addition of DMSO as a vehicle control had no effect on uptake compared to 0 (one-sample Student's t-test). 

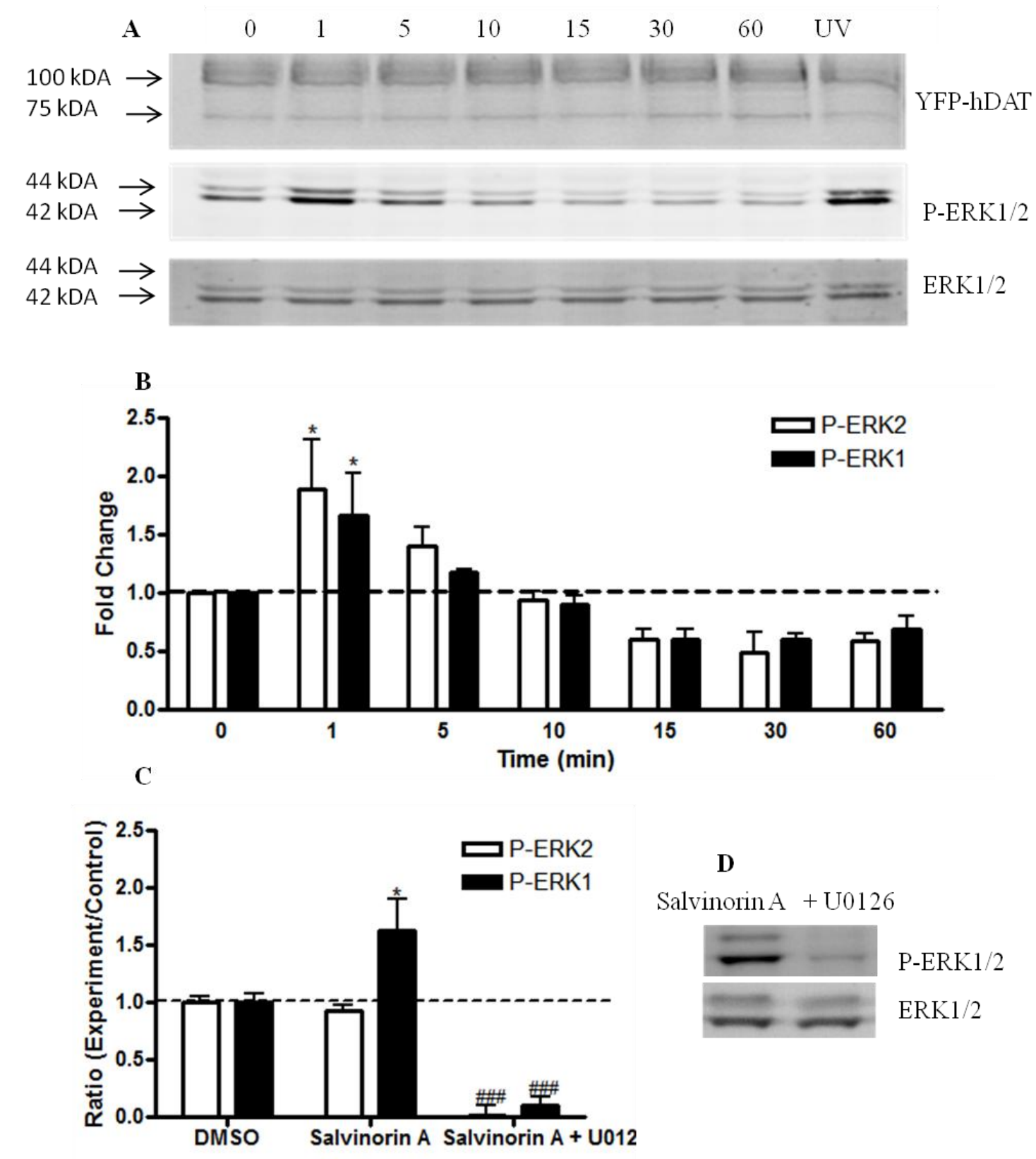

D

Salvinorin A + U0126

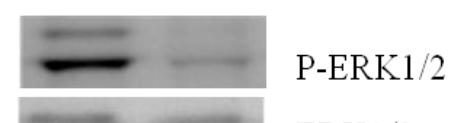

ERK1/2

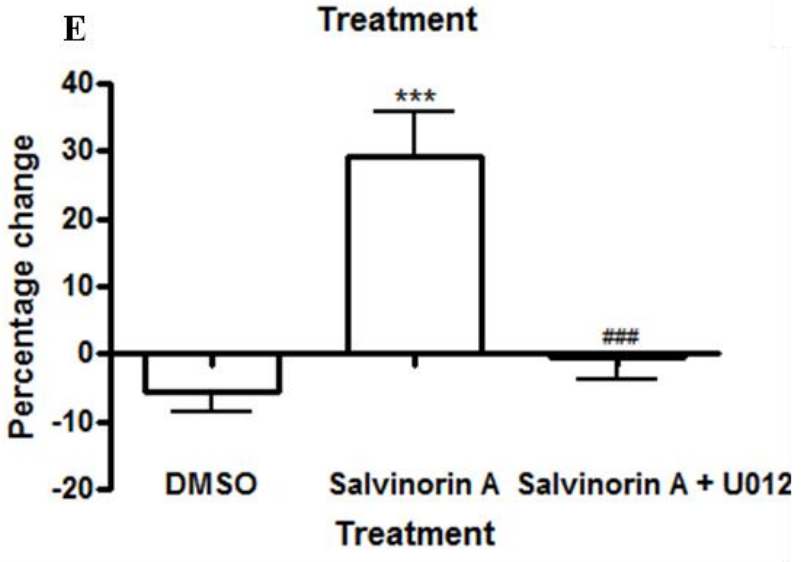


Figure 5.7: Salvinorin A modulates DAT function through an ERK1/2 dependent pathway. (A) Fluorescent scan of representative gel showing YFP-hDAT expression (top), representative Western blot membrane showing P-ERK1/2 expression (middle) and ERK1/2 expression (bottom) after salvinorin A treatment. UV (1 h) was used as a positive control for ERK1/2 activation but was not graphed. (B) P-ERK1/2 expression was normalised to ERK1/2 expression and expressed graphically as a fold change compared to time 0 . After $1 \mathrm{~min}$ there was a significant increase in P-ERK1/2 (Oneway ANOVA followed by Bonferroni post test, $\left.{ }^{*} \mathrm{p}<0.05, \mathrm{n}=4\right) \quad(\mathrm{C}, \mathrm{E})$ Inhibition of ERK1/2 activation by salvinorin A occurred after a 30 min pre-treatment with U0126 $(20 \mu \mathrm{M})$ (One-way ANOVA followed by Bonferroni post test, $* \mathrm{p}<0.01$ compared to appropriate control, \#\#\#p<0.001 compared to appropriate salvinorin A-treated control, $\mathrm{n}$ =6. (D) A representative Western blot of the effect of U0126 on the salvinorin Ainduced phosphorylation of ERK1/2. (E) The increase in uptake after a $60 \mathrm{~s}$ incubation with salvinorin A was attenuated by pre-treatment with U0126 (One-way ANOVA followed by Bonferroni post test, $* * * p<0.001$ compared to DMSO control, \#\#\#p<0.001 compared to salvinorin A-treated control, $\mathrm{n}=32-50$. The addition of DMSO as a vehicle control had no effect on uptake from 0 (one-sample Student's t-test). 
A
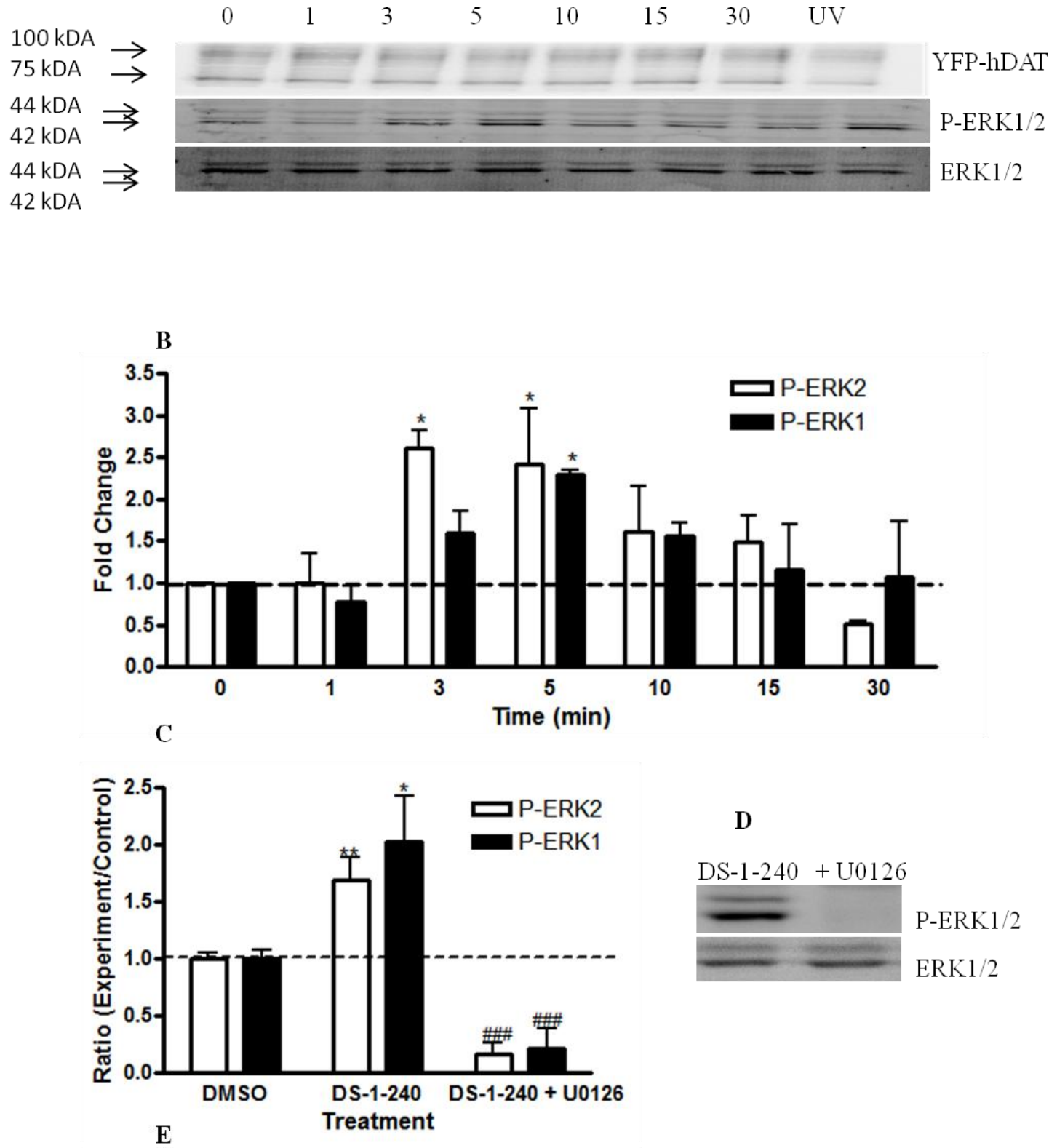

D

DS-1-240 + U0126

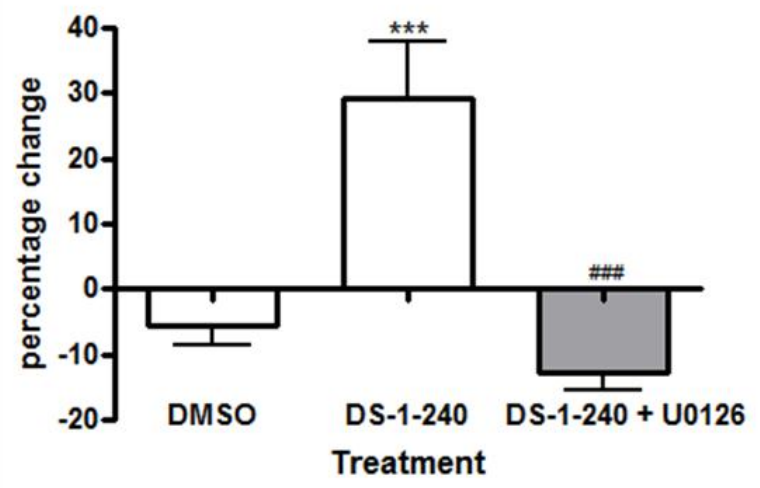

P-ERK1/2

ERK1/2 
Figure 5.8: DS-1-240 modulates DAT function through an ERK1/2 dependent pathway. (A) Fluorescent scan of representative gel showing YFP-hDAT expression (top), representative Western blot membrane showing P-ERK1/2 expression (middle) and ERK1/2 expression (bottom) after DS-1-240 treatment. UV (1 h) was used as a positive control for ERK1/2 activation but was not graphed. (B) P-ERK1/2 expression was normalised to ERK1/2 expression and expressed graphically as a fold change compared to time 0 . After 3 and $5 \mathrm{~min}$ there was a significant increase in P-ERK1/2 (One-way ANOVA followed by Bonferroni post test, $\left.{ }^{*} \mathrm{p}<0.01, n=4\right)$. (C,E) Inhibition of ERK1/2 activation by DS-1-240 occurred after pre-treatment with U0126 $(20 \mu \mathrm{M}, 30$ min) One-way ANOVA followed by Bonferroni post test, ${ }^{*} \mathrm{p}<0.05, * * \mathrm{p}<0.01$ compared to appropriate control, \#\#\#p<0.001 compared to appropriate DS-1-240 treated control, $\mathrm{n}$ =6. (D) A representative Western blot of the effect of U0126 on the DS-1-240-induced phosphorylation of ERK1/2. (E) The increase in uptake caused by a 200s incubation with DS-1-240 was attenuated after incubation with U0126. One-way ANOVA followed by Bonferroni post test, $* * * \mathrm{p}<0.001$ compared to DMSO control, \#\#\#p<0.001 compared to DS-1-240 treated control, $\mathrm{n}=32-50$, respectively. The addition of DMSO as a vehicle control did not lead to a significant decrease in uptake from 0 (One sample Student's t-test). 

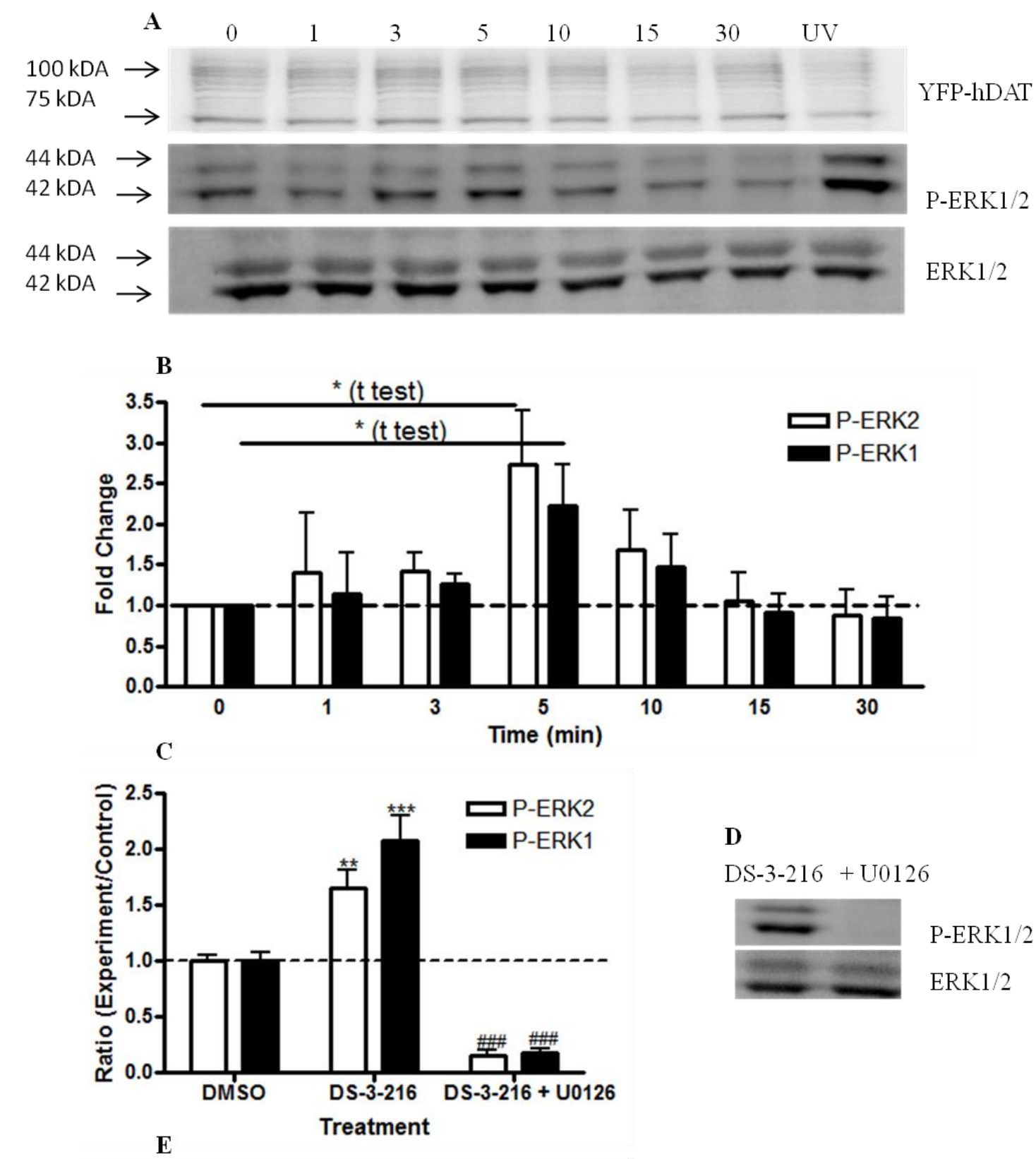

D

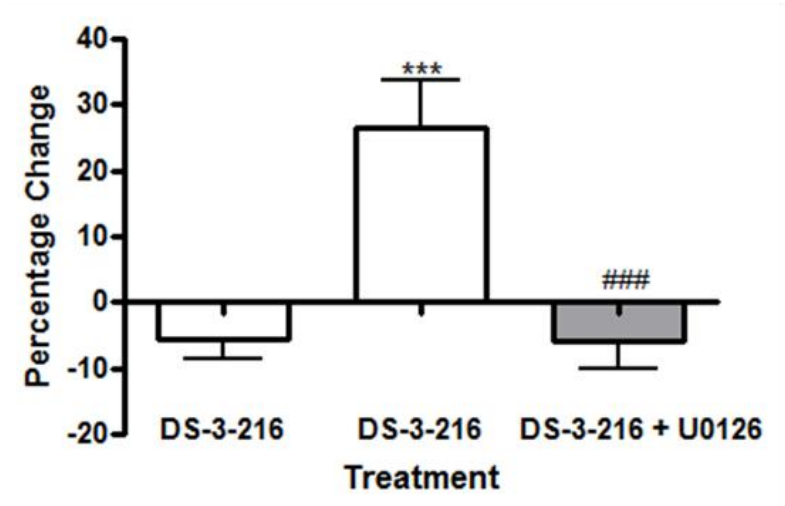

DS-3-216 + U0126

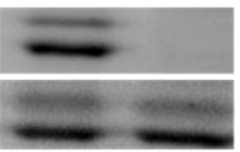

P-ERK1/2

ERK1/2 
Figure 5.9: DS-3-216 modulates DAT function through an ERK1/2 dependent pathway. (A) Fluorescent scan of representative gel showing YFP-hDAT expression (top), representative Western blot membrane showing P-ERK1/2 expression (middle) and ERK1/2 expression (bottom) after DS-3-216 treatment. UV (1 h) was used as a positive control for ERK1/2 activation but was not graphed. (B) P-ERK1/2 expression was normalised to ERK1/2 expression and expressed graphically as a fold change compared to time 0 (B). After 5 min there was a significant increase in P-ERK1/2 (PERK1, $\mathrm{p}=0.0496$, P-ERK2, $\mathrm{p}=0.0394$, student $\mathrm{t}$ test, $\mathrm{n}=4$ ) $\mathrm{C}$,E) Inhibition of ERK1/2 activation by DS-3-216 occurred after a 30 min pre-treatment with U0126 (20 $\mu \mathrm{M}$ ) (One-way ANOVA followed by Bonferroni post test, **p<0.01, ***p $<0.001$ compared to appropriate control, \#\#\#p<0.001 compared to appropriate DS-3-216 treated control, $n=6$ ). (D) A representative Western blot of the effect of U0126 on the DS-3216-induced phosphorylation of ERK1/2. (E) The increase in uptake caused by a $200 \mathrm{~s}$ incubation with DS-3-216 was attenuated after incubation with U0126. (One-way ANOVA followed by Bonferroni post test, $* * * p<0.001$ compared to DMSO control, $\# \# \# \mathrm{p}<0.001$ compared to $\mathrm{U} 50,488 \mathrm{H}$ treated control, $\mathrm{n}=40-57$ respectively). The addition of DMSO as a vehicle control did not lead to a significant decrease in uptake from 0 (One-sample Student's t-test). 


\subsubsection{ERK1/2 activation and cell surface expression of YFP-hDAT}

ERK1/2 activation leads to increased DAT function and also cell surface expression (Moron et al. 2003). Incubation with salvinorin A and U69,593 for 30 min caused an increase in cell surface expression of YFP-hDAT that was seen with both TIRFM and cell surface biotinylation techniques. We therefore investigated whether inhibition of ERK1/2 phosphorylation inhibited the increase in DAT cell surface expression seen with U69,593 and salvinorin A. For these experiments, cells were incubated with the MEK1/2 inhibitor U0126 $(20 \mu \mathrm{M})$ for $30 \mathrm{~min}$ before addition of salvinorin $\mathrm{A}$ or U69,593. Cell surface biotinylation was carried out after $30 \mathrm{~min}$ incubation with the KOPr agonist. TIRFM studies were carried out for a total period of $40 \mathrm{~min}$, with 10 images taken $1 \mathrm{~min}$ apart before KOPr agonist addition for baseline readings and then 30 images taken 1 min apart to capture any changes in cell surface expression.

Control experiments were carried out with U0126, and the two vehicles used for this study, ethanol (for U69,593) and DMSO (for salvinorin A). As Figure 5.10 shows, U0126 had no effect on cell surface expression as measured by TIRFM for both ethanol- (Figure 5.10a) and DMSO- (Figure 5.10c) treated cells. In cell surface biotinylation experiments, ethanol on its own caused a significant increase in intracellular YFP-hDAT $(\mathrm{p}<0.01$, with no change in cell surface expression (Figure $5.10 b$ ). With DMSO, there was no significant change in YFP-hDAT expression (Figure $5.10 \mathrm{~d})$.

Treatment of cells with U69,593 led to a non-significant increase of 7\% in cell surface expression using TIRF microscopy (Two-way ANOVA, data compared to vehicle data shown in Figure 5.11a) and a significant increase of $37 \%$ with cell surface biotinylation $(\mathrm{p}<0.01)$ compared to vehicle controls (Figure 5.11a-c). When the final data points for TIRFM (25 min to $29 \mathrm{~min}$ ) were compared between vehicle and U69,593 (Student's ttest), there was a significant increase in uptake $(\mathrm{p}<0.001)$. Pre-treatment for $30 \mathrm{~min}$ with U0126 (20 $\mu \mathrm{M})$ followed by U69,593 showed a small, non-significant decrease in cell surface expression of YPF-hDAT measured by TIRFM but a significant increase in cell surface expression by cell surface biotinylation $(\mathrm{p}<0.01)$. Overall, these data suggest that U0126 may not alter the change in cell surface expression seen with U69,593 after a $30 \mathrm{~min}$ incubation, and that the MEK/ERK pathway therefore may not be involved in the U69,593-induced increase in DAT cell surface expression. 
Treatment of cells with salvinorin A significantly increased YFP-hDAT cell surface expression as measured by TIRFM (Figure 5.12c) (compared to vehicle data shown in Figure 11c, two-way ANOVA, followed by Bonferroni post test, with vehicle versus salvinorin A showing significance; $\mathrm{p}<0.001)$. Treatment at $28 \mathrm{~min}$ showed a significant difference between vehicle (Bonferroni post test, $\mathrm{p}<0.05$ ). At $28 \mathrm{~min}$ there was also a significant difference between salvinorin A treatment and U0126 pre-treated salvinorin A using the Bonferroni post test $(\mathrm{p}<0.01)$. Cell surface biotinylation studies also showed a significant $18.5 \%$ increase in cell surface YFP-hDAT after a $30 \mathrm{~min}$ salvinorin A treatment $(\mathrm{p}<0.05)$. However, there was no significant change seen between cells treated with salvinorin A and those pre-treated with U0126 followed by salvinorin A. This may be due to the sample size being too small or the U0126 inhibition being less than complete.

Overall these data suggest that there may be differences in the signalling pathways used by U69,593 and salvinorin A in their regulation of cell surface expression of YFPhDAT, but the changes in surface expression do not appear to be large enough to reach significance. Further experiments are needed to verify this difference between the two compounds. 

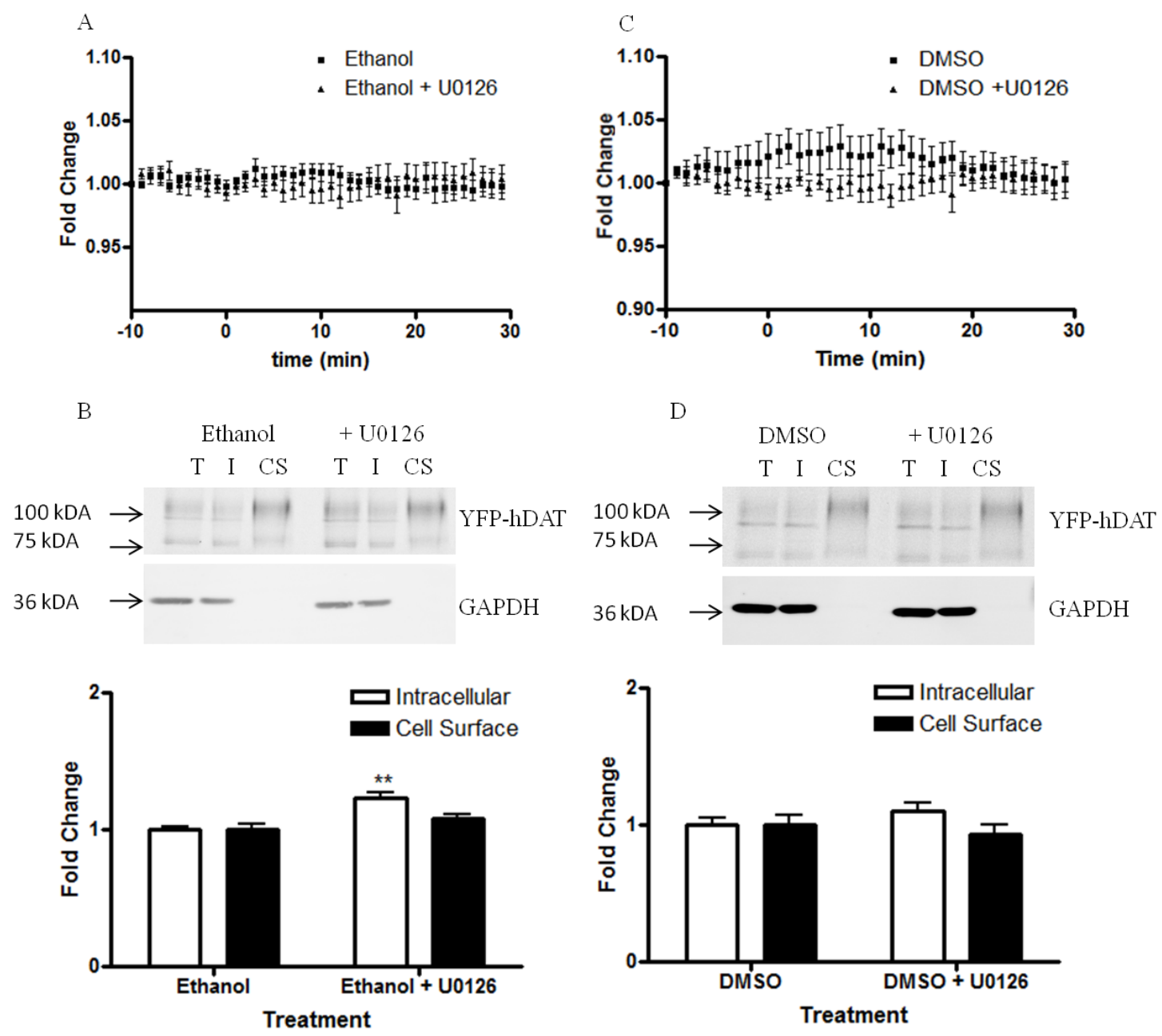

Figure 5.10: The effect of U0126 on the cell surface expression of YFP-hDAT in vehicle-treated HEK-293 cells. (A,C) In TIRFM experiments, after pre-treatment of cells for $30 \mathrm{~min}$ with U0126 $(20 \mu \mathrm{M})$, there was no effect of ethanol or DMSO on cell surface expression $(n=9-12)$. (B,D) In biotinylation experiments, there was no change in cell surface expression after DMSO in control or U0126 pre-treated cells; however, prior treatment with U0126 led to a small, but significant increase in the intracellular fraction following ethanol treatment $(n=6)$ (One-way ANOVA followed by a Bonferroni post test, $* * \mathrm{p}<0.01$. 

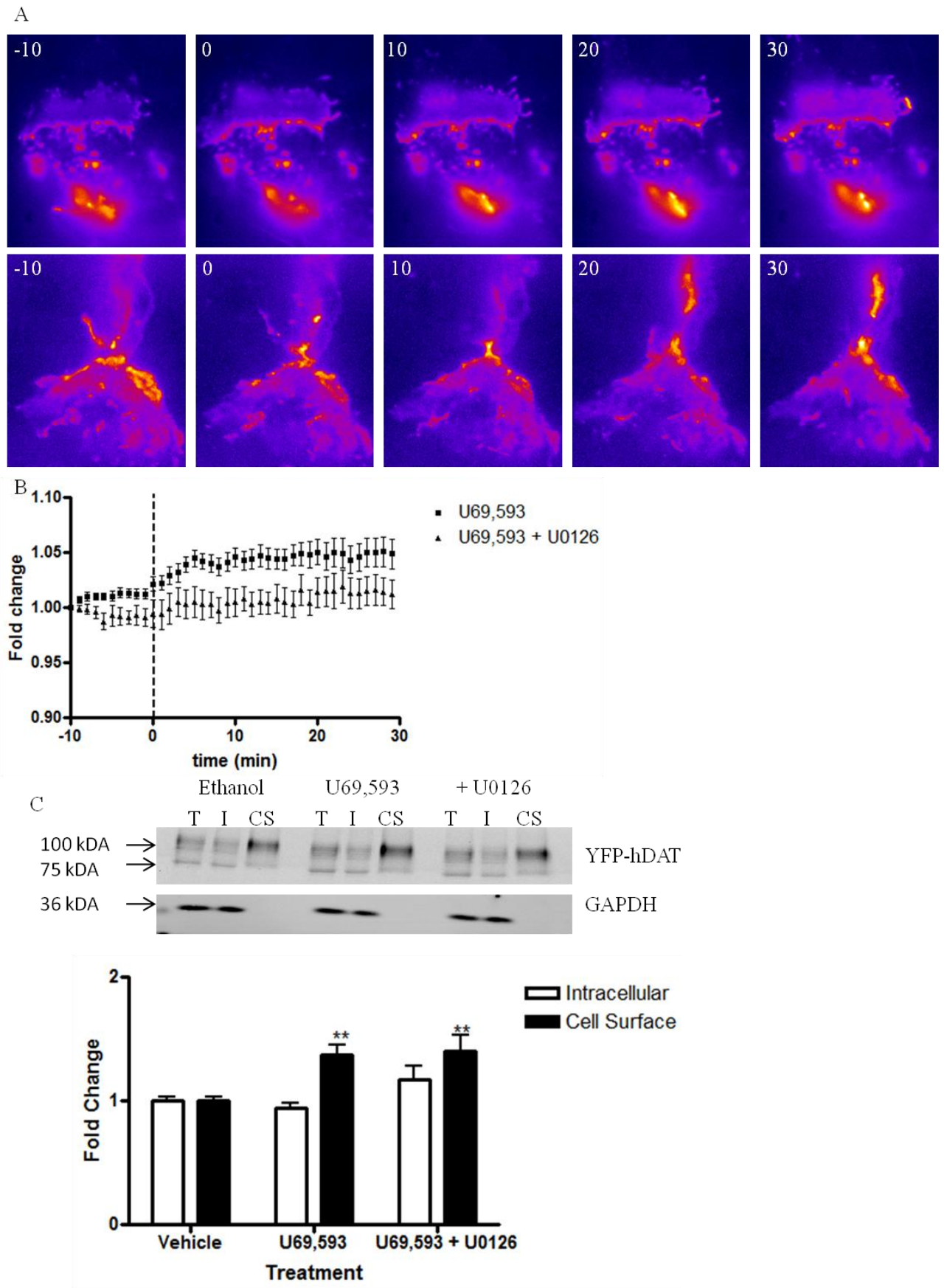

Figure 5.11: U0126 does not inhibit the increase in cell surface expression induced by U69,593. A) Representative TIRFM images at $-10,0,10,20$, and $30 \mathrm{~min}$ after U69,593 treatment. Treatments with U69,593 $(10 \mu \mathrm{M})$ (top row) and U69,593 after pre-treated with U0126 (bottom panel) are shown. B) U69,593 increased YFP-hDAT expression by $7 \%$ and the increase was not significantly different from cells pre-treated with U0126 (two-way ANOVA followed by Bonferroni post test, $n=10-16$ ). From 25$29 \mathrm{~min}$, the increase in cell surface expression after U69,593 treatment was significantly different from vehicle-treated samples (from figure 5.10a, $\mathrm{p}<0.001$, Student's t-test,). C) Cell surface biotinylation experiments showed a significant increase in cell surface expression of YFP-hDAT after a 30 min incubation with U69,593, and this increase was not inhibited by pre-treatment with U0126 (One-way ANOVA followed by a Bonferroni post test, $* * \mathrm{p}<0.01$ compared to vehicle control, $\mathrm{n}=6$ ). $\mathrm{T}=$ total, $\mathrm{I}=$ internal, $\mathrm{CS}=$ cell surface. 

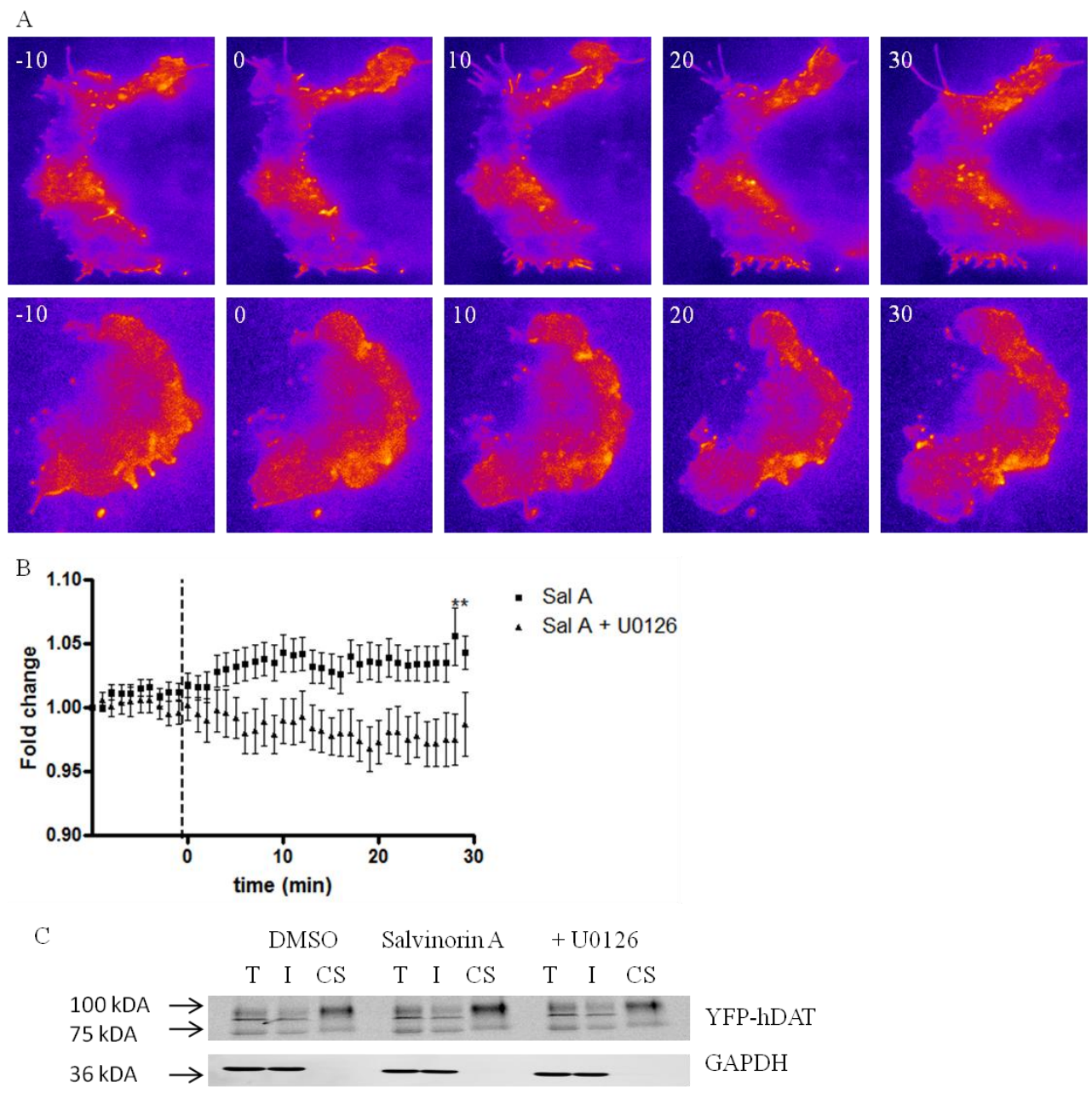

YFP-hDAT

GAPDH

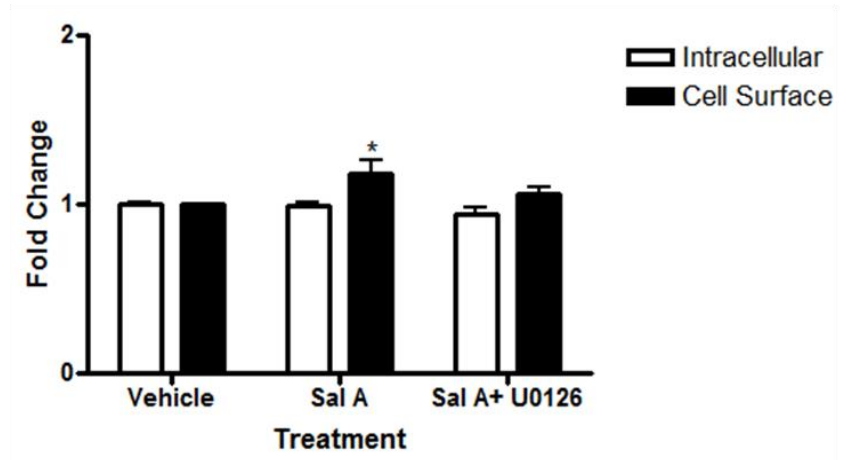

Figure 5.12: U0126 inhibited the salvinorin A-induced increase in cell surface expression. A) Representative TIRFM images at $-10,0,10,20$, and $30 \mathrm{~min}$ after salvinorin A treatment. Treatments with salvinorin A $(10 \mu \mathrm{M})$ (top row) and salvinorin A after pre-treated with U0126 (bottom panel) are shown. B) TIRFM experiments showed that salvinorin A significantly increased YFP-hDAT expression by $5.6 \%$, compared to vehicle control (from figure 5.10c, two-way ANOVA followed by Bonferroni post test, $\mathrm{p}<0.001$; at $28 \mathrm{~min}$ ). This increase was also significantly different from salvinorin A cells pre-treated with U0126 ( $* * p<0.01$, Student's t-test, $n=10-20$ ). C) In cell surface biotinylation experiments, salvinorin A (30 min) caused a significant increase in cell surface expression of YFP-hDAT, which was not observed after U0126 pre-treatment. However, there was no significant difference between the salvinorin A treated cell surface fraction and salvinorin A after pre-treatment with U0126 (One-way ANOVA followed by a Bonferroni post test, ${ }^{*} \mathrm{p}<0.05$ compared to vehicle control, $\mathrm{n}=$ 6). $\mathrm{T}=$ total, $\mathrm{I}=$ internal, $\mathrm{CS}=$ cell surface. 


\subsection{Discussion}

Both KOPr and DAT can be found within lipid rafts (Xu et al. 2006; Adkins et al. 2007; Foster et al. 2008) but no studies have been conducted on the effects of lipid raft disruption on the interaction between the two. As KOPr activation leads to an initial increase in DAT function (Chapter 4), the effect of lipid raft disruption and inhibition of the ERK1/2 pathway on DAT function was investigated. A 30 min incubation with salvinorin A or U69,593 also increased cell surface expression in transfected HEK-293 cells (Chapter 4), and therefore the effect of ERK1/2 inhibition was also investigated to see if this increase in cell surface expression was facilitated through this pathway. Experiments to determine if total YFP-hDAT protein expression changed over a $30 \mathrm{~min}$ incubation with these compounds were also carried out. It was found that total YFPhDAT protein did not change after a 30 min incubation with the KOPr agonists. The disruption of lipid rafts led to inhibition of the increase in function of YFP-hDAT at acute time points, and all the KOPr agonists tested caused activation of ERK1/2. Inhibition of ERK1/2 inhibited the increased YFP-hDAT transport function seen after an acute incubation with all of the KOPr agonists. ERK1/2 inhibition also appeared to prevent the effect of a 30 min incubation with salvinorin A on increased YFP-hDAT expression at the cell surface, but did not inhibit the effect of U69,593. This suggests that the increase in cell surface expression observed by these two compounds may occur through different pathways.

\section{$\underline{5.5 .1 \text { Total protein }}$}

One of the ways in which protein cell surface regulation could occur is through increases in total protein within the cell (Meiergerd et al. 1994a). As salvinorin A, U69,593, and DS-3-216 all caused increased cell surface expression of YFP-hDAT in HEK-293 cells co-transfected with myc-rKOPr and YFP-hDAT, the effect of these compounds on YFP-hDAT total protein was investigated after a $30 \mathrm{~min}$ incubation with the compounds. It was found that none of the KOPr agonists studied caused significant changes in YFP-hDAT total protein in these cells (Figure 5.1), suggesting that this is not the cause for the increased cell surface expression seen with some of the KOPr agonists. 


\section{$\underline{\text { 5.5.2 Lipid raft disruption }}$}

Previous studies have identified that HEK-293 cells contain cholesterol-rich regions, thought to be lipid rafts (O'Connell and Tamkun 2005; Adkins et al. 2007). The disruption of lipid rafts inhibited the effect of the KOPr agonists on DAT function. It has previously been reported that lipid raft disruption leads to an increase in KOPr agonist effects on KOPr (Xu et al. 2006) and a decrease in DAT function (Adkins et al. 2007; Foster et al. 2008). Interestingly, we found that treatment of cells with M $\beta C$ led to an overall increase in uptake of $\mathrm{ASP}^{+}$in both non-transfected and transfected cells (Figure 5.2). When non-specific $\mathrm{ASP}^{+}$uptake (non-transfected cells) was removed from the transfected cell uptake, there was a significant increase in $\mathrm{ASP}^{+}$uptake of $23 \%$ in the $\mathrm{M} \beta \mathrm{C}$-treated group (Table 5.1). As the non-specific uptake included the effects due to increased permeability caused by $\mathrm{M} \beta \mathrm{C}$ treatment, this effect would be expected to be due to increased specific uptake by YFP-hDAT. This is opposite to that reported from the two previous studies, that showed that M $\beta C$ decreased DAT function by $16 \%$ and 30\% (Adkins et al. 2007; Foster et al. 2008). Both of these studies used [ $\left.{ }^{3} \mathrm{H}\right] \mathrm{DA}$ uptake and also used different cell lines, and; therefore, the difference between theirs and our results may be due to the technique and cells used. $\mathrm{M} \beta \mathrm{C}$ treatment has also been shown to affect cell cytoskeletal components such as actin (Kenworthy et al. 2004). As DAT is also found in non-lipid raft environments there is the possibility that M $B C$ treatment caused changes in function of these non-lipid raft transporters as well.

Confirmation of lipid raft disruption was carried out using FRAP (Figure 5.3). We found that depletion of cholesterol by $\mathrm{M} \beta \mathrm{C}$ caused an increase in $\mathrm{t}_{1 / 2}$ and no change to Mf. This microscopy technique measures the movement of diffusing fluorescentlytagged proteins into a photobleached region of the cell membrane, either from lateral diffusion in the cell membrane or exocytosis from inside the cell. This technique has the ability to measure the kinetics of diffusion, giving $\mathrm{t}_{1 / 2}$ values (rate of movement back into the bleached region) and Mf values (total recovery of the bleached region). Another parameter, $\mathrm{D}$, the diffusion coefficient, is calculated from $\mathrm{t}_{1 / 2}$ and the radius of the bleach region. It requires the first post-bleach image to be taken immediately after bleaching has finished (Snapp et al. 2003). This coefficient is calculated so that experiments carried out using different equipment can be directly compared, and is generally published instead of $t_{1 / 2}$ values. The confocal microscope used in this study does not have the capability to measure $\mathrm{D}$ as it is not equipped with a tornado system and therefore cannot take a post-bleach image immediately after the bleach due to 
zooming in and out. Thus, in our study, the change in $\mathrm{t}_{1 / 2}$ cannot be directly compared to previously published data. But an increase in $\mathrm{t}_{1 / 2}$ does suggest that diffusion of YFPhDAT in $\mathrm{M} \beta \mathrm{C}$-treated cells occurs more slowly than in control cells. As $\mathrm{t}_{1 / 2}$ is dependent on the surrounding environment of the cell and cell trafficking, a decreased $\mathrm{t}_{1 / 2}$ suggests that the membrane environment has become more difficult to diffuse through. The only other study that has investigated YFP-hDAT movement in HEK-293 cells using FRAP published only D values, which in their study increased after M $\beta C$ treatment (Adkins et al. 2007). An increased D value suggests an increase in protein mobility, which is the opposite of that seen in our study. One possibility for the difference between their study and ours is that the bleach region used in our study was larger than that of the Adkins et al. study, which had an average bleach region of 1-3 $\mu \mathrm{M}$ (Adkins et al. 2007). As the bleach region used in our study was larger, it would have included more non-lipid raft cell membrane compared to the previous study, and $\mathrm{M} \beta \mathrm{C}$ has been shown to also affect proteins within non-lipid raft environments due to its interaction with the actin cytoskeleton (Kenworthy et al. 2004). Although our result disagrees with the only other DAT study, there have been many other studies that have shown decreased mobility (decreased D) after lipid raft disruption for other membrane proteins, found both within lipid and non-lipid environments (Kenworthy et al. 2004; O'Connell and Tamkun 2005; Vrljic et al. 2005; Nishimura et al. 2006; Baier et al. 2010). For example, nAChR mobility decreased in $\mathrm{CHO}$ cells after $\mathrm{M} \beta \mathrm{C}$ treatment (Baier et al. 2010). This decrease was thought to be due to cholesterol depletion leading to solid phase clumps forming in the membrane, as other studies on MHC II receptors and voltage gated potassium channels have reported the formation of these clumps (O'Connell and Tamkun 2005; Nishimura et al. 2006). It has also been demonstrated that increased cholesterol content in model lipid bilayers leads to increased fluidity of the membrane by interfering with tight packing of the phospholipid fatty acid tails, supporting the observation that decreasing cell membrane cholesterol would lead to decreased mobility (Korlach et al. 1999).

There is a lot of disagreement about whether depletion of cholesterol from the cell membrane should lead to increased or decreased mobility of lipid raft proteins. A decrease in mobility for lipid raft proteins may be due to a decrease in the cholesterolphospholipid complex, leading to the formation of solid-like phospholipid regions within the cell membrane, decreasing mobility (Nishimura et al. 2006); whereas, an 
increase in mobility of lipid raft proteins may be due to disbanding of solid lipid rafts, freeing lipid raft proteins (Adkins et al. 2007).

As the $\mathrm{ASP}^{+}$uptake experiments carried out in our study measure the changes in uptake before and after KOPr agonist addition within the same cell, it allows for any effect of $\mathrm{M} \beta \mathrm{C}$ on YFP-hDAT function to be disregarded as the cell is still capable of $\mathrm{ASP}^{+}$ uptake and the experiment is analysed using a within-cell design. Prior incubation of cells with $\mathrm{M} \beta \mathrm{C}$ led to an inhibition of the effect of the KOPr agonists on YFP-hDAT function (Figure 5.4). This could be for a number of reasons: first, the disruption of the lipid rafts may have interfered with the interaction between KOPr and DAT. This could be either through inhibition of one of the pathways needed to communicate between the two, or directly by inhibiting a physical interaction between the two proteins. Alternatively, lipid raft disruption could lead to a conformation change in KOPr or DAT, preventing interactions from occurring and blocking the KOPr-mediated increase in DAT function. As $\mathrm{M} \beta \mathrm{C}$ lipid raft disruption leads to an increase in the effect of $\mathrm{U} 50,488 \mathrm{H}$ on $\mathrm{KOPr}$, we expect that it is more likely that disruption of lipid rafts would lead to conformational changes of YFP-hDAT that may prevent its coupling to KOPr or remove its ability to be regulated by KOPr-activated pathways.

Overall, lipid raft disruption inhibited the effect of KOPr agonists on DAT function. Lipid raft disruption by $\mathrm{M} \beta \mathrm{C}$ also led to a decrease in DAT mobility as measured by FRAP. Interestingly, lipid raft disruption also appeared to increase DAT function in these cells, which is something that needs to be investigated further.

\subsubsection{ERK1/2 pathway}

The ERK1/2 pathway has been implicated in the regulation of DAT function and cell surface expression (Zapata and Shippenberg 2002; Moron et al. 2003; Bolan et al. 2007) and is also known to be activated by KOPr agonism (Belcheva et al. 2005). Stimulation of the DRD2 and D3 receptors increases function of DAT by increasing DAT cell surface expression through an ERK1/2 dependent pathway (Zapata and Shippenberg 2002; Bolan et al. 2007). Activation of ERK1/2 is also involved in the rewarding effects of drugs of abuse. For example, cocaine-induced hyperlocomotion in laboratory rats was inhibited by treatment with ERK1/2 inhibitors (Valjent et al. 2000). In the present study, we investigated the effects of the KOPr agonists on ERK1/2 phosphorylation, and the effect of ERK1/2 inhibition on DAT function. All the KOPr 
agonists led to increased P-ERK1/2 between 1 and 5 min after addition of the agonist (Figure 5.6 to 5.9). This increase was inhibited by the MEK1/2 inhibitor U0126. U0126 also inhibited the effect of these agonists on $\mathrm{ASP}^{+}$uptake into DAT/KOPr transfected cells. This demonstrates that the ERK1/2 pathway is involved in the interaction between KOPr and DAT in these cells. There has been only one reported study that has investigated the effect of DS-3-216 on ERK1/2 activation in primary and immortalised rat astrocytes (McLennan et al. 2008). A significant increase in ERK1/2 phosphorylation was seen from 3 to $15 \mathrm{~min}$ in primary astrocytes and 5 to $10 \mathrm{~min}$ in immortalised astrocytes, with a peak occurring in both cell types at $5 \mathrm{~min}$. In our study DS-3-216 led to a significant increase in P-ERK1/2 after $5 \mathrm{~min}$, which then rapidly dropped off until levels at $30 \mathrm{~min}$ were the same as the control. Therefore, it appears that the activation in HEK-293 cells is short-lived compared to those in primary astrocyte cultures. This is most likely due to differences in endogenous levels of proteins required for this signalling pathway, leading to different activation patterns in HEK-293 cells compared to glial cells. A previous study by Bruchas et al. (2006) showed that U50,488H also caused significant ERK1/2 activation in AtT-20 pituitary tumour cells (Bruchas et al. 2006). The increase peaked $15 \mathrm{~min}$ after U50,488H addition and was significantly increased for up to $30 \mathrm{~min}$. Their study used $10 \mu \mathrm{M}$ () $\mathrm{U} 50,488 \mathrm{H}$, which is the more active form of $\mathrm{U} 50,488 \mathrm{H}$; whereas, our study used 10 $\mu \mathrm{M}$ (+/-)U50,488H. Therefore, even though we used the same concentration of the agonist, the potency would be less than that of Bruchas et al. which may lead to weaker activation of ERK1/2.

Complete inhibition of ERK1/2 by U0126 was obtained at the concentration used (Figure 5.6 to 5.9), and this led to an inhibition of the increased function of DAT following KOPr activation (Figure 5.6 to 5.9). Previous studies carried out investigating DAT and ERK1/2 have shown that inhibition of ERK1/2 by the MEK inhibitors U0126 and PD98059 led to a decrease in DAT function. This was found to be due to decreased cell surface expression of DAT in rat striatal synaptosomes and EM4 cells expressing FLAG-hDAT (Moron et al. 2003). In our study, incubation of HEK-293 cells expressing YFP-hDAT and myc-rKOPr did not lead to decreased cell surface expression of YFP-hDAT or to a decrease in function (Figure 5.4). This is most probably due to the lower concentration of MEK inhibitor used in our study $(20 \mu \mathrm{M})$ compared to the $50 \mu \mathrm{M}$ used in the previous study (Moron et al. 2003). Interestingly, ethanol following U0126 treatment caused a small increase in intracellular levels of 
YFP-hDAT (Figure 5.10). However, as this increase was not observed with TIRFM or with the DMSO vehicle control, it was probably due to an artefact of the cell surface biotinylation studies. Biotin is unable to bind to proteins found within cell membrane invaginations, so proteins in these invaginations are classed as being part of the intracellular fraction, rather than the cell surface fraction, and this may account for this observation.

Overall, addition of KOPr agonists led to an increase in active ERK1/2 between 1-5 min after addition, and inhibition of ERK1/2 led to complete inhibition of the increase in DAT function due to KOPr activation, thus, demonstrating that the ERK1/2 pathway is involved in the interaction between KOPr and DAT.

\subsubsection{Cell surface expression studies and ERK1/2}

DAT cell surface regulation can be controlled by ERK1/2 phosphorylation (Moron et al. 2003). In our study, we investigated whether inhibition of ERK $1 / 2$ activation could inhibit the increase in YFP-hDAT cell surface expression seen after 30 min with U69,593 or salvinorin A. Experiments were carried out with addition of salvinorin A and U69,593 as positive controls (Figure 5.11 and 5.12). Cell surface biotinylation data showed similar significant increases in cell surface expression as shown in the data of Chapter 4 (U69,593: increase of $37 \%$ in both chapters, salvinorin A: increase of $25 \%$ in Chapter 4 and $18.5 \%$ in this chapter). TIRFM experiments also showed similar increases in cell surface expression after U69,593 treatment of $7 \%$ in this chapter, compared to $11.5 \%$ in chapter 4 , and for salvinorin A of $5.6 \%$ in this chapter compared to $9.3 \%$ in chapter 4 . Both of these treatments led to significant increases at later time points compared to vehicle control (Figure 5.10, 5.11, 5.12). The differences in the magnitude of the effects between the two different techniques are discussed in Chapter 4.

In TIRFM studies, treatment of cells with U0126 before U69,593 reduced the KOPr agonist's induction of YFP-hDAT cell surface expression, however, no inhibition of the effect of U69,593 was seen in cell surface biotinylation studies. This suggests that there may still be increases in cell surface expression after U69,593 treatment when the ERK1/2 pathway is completely inhibited. Inhibition of ERK1/2 signalling by U0126 significantly inhibited the effect of salvinorin A on YFP-hDAT cell surface expression at 30 min using TIRFM. Cell surface biotinylation data showed an inhibition of the 
effect of salvinorin A, however, there was no significant difference between U0126 non- and pre-treated samples. As the effect of salvinorin A was inhibited by ERK1/2 inhibition but the effects of the classic KOPr agonist U69,593 were not, these results suggest that salvinorin A regulates DAT cell surface expression through a different pathway than U69,593.

Overall these data suggest that the classic KOPr agonist U69,593 and the novel KOPr agonist salvinorin A may work through different pathways. As these compounds appear to have slightly different modes of action on DAT, compounds based on the structure of salvinorin A may have good potential for development as pharmaceuticals for treatment of addiction as they may have decreased or at least different side effects as a result of the different pathways they activate. 


\subsection{Conclusion}

This chapter demonstrated that KOPr and DAT interact through the ERK1/2 pathway and that lipid rafts are also likely to play a role in the interaction between the two. Treatment with the MEK1/2 inhibitor U0126 inhibited the increase in DAT function caused by KOPr activation. U0126 also inhibited the salvinorin A-induced increase of DAT cell surface expression, but did not appear to inhibit the effects of U69,593, suggesting that these compounds may act through different pathways. Disruption of lipid rafts by the cholesterol depleter $\mathrm{M} \beta \mathrm{C}$ also prevented the $\mathrm{KOPr}$ agonist-induced increases in $\mathrm{ASP}^{+}$uptake, supporting the hypothesis that lipid rafts play an important role in regulation of DAT by KOPr. 


\section{Chapter 6: Final Discussion}

\subsection{Significance and key findings}

\subsubsection{Significance}

Drug addiction is a major social and economic issue in New Zealand, and current therapeutic treatments often have poor long-term success rates or are addictive themselves. There are also no FDA approved pharmacotherapies available for psychostimulants such as cocaine and methamphetamine. Relapse is also an issue, with $80 \%$ of drug addicts relapsing at least once during their lifetime (Shippenberg et al. 2007). There is therefore a need for further investigation into pharmacotherapies for drug addiction to improve success rates and decrease side effects, and to treat addiction for all drugs of abuse.

DAT plays an important role in the brain's natural reward pathway and is a major target for drugs of abuse such as cocaine and amphetamines. Previous studies have shown that acute KOPr activation attenuates the effects of drugs of abuse in laboratory animals (Schenk et al. 1999; Collins et al. 2001a; Morani et al. 2009), and there is evidence that this occurs partially through increased DAT function (Thompson et al. 2000). Treatment with KOPr agonists can also lead to side effects such as depression and dysphoria (Redila and Chavkin 2008), which implicate the 5-HT system in their mode of action. KOPr antagonists show anti-depressant effects (Mague et al. 2003) and prevent stress-induced reinstatement in animal models of addiction (Beardsley et al. 2005; Carey et al. 2007). This further suggests that modulation of the 5-HT system occurs via KOPr and demonstrates the potential use of KOPr ligands and peptides for pharmacotherapies. Although there have been several KOPr agonists studied clinically as anti-addictive pharmacotherapies there has been little or no success, most likely due to the low dose used to avoid side effects (Walsh et al. 2001b; Preston et al. 2004). This suggests that, while promising, classic KOPr agonists may not be useful as pharmacotherapies for drug addiction. Therefore, given the success in animal models of addiction, research carried out on novel KOPr agonists still has the potential to provide successful pharmacotherapies without the negative side effects seen with the classic agonists.

The novel KOPr agonist salvinorin A differs from the classical agonist by having a nonnitrogenous structure. Therefore, salvinorin A and its analogues provide a new family of KOPr agonists to study as potential pharmacotherapies. It is hoped that their side 
effect profiles will differ and be less disruptive than what is seen with the classic agonists (Roth et al. 2002; Prisinzano et al. 2005). Investigating the effects of salvinorin A and its analogues on DAT and SERT will provide more information on how this novel agonist functions, and this information may help lead to successful pharmacotherapies for drug addiction. KOPr antagonists which are also based on the structure of salvinorin A may also lead to pharmacotherapies for depression and help to prevent stress-induced relapse to drug taking.

\section{$\underline{6.1 .2 ~ K e y ~ f i n d i n g s ~}$}

The key findings of this study are summarised in Table 6.1.

\subsubsection{KOPr modulates DAT and SERT in isolated rat brain tissue}

The KOPr agonists U50,488H, U69,593, salvinorin A, DS-1-240, and DS-3-216 all increased DAT function in the striatum, NAc, and medial PFC of drug naïve rat brains. Function was measured by RDEV. Salvinorin A had a faster onset of action (1 min) compared to the other agonists investigated, which increased uptake only after $4 \mathrm{~min}$ exposure. For all compounds studied, there was an increase in Vmax. This suggests that the increase in uptake seen may be partially due to increases in cell surface expression of DAT. Uptake of DA after salvinorin A treatment (500 nM) produced a linear substrate-velocity plot, which did not allow Vmax and $\mathrm{Km}$ values to be extrapolated. This may be due to the salvinorin A being metabolised or degraded, and fouling the electrode, thus preventing accurate measurements. The increase in uptake seen with all compounds was inhibited by prior incubation with the KOPr antagonist nor-BNI, confirming that this effect was KOPr-mediated. These data support previous unpublished data demonstrating the effect of acute U69,593 and salvinorin A treatment on DAT function in the rat NAc (T. Shippenberg, unpublished data), and also demonstrate that the novel KOPr agonists DS-3-216, and DS-1-240 and U50,488H act in a similar manner. The results also confirm that KOPr activation increases DAT function, resulting in a decrease in DA in the synapse, rather than just targeting DA receptors to prevent release.

Two of the most common causes of relapse to drug taking are stress and depression (Reviewed in Bruchas et al. 2010), and both the KOPr and the 5-HT system are involved in these pathways (Land et al. 2009; Goldman et al. 2010). As previous studies have demonstrated that KOPr activation attenuates drug seeking through a mechanism involving 5-HT (Zakharova et al. 2008a; Ruedi-Bettschen et al. 2010), the 
effect of acute KOPr agonists on SERT function was measured using RDEV. The establishment of RDEV to measure SERT function allowed the investigation of the effect of the KOPr agonists U50,488H, salvinorin A, and DS-1-240 on SERT transport in the rat striatum. These three KOPr agonists caused decreased SERT function after 15 min treatment. Thus, KOPr, in addition to stimulating DAT function, is also capable of decreasing SERT function. This is important because of the important role of SERT in depression and stress, which are both major causes of relapse.

Overall these data confirm previous studies that KOPr can regulate both DAT and SERT in specific rat brain regions. As all of these compounds have shown antiaddictive properties in animal models (Morani et al. 2009, A. Morani, unpublished data), the results of this study provides information as to how these compounds may be functioning to produce these effects. The increase in uptake of DA by DAT would contribute to the decrease in drug seeking behaviour observed in animals pre-treated with a KOPr agonist before drug-induced reinstatement tests. The decreased uptake of 5-HT after KOPr agonism may help decrease the negative depressive behaviour observed during withdrawal from drug taking and help to prevent relapse back to drug taking.

\subsubsection{KOPr modulates DAT in cells co-transfected with YFP-hDAT and myc-rKOPr}

These experiments used cell models, as they allow greater manipulation compared to animal models. The effects of KOPr agonists on $\mathrm{ASP}^{+}$uptake by DAT was investigated in HEK-293 cells, N2A cells, and COS7 cells. All of the compounds investigated gave dose-dependent increases in $\mathrm{ASP}^{+}$uptake by YFP-hDAT in all cell lines tested, and this uptake was inhibited by both the KOPr antagonist nor-BNI and the $\mathrm{G}_{\mathrm{i} / \mathrm{o}}$ inhibitor PTX. These results confirmed that the effect of these compounds was due to activation of KOPr and occurs through a $G_{i / o}$ specific pathway. In this model cell system, salvinorin A also showed a much shorter lag before a response was seen than the other compounds, with the maximal increase in $\mathrm{ASP}^{+}$uptake seen after only $60 \mathrm{~s}$. This matches previous work which showed that salvinorin A has a very fast onset of action (Hooker et al. 2008) and confirms what we found in our RDEV study. The effect of a 30 min incubation of drugs in HEK-293 cells was also investigated. U50,488H and salvinorin A caused a significant increase in DAT function, but the novel analogues DS1-240 and DS-3-216 had no effect. This demonstrates some inherent differences between salvinorin A and its analogues in addition to differences in potency. 
Overall these data confirm the use of cell models and $\mathrm{ASP}^{+}$uptake techniques as an effective technique for accurately measuring changes in YFP-hDAT function due to KOPr activation. Results gained in this part of the study match data gained in our RDEV study, and suggest that further investigation of how these compounds regulate DAT is warranted.

\subsubsection{KOPr modulation of the cell surface expression of DAT}

Since one of the major ways in which DAT function is regulated is through changes in cell surface expression, the effect of the KOPr agonists were investigated on cell surface expression of YFP-hDAT in HEK-293 cells. Acute incubations with KOPr agonists had no effect on YFP-hDAT cell surface expression, measured either by cell surface biotinylation or TIRF techniques. $\mathrm{ASP}^{+}$binding studies found a non-significant increase of $\mathrm{ASP}^{+}$binding to YFP-hDAT with U50,488H, salvinorin A, and DS-3-216, and a significant increase in binding after DS-1-240 treatment. This suggests that inactive DAT on the cell surface is activated by KOPr agonism, leading to increased binding and uptake of $\mathrm{ASP}^{+}$. After a 30 min incubation, U69,593 and salvinorin $\mathrm{A}$ significantly increased YFP-hDAT cell surface expression measured by both techniques, and DS-3-216 also showed significant increases in expression in the cell membrane with cell surface biotinylation. This suggests that longer-term exposure to these three compounds leads to an increase in YFP-hDAT expression in these cells. This also suggests that the long-term increase in uptake seen with salvinorin A by $\mathrm{ASP}^{+}$ uptake may be partially due to increased cell surface expression of DAT. This result also demonstrated that U50,488H and U69,593 have different effects on DAT, and that the novel agonists also display differences among themselves in regulating DAT.

Cell surface biotinylation studies have been used to measure changes in DAT cell surface expression after activation of both D2 and D3 DA receptors (Bolan et al. 2007; Zapata et al. 2007). TIRFM has also successfully been used to measure changes in DAT cell surface expression after exposure to DA and amphetamine (Furman et al. 2009a). As previously mentioned, both of these techniques have limitations. For example, due to the inability of biotin to bind to proteins found within invaginations on the cell membrane, cell surface biotinylation often overestimates the intracellular fraction and underestimates the cell surface fraction. For TIRFM, the major limitation is the size of the evanescent field that only includes proteins that are very close to the cell membrane. This leads to inaccurate measurements of changes in protein cell surface expression 
since proteins moving in and out of the cell membrane that are very close to the membrane will be measured as expressed on the cell surface when they are just below the cell surface.

Overall these data demonstrate that the initial increase in function caused by KOPr agonists is not trafficking-dependent since no changes in cell surface expression were seen after acute exposures, but that there may be increased active DAT found on the cell surface due to activation of inactive DAT already present. After a longer $30 \mathrm{~min}$ incubation with these agonists, U69,593, salvinorin A, and DS-3-216 all caused increased cell surface expression of YFP-hDAT, which may account for some of the increase in $\mathrm{ASP}^{+}$uptake observed at this time point with salvinorin A.

6.1.2.4 KOPr modulates DAT through ERK1/2, is dependent on lipid rafts and does not lead to increased total DAT protein

One of the ways in which increased cell surface expression of proteins can occur is through an increase in total protein within the cell. Therefore we investigated whether the increase in cell surface expression observed at 30 min with U69,593, salvinorin A, and DS-3-216 was due to increased total YFP-hDAT protein within the cell. It was found that after a 30 min incubation, none of the KOPr agonists used in this study caused an increase in total YFP-hDAT protein in HEK-293 cells.

As KOPr agonism leads to activation of ERK1/2 (Belcheva et al. 2005; Kim et al. 2006; McLennan et al. 2008), and ERK1/2 activation causes increased DAT function and cell surface expression (Moron et al. 2003; Bolan et al. 2007; Zapata et al. 2007), the ERK1/2 pathway was investigated to see if this pathway provided a link between KOPr and DAT. It was found that all the agonists activated the ERK1/2 pathway in HEK-293 cells, with a peak occurring at $1 \mathrm{~min}$ for salvinorin $\mathrm{A}$, and $5 \mathrm{~min}$ for the other KOPr agonists. This activation of ERK1/2 by KOPr was inhibited by the MEK1/2 inhibitor U0126, and this inhibition led to attenuation of the functional effect of these compounds on DAT. These data demonstrate that ERK1/2 is a signalling pathway that functions to regulate DAT and that it is modulated by KOPr activation.

As salvinorin A and U69,593 caused increases in cell surface expression after a $30 \mathrm{~min}$ incubation, we next investigated whether inhibition of ERK1/2 could inhibit this increase. Although the increase in cell surface expression by salvinorin A was inhibited by U0126, the changes caused by U69,593 were not significantly inhibited. These data 
suggest that the increase in cell surface expression observed after treatment with these two compounds may be occurring through different pathways.

Both KOPr and DAT are found throughout the cell membrane, including within lipid rafts, which are important regions of cell membranes that are involved in regulation of signalling pathways (Kenworthy et al. 2004). Disruption of lipid rafts by the cholesterol depleter M $\beta C$ inhibited the effect of KOPr activation on DAT. This inhibition could have occurred from: disruption of the physical interaction between KOPr and DAT, interruption of the signalling pathways between the proteins, or changes in confirmation of DAT or KOPr. Further investigation is needed to identify which of these possible scenarios is occurring. 
Table 6.1: Summary of key findings in this study

\begin{tabular}{|c|c|c|c|c|c|}
\hline \multicolumn{6}{|c|}{ KOPr agonists } \\
\hline & U50,488H & U69,593 & Salvinorin A & DS-1-240 & DS-3-216 \\
\hline DAT tissue & $\begin{array}{l}\text { Increased } \\
\text { uptake }\end{array}$ & - & $\begin{array}{l}\text { Increased } \\
\text { uptake }\end{array}$ & $\begin{array}{l}\text { Increased } \\
\text { uptake }\end{array}$ & $\begin{array}{l}\text { Increased } \\
\text { uptake }\end{array}$ \\
\hline $\mathrm{Km} / \mathrm{Vmax}$ & $\begin{array}{l}\text { No change/ } \\
\text { Increased }\end{array}$ & $\begin{array}{l}\text { No change/ } \\
\text { Increased }\end{array}$ & Inconclusive & $\begin{array}{l}\text { No change/ } \\
\text { Increased }\end{array}$ & $\begin{array}{l}\text { No change/ } \\
\text { Increased }\end{array}$ \\
\hline $\begin{array}{l}\text { ASP }{ }^{+} \text {uptake } \\
\text { (acute time) }\end{array}$ & $\begin{array}{l}\text { Increased } \\
\text { uptake }\end{array}$ & $\begin{array}{l}\text { Increased } \\
\text { uptake }\end{array}$ & $\begin{array}{l}\text { Increased } \\
\text { uptake }\end{array}$ & $\begin{array}{l}\text { Increased } \\
\text { uptake }\end{array}$ & $\begin{array}{l}\text { Increased } \\
\text { uptake }\end{array}$ \\
\hline $\begin{array}{l}\text { Nor-BNI and } \\
\text { PTX sensitive } \\
\text { change in } \\
\text { uptake }\end{array}$ & Yes & - & Yes & Yes & Yes \\
\hline $\mathrm{ASP}^{+}$binding & $\begin{array}{l}\text { Non- } \\
\text { significant } \\
\text { increase }\end{array}$ & - & $\begin{array}{l}\text { Non- } \\
\text { significant } \\
\text { increase }\end{array}$ & $\begin{array}{l}\text { Non- } \\
\text { significant } \\
\text { increase }\end{array}$ & $\begin{array}{l}\text { Significant } \\
\text { increase }\end{array}$ \\
\hline $\begin{array}{l}\text { Cell surface } \\
\text { expression } \\
\text { (acute time) }\end{array}$ & No change & No change & No change & No change & No change \\
\hline $\begin{array}{l}\text { ASP }{ }^{+} \text {uptake } \\
(30 \text { min })\end{array}$ & $\begin{array}{l}\text { Increased } \\
\text { uptake }\end{array}$ & - & $\begin{array}{l}\text { Increased } \\
\text { uptake }\end{array}$ & No change & No change \\
\hline $\begin{array}{l}\text { Cell surface } \\
\text { expression } \\
(\mathbf{3 0} \mathrm{min})\end{array}$ & No change & Increased & Increased & No change & Increased \\
\hline ERK1/2 & $\begin{array}{l}\text { Uptake } \\
\text { dependent on } \\
\text { ERK } 1 / 2\end{array}$ & $\begin{array}{l}\text { Uptake } \\
\text { dependent on } \\
\text { ERK } 1 / 2\end{array}$ & $\begin{array}{l}\text { Uptake } \\
\text { dependent on } \\
\text { ERK } 1 / 2\end{array}$ & $\begin{array}{l}\text { Uptake } \\
\text { dependent on } \\
\text { ERK } 1 / 2\end{array}$ & $\begin{array}{l}\text { Uptake } \\
\text { dependent on } \\
\text { ERK1/2 }\end{array}$ \\
\hline $\begin{array}{l}\text { Lipid raft } \\
\text { disruption }\end{array}$ & $\begin{array}{l}\text { Uptake } \\
\text { dependent on } \\
\text { lipid rafts }\end{array}$ & $\begin{array}{l}\text { Uptake } \\
\text { dependent on } \\
\text { lipid rafts }\end{array}$ & $\begin{array}{l}\text { Uptake } \\
\text { dependent on } \\
\text { lipid rafts }\end{array}$ & $\begin{array}{l}\text { Uptake } \\
\text { dependent on } \\
\text { lipid rafts }\end{array}$ & $\begin{array}{l}\text { Uptake } \\
\text { dependent on } \\
\text { lipid rafts }\end{array}$ \\
\hline $\begin{array}{l}\text { Cell surface } \\
\text { expression } \\
\text { and ERK1/2 }\end{array}$ & - & $\begin{array}{l}\text { Independent of } \\
\text { ERK1/2 }\end{array}$ & $\begin{array}{l}\text { Dependent on } \\
\text { ERK } 1 / 2\end{array}$ & - & - \\
\hline SERT tissue & $\begin{array}{l}\text { Decreased } \\
\text { uptake }\end{array}$ & - & $\begin{array}{l}\text { Decreased } \\
\text { uptake }\end{array}$ & $\begin{array}{l}\text { Decreased } \\
\text { uptake }\end{array}$ & - \\
\hline
\end{tabular}




\subsection{Future Directions}

As KOPr increases DAT function after chronic cocaine treatment in the NAc (Thompson et al. 2000), it would be interesting to investigate the effect of the novel compounds on DAT and SERT function after chronic cocaine administration. This could be achieved using RDEV to measure DAT and SERT function in isolated rat brain tissue from rats treated with cocaine and an acute dose of the KOPr agonist. This would give more information about the interaction between drugs of abuse, the transporters, and KOPr activation. This experiment was attempted during this project, and the NAc, striatum, and medial PFC were collected from rats treated once daily with cocaine $(20 \mathrm{mg} / \mathrm{kg})$ for 10 days, followed by a single treatment with a KOPr agonist (treated animals were provided by A. Morani). However, due to the RDEV machine breaking down at this time, the experiment was unable to be completed.

It would also be interesting to investigate the effect of KOPr agonism on cocaine effects on DA binding and transport in a cell model. This could be achieved using ASP $^{+}$ uptake studies, and the binding and uptake of $\mathrm{ASP}^{+}$to cells treated with cocaine could be compared to cells pre-treated with KOPr agonist before cocaine treatment. This would give more mechanistic information on how KOPr agonists inhibit the effects of cocaine on DAT.

To investigate whether KOPr activation leads to increased active DAT at the cell surface, studies can be conducted to investigate changes seen in dimer or oligomer DAT at the cell surface. DAT dimers and oligomers are important for trafficking and function in the cell (Hastrup et al. 2001; Torres et al. 2003) This experiment can be carried out by treating myc-rKOPr- and YFP-hDAT-transfected cells with a KOPr agonist and then chemically cross-linking DAT. Cell surface biotinylation can then be carried out to specifically investigate the DAT protein present at the cell surface. This technique has previously been used to investigate the effect of DA and amphetamine on the production of DAT oligomers from dimer precursors (Chen and Reith 2008). This study would help to elucidate whether this is the method by which acute KOPr agonists increase binding and uptake of $\mathrm{ASP}^{+}$to DAT.

To further investigate the role of lipid rafts in the interaction between KOPr and DAT, it would be useful to carry out co-immunoprecipitation studies with samples treated with or without the lipid raft disrupter $\mathrm{M} \beta \mathrm{C}$. This would confirm whether disrupting lipid 
rafts disrupts the physical interaction between KOPr and DAT or whether changes seen are due to altered signalling pathways. Native PAGE or cross-linking followed by cell surface biotinylation could also be carried out to investigate whether the lipid raft disruption protocol used in this study disrupts DAT oligomers, and this would help elucidate if the effect seen was due to production of active DAT.

It would also be useful to carry out proteomic studies using 2D-difference in gel electrophoresis and mass spectrometry to investigate changes in protein expression after treatment of cells with the KOPr agonists. Changes in protein expression could be compared between the classic and novel KOPr agonists, which may lead to increased information about differences these compounds may have. This could also be carried out in rats treated with the agonists, and could help give more information on side effects by identification of proteins involved in different pathways.

Furthermore, as new novel KOPr agonists and antagonists are synthesised it would be interesting to also carry out screening on these compounds ability to modulate DAT and SERT. It has been previously suggested that mixed action KOPr agonists may be better pharmacotherapies as they would have decreased side effects (Prisinzano et al. 2005). It would therefore be interesting to investigate their effects on DAT and SERT if they show potential in animal models of addiction. 


\subsection{Overall significance}

Overall this research has led to a greater understanding of how KOPr agonists increase DAT function. We found that DAT is regulated by KOPr through the ERK1/2 pathway, and that the rapid increases in uptake of substrate are trafficking-independent and lipid raft-dependent. It also confirms that KOPr agonism regulates SERT function in tissue, causing a decrease in uptake of 5-HT after $15 \mathrm{~min}$ treatment. While there were differences observed between the effect of the classic KOPr agonists and the novel salvinorin-based agonists, these differences were small; therefore, from these data the novel agonists do not appear to regulate DAT in a significantly different way to the classic agonists. However, as these compounds show different side effect profiles in animals (A. Morani, unpublished data), further investigation is warranted to determine the specific mechanisms by which these agonists alter DAT and SERT function. As DAT plays a major role in drug addiction, and SERT plays an important role in depression and stress, this research may help build a picture for a successful pharmacotherapy for drug addiction. 


\section{Appendix 1: Solutions}

\section{Chapter 2 solutions}

$10 \times$ Phosphate Buffered Saline (PBS)

$\begin{array}{lll} & \text { Concentration } & \text { g/L } \\ \mathrm{NaCl} & 1.4 \mathrm{M} & 80 \\ \mathrm{KCl} & 26.8 \mathrm{mM} & 2.0 \\ \mathrm{Na}_{2} \mathrm{HPO}_{4} & 81.0 \mathrm{mM} & 26.8 \\ \mathrm{KH}_{2} \mathrm{PO}_{4} & 14.7 \mathrm{mM} & 2.4 \\ & \mathrm{pH} 7.4 & \end{array}$

LB Agar

g/L

$\mathrm{NaCl}$

10

Yeast Extract

5.0

Bactotryptone

10

Yeast Agar

10

\section{LB Broth}

g/L

$\mathrm{NaCl}$

10

Yeast Extract

5.0

Bactotryptone

10

\section{PBS Ca/Mg}

1 x PBS containing:

$\begin{array}{lll} & \text { Concentration } & \text { g/L } \\ \mathrm{CaCl}_{2} & 0.1 \mathrm{mM} & 0.111 \\ \mathrm{MgCl}_{2} .6 \mathrm{H}_{2} \mathrm{O} & 1 \mathrm{mM} & 0.203 \\ & \mathrm{pH} 8.0 & \end{array}$

PBS Ca/Mg Glycine

PBS $\mathrm{Ca} / \mathrm{Mg}$ containing

Concentration $\quad$ g/L

Glycine

$100 \mathrm{mM}$ 
RIPA buffer

$\begin{array}{lll} & \text { Concentration } & \text { g/L } \\ \text { Tris-HCl } & 10 \mathrm{mM} & 1.2 \\ \mathrm{NaCl} & 150 \mathrm{mM} & 8.766 \\ \text { EDTA } & 1 \mathrm{mM} & 0.37 \\ \text { Triton-X-100 } & 1 \% & 10 \mathrm{~mL} \\ \text { SDS } & 0.1 \% & 1.0 \\ \text { Sodium deoxycholate } & 1 \% & 10 \\ & \mathrm{pH} 7.5 & \end{array}$

\section{$5 \times$ Reducing buffer}

\section{Concentration}

Tris-HCl, $\mathrm{pH} 6.8$

$62.5 \mathrm{mM}$

SDS

$2 \%$

Glycerol

$20 \%$

Bromophenol Blue

$1 \%$

\section{Gels}

Enough for 2 gels

$10 \%$ Separating Gel

$\mathrm{dH}_{2} \mathrm{O}$

$8 \mathrm{~mL}$

1.5 M Tris $\mathrm{pH} 8.8$

$5 \mathrm{~mL}$

$10 \%$ SDS

$200 \mu \mathrm{L}$

Acrylamide (30\% acrylamide/Bis solution

$6.66 \mathrm{~mL}$

$10 \%$ APS

$100 \mu \mathrm{L}$

TEMED

$10 \mu \mathrm{L}$

$100 \%$ isopropanol was layered over the gel while it was setting and was poured off before the stacking gel was added. 
4\% Stacking Gel

$\mathrm{dH}_{2} \mathrm{O}$

$6.1 \mathrm{~mL}$

0.5 M Tris pH 6.8

$2.5 \mathrm{~mL}$

$10 \% \operatorname{SDS}$

$100 \mu \mathrm{L}$

Acrylamide

$1.33 \mathrm{~mL}$

$10 \%$ APS

$50 \mu \mathrm{L}$

TEMED

$10 \mu \mathrm{L}$

$10 \times$ Running buffer

$\begin{array}{lll} & \text { Concentration } & \text { g/L } \\ \text { SDS } & 35 \mathrm{mM} & 10 \\ \text { Tris-HCl } & 250 \mathrm{mM} & 30.3 \\ \text { Glycine } & 1.9 \mathrm{M} & 144.1\end{array}$

Western Transfer Buffer

Concentration g/L

$\begin{array}{lll}\text { Glycine } & 190 \mathrm{mM} & 14.4\end{array}$

$\begin{array}{lll}\text { Tris- } \mathrm{HCl} & 25 \mathrm{mM} & 3.03\end{array}$

Methanol 20\%

$200 \mathrm{~mL}$

$10 \times$ TBS

Concentration g/L

$\begin{array}{lll}\text { Tris } \mathrm{HCl} & 500 \mathrm{mM} & 60.5\end{array}$

$\begin{array}{lll}\mathrm{NaCl} & 1.5 \mathrm{M} & 87.6\end{array}$

$\mathrm{pH} 7.5$

T-TBS

$1 \times$ TBS containing $0.1 \%$ Tween-20 
KREBS Buffer

$\begin{array}{lll} & \text { Concentration } & \text { g/L } \\ \mathrm{NaCl} & 130 \mathrm{mM} & 7.6 \\ \mathrm{KCl} & 1.3 \mathrm{mM} & 0.10 \\ \mathrm{CaCl}_{2} & 2.2 \mathrm{mM} & 0.24 \\ \mathrm{MgSO}_{4} .6 \mathrm{H}_{2} \mathrm{O} & 1.2 \mathrm{mM} & 0.27 \\ \mathrm{KH}_{2} \mathrm{PO}_{4} & 1.2 \mathrm{mM} & 0.16 \\ \mathrm{HEPES} & 10 \mathrm{mM} & 2.6 \\ \mathrm{D}-\mathrm{Glucose} & 10 \mathrm{mM} & 1.8 \\ & \mathrm{pH} 7.4 & \end{array}$

\section{Chapter 3 solutions}

\section{Saline}

$\begin{array}{lll} & \text { Concentration } & \text { g/L } \\ \mathrm{NaCl} & 138 \mathrm{mM} & 8.0 \\ \mathrm{KCl} & 4.1 \mathrm{mM} & 0.31 \\ \mathrm{Na}_{2} \mathrm{HPO}_{4} & 5.1 \mathrm{mM} & 0.80 \\ \mathrm{KH}_{2} \mathrm{PO}_{4} & 5.0 \mathrm{mM} & 0.68 \\ \text { D-glucose } & 2 \% \mathrm{wt} / \mathrm{vol} & 20 \\ & \mathrm{pH} 7.3 & \end{array}$

Krebs, $\mathrm{Na}^{+}$free

Concentration g/L

$\begin{array}{lll}\text { Choline } & 130 \mathrm{mM} & 8.1 \\ \mathrm{KCl} & 1.3 \mathrm{mM} & 0.10 \\ \mathrm{CaCl}_{2} & 2.2 \mathrm{mM} & 0.24 \\ \mathrm{MgSO}_{4} \cdot 6 \mathrm{H}_{2} \mathrm{O} & 1.2 \mathrm{mM} & 0.27 \\ \mathrm{KH}_{2} \mathrm{PO}_{4} & 1.2 \mathrm{mM} & 0.16 \\ \mathrm{HEPES} & 10 \mathrm{mM} & 2.6 \\ \text { D-Glucose } & 10 \mathrm{mM} & 1.8 \\ & \mathrm{pH} 7.4 & \end{array}$


Krebs $\mathrm{Cl}^{-}$free

$\begin{array}{lll} & \text { Concentration } & \text { g/L } \\ \text { Sodium acetate } & 130 \mathrm{mM} & 17.7 \\ \text { Potassium acetate } & 1.3 \mathrm{mM} & 0.13 \\ \text { Calcium acetate } & 2.2 \mathrm{mM} & 0.39 \\ \mathrm{MgSO}_{4} \cdot 6 \mathrm{H}_{2} \mathrm{O} & 1.2 \mathrm{mM} & 0.27 \\ \mathrm{KH}_{2} \mathrm{PO}_{4} & 1.2 \mathrm{mM} & 0.16 \\ \mathrm{HEPES} & 10 \mathrm{mM} & 2.6 \\ \text { D-Glucose } & 10 \mathrm{mM} & 1.8 \\ & \mathrm{pH} 7.4 & \end{array}$

\section{Chapter 5 solutions}

Stripping buffer

$\begin{array}{lll} & \text { Concentration } & \text { g/L } \\ \text { SDS } & 69 \mathrm{mM} & 20 \\ \text { 0.05 M Tris HCl, pH } 6.8 & & 992,5 \mathrm{~mL} \\ \beta \text {-mercaptoethanol } & & 7.5 \mathrm{~mL}\end{array}$




\section{Appendix 2: Chapter 4}

\section{A2.1 $\mathrm{ASP}^{+}$uptake: Time of maximal effect}

Iniital $\mathrm{ASP}^{+}$uptake studies were conducted to investigate when the KOPr agonists had their largest effect within a 5 min period. The uptake of $\mathrm{ASP}^{+}$was compared to uptake before agonist addition as described in Chapter 4. It was found that U50,488H had its maximal effect at 100-160 s after addition (Figure A2.1a), Salvinorin A had its maximal effect 60-120 s after addition (Figure A2.1b), and DS-1-240 and DS-3-216 had their maximal effects at 200-260 s (Figure A2.1c and d). These time points were used for all following studies where the effect of the agonist was measured at the acute time point.

\section{A2.2 Transfection optimisation of $\mathrm{N} 2 \mathrm{~A}$ and $\mathrm{COS7}$ cells}

The co-transfection of HEK-293 cells with myc-KOPr and YFP-hDAT was already established in our laboratory before this research project began. However, cotransfection of N2A and COS-7 cells was not. N2A and COS7 cells are not as easy to transfect as HEK-293 cells so optimisation was carried out to get maximum transfection efficiency. This was carried out using three different protocols:

Protocol One: Cells were transfected using the same protocol as HEK-293 cells Protocol Two: Cell were transfected with the same amount of DNA as HEK-293 cells but with $4 \mu \mathrm{L}$ per dish of Lipofectamine 2000 rather than $3 \mu \mathrm{L}$.

Protocol Three: Cells were transfected with double the plasmid DNA of HEK-293 cells and $4 \mu \mathrm{L}$ of Lipofectamine 2000.

Transfected cells were looked at after $48 \mathrm{~h}$ using confocal microscopy and the protocol with the highest number of YFP-hDAT cells for each cell line were used for future experiments (Figure A2.2). It was found that Protocol 3 produced the highest transfection rates in both $\mathrm{N} 2 \mathrm{~A}$ and COS7 cells. 

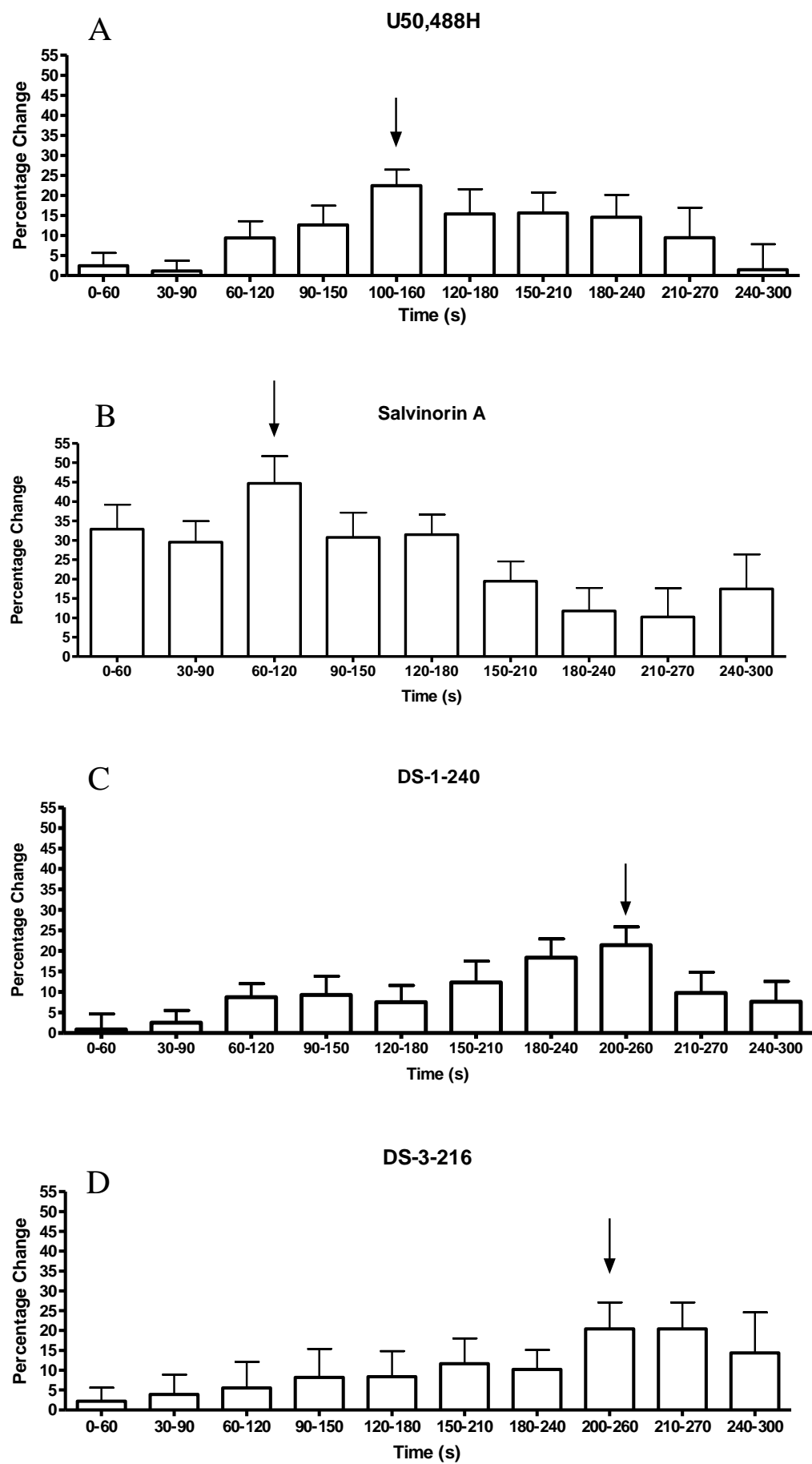

Figure A2.1 Time of maximal effect of KOPr agonists on $\mathrm{ASP}^{+}$uptake. Initial experiments were carried out in HEK-293 cells transfected with myc-rKOPr and YFPhDAT to find the time of maximal effect of the KOPr agonists on $\mathrm{ASP}^{+}$uptake by YFPhDAT. (A) U50,588H showed its maximal effect at 100-160 s, (B) salvinorin A showed its maximal effect at 60-120 s, (C,D) DS-1-240 and DS-3-216 showed their maximal effect at 200-260 s. $\mathrm{n}=46-60$ cells, a minimum of 2 dishes over 3 transfections. 


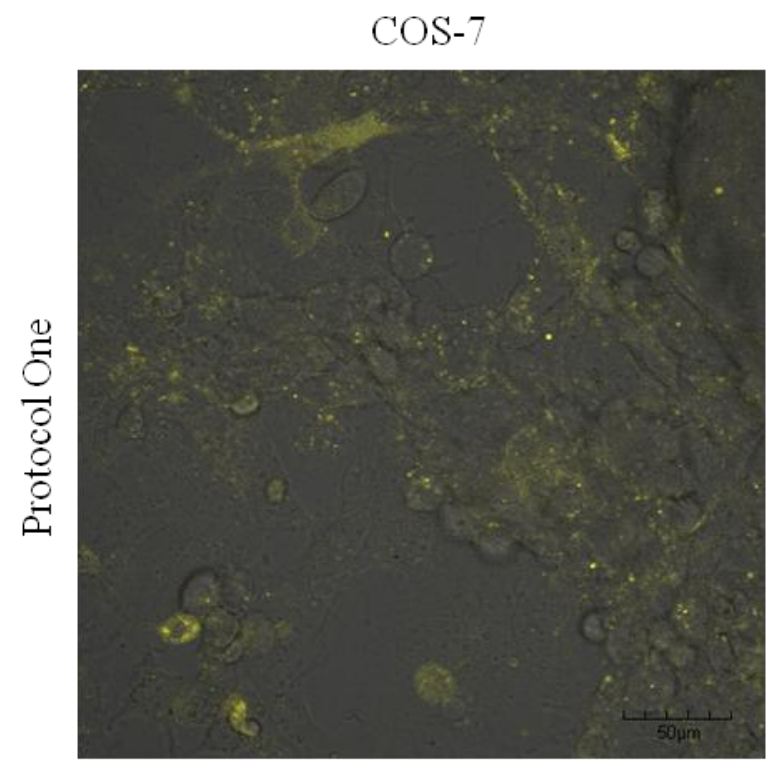

N2A
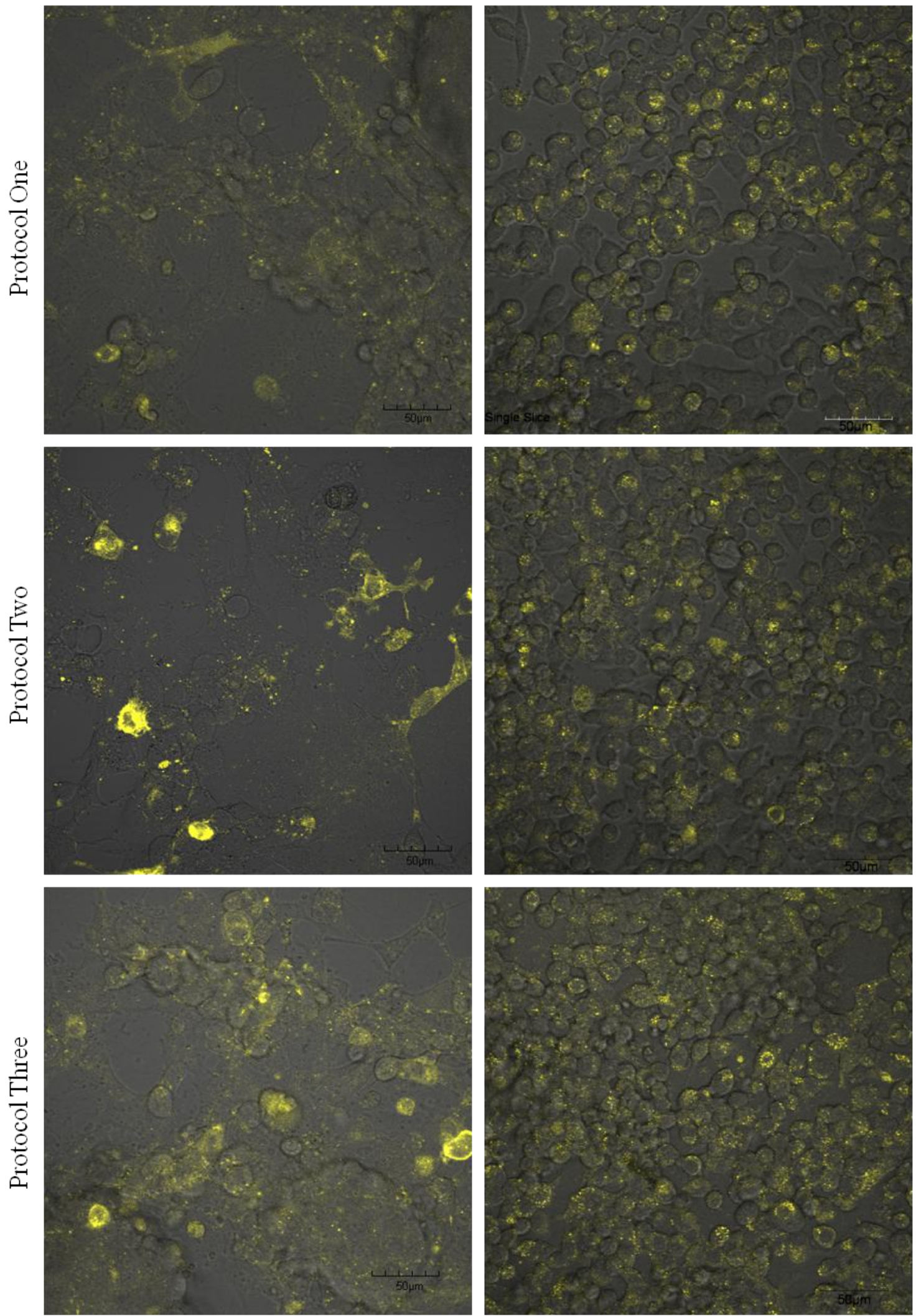

Figure A2.2 N2A and COS7 cells transfected with YFP-hDAT and myc-rKOPr with three different protocols. N2A cells and COS7 cells were transfected using three different protocols and then imaged to determine which protocol gave the highest transfection rates. For both cell lines protocol three gave the highest number of transfected cells and this protocol was used for all further studies in these cell lines. 


\section{A2.3 Setting up TIRFM}

Initial TIRFM experiments were carried out to set up the technique in our laboratory following a previous study in which they found $10 \mu \mathrm{M}$ DA led to an immediate increase in YFP-hDAT cell surface expression (Furman et al. 2009a). Initial experiments carried out showed that in our system with our experimental set up we were able to measure this increase accurately with an increase of 10-15\% after $120 \mathrm{~s}$. An increase of between 10-15\% was observed by Furman et al. (Furman et al. 2009a). This demonstrates that our TIRFM system was working correctly (Figure A2.3). Initial experiments also found that cells needed to be pre-incubated in the chamber for $20 \mathrm{~min}$ before experiments took place to minimise drift and that for 40 min studies the laser needed to be turned on and off between each image to prevent photobleaching. It was also found that cells were healthier if they were kept in their growth media rather than in KREBs buffer, and saturated with carbogen.

During these experiments we also found the best exposure time vs. laser intensity to use. With our cells we found that a long exposure time $(3 \mathrm{~s})$ with a low laser power $(0.1-0.3 \%)$ prevented photobleaching successfully and therefore we used this long exposure time for all future experiments. 

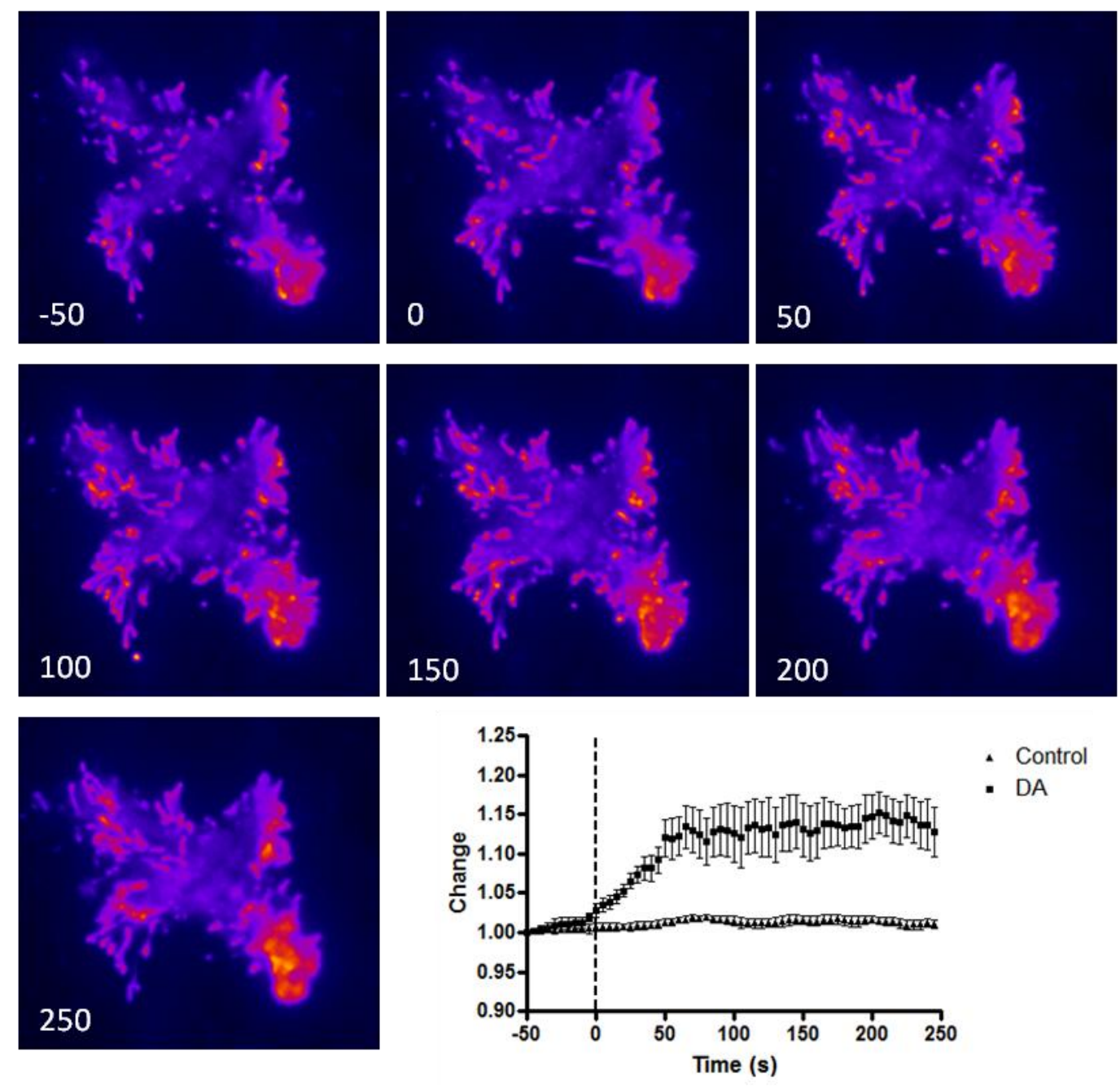

Figure A2.3 DA causes increased YFP-hDAT cell surface expression. Top panels: Incubation of HEK-293 cells with DA for $250 \mathrm{~s}$ caused an increase in YFP-hDAT cell surface expression as measured by TIRFM. Images were taken before DA addition ( -50 $\mathrm{s})$, at DA addition (0 s), and after DA addition (50, 100, 150, 200, $250 \mathrm{~s})$. Graphical analysis indicates a rapid increase of YFP-hDAT, which plateaued after $70 \mathrm{~s}$. The graph represents the average, \pm SEM for 6 cells in both control and DA treated groups. 


\section{Appendix 3: Chapter 5}

\section{A3.1 ERK1/2 optimisation}

\section{A.3.1 Serum starvation time}

A protocol was used to measure ERK1/2 activation without serum, as serum causes basal ERK1/2 activation. There are varying times of serum starvation published, with times between 30 min and $24 \mathrm{~h}$ (Belcheva et al. 2005; Bolan et al. 2007; Groer et al. 2007; McLennan et al. 2008). Initial experiments were carried out to find out which time was the best for our protocol. HEK-293 cells started to look sick at the $16 \mathrm{~h}$ time point, and therefore Western blot analysis of basal ERK1/2 levels of the cells after 30 min and $4 \mathrm{~h}$ serum starvation was performed. As there was no real difference between basal ERK1/2 phosphorylation between these time points (Figure A3.1) and due to the timing of the experiment serum starving for $30 \mathrm{~min}$ was carried out for all future experiments.

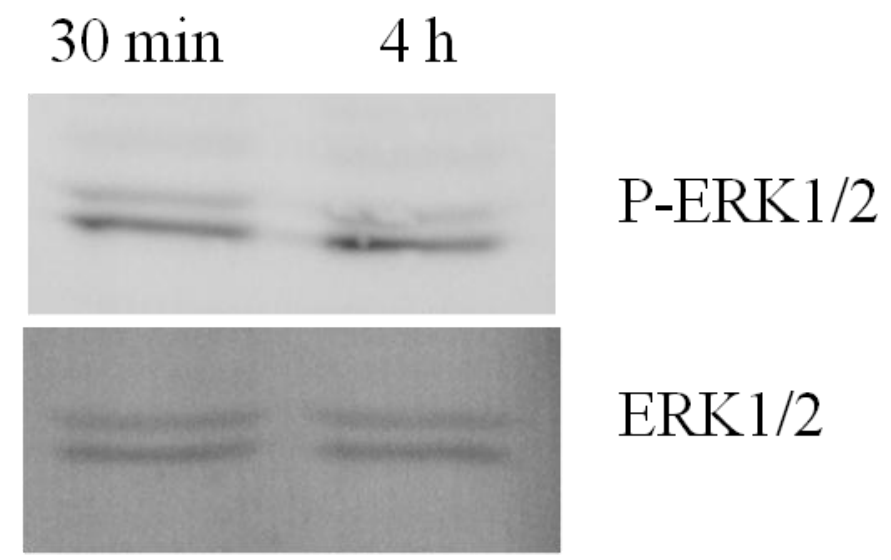

Figure A3.1: Comparison between basal P-ERK1/2 levels after serum starvation for $30 \mathrm{~min}$ (left band) and $4 \mathrm{~h}$ (right band). Cells were serum starved for 30 min or 4 $\mathrm{h}$ and western blotting was carried out for P-ERK1/2 and ERK1/2. There was no real difference between basal P-ERK1/2 between either treatment and therefore a $30 \mathrm{~min}$ serum starvation protocol was carried out for future experiments. 
A3.2.2 Incubation time with P-ERK1/2 antibody

Two different incubation times were tested with the P-ERK1/2 antibody, $1 \mathrm{~h}$ at room temperature, and overnight $(20 \mathrm{~h})$ at $4^{\circ} \mathrm{C}$. As there was increased signal with the overnight incubation this time was used for all further experiments (Figure A3.2). A $1 \mathrm{~h}$ incubation at room temperature with the ERK1/2 antibody produced a strong signal and therefore no other times were tested.

Control UV

\section{$20 \mathrm{~h} \mathrm{P-ERK} 1 / 2$}

\section{$1 \mathrm{~h}$ P-ERK1/2}

Figure A3.2: Comparison between a $1 \mathrm{~h}$ incubation with P-ERK1/2 antibody and a $20 \mathrm{~h}$ incubation. Cells were serum starved for $30 \mathrm{~min}$ and then either left in the incubator for $1 \mathrm{~h}$ or UVed for $1 \mathrm{~h}$. Cells were then lysed and Western blot analysis was carried out for P-ERK1/2. A $1 \mathrm{~h}$ incubation at room temperature and a $20 \mathrm{~h}$ incubation at $4^{\circ} \mathrm{C}$ was carried out with the P-ERK1/2 primary antibody and results compared. As shown, a $20 \mathrm{~h}$ incubation iwht the antibody gave stronger results than the $1 \mathrm{~h}$ and was therefore used for future experiments. 


\section{$\underline{\text { A3.2.3 Protein amount }}$}

The amount of protein loaded on the gel was also optimised to get the best P-ERK1/2 bands. Cells were treated with salvinorin A and Western blotting was conducted as described in chapter 5 . Initially $25 \mu \mathrm{g}$ of protein was loaded onto the gel, but only the UV positive control was visible, therefore, the protein concentration was doubled to 50 $\mu \mathrm{g}$ and the results were much clearer (Figure A3.3).

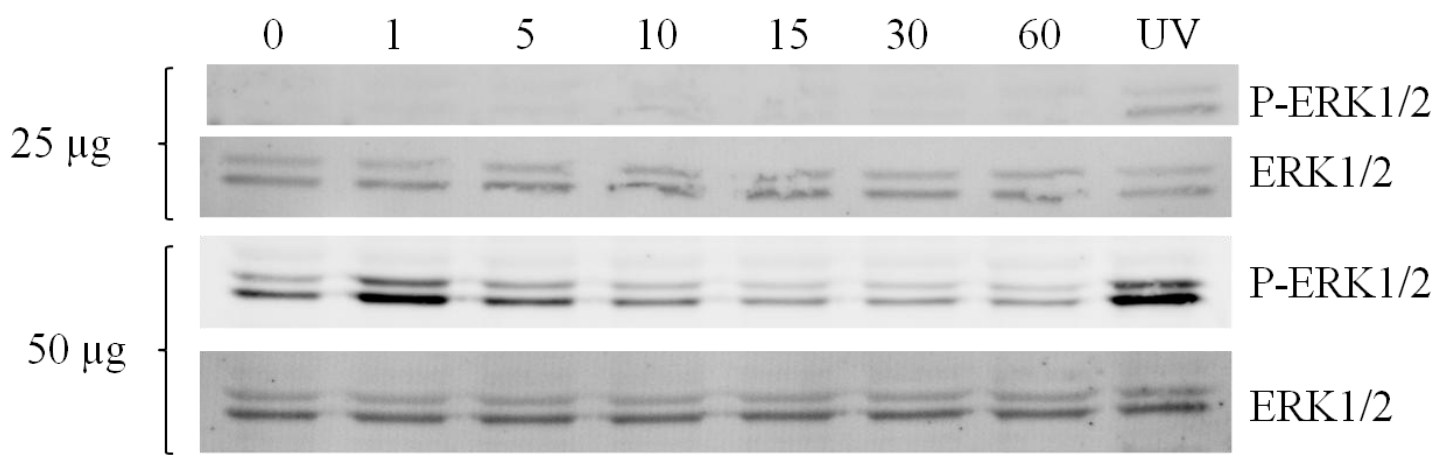

Figure A3.3: Comparison between $25 \mu \mathrm{g}$ of protein lysate and $50 \mu \mathrm{g}$ of protein lysate. As shown $50 \mu \mathrm{g}$ of protein gave stronger results compared to $25 \mu \mathrm{g}$ of protein after being treated with salvinorin A. Therefore $50 \mu \mathrm{g}$ of protein was used for all future experiments. 


\section{References}

A.P.A. 2000. Diagnostic and statistical manual of mental disorders: DSM-IV-TR, 4th ed. American Psychiatric Association, Washington, DC.

Acri, J.B., Thompson, A.C., and Shippenberg, T. 2001. Modulation of pre- and postsynaptic dopamine D2 receptor function by the selective kappa-opioid receptor agonist U69593. Synapse 39: 343-350.

Adinoff, B. 2004. Neurobiologic processes in drug reward and addiction. Harvard Review of Psychiatry 12: 305-320.

Adkins, E.M., Samuvel, D.J., Fog, J.U., Eriksen, J., Jayanthi, L.D., Vaegter, C.B., Ramamoorthy, S., and Gether, U. 2007. Membrane mobility and microdomain association of the dopamine transporter studied with fluorescence correlation spectroscopy and fluorescence recovery after photobleaching. Biochemistry 46: 10484-10497.

al Qatari, M., Khan, S., Harris, B., and Littleton, J. 2001. Acamprosate is neuroprotective agaiinst glutamate-induced excitotoxicity when enhanced by ethanol withdrawal in neocortical cultures of fetal rat brain. Alcoholism: Clinical and Experimental Research 25: 1276-1283.

Albertson, D.N., and Grubbs, L.E. 2009. Subjective effects of salvia divinorum: LSDor marijuana-like? Journal of Psychoactive Drugs 41: 213-217.

Alex, K.D., and Pehek, E.A. 2007. Pharmacological mechanisms of serotonergic regulation of dopamine neurotransmission. Pharmacology \& Therapeutics 113: 296-320.

Ansonoff, M.A., Zhang, J.W., Czyzyk, T., Rothman, R.B., Stewart, J., Xu, H., Zjwiony, J., Siebert, D.J., Yang, F., Roth, B.L., et al. 2006. Antinociceptive and hypothermic effects of salvinorin A are abolished in a novel strain of kappaopioid receptor-1 knockout mice. Journal of Pharmacology and Experimental Therapeutics 318: 641-648.

Assenio, V.J., Miralles, A., and Garcia-Sevilla, J.A. 2006. Stimulation of mitogenactivated protein kinase kinase $(\mathrm{MEK} 1 / 2)$ by mu-, delta- and kappa-opioid receptor agonists in the rat brain: regulation by chronic morphine and opioid withdrawal. European Journal of Pharmacology 539: 49-56.

Avidorreiss, T., Zippel, R., Levy, R., Saya, D., Ezra, V., Barg, J., Matusleibovitch, N., and Vogel, Z. 1995. Kappa-opioid receptor-transfected cell-lines - modulation of adenylyl-cyclase activity following acute and chronic opioid treatments. Febs Letters 361: 70-74.

Axelrod, D. 2001. Total internal reflection fluorescence microscopy in cell biology. Traffic 2: 764-774.

Bach, A.W.J., Lan, N.C., Johnson, D.L., Abell, C.W., Bembenek, M.E., Kwan, S.-W., Seeburg, P.H., and Shih, J.C. 1988. cDNA cloning of human liver monoamine oxidase A and B: molecular basis of differences in enzymatic properties. Proceedings of the National Academy of Sciences of the United States of America 85: 4934-4938.

Baier, C.J., Gallegos, C.E., Levi, V., and Barrantes, F.J. 2010. Cholesterol modulation of nicotinic acetylcholine receptor surface mobility. European Biophysics Journal 39: 213-227.

Baker, L.E., Panos, J.J., Killinger, B.A., Peet, M.M., Bell, L.M., Haliw, L.A., and Walker, S.L. 2009. Comparison of the discriminative stimulus effects of salvinorin A and its derivatives to U69,593 and U50,488 in rats. Psychopharmacology 203: 203-211.

Balfour, D., Benowitz, N., Fagerstrom, K., Kunze, M., and Keil, U. 2000. Diagnosis and treatment of nicotine dependence with emphasis on nicotine replacement therapy. European Heart Journal 21: 438-445. 
Batchelor, M., and Schenk, J.O. 1998. Protein kinase A activity may kinetically upregulate the striatal transporter for dopamine. Journal of Neuroscience 18: 10304-10309.

Beardsley, P.M., Howard, J.L., Shelton, K.L., and Carroll, F.I. 2005. Differential effects of the novel kappa opioid receptor antagonist, JDTic, on reinstatement of cocaine-seeking induced by footshock stressors vs cocaine primes and its antidepressant-like effects in rats. Psychopharmacology 183: 118-126.

Beerepoot, P., Lam, V., Luu, A., and Tsoi, B. 2008. Effects of salvinorin A on locomotor sensitization to D2/D3 dopamine agonist quinpirole. Neuroscience Letters 446: 101-104.

Beguin, C., Duncan, K.K., Munro, T.A., Ho, D.M., Xu, W., Liu-Chen, L.Y., Carlezon, W.A., and Cohen, B. 2009. Modification of the furan ring of salvinorin A: identification of a selective partial agonist at the kappa opioid receptor. Bioorganic \& Medicinal Chemistry 17: 1370-1380.

Beguin, C., Potter, D.N., DiNieri, J.A., Munro, T.A., Richards, M.R., Paine, T.A., Berry, L., Zhao, Z., Roth, B.L., Xu, W., et al. 2008. N-methylacetamide analog of salvinorin A: a highly potent and selective kappa-opioid receptor agonist with oral efficacy. Journal of Pharmacology and Experimental Therapeutics 324: 188-195.

Beguin, C., Richards, M.R., Li, J.G., Wang, Y.L., Xu, W., Liu-Chen, L.Y., Carlezon, W.A., and Cohen, B.M. 2006. Synthesis and in vitro evaluation of salvinorin A analogues: effect of configuration at $\mathrm{C}(2)$ and substitution at $\mathrm{C}(18)$. Bioorganic \& Medicinal Chemistry Letters 16: 4679-4685.

Beguin, C., Richards, M.R., Wang, Y.L., Chen, Y., Liu-Chen, L.Y., Ma, Z.Z., Lee, D.Y.W., Carlezon, W.A., and Cohen, B.M. 2005. Synthesis and in vitro pharmacological evaluation of salvinorin A analogues modified at $\mathrm{C}(2)$. Bioorganic \& Medicinal Chemistry Letters 15: 2761-2765.

Belcheva, M.M., Clark, A.L., Haas, P.D., Serna, J.S., Hahn, J.W., Kiss, A., and Coscia, C.J. 2005. $\mu$ and $\kappa$ opioid receptor activate ERK/MAPK via different protein kinase $\mathrm{C}$ isoforms and secondary messengers in astrocytes. The Journal of Biological Chemistry 280: 27662-27669.

Bengel, D., Murphy, D.L., Andrews, A.M., Wichems, C.H., Feltner, D., Heils, A., Mossner, R., Westphal, H., and Lesch, K.-P. 1998. Altered brain serotonin homeostasis and locomotor insensitivity to 3,4methylenedioxymethamphetamine ('"ecstacy") in serotonin transporter-deficient mice. Molecular Pharmacology 53: 649-655.

Berger, B., Rothmaier, A.K., Wedekind, F., Zentner, J., Feuerstein, T.J., and Jackisch, R. 2006. Presynaptic opioid receptors on noradrenergic and serotonergic neurons in the human as compared to the rat neocortex. British Journal of Phamacology 148: $795-806$.

Berridge, K.C., and Robinson, T.E. 1995. The mind of an addicted brain: neural sensitization of wanting versus liking. Current Directions in Psychological Science 4: 71-76.

Beuming, T., Shi, L., Javitch, J.A., and Weinstein, H. 2006. A comprehensive structurebased alignment of prokaryotic and eukaryotic neurotransmitter $/ \mathrm{Na}^{+}$symporters (NSS) aids in the use of the LeuT structure to probe NSS structure and function. Molecular Pharmacology 70: 1630-1642.

Beuten, J., Payne, T.J., Ma, J.Z., and Li, M.D. 2006. Significant association of catecholO-methyltransferase (COMT) haplotypes with nicotine dependence in male and female smokers of two ethnic populations. Neuropsychopharmacology 31: 675684. 
Bikbulatov, R.V., Stewart, J., Jin, W., Yan, F., Roth, B.L., Ferreira, D., and Zjawiony, J.K. 2008. Short synthesis of a novel class of salvinorin A analogs with hemiacetalic structure. Tetrahedron Letters 49: 937-940.

Bikbulatov, R.V., Yan, F., Roth, B.L., and Zjawiony, J.K. 2007. Convenient synthesis and in vitro pharmacological activity of 2-thioanalogs of salvinorins $\mathrm{A}$ and $\mathrm{B}$. Bioorganic and Medicinal Chemistry Letters 17: 2229-2232.

Binda, F., Dipace, C., Bowton, E., Robertson, S.D., Lute, B.J., Fog, J.U., Zhang, M., Sen, N., Colbran, R.J., Gnegy, M., et al. 2008. Syntaxin 1A interaction with the dopamine transporter promotes amphetamine-induced dopamine efflux. Molecular Pharmacology 74: 1101-1108.

Bjorklund, N.L., Volz, T.J., and Schenk, J.O. 2007. Differential effects of $\mathrm{Zn}^{2+}$ on the kinetics and cocaine inhibition of dopamine transport by the human and rat dopamine transporters. European Journal of Pharmacology 565: 17-25.

Blakely, R.D., Berson, H.E., Fremeau, R.T.J., Caron, M.G., Peek, M.M., Prince, H.K., and Bradley, C.C. 1991. Cloning and expression of a functional serotonin transporter from rat brain. Nature 354: 66-70.

Blum, K., Noble, E.P., Sheridan, P.J., Montgomery, A., Ritchie, T.L., Ozkaragoz, T., Fitch, R.J., Wood, R.C., Finley, O., and Sadlack, F. 1993. Genetic predisposition in alcoholism: association of the $\mathrm{D}_{2}$ dopamine receptor TaqI B1 RFLP with severe alcoholics. Alcohol 10: 59-67.

Boden, J.M., Fergusson, D.M., and Horward, J. 2006. Illicit drug use and dependence in a new zealand birth cohort. Australian \& New Zealand Journal of Psychiatry 40: 156-163.

Boja, J.W., and Kuhar, M.J. 1989. $\left[{ }^{3} \mathrm{H}\right]$ cocaine binding and inhibition of $\left[{ }^{3} \mathrm{H}\right]$ dopamine uptake is similar in both the rat striatum and nucleus accumbens. European Journal of Pharmacology 173: 215-217.

Bolan, E.A., Kivell, B., Jaligam, V., Oz, M., Jayanthi, L.D., Han, Y., Sen, N., Urizar, E., Gomes, I., Devi, L.A., et al. 2007. D2 receptors regulate dopamine transporter function via an extracellular signal-regulated kinases 1 and 2dependent and phosphoinositide 3 kinase-independent mechanism. Molecular Pharmacology 71: 1222-1232.

Braida, D., Capurro, V., Zani, A., Rubino, T., Vigano, D., Parolaro, D., and Sala, M. 2009. Potential anxiolytic- and antidepressant-like effects of salvinorin A, the main active ingredient of Salvia divinorum, in rodents. British Journal of Pharmacology 157: 844-853.

Braida, D., Limonta, V., Capurro, V., Fadda, P., Rubino, T., Mascia, P., Zani, A., Gori, E., Fratta, W., Parolaro, D., et al. 2008. Involvement of kappa-opioid and endocannabinoid system on salvinorin A-induced reward. Biological Psychiatry 63: $286-292$.

Braida, D., Limonta, V., Pegorini, S., Zani, A., Guerini-Rocco, C., Gori, E., and Sala, M. 2007. Hallucinatory and rewarding effect of salvinorin A in zebrafish: kappaopioid and CB1-cannabinoid receptor involvement. Psychopharmacology 190: 441-448.

Brasser, S.M., McCaul, M.E., and Houtsmuller, E.J. 2004. Alcohol effects during acamprosate treatment: a dose-response study in humans. Alcoholism: Clinical and Experimental Research 28: 1074-1083.

Braye, K., Harwood, T., Inder, R., Beasley, R., and Robinson, G. 2007. Poppy seed tea and opiate abuse in new zealand. Drug and Alcohol Review 26: 215-219.

Brodie, M.S., Pesold, C., and Appel, S.B. 1999. Ethanol directly excites dopaminergic ventral tegmental area reward neurons. Alcoholism: Clinical and Experimental Research 23: 1848-1852. 
Bruchas, M.R., and Chavkin, C. 2010. Kinase cascades and ligand-directed signaling at the kappa opioid receptor. Psychopharmacology 210: 137-147.

Bruchas, M.R., Land, B.B., Aita, M., Xu, M., Barot, S.K., Li, S., and Chavkin, C. 2007a. Stress-induced p38 mitogen-activated protein kinase activation mediates א-opioid-dependent dysphoria. The Journal of Neuroscience 27: 11614-11623.

Bruchas, M.R., Land, B.B., and Chavkin, C. 2010. The dynorphin/kappa opioid system as a modulator of stress-induced and pro-addictive behaviors. Brain Research 1314: 44-55.

Bruchas, M.R., Macey, T.A., Lowe, J.D., and Chavkin, C. 2006. Kappa opioid receptor activation of p38 MAPK is GRK3- and arrestin-dependent in neurons and astrocytes. Journal of Biological Chemistry 281: 18081-18089.

Bruchas, M.R., Xu, M., and Chavkin, C. 2008. Repeated swim stress induces kappa opioid-mediated activation of extracellular signal-regulated kinase 1/2. Neuroreport 19: 1417-1422.

Bruchas, M.R., Yang, T., Schreiber, S., DeFino, M., Kwan, S.C., Li, S., and Chavkin, C. 2007b. Long-acting kappa opioid antagonists disrupt receptor signaling and produce noncompetitive effects by activating c-Jun N-terminal kinase. Journal of Biological Chemistry 282: 29803-29811.

Budygin, E.A., Phillips, P.E.M., Robinson, D.L., Kennedy, A.P., Gainetdinov, R.R., and Wightman, R.M. 2001. Effect of acute ethanol on striatal dopamine neurotransmission in ambulatory rats. The Journal of Pharmacology and Experimental Therapeutics 297: 27-34.

Burgess, K.S.D., Kable, J.W., and Justice, J.B. 1999. Rotating disk electrode voltammetry applied to the kinetics of uptake and efflux in wild-type and mutant catecholamine transporters. Electroanalysis 11: 337-343.

Burmeister, J.J., Lungren, E.M., and Neisewander, J.L. 2003. Effects of fluoxetine and $d$-fenfluramine on cocaine-seeking behavior in rats. Psychopharmacology 168: 146-154.

Burnette, W.B., Bailey, M.D., Kukoyi, S., Blakely, R.D., Trowbridge, C.G., and Justice, J.B. 1996. Human norepinephrine transporter kinetics using rotating disk electrode voltammetry. Analytical Chemistry 68: 2932-2938.

Butelman, E.R., Harris, T.J., and Kreek, M.J. 2004. The plant-derived hallucinogen, salvinorin A, produces kappa-opioid agonist-like discriminative effects in rhesus monkeys. Psychopharmacology 172: 220-224.

Butelman, E.R., Prisinzano, T.E., Deng, H., Rus, S., and Kreek, M.J. 2009. Unconditioned behavioral effects of the powerful $\kappa$-opioid hallucinogen, salvinorin A: fast onset and entry into cerebrospinal fluid. Journal of Pharmacology and Experimental Therapeutics 328: 588-597.

Butelman, E.R., Rus, S., Prisinzano, T.E., and Kreek, M.J. 2010. The discriminative effects of the $\kappa$-opioid hallucinogen salvinorin A in nonhuman primates: dissociation from classic hallucinogen effects. Psychopharmacology 210: 253262.

Capasso, R., Borrelli, F., Capasso, F., Siebert, D.J., Stewart, D.J., Zjawiony, J.K., and Izzo, A.A. 2006. The hallucinogenic herb Salvia divinorum and its active ingredient salvinorin A inhibit enteric cholinergic transmission in the guinea-pig ileum. Neurogastroenterol. Motil. 18: 69-75.

Capasso, R., Borrelli, F., Cascio, M., Aviello, G., Huben, K., Zjawiony, J., Marini, P., Romano, B., Di Marzo, V., Capasso, F., et al. 2008a. Inhibitory effect of salvinorin A, from Salvia divinorum, on ileitis-induced hypermotility: cross-talk between K-opioid and cannabinoid CB1 receptors. British Journal of Pharmacology 155: 681-689. 
Capasso, R., Borrelli, F., Zjawiony, J., Kutrzeba, L., Aviello, G., Sarnelli, G., Capasso, F., and Izzo, A.A. 2008b. The hallucinogenic herb Salvia divinorum and its active ingredient salvinorin A reduce inflammation-induced hypermotility in mice. Neurogastroenterol. Motil. 20: 142-148.

Carey, A.N., Borozny, K., Aldrich, J.V., and McLaughlin, J.P. 2007. Reinstatement of cocaine place-conditioning prevented by the peptide kappa-opioid receptor antagonist arodyn. European Journal of Pharmacology 569: 84-89.

Carlezon, W.A., Beguin, C., DiNieri, J.A., Baumann, M.H., Richards, M.R., Todtenkopf, M.S., Rothman, R.B., Ma, Z.Z., Lee, D.Y.W., and Cohen, B.M. 2006. Depressive-like effects of the kappa-opioid receptor agonist salvinorin A on behavior and neurochemistry in rats. Journal of Pharmacology and Experimental Therapeutics 316: 440-447.

Carlezon, W.A.J., Beguin, C., Knoll, A.T., and Cohen, B.M. 2009. Kappa-opioid ligands in the study and treatment of mood disorders. Pharmacology \& Therapeutics 123: 334-343.

Carroll, F.I., Blough, B.E., Mascarella, S.W., Navarro, H.A., Eaton, J.B., Lukas, R.J., and Damaj, M.I. 2010. Synthesis and biological evaluation of bupropion analogues as potential pharmacotherapies for smoking cessation. Journal of Medicinal Chemistry 11: 2204-2214.

Carroll, F.I., Thomas, J.B., Dykstra, L.A., Granger, A.L., Allen, R.M., Howard, J.L., Pollard, G.T., Aceto, M.D., and Harris, L.S. 2004. Pharmacological properties of JDTic: a novel $\kappa$-opioid receptor antagonist. European Journal of Pharmacology 501: 111-119.

Chang, A.S., and Lam, D.M.-K. 1998. Mechanistic analyses of ion dependences in a high-affinity human serotonin transporter system in transfected murine fibroblast cells. Journal of Physiology 501: 903-913.

Chartoff, E.H., Potter, D., Damez-Werno, D., Cohen, B.M., and Carlezon Jr, W.A. 2008. Exposure to the selective kappa-opioid receptor agonist salvinorin A modulates the behavioral and molecular effects of cocaine in rats. Neuropsychopharmacology 33: 2676-2687.

Chavkin, C., Sud, S., Jin, W.Z., Stewart, J., Zjawiony, J.K., Siebert, D.J., Toth, B.A., Hufeisen, S.J., and Roth, B.L. 2004. Salvinorin A, an active component of the hallucinogenic sage Salvia divinorum is a highly efficacious kappa-opioid receptor agonist: structural and functional considerations. Journal of Pharmacology and Experimental Therapeutics 308: 1197-1203.

Chefer, V., Thompson, A.C., and Shippenberg, T.S. 1999. Modulation of cocaineinduced sensitization by kappa-opioid receptor agonists - role of the nucleus accumbens and medial prefrontal cortex. Annals New York Academy of Sciences 877: 803-806.

Chefer, V.I., Morón, J.A., Hope, B., Rea, W., and Shippenberg, T.S. 2000. Kappaopioid receptor activation prevents alterations in mesocortical dopamine neurotransmission that occur during abstinence from cocaine. Neuroscience 101: 619-627.

Chefer, V.I., and Shippenberg, T.S. 2002. Changes in basal and cocaine-evoked extracellular dopamine uptake and release in the rat nucleus accumbens during early abstinence from cocaine: quantitative determination under transient conditions. Neuroscience 112: 907-919.

Chen, N., and Reith, M.E.A. 2008. Substrates dissociate dopamine transporter oligomers. Journal of Neurochemistry 105: 910-920.

Chen, N., Trowbridge, C.G., and Justice, J.B. 1999a. Cationic modulation of human dopamine transporter: dopamine uptake and inhibition of uptake. The Journal of Pharmacology and Experimentla Therapeutics 290: 940-949. 
Chen, N.H., and Justice, J.B. 2000. Differential effect of structural modification of human dopamine transporter on the inward and outward transport of dopamine. Molecular Brain Research 75: 208-215.

Chen, N.H., Trowbridge, C.G., and Justice, J.B. 1999b. Cationic modulation of human dopamine transporter: dopamine uptake and inhibition of uptake. Journal of Pharmacology and Experimental Therapeutics 290: 940-949.

Chen, Y., Chen, C.G., Wang, Y.L., and Liu-Chen, L.Y. 2006. Ligands regulate cell surface level of the human kappa opioid receptor by activation-induced downregulation and pharmacological chaperone-mediated enhancement: differential effects of nonpeptide and peptide agonists. Journal of Pharmacology and Experimental Therapeutics 319: 765-775.

Chen, Y., Wang, Y., Ji, W., Xu, P., and Xu, T. 2008. A pre-docking role for microtubules in insulin-stimulated glucose transporter 4 translocation. FEBS Journal 275: 705-712.

Chi, L.M., and Reith, M.E.A. 2003. Substrate-induced trafficking of the dopamine transporter in heterologously expressing cells and in rat striatal synaptosomal preparations. Journal of Pharmacology and Experimental Therapeutics 307: 729-736.

Ciliax, B.J., Heilman, C., Demchyshyn, L.L., Pristupa, Z.B., Ince, E., Hersch, S.M., Niznik, H.B., and Levey, A.I. 1995. The dopamine transporter: immunochemical characterization and localization in brain. The Journal of Neuroscience 15: 1714-1723.

Clayton, C.C., Xu, M., and Chavkin, C. 2009. Tyrosine phosphorylation of $\mathrm{K}_{\mathrm{ir}} 3$ following $\kappa$-opioid receptor activation of p38 MAPK causes heterologous desensitization. Journal of Biological Chemistry 284: 31872-31881.

Coe, J.W., Brooks, P.R., Vetelino, M.G., Wirtz, M.C., Arnold, E.P., Huang, J., Sands, S.B., Davis, T.I., Lebel, L.A., Fox, C.B., et al. 2005. Varenicline: An $\alpha 4 \beta 2$ nicotine receptor partial agonist for smoking cessation. Journal of Medicinal Chemistry 48: 3474-3477.

Cohen, B.M., and Murphy, B. 2008. The effects of pentazocine, a kappa agonist, in patients with mania. International Journal of Neuropsychopharmacology 11: 243-247.

Collins, S.L., D'Addario, C., and Izenwasser, S. 2001a. Effects of kappa-opioid receptor agonists on long-term cocaine use and dopamine neurotransmission. European Journal of Pharmacology 426: 25-34.

Collins, S.L., Gerdes, R.M., D'Addario, C., and Izenwasser, S. 2001b. kappa opioid agonists alter dopamine markers and cocaine-stimulated locomotor activity. Behavioural Pharmacology 12: 237-245.

Collins, S.L., Kunko, P.M., Ladenheim, B., Cadet, J.L., Carroll, F.I., and Izenwasser, S. 2002. Chronic cocaine increases kappa-opioid receptor density: lack of effect by selective dopamine uptake inhibitors. Synapse 45: 153-158.

Copeland, B.J., Vogelsberg, V., Neff, N.H., and Hadjiconstantinou, M. 1996. Protein kinase $\mathrm{C}$ activators decrease dopamine uptake into striatal synaptosomes. The Journal of Pharmacology and Experimental Therapeutics 277: 1527-1532.

Corkery, J.M., Schifano, F., Ghodse, A.H., and Oyefeso, A. 2004. The effects of methadone and its role in fatalities. Human Psychopharmacology-Clinical and Experimental 19: 565-576.

Crits-Christoph, P., Newberg, A., Wintering, N., Ploessl, K., Gibbons, M.B.C., RingKurtz, S., Gallop, R., and Present, J. 2008. Dopamine transporter levels in cocaine dependent subjects. Drug and Alcohol Dependence 98: 70-76. 
Czoty, P.W., Ginsburg, B.C., and Howell, L.L. 2002. Serotonergic attenuation of the reinforcing and neurochemical effects of cocaine in squirrel monkeys. Journal of Pharmacology and Experimental Therapeutics 300: 831.

D'Addario, C., Di Benedetto, M., Izenwasser, S., Candeletti, S., and Romualdi, P. 2007. Role of serotonin in the regulation of the dynorphinergic system by a $\kappa$-opioid agonist and cocaine treatment in rat CNS. Neuroscience 144: 157-164.

Dackis, C., Kampman, K.M., Lynch, K.G., Pettinati, H.M., and O'Brien, C. 2005. A double-blind, placebo-controlled trial of modafinil for cocaine dependence. Neuropsychopharmacology 30: 205-211.

Dackis, C., and O'Brien, C. 2006. Glutamatergic agents for cocaine dependence. Annals New York Academy of Sciences 1003: 328-345.

Dal Toso, R., Sommer, B., Ewert, M., Herb, A., Pritchett, D.B., Bach, A., Shivers, B.D., and Seeburg, P.H. 1989. The dopamine D2 receptor: two molecular forms generated by alternative splicing. The EMBO Journal 8: 4025-4034.

Daniels, G.M., and Amara, S.G. 1999. Regulated trafficking of the human dopamine transporter: clatherin-mediated internalization and lysosomal degradation in response to phorbol esters. Journal of Biological Chemistry 274: 35794-35801.

Daws, L.C., Callaghan, P.D., Moron, J.A., Kahlig, K.M., Shippenberg, T.S., Javitch, J.A., and Galli, A. 2002. Cocaine increases dopamine uptake and cell surface expression of dopamine transporters. Biochemical and Biophysical Research Communications 290: 1545-1550.

Daws, L.C., Montanez, S., Owens, W.A., Gould, G.G., Frazer, A., Toney, G.M., and Gerhardt, G.A. 2005. Transport mechanisms governing serotonin clearance in vivo revealed by high-speed chronoamperometry. Journal of Neuroscience Methods 143: 49-62.

Deering, D., Sellman, D., Adamson, S., Campbell, S., Sheridan, J., Pooley, S., Robertson, R., and Henderson, C. 2008. Intravenous opioid dependence in new zealand. (ed. MOH). MOH, Auckland.

Di Chiara, G., and Imperato, A. 1988a. Drugs abused by humans preferentially increase synaptic dopamine concentrations in the mesolimbic system of freely moving rats. Proceedings of the National Academy of Sciences of the United States of America 85: 5274-5278.

Di Chiara, G., and Imperato, A. 1988b. Opposite effects of $m u$ and kappa opiate agoints on dopamine release in the nucleus accumbens and in the dorsal caudate of freely moving rats. The Journal of Pharmacology and Experimental Therapeutics 244: 1067-1080.

Diaz, J., Pilon, C., Le Foll, B., Gros, C., Triller, A., Schwartz, J.-C., and Sokoloff, P. 2000. Dopamine $\mathrm{D}_{3}$ receptors expressed by all mesencephalic dopamine neurons. The Journal of Neuroscience 20: 8677-8684.

Doolen, S., and Zahniser, N.R. 2001. Protein tyrosine kinase inhibitors alter human dopamine transporter activity in Xenopus oocytes. The Journal of Pharmacology and Experimental Therapeutics 296: 931-938.

Earles, C., and Schenk, J.O. 1998. Rotating disk electrode voltammetric measurements of dopamine transporter activity: an analytical evaluation. Analytical Biochemistry 264: 191-198.

Earles, C., and Schenk, J.O. 1999a. Multisubstrate mechanism for the inward transport of dopamine by the human dopamine transporter expressed in HEK cells and its inhibition by cocaine. Synapse 33: 230-238.

Earles, C., and Schenk, J.O. 1999b. Multisubtrate mechanism for the inward transport of dopamine by the human dopamine transporter expressed in HEK cells and its inhibition by cocaine. Synapse 33: 230-238. 
Earles, C., Wayment, H., Green, M., and Schenk, J.O. 1998. Resolution of biogenic amine transporter kinetics by rotating disk electrode voltammetry: methodology and mechanistic interpretations. In Neurotransmitter Transporters, pp. 660-675.

Ebner, S.R., Roitman, M.F., Potter, D., Rachlin, A.B., and Chartoff, E.H. 2010. Depressive-like effects of the kappa opioid receptor agonist salvinorin A are associated with decreased phasic dopamine release in the nucleus accumbens. Psychopharmacology 210: 241-252.

El Daly, E., Chefer, V.I., Sandill, S., and Shippenberg, T.S. 2000. Modulation of the neurotoxic effects of methamphetamine by the selective $\kappa$-opioid receptor agonist U69593. Journal of Neurochemistry 74: 1553-1562.

Elliot, J.M., and Beveridge, T.J. 2005. Psychostimulants and monoamine transporters: upsetting the balance. Current Opinion in Pharmacology 5: 94-100.

Emmerson, P.J., Liu, M.-R., Woods, J.H., and Medzihradsky, F. 1994. Binding affinity and selectivity of opioids at $m u$, delta and kappa receptors in monkey brain membranes. The Journal of Pharmacology and Experimental Therapeutics 271: 1630-1637.

Fantegrossi, W.E., Kugle, K.M., Valdes, L.J., Koreeda, M., and Woods, J.H. 2005. Kappa-opioid receptor-mediated effects of the plant-derived hallucinogen, salvinorin A, on inverted screen performance in the mouse. Behavioural Pharmacology 16: 627-633.

Feinn, R., Nellissery, M., and Kranzler, H.R. 2005. Meta-analysis of the association of a functional serotonin transporter promoter polymorphism with alcohol dependence. American Journal of Medical Genetics (Neuropsychiatric Genetics) 133B: $79-84$.

Fichna, J., Schicho, R., Andrews, C.N., Bashashati, M., Klompus, M., McKay, D.M., Sharkey, K.A., Zjawiony, J.K., Janecka, A., and Storr, M.A. 2009. Salvinorin A inhibits colonic transit and neurogenic ion transport in mice by activating $\mathrm{K}$ opioid and cannabinoid receptors. Neurogastroenterol. Motil. 21: 1326-13337.

Filip, M., and Cunningham, K.A. 2003. Hyperlocomotive and discriminative stimulus effects of cocaine are under the control of serotonin $2 \mathrm{C}\left(5-\mathrm{HT}_{2 \mathrm{C}}\right)$ receptors in rat prefrontal cortex. The Journal of Pharmacology and Experimental Therapeutics 306: $734-743$.

Flatmark, T. 2000. Catecholamine biosynthesis and physiological regulation in neuroendocrine cells. Acta Physiologica Scandinavica 168: 1-17.

Fog, J.U., Khoshbouei, H., Holy, M., Owens, W.A., Vaegter, C.B., Sen, N., Nikandrova, Y., Bowton, E., McMahon, D.G., Colbran, R.J., et al. 2006. Calmodulin kinase II interacts with the dopamine transproter $\mathrm{C}$ terminus to regulate amphetamine-induced reverse transport. Neuron 51: 417-429.

Forrest, L.R., Tavoulari, S., Zhang, Y.-W., Rudnick, G., and Honig, B. 2007. Identification of a chloride ion binding site in $\mathrm{Na}^{+} / \mathrm{Cl}^{-}$dependent transporters. Proceedings of the National Academy of Sciences of the United States of America 104: 12761-12766.

Foster, J.D., Adkins, S.D., Lever, J.R., and Vaughan, R.A. 2008. Phorbal ester induced trafficking-independent regulation and enhanced phosphorylation of the dopamine transporter associated with membrane rafts and cholesterol. Journal of Neurochemistry 105: 1683-1699.

Frankel, P.S., Alburges, M.E., Bush, L., Hanson, G.R., and Kish, S.J. 2008. Striatal and ventral pallidum dynorphin concentrations are markedly increased in human chronic cocaine users. Neuropharmacology 55: 41-46.

Fuentealba, J.A., Gysling, K., and Andres, M.A. 2007. Increased locomotor response to amphetamine induced by the repeated administration of the selective kappaopioid receptor agonist U-69593. Synapse 61: 771-777. 
Fuentealba, J.A., Gysling, K., and Andres, M.A. 2010. Repeated treatment with the $\kappa-$ opioid agonist U-69593 increases $\mathrm{K}^{+}$-stimulated dopamine release in the rat medial prefrontal cortex. Synapse 64: 898-904.

Fuentealba, J.A., Gysling, K., Magendzo, K., and Andres, M.E. 2006. Repeated administration of the selective kappa-opioid receptor agonist U-69593 increases stimulated dopamine extracellular levels in the rat nucleus accumbens. Journal of Neuroscience Research 84: 450-459.

Fukuda, K., Kato, S., Morikawa, H., Shoda, T., and Mori, K. 1996. Functional coupling of the $\delta$-, $\mu$-, and $\kappa$-opioid receptors to mitogen-activated protein kinase and arachidonate release in chinese hamster ovary cells. Journal of Neurochemistry 67: 1309-1316.

Fuller, R.K. 2004. Does disulfiram have a role in alcoholism treatment today? Addiction 99: 21-24.

Furman, C.A., Chen, R., Guptaroy, B., Zhang, M., Holz, R.W., and Gnegy, M. 2009a. Dopamine and amphetamine rapidly increase dopamine transporter trafficking to the surface: live-cell imaging using total internal reflection fluorescence microscopy. Journal of Neuroscience 29: 3328-3336.

Furman, C.A., Lo, C.B., Stokes, S., Esteban, J.A., and Gnegy, M. 2009b. Rab 11 regulates constitutive dopamine transporter trafficking and function in $\mathrm{N} 2 \mathrm{~A}$ neuroblastoma cells. Neuroscience Letters 463: 78-81.

Gehrke, B.J., Chefer, V.I., and Shippenberg, T.S. 2008. Effects of acute and repeated administration of salvinorin A on dopamine function in the rat dorsal striatum. Psychopharmacology (Berl) 197: 509-517.

Gerra, G., Garofano, L., Santoro, G., Bosari, S., Pellegrini, C., Zaimovic, A., Moi, G., Bussandri, M., Moi, A., Brambilla, F., et al. 2004. Association between lowactivity serotonin transporter genotype and heroin dependence: behavioral and personality correlates. American Journal of Medical Genetics (Neuropsychiatric Genetics) 126B: 37-42.

Giros, B., Mestitkawy, S.E., Godinot, N., Zheng, K., Han, H., T., Y.-F., and Caron, M.G. 1992. Cloning, pharmacological characterization, and chromosome assignment of the human dopamine transporter. Molecular Pharmacology 42: 383-390.

Glick, S.D., Maisonneuve, I.M., Raucci, J., and Archer, S. 1995. Kappa opioid inhibition of morphine and cocaine self-administration in rats. Brain Research 681: $147-152$.

Goldman, N., Glei, D.A., Lin, Y.-H., and Weinstein, M. 2010. The serotonin transporter polymorphism (5-HTTLPR): allelic variation and links with depressive symptoms. Depression and Anxiety 27: 260-269.

Goldstein, A., and Ghazarossian, V.E. 1980. Immunoreactive dynorphin in pituitary and brain. Proceedings of the National Academy of Sciences of the United States of America 77: 6207-6210.

Gonzalez, D., Riba, J., Bouso, J.C., Gomez-Jarabo, G., and Barbanoj, M.J. 2006. Pattern of use and subjective effects of Salvia divinorum among recreational users. Drug and Alcohol Dependence 85: 157-162.

Granas, C., Ferrer, J., Loland, C.J., Javitch, J.A., and Gether, U. 2003. N-terminal truncation of the dopamine transporter abolishes phorbol ester- and substance $\mathrm{P}$ receptor-stimulated phosphorylation without impairing transporter internalization. Journal of Biological Chemistry 278: 4990-5000.

Gray, A.M., Rawls, S.M., Shippenberg, T.S., and McGinty, J.F. 1999. The kappa-opioid agonist, U-69593, decreases acute amphetamine-evoked behaviors and calciumdependent dialysate levels of dopamine and glutamate in the ventral striatum. Journal of Neurochemistry 73: 1066-1074. 
Grilli, M., Neri, E., Zappettini, S., Massa, F., Bisio, A., Romussi, G., Marchi, M., and Pittaluga, A. 2009. Salvinorin A exerts opposite presynaptic controls on neurotransmitter exocytosis from mouse brain nerve terminals. Neuropharmacology 57: 523-530.

Groer, C.E., Tidgewell, K., Moyer, R.A., Harding, W.W., Rothman, R.B., Prisinzano, T.E., and Bohn, L.M. 2007. An opioid agonist that does not induce $\mu$-opioid receptor-arrestin interactions or receptor internalization. Molecular Pharmacology 71: 549-557.

Gu, H., Wall, S.C., and Rudnick, G. 1994. Stable expression of biogenic amine transporters reveal differences in inhibitor sensitivity, kinetics, and ion dependence. The Journal of Biological Chemistry 269: 7124-7130.

Gudehithlu, K.P., and Bhargava, H.N. 1996. Modification of the characteristics of dopamine transporter in brain regions and spinal cord of morphine tolerant and abstinent rats. Neuropharmacology 35: 169-174.

Guindalini, C., Howard, M., Haddley, K., Laranjeira, R., Collier, D., Ammar, N., Craig, I., O'Gara, C., Bubb, V.J., Greenwood, T., et al. 2006. A dopamine transporter gene functional variant associated with cocaine abuse in a Brazilian sample. Proceedings of the National Academy of Sciences of the United States of America 103: 4552-4557.

Gulley, J.M., Doolen, S., and Zahniser, N.R. 2002. Brief, repeated exposure to substrates down-regulates dopamine transporter function in Xenopus oocytes in vitro and rat dorsal striatum in vivo. Journal of Neurochemistry 83: 400-411.

Guo, S., Chen, d.F., Zhou, D.-F., Sun, H.Q., Wu, G.Y., Haile, C.N., Kosten, T.A., Kosten, T.R., and Zhang, X.Y. 2007. Association of functional catechol-Omethyl transferase (COMT) val108met polymorphism with smoking severity and age of smoking initiation in Chinese male smokers. Psychopharmacology 190: $449-456$.

Hagan, C.E., Neumaier, J.F., and Schenk, J.O. 2010. Rotating disk electrode voltammetric measurements of serotonin transporter kinetics in synaptosomes. Journal of Neuroscience Methods 193: 29-38.

Hagiwara, H., Suka, Y., Nojima, T., Hoshi, T., and Suzuki, T. 2009. Second-generation synthesis of salvinorin A. Tetrahedron 65: 4820-4825.

Hahn, B., Stolerman, I.P., and Shoaib, M. 2000. Kappa-opioid receptor modulation of nicotine-induced behaviour. Neuropharmacology 39: 2848-2855.

Hahn, J.W., Jagwani, S., Kim, E., Rendell, V.R., He, J., Ezerskiy, L.A., Wesselschmidt, R., Coscia, C.J., and Belcheva, M.M. 2010. Mu and kappa opioids modulate mouse embryonic stem cell-derived neural progenitor differentiation via MAP kinases. Journal of Neurochemistry 112: 1431-1441.

Hales, J., and Manser, J. 2007. New Zealand police NZ-ADAM annual report 2007. Health Outcomes International Pty Ltd, Kent Town, Southern Australia.

Hanes, K.R. 2001. Antidepressant effects of the herb salvia divinorum: a case report. Journal of Clinical Psychopharmacology 21: 634-635.

Harding, W.W., Schmidt, M., Tidgewell, K., Kannan, P., Holden, K.G., Dersch, C.M., Rothman, R.B., and Prisinzano, T.E. 2006. Synthetic studies of neoclerodane diterpenes from Salvia divinorum: selective modification of the furan ring. Bioorganic \& Medicinal Chemistry Letters 16: 3170-3174.

Harding, W.W., Tidgewell, K., Byrd, N., Cobb, H., Dersch, C.M., Butelman, E.R., Rothman, R.B., and Prisinzano, T.E. 2005. Neoclerodane diterpenes as a novel scaffold for mu opioid receptor ligands. Journal of Medicinal Chemistry 48: 4765-4771.

Hastrup, H., Karlin, A., and Javitch, J.A. 2001. Symmetrical dimer of the human dopamine transporter revealed by cross-linking Cys-306 at the extracellular end 
of the sixth transmembrane segment. Proceedings of the National Academy of Sciences of the United States of America 98: 10055-10060.

Heidbreder, C.A., Goldberg, S.R., and Shippenberg, T.S. 1993. The kappa-opioid receptor agonist U-69593 attenuates cocaine-induced behavioral sensitization in the rat. Brain Research 616: 335-338.

Heidbreder, C.A., Schenk, S., Partridge, B., and Shippenberg, T.S. 1998. Increased responsiveness of mesolimbic and mesostriatal dopamine neurons to cocaine following repeated administration of a selective kappa-opioid receptor agonist. Synapse 30: 255-262.

Heidbreder, C.A., and Shippenberg, T.S. 1994. U-69593 prevents cocaine sensitization by normalizing basal accumbens dopamine. Neuroreport 5: 1797-1800.

Heyliger, S.O., Jackson, C., Rice, K.C., and Rothman, R.B. 1999. Opioid peptide receptor studies. 10. nor-BNI differentially inhibits kappa receptor agonistinduced G-protein activation in the guinea pig caudate: further evidence of kappa receptor heterogeneity. Synapse 34: 256-265.

Hoffman, B.J., Hansson, S.R., Mezey, E., and Palkovits, M. 1998. Localization and dynamic regulation of biogenic amine transporters in the mammalian central nervous system. Frontiers in Neuroendocrinology 19: 187-231.

Holden, K.G., Tidgewell, K., Marquam, A., Rothman, R.B., Navarro, H., and Prisinzano, T.E. 2007. Synthetic studies of neoclerodane diterpenes from Salvia divinorum: exploration of the 1-position. Bioorganic \& Medicinal Chemistry Letters 17: 6111-6115.

Hooker, J.M., Patel, V., Kothari, S., and Schiffer, W.K. 2009. Metabolic changes in the rodent brain after acute administration of salvinorin A. Molecular Imaging and Biology 11: 137-143.

Hooker, J.M., Xu, Y., Schiffer, W., Shea, C., Carter, P., and Fowler, J.S. 2008. Pharmacokinetics of the potent hallucinogen, salvinorin A in primates parallels the rapid onset and short duration of effects in humans. NeuroImage 41: 10441050 .

Hoover, B.R., Everett, C.V., Sorkin, A., and Zahniser, N.R. 2007. Rapid regulation of dopamine transporters by tyrosine kinases in rat neuronal preparations. Journal of Neurochemistry 101: 1258-1271.

Horan, P.J., de Costa, B.R., Rice, K., Haaseth, R.C., Hruby, V.J., and Porreca, F. 1993. Differential antagonism of bremazocine- and the U69,593-induced antinociception by quadazocine: further functional evidence of opioid $\kappa$ receptor multiplicity in the mouse. Journal of Pharmacology and Experimental Therapeutics 266: 926-933.

Horan, P.J., Taylor, J., Yamamura, H.I., and Porreca, F. 1992. Extremely long-lasting antagonist actions of nor-binaltorphimine (nor-BNI) in the mouse tail-flick test. The Journal of Pharmacology and Experimental Therapeutics 260: 1237-1243.

Horowitz, R., Kotler, M., Shufman, E., Aharoni, S., Kremer, I., Cohen, H., and Ebstein, R.P. 2000. Confirmation of an excess of the high enzyme activity COMT val allele in heroin addicts in a family-based haplotype relative risk study. American Journal of Medical Genetics (Neuropsychiatric Genetics) 96: 599-603.

Horvath, M.C., Kovacs, G.G., Kovarl, V., Majtenyl, K., Hurd, Y.L., and Keller, E. 2007. Heroin abuse is characterized by discrete mesolimbic dopamine and opioid abnormalities and exaggerated nuclear receptor-related 1 transcriptional decline with age. The Journal of Neuroscience 27: 13371-13375.

Howell, L.L., and Kimmel, H.L. 2008. Monoamine transporters and psychostimulant addiction. Biochemical Pharmacology 75: 196-217. 
Hsu, Y.-P., Loh, E.W., Chen, W.J., Chen, C.-C., Yu, J.-M., and Cheng, A.T.A. 1996. Association of monoamine oxidase A alleles with alcoholism among male chinese in taiwan. The American Journal of Psychiatry 153: 1209-1211.

Huang, S.H., Lifshitz, L.M., Jones, C., Bellve, K.D., Standley, C., Fonseca, S., Corvera, S., Fogarty, K.E., and Czech, M.P. 2007. Insulin stimulates membrane fusion and GLUT4 accumulation in clathrin coats on adipocyte plasma membranes. Molecular and Cellular Biology 27: 3456-3469.

Huang, W., Payne, T.J., Ma, J.Z., Beuten, J., Dupont, R.T., Inohara, N., and Li, M.D. 2009a. Significant association of ANKK1 and detection of a functional polymorphism with nicotine dependence in an african-american sample. Neuropsychopharmacology 34: 319-330.

Huang, W., Payne, T.J., Ma, J.Z., and Li, M.D. 2008. A functional polymorphism, rs6280, in DRD3 is significantly associated with nicotine dependence in european-american smokers. American Journal of Medical Genetics (Neuropsychiatric Genetics) 147B: 1109-1115.

Huang, X., Gu, H., and Zhan, C.-G. 2009b. Mechanism for cocaine blocking the transport of dopamine: insights from molecular modeling and dynamics simulations. The journal of Physical Chemistry B 113: 15057-15066.

Huff, R.A., Vaughan, R.A., Kuhar, M.J., and Uhl, G.R. 1997. Phorbol esters increase dopamine transporter phosphorylation and decrease transport $\mathrm{V}_{\max }$. Journal of Neurochemistry 68: 225-232.

Hulse, G.K., Morris, N., Arnold-Reed, D., and Tait, R.J. 2009. Improving clinical outcomes in treating heroin dependence randomized, controlled trial of oral or implant naltrexone. Archives of General Psychiatry 66: 1108-1115.

Hurd, Y.L., and Herkenham, M. 1993. Molecular alterations in the neostriatum of human cocaine addicts. Synapse 13: 357-369.

Inan, S., Lee, D.Y.-W., Liu-Chen, L.Y., and Cowan, A. 2009. Comparison of the diuretic effects of chemically diverse kappa opioid agonists in rats; nalfurafine, $\mathrm{U} 50,488 \mathrm{H}$, and salvinorin A. Naunyn-Schmiedeberg's Archives of Pharmacology 379: 263-270.

Izenwasser, S., Acri, J.B., Kunko, P.M., and Shippenberg, T. 1998. Repeated treatment with the selective kappa opioid agonist U-69593 produces a marked depletion of dopamine D-2 receptors. Synapse 30: 275-283.

Jackisch, R., Hotz, H., Allgaier, C., and Hertting, G. 1994. Presynaptic opioid receptors on dopaminergic nerves in the rabbit caudate nucleus: coupling to pertussis toxin-sensitive G-proteins and interaction with D2 autoreceptors? NaunynSchmiedeberg's Archives of Pharmacology 349: 250-258.

Jermain, J.D., and Evans, H.K. 2009. Analyzing salvia divinorum and its active ingredient salvinorin A utilizing thin layer chromatography and gas chromatography/mass spectrometry. Journal of Forensic Sciences 54: 612-616.

Jin, Y., Chen, D., Hu, Y., Guo, S., Sun, H., Lu, A., Zhang, X., and Li, L. 2006. Association between monoamine oxidase gene polymorphisms and smoking behavior in Chinese males. International Journal of Neuropsychopharmacology 9: $557-564$

John, C.E., and Jones, S.R. 2007a. Voltammetric characterization of the effect of monoamine uptake inhibitors and releasers on dopamine and serotonin uptake in mouse caudate-putamen and substantia nigra slices. Neuropharmacology 52: 1596-1605.

John, C.E., and Jones, S.R. 2007b. Voltammetric characterization of the effect of monoamine uptake inhibitors and releasers on dopamine and serotonin uptake in mouse caudate-putamen and substantia nigra slices. Neuropharmacology 52: 1596-1605. 
John, T.F., French, L.G., and Erlichman, J.S. 2006. The antinociceptive effect of salivonorin A in mice. European Journal of Pharmacology 545: 129-133.

Johnson, L.A., Guptaroy, B., Lund, D., Shamban, S., and Gnegy, M. 2005. Regulation of amphetamine-stimulated dopamine efflux by protein kinase C $\beta$. Journal of Biological Chemistry 280: 10914-10919.

Jones, B.J., and Blackburn, T.P. 2002. The medical benefit of 5'HT research. Pharmacology, Biochemistry and Behavior 71: 555-568.

Jones, R.M., and Portoghese, P.S. 2000. 5'-guanidinonaltrindole, a highly selective and potent $\kappa$-opioid receptor antagonist. European Journal of Pharmacology 396: 49-52.

Jonsson, E.G., Nothen, M.M., Gruhage, F., Farde, L., Nakashima, Y., Propping, P., and Sedvall, G.C. 1999. Polymorphisms in the dopamine D2 receptor gene and their relationships to striatal dopamine receptor density of healthy volunteers. Molecular Psychiatry 4: 290-296.

Jordan, B.A., Cvejic, S., and Devi, L.A. 2000. Kappa opioid receptor endocytosis by dynorphin peptides. DNA and Cell Biology 19: 19-27.

Jordan, B.A., and Devi, L.A. 1999. G-protein-coupled receptor heterodimerization modulates receptor function. Nature 399: 697-700.

Jorenby, D.E., Hays, J.T., Rigotti, N.A., Azoulay, S., Watsky, E.J., WIlliams, K.E., Billing, C.B., Gong, J., and Reeves, K.R. 2006. Efficacy of varenicline, an $\alpha 4 \beta 2$ nicotinic acetylcholine receptor partial agonist, vs placebo or sustained-release bupropion for smoking cessation. The Journal of the American Medicial Association 296: 56-63.

Joseph, J.D., Wang, Y.M., Miles, P.R., Budygin, E.A., Picetti, R., Gainetdinov, R.R., Caron, M.G., and Wightman, R.M. 2002. Dopamine autoreceptor regulation of release and uptake in mouse brain slices in the absence of D3 receptors. Neuroscience 112: 39-49.

Kahlig, K.M., Binda, F., Khoshbouei, H., Blakely, R.D., McMahon, D.G., Javitch, J.A., and Galli, A. 2005. Amphetamine induces dopamine efflux through a dopamine transporter channel. Proceedings of the National Academy of Sciences of the United States of America 102: 3495-3500.

Kalyuzhyny, A.E., and Wessendorf, M.W. 1999. Serotonergic and GABAergic neurons in the medial rostral ventral medulla express $\kappa$-opioid receptor immunoreactivity. Neuroscience 90: 229-234.

Kam, A.Y.F., Chan, A.S.L., and Wong, Y.H. 2004a. א-opioid receptor signals through src and focal adhesion kinase to stimulate c-jun $\mathrm{N}$-terminal kinases in transfected COS-7 cells and human monocytic THP-1 cells. The Journal of Pharmacology and Experimental Therapeutics 310: 301-310.

Kam, A.Y.F., Chan, A.S.L., and Wong, Y.H. 2004b. Phosphatidylinositol-2 kinase is distinctively required for $\mu$ - but not $\kappa$-opioid receptor-induced activation of $\mathrm{c}$ Jun N-terminal kinase. Journal of Neurochemistry 89: 391-402.

Kane, B.E., McCurdy, C.R., and Ferguson, D.M. 2008. Toward a structure-based model of salvinorin a recognition of the kappa-opioid receptor. Journal of Medicinal Chemistry 51: 1824-1830.

Kane, B.E., Nieto, M.J., McCurdy, C.R., and Ferguson, D.M. 2006. A unique binding epitope for salvinorin A, a non-nitrogenous kappa opioid receptor agonist. Febs Journal 273: 1966-1974.

Kennedy, J.H., and Wiseman, J.M. 2010. Direct analysis of Salvia divinorum leaves for salvinorin A by thin layer chromatography and desorption electrospray ionization multi-stage tandem mass spectrometry. Rapid Communications in Mass Spectrometry 24: 1305-1311. 
Kenworthy, A.K., Nichols, B.J., Remmert, C.L., Hendrix, G.M., Kumar, M., Zimmerberg, J., and Lippincott-Schwartz, J. 2004. Dynamics of putative raftassociated proteins at the cell surface. Journal of Cell Biology 165: 735-746.

Khokhar, J.Y., Ferguson, C.S., Zhu, A.Z.X., and Tyndale, R.F. 2010. Pharmacogenetics of drug dependence: role of gene variations in susceptibility and treatment. Annual Review of Pharmacology \& Toxicology 50: 39-61.

Khoshbouei, H., Sen, N., Guptaroy, B., Johnson, L., Lund, D., Gnegy, M., Galli, A., and Javitch, J.A. 2004. N-terminal phosphorylation of the dopamine transporter is required for amphetamine-induced efflux. PLoS Biology 2: 0387-0393.

Killinger, B.A., Peet, M.M., and Baker, L.S. 2010. Salvinorin A fails to substitute for the discriminative stimulus effects of LSD or ketamine in Sprague-Dawley rats. Pharmacology Biochemistry and Behavior 96: 260-265.

Kilty, J.E., Lorang, D., and Amara, S.G. 1991. Cloning and expression of a cocainesensitive rat dopamine transporter. Science 254: 578-579.

Kim, E., Clark, A.L., Kiss, A., Hahn, J.W., Wesselschmidt, R., Coscia, C.J., and Belcheva, M.M. 2006. $\mu$ - and א-opioids induce the differentiation of embryonic stem cells to neural progenitors. The Journal of Biological Chemistry 281: 33749-33760.

Kirsch, I., Deacon, B.J., Huedo-Medina, T.B., Scoboria, A., Moore, T.J., and Johnson, B.T. 2008. Initial severity and antidepressant benefits: a meta-analysis of data submitted to the Food and Drug Administration. PLoS Med 5: e45.

Kish, S.J., Kalasinsky, K.S., Derkach, P., Schmunk, G.A., Guttman, M., Ang, L., Adams, V., Furukawa, Y., and Haycock, J. 2001. Striatal dopaminergic and serotonergic markers in human heroin users. Neuropsychopharmacology 24: 561-567.

Kivell, B., Day, D., Bosch, P., Schenk, S., and Miller, J. 2010. MDMA causes a redistribution of serotonin transporter from the cell surface to the intracellular compartment by a mechanism independent of phospho-p38-mitogen activated protein kinase activation. Neuroscience 168: 82-95.

Kivell, B., Kahlig, K., Galli, A., Javitch, J.A., and Shippenberg, T. 2004. Regulation of dopamine transporter function and cell surface expression by kappa opioid receptors. Society for Neuroscience Abstract Viewer and Itinerary Planner 280.12.

Klitenick, M.A., DeWitte, P., and Kalivas, P.W. 1992. Regulation of somatodendritic dopamine release in the ventral tegmental area by opioids and GABA: an in vivo microdialysis study. Journal of Neuroscience 12: 2623-2632.

Knoll, A.T., Meloni, E.G., Thomas, J.B., Carroll, F.I., and Carlezon, W.A. 2007. Anxiolyticlike effects of kappa-opioid receptor antagonists in models of unlearned and learned fear in rats. Journal of Pharmacology and Experimental Therapeutics 323: 838-845.

Kokoshka, J.M., Metzger, R.R., Wilkins, D.G., Gibb, J.W., Hanson, G.R., and Fleckenstein, A.E. 1998. Methamphetamine treatment rapidly inhibits serotonin, but not glutamate, transporters in rat brain. Brain Research 799: 78-83.

Koob, G. 2008. A role for brain stress systems in addiction. Neuron 59: 11-34.

Koob, G., and Kreek, M.J. 2007. Stress, dysregulation of drug reward pathways, and the transition to drug dependence. American Journal of Psychiatry 164: 1149-1159.

Koob, G., and Le Moal, M. 1997. Drug abuse: hedonic homeostatic dysregulation. Science 278: 52-58.

Koob, G.F., and Le Moal, M. 2001. Drug addiction, dysregulation of reward, and allostasis. Neuropsychopharmacology 24: 97-129.

Korlach, J., Schwille, P., Webb, W.W., and Feigenson, G.W. 1999. Characterization of lipid bilayer phases by confocal microscopy and fluorescence correlation 
spectroscopy. Proceedings of the National Academy of Sciences of the United States of America 96: 8461-8466.

Kunihara, M., Ohyama, M., and Nakano, M. 1993. Effects of spiradoline mesylate, a selective $\kappa$-opioid-receptor agonist, on the central dopamine system with relation to mouse locomotor activity and analgesia. Japanese Journal of Pharmacology 62: 223-230.

La Regina, A., Petrillo, P., Sbacchi, M., and Tavani, A. 1988. Interaction of U-69,593 with $\mathrm{m}-$, d- and k-opioid binding sites and its analgesic and intestinal effects in rats. Life Sciences 42: 293-301.

Laine, T.P.J., Ahonen, A., Torniainen, P., Heikkila, J., Pyhtinen, J., Rasanen, P., Niemela, O., and Hillbom, M. 1999. Dopamine transporters increase in human brain after alcohol withdrawal. Molecular Pyschiatry 4: 189-195.

Land, B.B., Bruchas, M.R., Schattauer, S., Giardino, W.J., Aita, M., Messinger, D., Hnasko, T.S., Palmiter, R.D., and Chavkin, C. 2009. Activation of the kappa opioid receptor in the dorsal raphe nucleus mediates the aversive effects of stress and reinstates drug seeking. Proceedings of the National Academy of Sciences of the United States of America 106: 19168-19173.

Lange, J.E., Reed, M.B., Ketchie Croff, J.M., and Clapp, J.D. 2008. College student use of Salvia divinorum. Drug and Alcohol Dependence 94: 263-266.

Laruelle, M., Gelernter, J., and Innis, R.B. 1998. $\mathrm{D}_{2}$ receptors binding potential is not affected by Taq1 polymorphism at the $\mathrm{D}_{2}$ receptor gene. Molecular Psychiatry 3: 261-265.

Laucht, M., Treutlein, J., Blomeyer, D., Buchmann, A.F., Schmid, B., Becker, K., Zimmermann, U.S., Schmidt, M.H., Esser, G., Rietschel, M., et al. 2009. Interaction between the 5-HTTLPR serotonin transporter polymorphism and environmental adversity for mood and anxiety psychopathology: evidence from a high-risk community sample of young adults. International Journal of Neuropsychopharmacology 12: 737-747.

Lawford, B.R., Young, R.M., Noble, E.P., Sargent, J., Rowell, J., Shadforth, S., ZHang, X., and Ritchie, T.L. 2000. The $\mathrm{D}_{2}$ dopamine receptor $\mathrm{A}_{1}$ allele and opioid dependence: association with heroin use and response to methadone treatment. American Journal of Medical Genetics (Neuropsychiatric Genetics) 96: 592598.

Lawrence, J.C., Saslowsky, D.E., Edwardson, J.M., and Henderson, R.M. 2003. Realtime analysis of the effects of cholesterol on lipid raft behavior using atomic force microscopy. Biophysical Journal 84: 1827-1832.

Lee, D.Y.W., He, M.S., Liu-Chen, L.Y., Wang, Y.L., Li, J.G., Xu, W., Ma, Z.Z., Carlezon, W.A., and Cohen, B. 2006. Synthesis and in vitro pharmacological studies of new C(4) modified salvinorin A analogues. Bioorganic \& Medicinal Chemistry Letters 16: 5498-5502.

Lee, D.Y.W., Yang, L., Xu, W., Deng, G., Guo, L., and Liu-Chen, L.-Y. 2010. Synthesis and biological evaluation of C-2 halogenated analogs of salvinorin A. Bioorganic \& Medicinal Chemistry Letters 20: 5749-5752.

Lee, F.J.S., Pei, L., Moszczynska, A., Vukusic, B., Fletcher, P.J., and Liu, F. 2007. Dopamine transporter cell surface localization facilitated by a direct interaction with the dopamine D2 receptor. The EMBO Journal 26: 2127-2136.

Leppa, S., and Bohmann, D. 1999. Diverse functions of JNK signaling and c-Jun in stress response and apoptosis. Oncogene 18: 6158-6162.

Lerman, C., Caporaso, N.E., Audrain, J., Main, D., Bowman, E.D., Lockshin, B., Boyd, N.R., and Shields, P.G. 1999. Evidence suggesting the role of specific genetic factors in cigarette smoking. Health Psychology 18: 14-20. 
Letchworth, S.R., Nader, M.A., Smith, H.R., Friedman, D.P., and Porrino, L.J. 2001. Progression of changes in dopamine transporter binding site density as a result of cocaine self-administration in rhesus monkeys. The Journal of Neuroscience 21: 2799-2807.

Levesque, D., Diaz, J., Pilon, C., Martres, M.-P., Giros, B., Souil, E., Schott, D., Morgat, J.-L., Schwartz, J.-C., and Sokoloff, P. 1992. Identification, characterization, and localization of the dopamine D3 receptor in rat brain using 7- $\left[{ }^{3} \mathrm{H}\right]$ hydroxy- $N, N$-di-n-propyl-2-aminotetralin. Proceedings of the National Academy of Sciences of the United States of America 89: 8155-8159.

Li, C., Zhong, H., Wang, Y., Wang, H., Yang, Z., Zheng, Y.L., K., and Liu, Y. 2006. Voltage and ionic regulation of human serotonin transporter in xenopus oocytes. Clinical and Experimental Pharmacology and Physiology 33: 1088-1092.

Li, C.H., Bai, L., Li, D.D., Xia, S., and Xu, T. 2004a. Dynamic tracking and mobility analysis of single GLUT4 storage vesicle in live 3T3-L1 cells. Cell Research 14: 480-486.

Li, J., Li, J.-G., Chen, C., Zhang, F., and Liu-Chen, L.-Y. 2002. Molecular basis of differences in (-)(trans)-3,4-dichloro- $N$-methyl- $N$-[2-(1-pyrrolidiny)cyclohexyl]benzeneacetamide induced desensitization and phosphorylation between human and rat $\kappa$-opioid receptors expressed in chinese hamster ovary cells. Molecular Pharmacology 61: 73-84.

Li, J.G., Luo, L.Y., Krupnick, J.G., Benovic, J.L., and Liu-Chen, L.Y. 1999. U50,488Hinduced internalization of the human kappa opioid receptor involves a betaarrestin- and dynamin-dependent mechanism - kappa receptor internalization is not required for mitogen-activated protein kinase activation. Journal of Biological Chemistry 274: 12087-12094.

Li, L.-B., Chen, N., Ramamoorthy, S., Chi, L., Cui, X.-N., Wang, L.C., and Reith, M.E.A. 2004b. The role of $N$-glycosylation in function and surface trafficking of the human dopamine transporter. The Journal of Biological Chemistry 279: 21012-21020.

Li, S., Kim, K.Y., Kim, J.H., Kim, J.H., Park, M.S., Bahk, J.Y., and Kim, M.O. 2004c. Chronic nicotine and smoking treatment increases dopamine transporter mRNA expression in the rat midbrain. Neuroscience Letters 363: 29-32.

Lin, A.M.-Y., and Chai, C.-Y. 1995. Dynamic analysis of ethanol effects on NMDAevoked dopamine overflow in rat striatum. Brain Research 696: 15-20.

Lindgren, N., Uslello, A., Goiny, M., Haycock, J., Erbs, E., Greengard, P., Hokfelt, T., Borelli, E., and Fisone, G. 2003. Distinct roles of dopamine D2L and D2S receptor isoforms in the regulation of protein phosphorylation at presynaptic and postsynaptic sites. Proceedings of the National Academy of Sciences of the United States of America 100: 4305-4309.

Ling, D., Niu, T., Feng, Y., Xing, H., and Xu, X. 2004. Association between polymorphism of the dopamine transporter gene and early smoking onset: an interaction risk on nicotine dependence. Journal of Human Genetics 49: 35-39.

Lingjaerde, O. 1969. Uptake of serotonin in blood platelets: Dependence on sodium and chloride, and inhibition by choline. FEBS Letters 3: 103-106.

Little, K.Y., Elmer, L.W., Zhong, H., Scheys, J.O., and Zhang, L. 2002. Cocaine induction of dopamine transporter trafficking to the plasma membrane. Molecular Pharmacology 61: 436-445.

Liu-Chen, L.Y. 2004. Agonist-induced regulation and trafficking of kappa opioid receptors. Life Sciences 75: 511-536.

Lizunov, V.A., Matsumoto, H., Zimmerberg, J., Cushman, S.W., and Frolov, V.A. 2005. Insulin stimulates the halting, tethering, and fusion of mobile GLUT4 vesicles in rat adipose cells. Journal of Cell Biology 169: 481-489. 
Logrip, M.L., Janak, P.H., and Ron, D. 2009. Blockade of ethanol reward by the kappa opioid receptor agonist U50,488H. Alcohol 43: 359-365.

Lopez, J.A., Burchfield, J.G., Blair, D.H., Mele, K., Ng, Y., Vallotton, P., James, D.E., and Hughes, W.E. 2009. Identification of a distal GLUT4 trafficking event controlled by actin polymerization. Molecuar Biology of the Cell 20: 3918-3929.

Lyons, D.J., Horjales, A., E., and Broberger, C. 2010. Synchronized network oscillations in rat tuberoinfundibular dopamine neurons: switch to tonic discharge by thyrotropin-releasing hormone. Neuron 65: 217-229.

M.O.H. 2007. Drug use in New Zealand: analysis of the 2003 New Zealand health behaviours survey - drug use. Ministry of Health, Wellington.

Mague, S.D., Pliakas, A.M., Todtenkopf, M.S., Tomasiewicz, H.C., Zhang, Y., Stevens, W.C., Jones, R.M., Portoghese, P.S., and Carlezon, W.A. 2003. Antidepressantlike effects of kappa-opioid receptor antagonists in the forced swim test in rats. Journal of Pharmacology and Experimental Therapeutics 305: 323-330.

Maina, F.K., and Matthews, T.A. 2010. Functional fast scan cyclic voltammetry assay to characterize dopamine D2 and D3 autoreceptors in the mouse striatum. ACS Chemical Neuroscience 1: 450-462.

Maisonneuve, I.M., Archer, S., and Glick, S.D. 1994. U50,488, a kappa opioid receptor agonist, attenuates cocaine-induced increases in extracellular dopamine in the nucleus accumbens of rats. Neuroscience Letters 181: 57-60.

Maiya, R., Buck, K.J., Harris, A., and Mayfield, R.D. 2002. Ethanol-sensitive sites on the human dopamine transporter. Journal of Biological Chemistry 277: 3072430729.

Mananares, J., Lookingland, K.J., and Moore, K.R. 1991. Kappa opioid receptormediated regulation of dopaminergic neurons in the rat brain. The Journal of Pharmacology and Experimental Therapeutics 256: 500-505.

Mao, Y., Mathewson, L., Gesmonde, J., Sato, Y., Holy, M., Sitte, H.H., and Rudnick, G. 2000. Involvement of serotonin transporter extracellular loop 1 in serotonin binding and transport. Molecular Membrane Biology 25: 115-127.

Margolis, E.B., Hjelmstad, G.O., Bonci, A., and Fields, H.L. 2003. kappa-opioid agonists directly inhibit midbrain dopaminergic neurons. Journal of Neuroscience 23: 9981-9986.

Margolis, E.B., Lock, H., Chefer, V.I., Shippenberg, T.S., Hjelmstad, G.O., and Fields, H.L. 2006. kappa opioids selectively control dopaminergic neurons projecting to the prefrontal cortex. Proceedings of the National Academy of Sciences of the United States of America 103: 2938-2942.

Marinova, Z., Vukojevic, V., Surcheva, S., Yakovleva, T., Cebers, G., Pasikova, N., Usynin, I., Hugonin, L., Fang, W., Hallberg, M., et al. 2005. Translocation of dynorphin neuropeptides across the plamsa membrane. The Journal of Biological Chemistry 280: 26360-26370.

Mash, D.C., Pablo, J., Ouyang, Q., Hearn, W.L., and Izenwasser, S. 2002. Dopamine transport function is elevated in cocaine users. Journal of Neurochemistry 81: 292-300.

Mash, D.C., and Staley, J.K. 1999. $\mathrm{D}_{3}$ dopamine and kappa opioid receptor alternations in human brain of cocaine-overdose victims. Annals New York Academy of Sciences 877: 507-522.

Mason, J.N., Farmer, H., Tomlinson, I.D., Schwartz, J.W., Savchenko, V., DeFelice, L.J., Rosenthal, S.J., and Blakely, R.D. 2005. Novel fluorescence-based approaches for the study of biogenic amine transporter localization, activity, and regulation. Journal of Neuroscience Methods 143: 3-25. 
Mayfield, R.D., Maiya, R., Keller, D., and Zahniser, N.R. 2001. Ethanol potentiates the function of the human dopamine transporter expressed in Xenopus oocytes. Journal of Neurochemistry 79: 1070-1079.

Mazei, M.S., Pluto, C.P., Kirkbride, B., and Pehek, E.A. 2002. Effects of catecholamine uptake blockers in the caudate-putamen and subregions of the medial prefrontal cortex of the rat. Brain Research 936: 58-67.

McCurdy, C.R., Sufka, K.J., Smith, G.H., Warnick, J.E., and Nieto, M.J. 2006. Antinociceptive profile of salvinorin A, a structurally unique kappa opioid receptor agonist. Pharmacology Biochemistry and Behavior 83: 109-113.

McElvain, J.S., and Schenk, J.O. 1992. A multisubstrate mechanism of striatal dopamine uptake and its inhibition by cocaine. Biochemical Pharmacology 43: 2189-2199.

McLaughlin, J.P., Land, B.B., Li, S., Pintar, J.E., and Chavkin, C. 2006a. Prior activation of kappa opioid receptors by U50,488 mimics repeated forced swim stress to potentiate cocaine place preference conditioning. Neuropsychopharmacology 31: 787-794.

McLaughlin, J.P., Li, S., Valdez, J., Chavkin, T.A., and Chavkin, C. 2006b. Social defeat stress-induced behavioral responses are mediated by the endogenous kappa opioid system. Neuropsychopharmacology 31: 1241-1248.

McLaughlin, J.P., Marton-Popovici, M., and Chavkin, C. 2003a. kappa opioid receptor antagonism and prodynorphin gene disruption block stress-induced behavioral responses. Journal of Neuroscience 23: 5674-5683.

McLaughlin, J.P., Xu, M., Mackie, K., and Chavkin, C. 2003b. Phosphorylation of a carboxyl-terminal serine within the $\kappa$-opioid receptor produces desensitization and internalization. The Journal of Biological Chemistry 278: 34631-34640.

McLennan, G.P., Kiss, A., Miyatake, M., Belcheva, M.M., Chambers, K.T., Pozek, J.J., Mohabbat, Y., Moyer, R.A., Bohn, L.M., and Coscia, C.J. 2008. Kappa opioids promote the proliferation of astrocytes via $\mathrm{G}$ beta gamma and beta-arrestin 2dependent MAPK-mediated pathways. Journal of Neurochemistry 107: 17531765.

Medana, C., Massolino, C., Pazzi, M., and Biacchi, C. 2006. Determination of salvinorins and divinatorins in Salvia divinorum leaves by liquid chromatography/multistage mass spectrometry. Rapid Communications in Mass Spectrometry 20: 131-136.

Mehler-Wex, C., Riederer, P., and Gerlach, M. 2006. Dopaminergic dysbalance in distinct basal ganglia neurocircuits: implications for the pathophysiology of Parkinson's disease, schizophrenia and attention deficit hyperactivity disorder. Neurotoxicity Research 10: 167-179.

Meiergerd, S.M., Hooks, S.M., and Schenk, J.O. 1994a. The striatal transporter for dopamine in the rat may be kinetically up-regulated following 3 weeks of withdrawal from cocaine self-administration. Journal of Neurochemistry 63: 1277-1281.

Meiergerd, S.M., McElvain, J.S., and Schenk, J.O. 1994b. Effects of cocaine and repeated cocaine followed by withdrawal: alterations of dopaminergic transporter turnover with no changes in kinetics of substrate recognition. Biochemical Pharmacology 47: 1627-1634.

Meiergerd, S.M., Patterson, T.A., and Schenk, J.O. 1993. $\mathrm{D}_{2}$ receptors may modulate the function of the striatal transporter for dopamine: kinetic evidence from studies in vitro and in vivo. Journal of Neurochemistry 61: 764-767.

Meiergerd, S.M., and Schenk, J.O. 1995. Measurement of the time-resolved kinetics of biogenic amine release and transporter activity by rotating disk electrode voltammetry in vitro. In Neuromethods, Vol. 27: Voltammetric Methods in Brain 
Systems. (eds. A. Boulton, G. Baker, and R.N. Adams), pp. 305-337. Humana Press Inc, USA.

Melikian, H.E., and Buckley, K.M. 1999. Membrane trafficking regulates the activity of the human dopamine transporter. The Journal of Neuroscience 19: 7699-7710.

Mello, N.K., and Negus, S.S. 1998. Effects of kappa opioid agonists on cocaine- and food-maintained responding by rhesus monkeys. Journal of Pharmacology and Experimental Therapeutics 286: 812-824.

Merg, F., Filliol, D., Usynin, I., Bazov, I., Bark, N., Hurd, Y.L., Yakovleva, T., Kieffer, B.L., and Bakalkin, G. 2006. Big dynorphin as a putative endogenous ligand for the $\kappa$-opioid receptor. Journal of Neurochemistry 97: 292-301.

Meshul, C.K., and McGinty, J.F. 2000. Kappa opioid receptor immunoreactivity in the nucleus accumbens and caudate-putamen is primarily associated with synaptic vesicles in axons. Neuroscience 96: 91-99.

Middleton, L.S., Apparsundaram, S., King-Pospisil, K.A., and Dwoskin, L.P. 2007. Nicotine increases dopamine transporter function in rat striatum through a trafficking-independent mechanism. European Journal of Pharmacology 554: 128-136.

Middleton, L.S., Cass, W.A., and Dwoskin, L.P. 2004. Nicotinic receptor modulation of dopamine transporter function in rat striatum and medial prefrontal cortex. Journal of Pharmacology and Experimental Therapeutics 308: 367-377.

Miner, L.H., Schroeter, S., Blakely, R.D., and Sesack, S.R. 2000. Ultrastructural localization of the serotonin transporter in the superficial and deep layers of the rat prelimbic prefrontal cortex and its spatial relationship to dopamine terminals. The Journal of Comparative Neurology 427: 220-234.

Miranda, M., Dionne, K.R., Sorkina, T., and Sorkin, A. 2007. Three ubiquitin conjugation sites in the amino terminus of the dopamine transporter mediate protein kinase C-dependent endocytosis of the transporter. Molecuar Biology of the Cell 18: 313-323.

Miranda, M., Wu, C.C., Sorkina, T., Korstjens, D.R., and Sorkin, A. 2005. Enhanced ubiquitylation and accelerated degradation of the dopamine transporter mediated by protein kinase C. The Journal of Biological Chemistry 280: 35617-35624.

Mochizuki, H., Amano, T., Seki, T., Matsubayashi, H., Mitsuhata, C., Morita, K., Kitayama, S., Dohi, T., Mishima, H.K., and Sakai, N. 2005. Role of C-terminal region in the functional regulation of rat serotonin transporter (SERT). Neurochemistry International 46: 93-105.

Morani, A.S., Kivell, B., Prisinzano, T.E., and Schenk, S. 2009. Effect of kappa-opioid receptor agonists U69593, U50488H, spiradoline and salvinorin A on cocaineinduced drug-seeking in rats. Pharmacology Biochemistry and Behavior 94: 244-249.

Moron, J.A., Zakharova, I., Ferrer, J.V., Merrill, G.A., Hope, B., Lafer, E.M., Lin, Z.C., Wang, J.B., Javitch, J.A., Galli, A., et al. 2003. Mitogen-activated protein kinase regulates dopamine transporter surface expression and dopamine transport capacity. Journal of Neuroscience 23: 8480-8488.

Mortensen, O.V., and Amara, S.G. 2003. Dynamic regulation of the dopamine transporter. European Journal of Pharmacology 479: 159-170.

Mowry, M., Mosher, M., and Briner, W. 2003. Acute physiologic and chronic histologic changes in rats and mice exposed to the unique hallucinogen salvinorin A. Journal of Psychoactive Drugs 35: 379-382.

Muller, C.P., Carey, R.J., Salloum, J.B., and Huston, J.P. 2003. Serotonin1A-receptor agonism attenuates the cocaine-induced increase in serotonin levels in the hippocampus and nucleus accumbens but poteniates hyperlocomotion: an invivo microdialysis study. Neuropharmacology 44: 592-603. 
Munro, T.A., Rizzacasa, M.A., Roth, B.L., Toth, B.A., and Yan, F. 2005. Studies toward the pharmacophore of salvinorin A, a potent kappa opioid receptor agonist. Journal of Medicinal Chemistry 48: 345-348.

Murphy, D.L., Fox, M.A., Timpano, K.R., Moya, P.R., Ren-Patterson, R., Andrews, A.M., Holmes, A., Lesch, K.P., and Wendland, J.R. 2008. How the serotonin story is being rewritten by new gene-based discoveries principally related to SLC6A4, the serotonin transporter gene, which functions to influence all cellular serotonin systems. Neuropharmacology 55: 932-960.

Navailles, S., De Deurwaerdere, P., Porras, G., and Spampinato, U. 2004. In vivo evidence that $5-\mathrm{HT}_{2 \mathrm{C}}$ receptor antagonist but not agonist modulates cocaineinduced dopamine overflow in the rat nucleus accumbens and striatum. Neuropsychopharmacology 29: 319-326.

Near, J.A., Bigelow, J.C., and Wightman, R.M. 1988. Comparison of uptake of doamine in rat striatal chopped tissue and synaptosomes. The Journal of Pharmacology and Experimental Therapeutics 245: 921-927.

Negus, S.S., Mello, N.K., Linsenmayer, D.C., Jones, R.M., and Portoghese, P.S. 2002. Kappa opioid antagonist effects of the novel kappa antagonist 5'guanidinonaltrindole (GNTI) in an assay of schedule-controlled behavior in rhesus monkeys. Psychopharmacology 163: 412-419.

Negus, S.S., Mello, N.K., Portoghese, P.S., and Lin, C.E. 1997. Effects of kappa opioids on cocaine self-administration by rhesus monkeys. Journal of Pharmacology and Experimental Therapeutics 282: 44-55.

Nemeth, C., Paine, T.A., Rittiner, J.E., Beguin, C., Carroll, F.I., Roth, B.L., Cohen, B.M., and Carlezon, W.A.J. 2010. Role of kappa-opioid receptors in the effects of salvinorin A and ketamine on attention in rats. Psychopharmacology 210: 263-274.

Nestler, E.J., and Aghajanian, G.K. 1997. Molecular and cellular basis of addiction. Science 278: 58-63.

Neville, M.J., Johnstone, E.C., and Walton, R.T. 2004. Identification and characterization of ANKK1: a novel kinase gene closely linked to DRD2 on chromosome band 11q23.1. Human Mutation 23: 540-545.

Nirenberg, M.J., Chan, J., Pohorille, A., Vaughan, R.A., Uhl, G.R., Kuhar, M.J., and Pickel, V.M. 1997. The dopamine transporter: comparative ultrastructure of dopaminergic axons in limbic and motor compartments of the nucleus accumbens. The Journal of Neuroscience 17: 6899-6907.

Nishimura, S.Y., Vrljic, M., Klein, L.O., McConnell, H.M., and Moerner, W.E. 2006. Cholesterol depletion induces solid-like regions in the plasma membrane. Biophysical Journal 90: 927-938.

Noble, E.P., Blum, K., Khalsa, M.E., Ritchie, T.L., Montgomery, A., Wood, R.C., Fitch, R.J., Ozkaragoz, T., Sheridan, P.J., Anglin, M.D., et al. 1993. Allelic association of the $\mathrm{D}_{2}$ dopamine receptor gene with cocaine dependence. Drug and Alcohol Dependence 33.

Noble, E.P., Zhang, X., Ritchie, T.L., and Sparkes, R.S. 2000. Haplotypes at the DRD2 locus and severe alcoholism. American Journal of Medical Genetics (Neuropsychiatric Genetics) 96: 622-631.

Nozawa, M., Suka, Y., Hoshi, T., Suzuki, T., and Hagiwara, H. 2008. Total synthesis of the hallucinogen neoclerodane diterpenoid salvinorin A. Organic Letters 10: 1365-1368.

O'Connell, K.M.S., and Tamkun, M.M. 2005. Targeting of voltage-gated potassium channel isoforms to distinct cell surface microdomains. Journal of Cell science 118: $2155-2166$. 
O'Hara, C.M., Uhland-Smith, A., O'Malley, K.L., and Todd, R.D. 1996. Inhibition of dopamine synthesis by dopamine D2 and D3 but not D4 receptors. Journal of Pharmacology and Experimental Therapeutics 277: 186-192.

Ohno, M., Yamamoto, T., and Ueki, S. 1992. Analgesic and discriminative stimulus properties of U-62,066E, the selective kappa-opioid receptor agonist, in the rat. Psychopharmacology 106: 31-38.

Ohyama, K., Sogawa, C., Sogawa, N., Morita, K., Dohi, T., and Kitayama, S. 2010. Nicotine stimulates transcriptional activity of the human dopamine transporter gene. Neuroscience Letters 471: 34-37.

Oz, M., Jaligam, V., Galadari, S., Petroianu, G., Shuba, Y.M., and Shippenberg, T.S. 2010a. The endogenous cannabinoid, anandamide, inhibits dopamine transporter function by a receptor-independent mechanism. Journal of Neurochemistry 112: 1454-1464.

Oz, M., Libby, T., Kivell, B., Jaligam, V., Ramamoorthy, S., and Shippenberg, T.S. 2010b. Real-time, spatially resolved analysis of serotonin transporter activity and regulation using the fluorescent substrate, ASP+. Journal of Neurochemistry 114: 1019-1029.

Page, G., Barc-Pain, S., Pontcharraud, R., Cante, A., Piriou, A., and Barrier, L. 2004. The up-regulation of the striatal dopamine transporters' activity by cAMP is PKA-, CaMK II- and phosphate-dependent. Neurochemistry International 45: 627-632.

Patel, A., Uhl, G.R., and Kuhar, M.J. 1993. Species differences in dopamine transporters: postmortem changes and glycosylation differences. Journal of Neurochemistry 61: 496-500.

Perreault, M.L., Dawn, G., Scattolon, S., Wang, Y., Szechtma, H., and Foster, J.D. 2007. Cotreatment with the kappa opioid agonist U69593 enhances locomotor sensitization to the D2/D3 dopamine agonist quinpirole and alters dopamine D2 receptor and prodynorphin mRNA expression in rats. Psychopharmacology 194: 485-496.

Perreault, M.L., Graham, D., Bisnaire, L., Simms, J., Hayton, S., and Szechtman, H. 2006. Kappa-opioid agonist U69593 potentiates locomotor sensitization to the D2/D3 agonist quinpirole: pre- and postsynaptic mechanisms. Neuropsychopharmacology 31: 1967-1981.

Perry, S.W., Barbieri, J., Tong, N., Polesskaya, O., Pudasaini, S., Stout, A., Lu, R., Kiebala, M., Maggirwar, S.B., and Gelbard, H.A. 2010. Human immunodeficiency virus-1 tat activates calpain proteases via the ryanodine receptor to enhance surface dopamine transporter levels and increase transporter-specific uptake and $\mathrm{V}_{\max }$. The Journal of Neuroscience 30: 1415314164.

Persico, A.M., Bird, G., Gabbay, F.H., and Uhl, G.R. 1996. $\mathrm{D}_{2}$ dopamine receptor gene TaqI A1 and B1 restriction fragment length polymorphisms: enhanced frequencies in psychostimulant-preferring polysubstance abusers. Biological Psychiatry 40: 776-784.

Pichini, S., Abanades, S., Farre, M., Pellegrini, M., Marchei, E., Pacifici, R., de la Torre, R., and Zuccaro, P. 2005. Quantification of the plant-derived hallucinogen Salvinorin A in conventional and non-conventional biological fluids by gas chromatography/mass spectrometry after Salvia divinorum smoking. Rapid Communications in Mass Spectrometry 19: 1649-1656.

Pickel, V.M., and Chan, J. 1996. Ultrastructural localization of the serotonin transporter in limbic and motor compartments of the nucleus accumbens. The Journal of Neuroscience 19: 7356-7366. 
Pike, L.J. 2003. Lipid rafts: Bringing order to chaos. Journal of Lipid Research 44: 655667.

Pizza, M., Bartoloni, A., Prugnola, A., Siverstri, S., and Rappuoli, R. 1988. Subunit S1 of pertussis toxin: mapping of the regions essential for ADP-ribosyltransferase activity. Proceedings of the National Academy of Sciences of the United States of America 85: 7521-7525.

Pohjalainen, T., Rinne, J.O., Nagren, K., Lehikoinen, P., Anttila, K., Syvalahti, E.K.G., and Heitala, J. 1998. The A1 allele of the human $\mathrm{D}_{2}$ dopamine receptor gene predicts low $\mathrm{D}_{2}$ receptor availability in healthy volunteers. Molecular Psychiatry 3: $256-260$.

Portoghese, P.S., Lipkowski, A.W., and Takemori, A.E. 1987. Binaltorphimine and norbinaltorphimine, potent and selective $\kappa$-opioid receptor antagonists. Life Sciences 40: 1287-1292.

Povlock, S.L., and Schenk, J.O. 1997. A multisubstrate kinetic mechanism of dopamine transport in the nucleus accumbens and its inhibition by cocaine. Journal of Neurochemistry 69: 1093-1105.

Preston, K.L., Umbricht, A., Schroeder, J.R., Abreu, M.E., Epstein, D.H., and Pickworth, W.B. 2004. Cyclazocine: comparison to hydromorphone and interaction with cocaine. Behavioural Pharmacology 15: 91-102.

Prisinzano, T.E., Tidgewell, K., and Harding, W.W. 2005. kappa opioids as potential treatments for stimulant dependence. Aaps Journal 7: E592-E599.

Pristupa, Z.B., McConkey, F., Liu, F., Man, H.Y., Lee, F.J.S., Wang, Y.T., and Niznik, H.B. 1998. Protein kinase-mediated bidirectional trafficking and functional regulation of the human dopamine transporter. Synapse 30: 79-87.

Przekop, P.D.O., Lee, T., and Linda, L. 2009. Persistent psychosis associated with Salvia divinorum use. American Journal of Psychiatry 166: 832.

Ramamoorthy, S., Bauman, A.L., Moore, K.R., Han, H., Yang-Feng, T., Chang, A.S., Ganapathy, V., and Blakely, R.D. 1993. Antidepressant- and cocaine-sensitive human serotonin transporter: molecular cloning, expression, and chromosomal location. Proceedings of the National Academy of Sciences of the United States of America 90: 2542-2546.

Ramamoorthy, S., and Blakely, R.D. 1999. Phosphorylation and sequestration of serotonin transporters differentially modulated by psychostimulants. Science 285: 763-766.

Ramamoorthy, S., Cool, D.R., Leibach, F.H., Mahesh, V.B., and Ganapathy, V. 1992. Reconstitution of the human placental 5-hydroxytrypamine transporter in a catalytically active form after detergent solubilization. Biochemical Journal 286: 89-95.

Ramamoorthy, S., Samuvel, D.J., and Jayanthi, L.D. 2002a. kappa-opioid receptor mediated regulation of serotonin transporter function in striatum. Society for Neuroscience Abstract Viewer and Itinerary Planner 2002: Abstract No. 442.442 .

Ramamoorthy, S., Samuvel, D.J., and Jayanthi, L.D. 2002b. Kappa-opioid receptor mediated regulation of serotonin transporter function in striatum. In 32nd Annual Meeting of the Society for Neuroscience, pp. 442.442, Orlando, Florida, USA.

Redila, V.A., and Chavkin, C. 2008. Stress-induced reinstatement of cocaine seeking is mediated by the kappa opioid system. Psychopharmacology 200: 59-70.

Richards, T.L., and Zahniser, N.R. 2009. Rapid substrate-induced down-regulation in function and surface localization of dopamine transporters: rat dorsal striatum versus nucleus accumbens. Journal of Neurochemistry 108: 1575-1584. 
Robinson, D.L., Volz, T.J., Schenk, J.O., and Wightman, R.M. 2005. Acute ethanol decreases dopamine transporter velocity in rat striatum: in vivo and in vitro electrochemical measurements. Alcoholism-Clinical and Experimental Research 29: 746-755.

Robinson, T.E., and Berridge, K.C. 1993. The neural basis of drug craving: an incentive-sensitization theory of addiction. Brain Research Reviews 18: 247291.

Robinson, T.E., and Berridge, K.C. 2000. The psychology and neurobiology of addiction: an incentive-sensitization view. Addiction 95: S91-S117.

Robinson, T.E., and Berridge, K.C. 2001. Incentive-sensitization and addiction. Addiction 96: 103-114.

Roitman, M.F., Patterson, T.A., Sakai, R.R., Bernstein, I.L., and Figlewicz, D.P. 1999. Sodium depletion and aldosterone decrease dopamine transporter activity in nucleus accumbens but not striatum. American Journal of PhysiologyRegulatory Integrative and Comparative Physiology 276: R1339-R1345.

Roth, B.L., Baner, K., Westkaemper, R., Siebert, D., Rice, K.C., Steinberg, S., Ernsberger, P., and Rothman, R.B. 2002. Salvinorin A: a potent naturally occurring nonnitrogenous kappa opioid selective agonist. Proceedings of the National Academy of Sciences of the United States of America 99: 11934-11939.

Rothblat, D.S., Rubin, E., and Schneider, J.S. 2001. Effects of chronic alcohol ingestion on the mesostriatal dopamine system in the rat. Neuroscience Letters 300: 6366.

Rothman, R.B., Murphy, D.L., Xu, H., Godin, J.A., Dersch, C.M., Partilla, J.S., Tidgewell, K., Schmidt, M., and Prisinzano, T.E. 2007. Salvinorin A: allosteric interactions at the mu-opioid receptor. Journal of Pharmacology and Experimental Therapeutics 320: 801-810.

Ruedi-Bettschen, D., Rowlett, J.K., Spealman, R.D., and Platt, D.M. 2010. Attenuation of cocaine-induced reinstatement of drug seeking in squirrel monkeys: kappa opioid and serotonergic mechanisms. Psychopharmacology 210: 169-177.

Rusin, K.I., Giovannucci, D.R., Stuenkel, E.L., and Moises, H.C. 1997. א-opioid receptor activation modulates $\mathrm{Ca}^{2+}$ currents and secretion in isolated neuroendocrine nerve terminals. Journal of Neuroscience 17: 6565-6574.

Rutter, J.J., and Auerbach, S.B. 1993. Acute uptake inhibition increases extracellular serotonin in the rat forebrain. Journal of Pharmacology and Experimental Therapeutics 265: 1319-1324.

Sabol, S.Z., Hu, S., and Hamer, D. 1998. A functional polymorphism in the monoamine oxidase A gene promoter. Human Genetics 103: 273-279.

Samuvel, D.J., Jayanthi, L.D., Bhat, N.R., and Ramamoorthy, S. 2005. A role for p38 mitogen-activated protein kinase in the regulation of the serotonin transporter: evidence for distinct cellular mechanisms involved in transporter surface expression. The Journal of Neuroscience 25: 29-41.

Saunders, C., Ferrer, J.V., Shi, L., Chen, J.Y., Merrill, G., Lamb, M.E., Leeb-Lundberg, L.M.F., Carvelli, L., Javitch, J.A., and Galli, A. 2000. Amphetamine-induced loss of human dopamine transporter activity: An internalization-dependent and cocaine-sensitive mechanism. Proceedings of the National Academy of Sciences of the United States of America 97: 6850-6855.

Scheerer, J.R., Lawrence, J.F., Wang, G.C., and Evans, D.A. 2007. Asymmetric synthesis of salvinorin A, a potent $\kappa$ opioid receptor agonist. Journal of the American Chemical Society 129: 8968-8969.

Scheffel, U., Pogun, S., Stathis, M., Boja, J.W., and Kuhar, M.J. 1991. In vivo labeling of cocaine binding sites on dopamine transporters with $\left[{ }^{3} \mathrm{H}\right]$ WIN 35,428 . The Journal of Pharmacology and Experimental Therapeutics 257: 954-958. 
Schenk, S., and Partridge, B. 2001. Effect of the kappa-opioid receptor agonist, U69593, on reinstatement of extinguished amphetamine self-administration behavior. Pharmacology Biochemistry and Behavior 68: 629-634.

Schenk, S., Partridge, B., and Shippenberg, T.S. 1999. U69593, a kappa-opioid agonist, decreases cocaine self-administration and decreases cocaine-produced drugseeking. Psychopharmacology 144: 339-346.

Schenk, S., Partridge, B., and Shippenberg, T.S. 2000. Reinstatement of extinguished drug-taking behavior in rats: effect of the kappa-opioid receptor agonist, U69593. Psychopharmacology 151: 85-90.

Schlosser, B., Kudernatsch, M.B., Sutor, B., and Bruggencate, G.t. 1995. $\delta, \mu$ and $\kappa$ opioid receptor agonists inhibit dopamine overflow in rat neostriatal slices. Neuroscience Letters 191: 126-130.

Schmidt, M., Schmidt, M., Butelman, E.R., Harding, W.W., Tidgewell, K., Murry, D.J., Kreek, M.J., and Prisinzano, T.E. 2005a. Pharmacokinetics of the plant-derived א-opioid hallucinogen salvinorin A in nonhuman primates. Synapse 58: 208210.

Schmidt, M.S., Prisinzano, T.E., Tidgewell, K., Harding, W.W., Butelman, E.R., and Kreek, M.J. 2005b. Determination of salvinorin A in body fluids by high performance liquid chromatography - atmospheric pressure chemical ionization. Journal of Chromatography 818: 221-225.

Scholze, P., Zwach, J., Kattinger, A., Pifl, C., Singer, E.A., and Sitte, H.H. 2000. Transporter-mediated release: a superfusion study on human embryonic kidney cells stably expressing the human serotonin transporter. The Journal of Pharmacology and Experimental Therapeutics 293: 870-878.

Schwartz, J.W., Blakely, R.D., and DeFelice, L.J. 2003. Binding and transport in norepinephrine transporters - Real-time, spatially resolved analysis in single cells using a fluorescent substrate. Journal of Biological Chemistry 278: 97689777.

Schwartz, J.W., Novarino, G., Piston, D.W., and DeFelice, L.J. 2005. Substrate binding stoichiometry and kinetics of the norepinephrine transporter. Journal of Biological Chemistry 280: 19177-19184.

Seeman, P., Guan, H.-C., and Hirbec, H. 2009. Dopamine D $2^{\text {High }}$ receptors stimulated by phencyclidines, lysergic acid diethylamine, salvinorin $\mathrm{A}$, and modafinil. Synapse 63: 698-704.

Sesack, S.R., Hawrylak, V.A., Matus, C., Guido, M., and Levey, A.I. 1998. Dopamine axon varicosities in the prelimbic division of the rat prefrontal cortex exhibit sparse immunoreactivity for the dopamine transporter. The Journal of Neuroscience 18: 2697-2708.

Shaw, G., Morse, S., Ararat, M., and Graham, F.L. 2002. Preferential transformation of human neuronal cells by human adenoviruses and the origin of HEK 293 cells. The FASEB Journal 16: 869-871.

Shearer, J., Darke, S., Rodgers, C., Slade, T., van Beek, I., Lewis, J., Brady, D., McKetin, R., Mattick, R.P., and Wodak, A. 2009. A double-blind, placebocontrolled trial of modafinil (200 mg/day)) for methamphetamine dependence. Addiction 104: 224-233.

Sheffler, D.J., and Roth, B.L. 2003. Salvinorin A: the 'magic mint' hallucinogen finds a molecular target in the kappa opioid receptor. Trends in Pharmacological Sciences 24: 107-109.

Shi, J., Zhao, L.-Y., Copersino, M.L., Fang, Y.-X., Chen, Y., Tian, J., Deng, Y., Shuai, Y., Jin, J., and Lu, L. 2008a. PET imaging of dopamine transporter and drug craving during methadone maintenance treatment and after prolonged abstinence in heroin users. European Journal of Pharmacology 579: 160-166. 
Shi, L., Quick, M., Zhao, Y., Weinstein, H., and Javitch, J.A. 2008b. The mechanism of a neurotransmitter: sodium symporter - inward release of $\mathrm{Na}^{+}$and substrate is triggered by substrate in a second binding site. Molecular Cell 30: 667-677.

Shields, P.G., Lerman, C., Audrain, J., Bowman, E.D., Main, D., Boyd, N.R., and Caporaso, N.E. 1998. Dopamine D4 receptors and the risk of cigarette smoking in african-americans and caucasians. Cancer Epidemiology, Biomarkers \& Prevention 7: 453-458.

Shimada, S., Kitayama, S., Lin, C.-L., Patel, A., Nanthakumar, E., Gregor, P., Kuhar, M.J., and Uhl, G.R. 1991. Cloning and expression of a cocaine-sensitive rat dopamine transporter complementary DNA. Science 254: 576-578.

Shippenberg, T.S., and Rea, W. 1997. Sensitization to the behavioral effects of cocaine: modulation by dynorphin and $\kappa$-opioid receptor agonists. Pharmacology Biochemistry and Behavior 57: 449-455.

Shippenberg, T.S., Zapata, A., and Chefer, V.I. 2007. Dynorphin and the pathophysiology of drug addiction. Pharmacology \& Therapeutics 116: 306321.

Siebert, D.J. 1994. Salvia-Divinorum and salvinorin A - new pharmacological findings. Journal of Ethnopharmacology 43: 53-56.

Simantov, R. 1993. Chronic morphine alters dopamine transporter density in the rat brain: possible role in the mechanism of drug addiction. Neuroscience Letters 163: $121-124$.

Slack, A., Nana, G., Webster, M., Stokes, F., and Wu, J. 2009. Costs of harmful alcohol and other drug use. (ed. BERL). Business and Economic Research Limited, Wellington.

Snapp, E.L., Altan, N., and Lippincott-Schwartz, J. 2003. Measuring protein mobility by photobleaching GFP chimeras in living cells. In Current Protocols in Cell Biology, pp. Unit 21.21.

Soeffing, J.M., Martin, L.D., Fingerhood, M.I., Jasinski, D.R., and Rastegar, D.A. 2009. Buprenorphine maitenance treatment in a primary care setting: outcomes at 1 year. Journal of Substance Abuse Treatment 37: 426-430.

Solomon, R.L., and Corbit, J.D. 1974. An oppenent-process theory of motivation: 1. temoporal dynamics of affect. Pyschological Review 81: 119-145.

Sonders, M.S., Zhu, S.-J., Zahniser, N.R., Kavanaugh, M.P., and Amara, S.G. 1997. Multiple ionic conductances of the human dopamine transporter: the actions of dopamine and psychostimulants. The Journal of Neuroscience 17: 960-974.

Spitz, M.R., Shi, H., Yang, F., Hudmon, K.S., Jiang, H., Chamberlain, R.M., Amos, C.I., Wan, Y., Cinciripini, P., Hong, W.K., et al. 1998. Case-control study of the D2 dopamine receptor gene and smoking status in lung cancer patients. Journal of the National Cancer Institute 90: 358-363.

Srisurapanont, M., and Jarusuraisin, N. 2005. Naltrexone for the treatment of alcoholism: a meta-analysis of randomized controlled trials. International Journal of Neuropsychopharmacology 8: 267-280.

Steiner, H., and Gerfen, C.R. 1996. Dynorphin regulates D1 dopamine receptormediated responses in the striatum: relative contributions of pre- and postsynaptic mechanisms in dorsal and ventral striatum demonstrated by altered immediate-early gene induction. The Journal of Comparative Neurology 376: 530-541.

Stenstrom, A., Oreland, L., Hardy, J., Wester, P., and Winblad, B. 1985. The uptake of serotonin and dopamine by homogenates of frozen rat and human brain tissue. Neurochemical Research 10: 591-599. 
Steyn, F.J., Anderson, G.M., and Grattan, D.R. 2007. Expression of ovarian steroid hormone receptors in tuberoinfundibular dopaminergic neurones during pregnancy and lactation. Journal of Neuroendocrinology 19: 788-793.

Sumnall, H.R., Measham, F., Brandt, S.D., and Cole, J.C. 2010. Salvia divinorum use and phenomenology: results from an online survey. Journal of Psychopharmacology doi: 10.1177/0269881110385596

Sun, W., Xue, Y., Huang, Z., and Steketee, J.D. 2010. Regulation of cocaine-reinstated drug-seeking behavior by $\kappa$-opioid receptors in the ventral tegmental area of rats. Psychopharmacology 210: 179-188.

Sur, C., Betz, H., and Schloss, P. 1996. Immunocytochemical detection of the serotonin transporter in rat brain. Neuroscience 73: 217-231.

Svingos, A.L., Chavkin, C., Colago, E.E.O., and Pickel, V.M. 2001. Major coexpression of kappa-opioid receptors and the dopamine transporter in nucleus accumbens axonal profiles. Synapse 42: 185-192.

Tang, L., Todd, R.D., and O'Malley, K.L. 1994. Dopamine D2 and D3 receptors inhibit dopamine release. Journal of Pharmacology and Experimental Therapeutics 270: $475-479$.

Tao, R., and Auerbach, S.B. 2002. Opioid receptor subtypes differentially modulate serotonin efflux in the rat central nervous system. Journal of Pharmacology and Experimental Therapeutics 303: 549-556.

Tardieu, S., Poirier, Y., Micallef, J., and Blin, O. 2004. Amphetamine-like stimulant cessation in an abusing patient treated with bupropion. Acta Psychiatrica Scandinavica 109: 77-78.

Teksin, Z.S., Lee, I.J., Nemieboka, N.N., Othman, A.A., Upreti, V.V., Hassem, H.E., Syed, S.S., Prisinzano, T.E., and Eddington, N.D. 2009. Evaluation of the transport, in vitro metabolism and pharmacokinetics of salvinorin A, a potent hallucinogen. European Journal of Pharmaceutics and Biopharmaceutics 72: 471-477.

Thomas, J.B., Atkinson, R.N., Rothman, R.B., Fix, S.E., Mascarella, S.W., Vinson, N.A., Xu, H., Dersch, C.M., Lu, Y.-F., Cantrell, B.E., et al. 2001. Identification of the first trans-(3R,4R)-dimethyl-4-(3-hydroxyphenyl)piperidine derivative to possess highly potent and selective opioid $\kappa$ receptor antagonist activity. Journal of Medicinal Chemistry 44: 2687-2690.

Thompson, A.C., Zapata, A., Justice, J.B., Vaughan, R.A., Sharpe, L.G., and Shippenberg, T.S. 2000. kappa-opioid receptor activation modifies dopamine uptake in the nucleus accumbens and opposes the effects of cocaine. Journal of Neuroscience 20: 9333-9340.

Tiihonen, J., Hallikainen, T., Lachman, H.M., Saito, T., Volavka, J., Kauhanen, J., Salonen, J.T., Ryynanen, O.-P., Pohjalainen, T., Syvalahti, E.K.G., et al. 1999. Association between the functional variant of the catechol-O-methyltransferase (COMT) gene and type 1 alcoholism. Molecular Psychiatry 4: 286-289.

Tiihonen, J., Kuoppasalmi, K., Fohr, J., Tuomola, P., Kuikanmaki, O., Vorma, H., Sokero, P., Haukka, J., and Meririnne, E. 2007. A comparison of aripiprazole, methylphenidate, and placebo for amphetamine dependence. The American Journal of Psychiatry 164: 160-162.

Tomasiewicz, H.C., Todtenkoph, M.S., Chartoff, E.H., Cohen, B.M., and Carlezon, W.A.J. 2008. The kappa-opioid agonist U69,593 blocks cocaine-induced enhancement of brain stimulation reward. Biological Psychiatry 64: 982-988.

Torres, G.E., Carneiro, A., Seamans, K., Fiorentini, C., Sweeney, A., Yao, W.D., and Caron, M.G. 2003. Oligomerization and trafficking of the human dopamine transporter - mutational analysis identifies critical domains important for the 
functional expression of the transporter. Journal of Biological Chemistry 278: 2731-2739.

Tsujikawa, K., Kuwayama, K., Miyaguchi, H., Kanamori, T., Iwata, Y.T., and Inoue, H. 2009. In vitro stability and metabolism of salvinorin $\mathrm{A}$ in rat plasma. Xenobiotica 39: 391-398.

Tsuruda, P.R., Yung, J., Martin, W.J., Chang, R., Mai, N., and Smith, J.A.M. 2010. Influence of ligand binding kinetics on functional inhibition of human recombinant serotonin and norepinephrine transporters. Journal of Pharmacological and Toxicological Methods 61: 192-204.

Turchan, J., Przewlocka, B., Lason, W., and Przewlocki, R. 1998. Effects of repeated psychostimulant administration on the prodynorphin system activity and kappa opioid receptor density in the rat brain. Neuroscience 85: 1051-1059.

Tzaferis, J.A., and McGinty, J.F. 2001. Kappa opioid receptor stimulation decreases amphetamine-induced behavior and neuropeptide mRNA expression in the striatum. Molecular Brain research 93: 27-35.

Unterwald, E.M., Rubenfeld, J.M., and Kreek, M.J. 1994. Repeated cocaine administration upregulates $\kappa$ and $\mu$ but not $\delta$, opioid receptors. NeuroReport 5: 1613-1616.

Valdes, L.J. 1994. Salvia divinorum and the unique diterpene hallucinogen, salvinorin (divinorin)-A. Journal of Psychoactive Drugs 26: 277-283.

Valdes, L.J., Diaz, J.L., and Paul, A.G. 1983. Ethnopharmacology of ska Maria Pastora (salvia divinorum, epling and Jativa-M). Journal of Ethnopharmacology 7: 287312.

Valjent, E., Corvol, J.-C., Pages, C., Besson, M.-J., Maldonado, R., and Caboche, J. 2000. Involvement of the extracelllular signal-regulated kinase cascade for cocaine-rewarding properties. Journal of Neuroscience 20: 8701-8709.

Valjent, E., Pascoli, V., Svenningsson, P., Paul, S., Enslen, H., Corvol, J.-C., Stipanovich, A., Caboche, J., Lombroso, P.J., Nairn, A.C., et al. 2005. Regulation of a protein phosphatase cascade allows convergent dopamine and glutamate signals to activate ERK in the striatum. Proceedings of the National Academy of Sciences of the United States of America 102: 491-496.

Vandenbergh, D.J., Rodriguez, L.A., Miller, I.T., Uhl, G.R., and Lachman, H.M. 1997. High-activity catechol-O-methyltransferase allele is more prevalent in polysubstance abusers. American Journal of Medical Genetics (Neuropsychiatric Genetics) 74: 439-442.

Vanderschuren, L.J.M.J., Schoffelmeer, A.N.M., Wardeh, G., and De Vries, T.J. 2000. Dissociable effects of the k-opioid receptor agonists bremazocine, U69593, and U50488H on locomotor activity and long-term behavioral sensitization induced by amphetamine and cocaine. Psychopharmacology 150: 35-44.

Vanyukov, M.M., Moss, H.B., Yu, L.-M., Tarter, R.E., and Deka, R. 1995. Preliminary evidence for an association of a dinucleotide repeat polymorphism at the MAOA gene with early onset alcoholism/substance abuse. American Journal of Medical Genetics (Neuropsychiatric Genetics) 60: 122-126.

Vaughan, R.A., Huff, R.A., Uhl, G.R., and Kuhar, M.J. 1997. Protein kinase Cmediated phosphorylation and functional regulation of dopamine transporters in striatal synaptosomes. The Journal of Biological Chemistry 272: 15541-15546.

Venton, B.J., Seipel, A.T., Phillips, P.E.M., Wetsel, W.C., Gitler, D., Greengard, P., Augustine, G.J., and Wightman, R.M. 2006. Cocaine increases dopamine release by mobilization of a synapsin-dependent reserve pool. The Journal of Neuroscience 26: 3206-3209.

Vina-Vilaseca, A., and Sorkin, A. 2010. Lysine 63-linked polyubiquitination of the dopamine transporter requires WW3 and WW4 domains of Nedd4-2 and 
UBE2D ubiquitin-conjugating enzymes. The Journal of Biological Chemistry 285: 7645-7656.

Volkow, N.D., Wang, G.-J., Fowler, J.S., Logan, J., Hitzemann, R., Ding, Y.-S., Pappas, N., Shea, C., and Piscani, K. 1996. Decreases in dopamine receptors but not in dopamine transporters in alcoholics. Alcoholism: Clinical and Experimental Research 20: 1594-1598.

Volz, T.J., Farnsworth, S.J., Hanson, G.R., and Fleckenstein, A.E. 2009. Measurement of plasmalemmal dopamine transport, vesicular dopamine transport, and $\mathrm{K}^{+}$stimulated dopamine release in frozen rat brain tissue. Journal of Neuroscience Methods 180: 317-320.

Volz, T.J., Hanson, G.R., and Fleckenstein, A.E. 2006. Measurement of kinetically resolved vesicular dopamine uptake and efflux using rotating disk electrode voltammetry. Journal of Neuroscience Methods 155: 109-115.

Volz, T.J., and Schenk, J.O. 2005. A comprehensive atlas of the topography of functional groups of the dopamine transporter. Synapse 58: 72-94.

Von Voigtlander, P., and Lewis, R.A. 1982. U-50,488, a selective kappa opioid agonist: comparison to other reputed kappa agonists. Progress in NeuroPsychopharmacology \& Biological Psychiatry 6: 467-470.

Vrljic, M., Nishimura, S.Y., Moerner, W.E., and McConnell, H.M. 2005. Cholesterol depletion suppresses the translational diffusion of class II major histocompatibility complex proteins in the plasma membrane. Biophysical Journal 88: 334-347.

Walentiny, D.M., Vann, R.E., Warner, J.A., King, L.S., Seltzman, H.H., Navarro, H.A., Twine, C.E.J., Thomas, B.F., Gilliam, A.F., Gilmour, B.P., et al. 2010. Kappa opioid mediation of cannabinoid effects of the potent hallucinogen, salvinorin A, in rodents. Psychopharmacology 210: 275-284.

Walsh, S.L., Geter-Douglas, B., Strain, E.C., and Bigelow, G.E. 2001a. Enadoline and butorphanol: evaluation of kappa-agonists on cocaine pharmacodynamics and cocaine self-administration in humans. Journal of Pharmacology and Experimental Therapeutics 299: 147-158.

Walsh, S.L., Strain, E.C., Abreu, M.E., and Bigelow, G.E. 2001b. Enadoline, a selective kappa opioid agonist: comparison with butorphanol and hydromorphone in humans. Psychopharmacology 157: 151-162.

Wang, C.-I.A., and Lewis, R.J. 2009. Emerging structure-function relationships defining monoamine NSS transporter substrate and ligand affinity. Biochemical Pharmacology 79: 1083-1091.

Wang, H.-W., Li, C.-Z., Yang, Z.-F., Zheng, Y.-Q., Zhang, Y., and Liu, Y.-M. 2006. Electrophysiological effect of fluoxetine on Xenopus oocytes heterologously expressing human serotonin transporter. Acta Pharmacologica Sinica 27: 289293.

Wang, Y., Palmer, M.R., Cline, E.J., and Gerhardt, G.A. 1997. Effects of ethanol on striatal dopamine overflow and clearance: an in vivo electrochemical study. Alcohol 14: 593-601.

Wang, Y., Tang, K., Inan, S., Siebert, D., Holzgrabe, U., Lee, D.Y.W., Huang, P., Li, J.G., Cowan, A., and Liu-Chen, L.Y. 2005. Comparison of pharmacological activities of three distinct kappa ligands (salvinorin A, TRK-820 and 3FLB) on kappa opioid receptors in vitro and their antipruritic and antinociceptive activities in vivo. Journal of Pharmacology and Experimental Therapeutics 312: 220-230.

Wang, Y.L., Chen, Y., Xu, W., Lee, D.Y.W., Ma, Z.Z., Rawls, S.M., Cowan, A., and Liu-Chen, L.Y. 2008. 2-methoxymethyl-salvinorin B is a potent kappa opioid 
receptor agonist with longer lasting action in vivo than salvinorin A. Journal of Pharmacology and Experimental Therapeutics 324: 1073-1083.

Wannemacher, K.M., Yadav, P.N., and Howells, R.D. 2007. A select set of opioid ligands induce up-regulation by promoting the maturation and stability of the rat kappa-opioid receptor in human embryonic kidney 293 cells. Journal of Pharmacology and Experimental Therapeutics 323: 614-625.

Wells, J.E., Baxter, J., and Schaff, D. 2007. Substance use disorders in Te Rau Hinengaro: the New Zealand mental health survey. Alcohol Advisory Council of New Zealand, Wellington.

Wesson, D.R., and Smith, D.E. 2010. Buprenorphine in the treatment of opiate dependence. Journal of Psychoactive Drugs 42: 161-175.

Wilkins, C., Girling, M., and Sweetsur, P. 2006. Recent trends in illegal drug use in New Zealand, 2006: findings from the combined modules of the 2006 illicit drug monitoring system (IDMS). Final report. Centre for social and health outcomes research and evaluation \& te ropu whariki, Massey University, Auckland.

Wilkins, C., Reilly, J., Rose, E., Roy, D., and Pledger, M. 2004. The socio-economic impact of amphetamine type stimulants in New Zealand. Final Report. Centre for social and helath outcomes research and evaluation. Massey University, Auckland.

Willins, D.L., and Meltzer, H.Y. 1998. Serotonin 5-HT $2 \mathrm{C}$ agonists selectively inhibit morphine-induced dopamine efflux in the nucleus accumbens. Brain Research 781: 291-299.

Willmore-Fordham, C.B., Krall, D.M., McCurdy, C.R., and Kinder, D.H. 2007. The hallucinogen derived from Salvia divinorum, salvinorin A, has kappa-opioid agonist discriminative stimulus effects in rats. Neuropharmacology 53: 481-486.

Wu, Q., Reith, M.E.A., Kuhar, M.J., Carroll, F.I., and Garris, P.A. 2001. Preferential increases in nucleus accumbens dopamine after systemic cocaine administration are caused by unique characteristics of dopamine neurotransmission. The Journal of Neuroscience 21: 6338-6347.

Xia, Y.-F., He, L., Whistler, J.L., and Hjelmstad, G.O. 2008. Acute amphetamine exposure selectively desensitizes kappa-opioid receptors in the nucleus accumbens. Neuropsychopharmacology 33: 892-900.

Xiao, Z.-W., Cao, C.-Y., Wang, Z.-X., Li, J.-X., Liao, H.-Y., and Zhang, X.-X. 2006. Changes of dopamine transporter function in striatum during acute morphine addiction and its abstinence in rhesus monkey. Chinese Medical Journal 119: 1802-1807.

Xie, Z., and Miller, G.M. 2009. A receptor mechanism for methamphetamine action in dopamine transporter regulation in brain. Journal of Pharmacology and Experimental Therapeutics 330: 316-325.

Xu, H., Wang, X., Partilla, J.S., Bishop-Mathis, K., Benaderet, T.S., Dersch, C.M., Simpson, D.S., Prisinzano, T.E., and Rothman, R.B. 2008. Differential effects of opioid agonists on $\mathrm{G}$ protein expression in $\mathrm{CHO}$ cells expressing cloned human opioid receptors. Brain Research Bulletin 77: 49-54.

Xu, W., Yoon, S.-I., Huang, P., Wang, Y., Chen, C., Chong, P.L.-G., and Liu-Chen, L.Y. 2006. Localization of the $\kappa$ opioid receptor in lipid rafts. The Journal of Pharmacology and Experimental Therapeutics 317: 1295-1306.

Yahyavi-Firouz-Abadi, N., and See, R.S. 2009. Anti-relapse medications: preclinical models for drug addiction treatment. Pharmacology \& Therapeutics 124: 235247.

Yamashita, A., Singh, S.K., Kawate, T., Jin, Y., and Gouaux, E. 2005. Crystal structure of a bacterial homologue of $\mathrm{Na}+\mathrm{Cl}$-dependent neurotransmitter transporters. Nature 437: 215-223. 
Yan, F., Mosier, P.D., Westkaemper, R.B., Stewart, J., Zjawiony, J.K., Vortherms, T.A., Sheffler, D.J., and Roth, B.L. 2005. Identification of the molecular mechanisms by which the diterpenoid salvinorin A binds to kappa-opioid receptors. Biochemistry 44: 8643-8651.

Yang, L., Wei, X., Chen, R., Liu-Chen, L.-Y., Ma, Z., and Lee, D.Y.W. 2009. Synthesis and biological evaluation of $\mathrm{C}-12$ triazole and oxadiazole analgos of salvionrin A. Bioorganic \& Medicinal Chemistry Letters 19: 1301-1304.

Yang, Y.-K., Yao, W.J., Yeh, T.L., Lee, I.H., Chen, P.S., Lu, R.B., and Chiu, N.T. 2008. Decreased dopamine transporter availability in male smokers - A dual isotope SPECT study. Progress in Neuro-Psychopharmacology \& Biological Psychiatry 32: 274-279.

Yasuda, K., Raynor, K., Kong, H., Breder, C.D., Takeda, J., Reisine, T., and Bell, G.I. 1993. Cloning and functional comparisono of $\kappa$ and $\delta$ opioid receptors from mouse brain. Proceedings of the National Academy of Sciences of the United States of America 90: 6736-6740.

Yim, H.J., and Gonzales, R.A. 2000. Ethanol-induced increases in dopamine extracellular concentration in rat nucleus accumbens are accounted for by increased release and not uptake inhibition. Alcohol 22: 107-115.

Yim, H.J., Schallert, T., Randall, P.K., and Gonzales, R.A. 1998. Comparison of local and systemic ethanol effects on extracellular dopamine concentration in rat nucleus accumbens by microdialysis. Alcoholism: Clinical and Experimental Research 22: 107-115.

Yoshimoto, K., Ueda, S., Nishi, M., Yang, Y.-K., Matsushita, H., Takeuchi, Y., Kato, B., Kawai, Y., Noritake, K., Kaneda, S., et al. 2000. Changes in dopamine transporter and c-Fos expression in the nucleus accumbens of alcohol-tolerant rats. Alcoholism: Clinical and Experimental Research 24: 361-365.

Zakharova, E., Collins, S.L., Aberg, M., Kumar, A., Fernandez, F., and Izenwasser, S. 2008a. Depletion of serotonin decreases the effects of the kappa-opioid receptor agonist U-69593 on cocaine-stimulated acitivity. European Journal of Pharmacology 586: 123-129.

Zakharova, E., Collins, S.L., Kumar, A., Fernandez, F., and Izenwasser, S. 2008b. Depletion of serotonin decreases the effects of the kappa-opioid receptor agonist U-69593 on cocaine-stimulated activity. European Journal of Pharmacology 586: $123-129$.

Zapata, A., Kivell, B., Han, Y., Javitch, J.A., Bolan, E.A., Kuraguntla, D., Jaligam, V., Oz, M., Jayanthi, L.D., Samuvel, D.J., et al. 2007. Regulation of dopamine transporter function and cell surface expression by D3 dopamine receptors. Journal of Biological Chemistry 282: 35842-35854.

Zapata, A., and Shippenberg, T.S. 2002. D-3 receptor ligands modulate extracellular dopamine clearance in the nucleus accumbens. Journal of Neurochemistry 81: 1035-1042.

Zapata, A., and Shippenberg, T.S. 2006. Endogenous kappa opioid receptor systems modulate the responsiveness of mesoaccumbal dopamine neurons to ethanol. Alcoholism-Clinical and Experimental Research 30: 592-597.

Zapata, A., Witkin, J.M., and Shippenberg, T.S. 2001. Selective D3 receptor agonist effects of (+)-PD128907 on dialysate dopamine at low doses. Neuropharmacology 41: 351-359.

Zhang, H., Shi, Y.G., Woods, J.H., Watson, S.J., and Ko, M.C. 2007. Central kappaopioid receptor-mediated antidepressant-like effects of nor-binaltorphimine: behavioral and BDNF mRNA expression studies. European Journal of Pharmacology 570: 89-96. 
Zhang, L., Elmer, L.W., and Little, K.Y. 1998. Expression and regulation of the human dopamine transporter in a neuronal cell line. Molecular Brain Research 59: 6673.

Zhang, Y., Butelman, E.R., Schlussman, S.D., Ho, A., and Kreek, M.J. 2004. Effect of the endogenous $\mathrm{k}$ opioid agonist dynorphin $\mathrm{A}(1-17)$ on cocaine-evoked increases in striatal dopamine levels and cocaine-induced place preference in C57BL/6J mice. Psychopharmacology 172: 422-429.

Zhang, Y., Butelman, E.R., Schlussman, S.D., Ho, A., and Kreek, M.J. 2005. Effects of the plant-derived hallucinogen salvinorin A on basal dopamine levels in the caudate putamen and in a conditioned place aversion assay in mice: agonist actions at kappa opioid receptors. Psychopharmacology 179: 551-558.

Zhao, P., Yang, L., Lopez, J.A., Fan, J., Burchfield, J.G., Bai, L., Hong, W., Xu, T., and James, D.E. 2009. Variations in the requirement for v-SNAREs in GLUT4 trafficking in adipocytes. Journal of Cell Science 122: 3472-3480.

Zhou, F.-M., Liang, Y., Salas, R., Zhang, L., De Biasi, M., and Dani, J.A. 2005. Corelease of dopamine and serotonin from striatal dopamine terminals. Neuron 46: $65-74$.

Zhu, C.-B., Carneiro, A.M., Dostmann, W.R., Hewlett, W.A., and Blakely, R.D. 2005. p38 MAPK activation elevates serotonin transport activity via a traffickingindependent, protein phosphatase 2A-dependent process. The Journal of Biological Chemistry 280: 15649-15658.

Zhu, J., Apparsundaram, S., and Dwoskin, L.P. 2009. Nicotinic receptor activation increases [H-3]dopamine uptake and cell surface expression of dopamine transporters in rat prefrontal cortex. Journal of Pharmacology and Experimental Therapeutics 328: 931-939. 